Old Dominion University

ODU Digital Commons

Summer 2003

\title{
Geographic Variation in the Morphology of Crotalus horridus (Serpentes: Viperidae)
}

John Robert Allsteadt

Old Dominion University

Follow this and additional works at: https://digitalcommons.odu.edu/biology_etds

Part of the Biostatistics Commons, Ecology and Evolutionary Biology Commons, and the Zoology Commons

\section{Recommended Citation}

Allsteadt, John R.. "Geographic Variation in the Morphology of Crotalus horridus (Serpentes: Viperidae)" (2003). Doctor of Philosophy (PhD), Dissertation, Biological Sciences, Old Dominion University, DOI: $10.25777 / 44 f 7-3 y 27$

https://digitalcommons.odu.edu/biology_etds/46

This Dissertation is brought to you for free and open access by the Biological Sciences at ODU Digital Commons. It has been accepted for inclusion in Biological Sciences Theses \& Dissertations by an authorized administrator of ODU Digital Commons. For more information, please contact digitalcommons@odu.edu. 


\title{
GEOGRAPHIC VARIATION IN THE MORPHOLOGY OF \\ CROTALUS HORRIDUS (SERPENTES: VIPERIDAE)
}

\author{
by \\ John Robert Allsteadt \\ M.S. August 1993, University of North Dakota \\ B.A. June 1987, Lawrence University \\ A Thesis Submitted to the Faculty of \\ Old Dominion University in Partial Fulfillment of the \\ Requirement for the Degree of \\ DOCTOR OF PHILOSOPHY \\ ECOLOGICAL SCIENCES \\ OLD DOMINION UNIVERSITY \\ August 2003
}

Approved by:

Alan H. Savitzley (Director)

CXthia M. Jones (XYlember)

Dayanand N. Naik (Mémber)

Robert K. Rose (Member) 


\author{
ABSTRACT \\ GEOGRAPHIC VARIATION IN THE MORPHOLOGY OF \\ CROTALUS HORRIDUS (SERPENTES: VIPERIDAE) \\ John Robert Allsteadt \\ Old Dominion University, 2003 \\ Director: Dr. Alan H. Savitzky
}

The Timber Rattlesnake (Crotalus horridus) occurs in discontinuous populations throughout the eastern and central United States. The species exhibits high levels of polymorphism in morphological traits, especially in coloration and pattern. Previous studies recognized either distinct northern and southern subspecies or three regional morphs (northern, southern, and western), but conflicting data sets and limited geographic sampling of previous studies have left the relationships among those regional variants unclear. In this study, univariate and multivariate statistics, together with a geographic information system, were used to analyze geographic variation in 36 morphological characters recorded from 2,420 specimens of $C$. horridus across its range.

Sexual dimorphism was strong in ventral and subcaudal scales, and weak to moderate in band length and band spacing. Univariate analyses detected substantial geographic variation in all meristic characters. Scutellation exhibited a general northsouth pattern of variation, and most scale counts averaged higher in southern regions. Pattern characters differentiated the northeastern, central-eastern, and north-central regions from the southern and western regions. Coloration displayed a pattern of strong clinal variation among three broad areas consisting of the combined northeastern, centraleastern, and southern Appalachian regions, the northwestern regions, and the southern 
regions. Morphometric characters exhibited a general north-south pattern of geographic variation, with larger head and body sizes in southern regions.

Principal component analysis indicated that band length was the most important variable for characterizing geographic variation. The northeastern regions remained moderately distinct in all multivariate cluster analyses. The northwestern regions appeared very distinct in most cluster analyses for females. However, the clusters in all models showed extensive geographic overlap. Percentage maps of clusters revealed two north-south patterns of clinal variation among the northeastern, northwestern, and southern regions. The discordant patterns of variation among individual characters, the overlapping patterns of coloration, and the extensive overlap among the multivariate clusters collectively indicate that zones of intergradation among subpopulations of $C$. horridus are much broader than previously thought. Furthermore, the general patterns of geographic variation are strongly clinal and support the conclusion that $C$. horridus is a single widespread, polymorphic species. 
This dissertation is dedicated to my mother, Barbara Allsteadt, for her unconditional love and support. 


\section{ACKNOWLEDGMENTS}

Many people have contributed to the final completion of this dissertation. I give special thanks to my academic advisor, Alan Savitzky, who was critically involved in every aspect of this research, constantly stimulated valuable discussion, and rigorously reviewed my progress, writing, and organization. I also thank Dayanand Naik for his extensive assistance with the statistical analysis. I am grateful to Cynthia Jones and Robert Rose for critically reviewing the manuscript and making valuable improvements to the final dissertation. I extend special thanks to Christopher Petersen for his extensive assistance with the geographic information system used in this study, which proved to be an integral part of my research.

I greatly appreciate having had such extensive access to major museum collections across the United States. I thank the following museum curators and collection managers for allowing me to examine specimens in their care, either while visiting their facilities or through the loan of specimens: Charles Myers, Darrel Frost, and Linda Ford (AMNH), Ted Daeschler, Leo Joseph, and Ned Gilmore (ANSP), Arthur Scott (APSU), Nancy McCartney, Mary Suter, and Bryan McDade (ARK), Craig Guyer (AUM), Ginger Clark (BEECS), Andrew Simons (BMNH), Mary Hennen and Steven Sullivan (CA), John Wiens, Ellen Censky, and Steve Rodgers (CM), Harry Greene, John Friel, and Charles Dardia (CU), Harold Voris and Alan Resetar (FMNH), Christopher Phillips and John Petzing (INHS), William Duellman, John Simmons, and Hugo Alamillo (KU), Jim McGuire and Frank Burbrink (LSUMZ), Jose Rosado (MCZ), Gary Casper (MPM), Edmund Zimmerer (MUSU), Alvin Braswell, William Palmer, and Jeffrey 
Beane (NCSM), Frank Pezold and Mark Antwine (NLU), Todd Hunsinger (NYSM), Alan Savitzky (ODU), Laurie Vitt (OMNH), Scott Moody and Willem Roosenburg (OUVC), Bradford Hollingsworth (SDSNH), Jeffrey Stewart (SIUC), Jim Dixon and Kathryn Vaughan (TCWC), John Ferner (TMC), Harold Dundee (TU), Wayne King, Max Nickerson, and David Auth (UF), Elizabeth McGhee (UGAMNH), Christopher Phillips and John Petzing (UIMNH), Perri Eason and Melinda LaManna (UL), Gregory Schneider (UMMZ), Patricia Freeman and Thomas Labedz (UNSM), Ronald Heyer, Robert Reynolds, and Steve Gotte (USNM), Jonathan Campbell (UTA), Carl Lieb (UTEP), Thomas Pauley (WVBS).

Partial funding for this project was provided by the American Museum of Natural History, the Carnegie Museum of Natural History, the Chicago Herpetological Society, the Field Museum of Natural History, the National Museum of Natural History, Old Dominion University, and Sigma Xi.

I thank Thomas Anton, Chad Cross, Konrad Mebert, Susan Rehorek, and Barbara Savitzky for many valuable discussions about herpetology. I also thank Hugo Alamillo, Todd Castoe, Ginger Clark, Peter and Rachel Clark, Jim Dixon, Eli Greenbaum, Ronald Heyer, Todd Hunsinger, Susan Rehorek, Jeffrey Stewart, and John Wiens for graciously opening their homes and providing a refuge while I was visiting museums. I thank a number of special people who helped me in many ways: Laura Duncan, Ken Farris, Robert Gatten, Deborah Hutchinson, Ju Long, John Morgan, Christopher and Dourina Petersen, Robert Powell, Robert Ratzlaff, Gabriel and Angela Rivera, Gail Stenberg, William Stevens, and Larry and Twila Wilson. I thank Peggy Lee for her enthusiasm and support. I must give special recognition to five educators who have made a great impact 
magnificent educators who have made a great impact on my life, prepared me for graduate-level studies, and motivated me with their passion for the biological sciences: Michael LaMarca, Jeffrey Lang, Michael O'Hare, Bradford Rence, Alan Savitzky, and Christopher Vaughan.

I thank Mark and Elizabeth Allsteadt for all of their interest and support for so many years, I never could have completed this study without their help. I especially thank my parents, Wayne and Barbara Allsteadt, for always helping me to pursue my interest in herpetology and for supporting my education in many ways. It was generous of them to permit me to study and house so many amphibians and reptiles, primarily snakes, in our home during so many years of discovery. Finally, my deepest thanks to Deepti Kudyadi for all of her assistance, understanding, and support. 


\section{TABLE OF CONTENTS}

Page

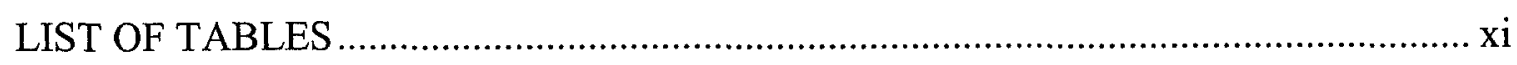

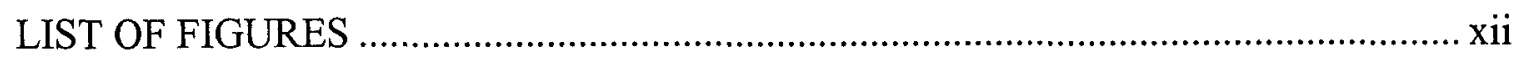

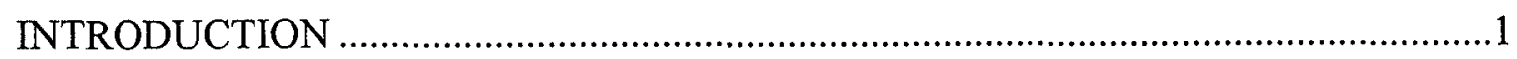

ZOOGEOGRAPHY OF THE CROTALINAE ...............................................5

RECENT DISTRIBUTION ..................................................................

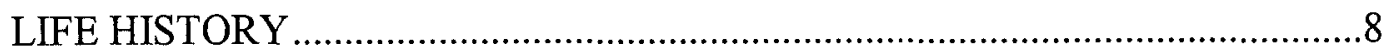

MATERIALS AND METHODS ..................................................................

STUDY SAMPLE AND CHARACTER SET ….........................................10

REGIONAL CLASSIFICATION USED FOR GEOGRAPHIC ANALYSIS ......13

UNIVARIATE ANALYSIS OF MERISTIC DATA .....................................16

GEOGRAPHIC ANALYSIS OF MERISTIC AND COLORATION DATA ......17

REGRESSION ANALYSIS OF MERISTIC DATA …....................................19

PRINCIPAL COMPONENT ANALYSIS OF MERISTIC DATA .....................20

CLUSTER ANALYSIS OF MERISTIC DATA ...............................................20

DISCRIMINANT FUNCTION ANALYSIS OF MERISTIC DATA...................23

GEOGRAPHIC ANALYSIS OF MULTIVARIATE CLUSTERS.....................24

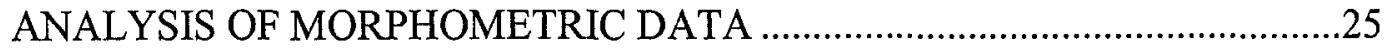

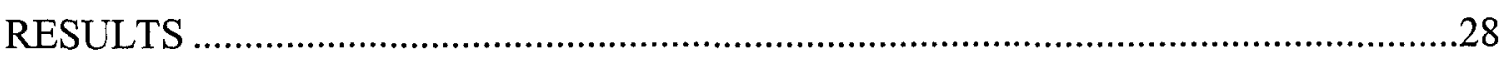

UNIVARIATE ANALYSIS OF MERISTIC DATA ......................................28

GEOGRAPHIC ANALYSIS OF MERISTIC DATA ....................................29

GEOGRAPHIC ANALYSIS OF COLORATION ............................................39

REGRESSION ANALYSIS OF MERISTIC DATA …...................................46

PRINCIPAL COMPONENT ANALYSIS OF MERISTIC DATA .....................51

CLUSTER ANALYSIS OF MERISTIC DATA ..............................................53

DISCRIMINANT FUNCTION ANALYSIS OF MERISTIC DATA..................55

GEOGRAPHIC ANALYSIS OF MULTIVARIATE CLUSTERS......................58

ANALYSIS OF MORPHOMETRIC DATA …..............................................69

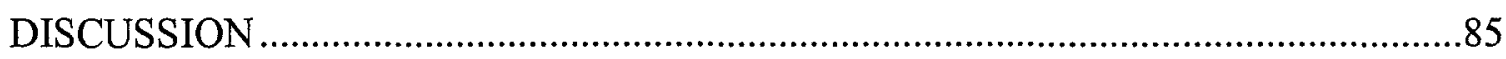

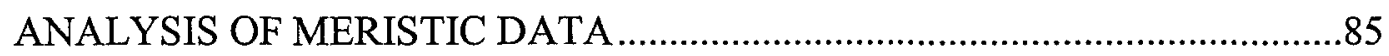

ANALYSIS OF COLORATION................................................................95

MULTIVARIATE ANALYSIS OF MERISTIC DATA …..............................97

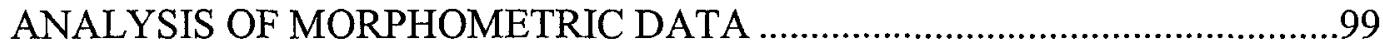




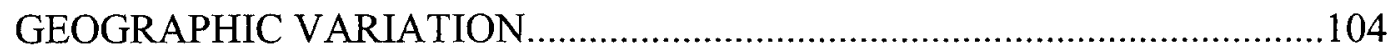

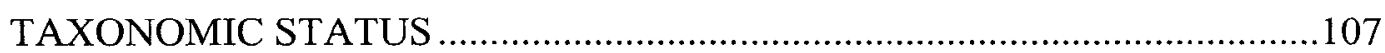

HISTORICAL ZOOGEOGRAPHY .............................................................113

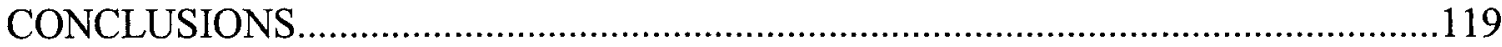

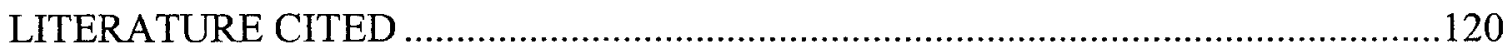

APPENDICES

APPENDIX I: SPECIMENS EXAMINED .....................................................134

APPENDIX II: MORPHOLOGICAL CHARACTERS .....................................146

APPENDIX III: REGIONAL CLASSIFICATION USED IN THIS STUDY ....151

APPENDIX IV: UNIVARIATE STATISTICS OF MERISTIC DATA FOR

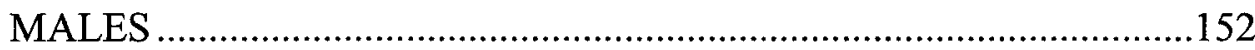

APPENDIX V: UNIVARIATE STATISTICS OF MERISTIC DATA FOR

FEMALES …….....................................................................166

APPENDIX VI: UNIVARIATE STATISTICS OF MERISTIC DATA FOR

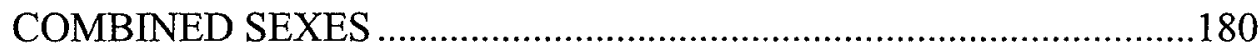

APPENDIX VII: PRINCIPAL COMPONENT ANALYSIS..............................194

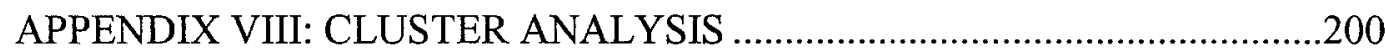

APPENDIX IX: CANONICAL DISCRIMINANT ANALYSIS ........................203

APPENDIX X: DISCRIMINANT FUNCTION ANALYSIS..............................206

APPENDIX XI: UNIVARIATE STATISTICS OF MOPHOMETRIC DATA

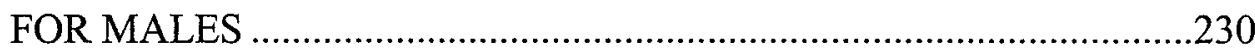

APPENDIX XII: UNIVARIATE STATISTICS OF MOPHOMETRIC DATA

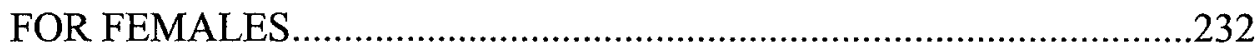

APPENDIX XIII: FACTOR ANALYSIS OF MORPHOMETRIC DATA........234

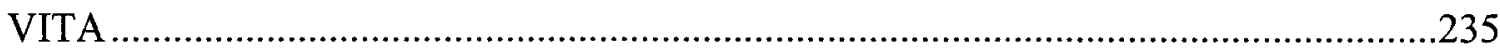




\section{LIST OF TABLES}

Table

Page

1. ANCOVA of six meristic relationships to test for the effects of sex using the entire data set

2. ANCOVA of ten morphometric relationships to test for the effects of sex using the entire data set.

3. ANCOVA of ten morphometric relationships to test for the effects of sex using data from the 12 selected study areas (Fig. 5)

4. ANCOVA of ten morphometric relationships to test for the effects of region using data from the 12 selected study areas (Fig. 5) 


\section{LIST OF FIGURES}

Figure

1. Map of the eastern United States showing the total sample sizes for combined sexes

2. Map of the eastern United States showing the total sample sizes for (A) males and (B) females

3. Regional classification used in this study .

4. Map showing the percentages of males and

females in samples from the 32 regions

5. The 12 selected study areas used for several analyses

6. Geographic variation in number of dorsal scale

rows in 32 regions

7. Geographic variation in ventral and subcaudal scales in 32 regions

8. Geographic variation in number of labial scales

in 32 regions.

9. Geographic variation in dorsal pattern elements in 32 regions.

10. Geographic variation of band length and band

spacing in 32 regions

11. Geographic variation in number of irregular pattern elements in 32 regions

12. Geographic variation in melanin density

13. Geographic variation in dorsal ground color

14. Geographic variation in dorsal head color

15. Geographic variation in postocular stripe of specimens $>900 \mathrm{~mm}$ SVI 
16. Geographic variation in coloration in 32 regions,

by frequency.

17. Regressions of dorsal pattern elements on band

length.

18. Regressions of dorsal pattern elements on band spacing and transverse elements

19. Regressions of transverse elements and subcaudal scales on ventral scales 50

20. Dendrograms for cluster analyses using Ward's

minimum-variance method.

21. Plot of first two canonical variables of three-cluster

models for combined sexes. 56

22. Plots of first two canonical variables of four-cluster and five-cluster models for combined sexes

23. Maps of geographic clusters of the two-cluster model for males.

24. Maps of geographic clusters of the two-cluster model for females

25. Maps of geographic clusters of the two-cluster model for combined sexes

26. Maps of geographic clusters as percentages of regional populations for the two-cluster models

27. Maps of geographic clusters of the three-cluster model for males.

28. Maps of geographic clusters of the three-cluster model for females

29. Maps of geographic clusters of the three-cluster model for combined sexes

30. Maps of geographic clusters as percentages of regional populations for the three-cluster models.

31. Regression of tail length on snout-vent length 
32. Regression of head lengths on snout-vent length .......................................... 73

33. Regression of quadrate and mandible lengths on

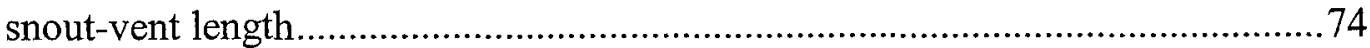

34. Regression of head length at the supraoccipital

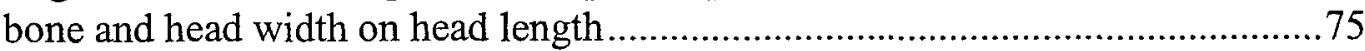

35. Regression of quadrate and mandible lengths on head length at the mandible 76

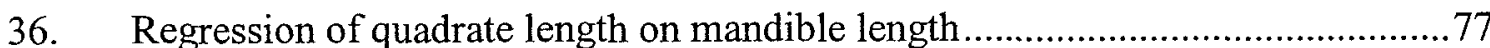




\section{INTRODUCTION}

Pitvipers (Crotalinae) that have widespread geographic distributions in the New World typically display large amounts of morphological variation, sometimes associated with different environments (Campbell and Lamar, 1989; Ernst, 1992; Greene, 1997). In many species, a major component of this variation is geographic. Different selective regimes acting in different habitats may produce significant geographic differentiation within a species (Cracraft, 1989; Endler, 1977; Gould and Johnston, 1972; Thorpe et al., 1995). This phenomenon is a major driving force in the process of speciation. Most species are thought to have evolved under conditions of geographic isolation (Mayr 1942), and indeed vicariant events may greatly affect geographic variation within a species (Thorpe et al., 1991, 1994). However, barriers to gene flow need not exist for spatial differentiation to occur in continental species (Endler, 1977; Gould and Johnston, 1972). The first step in a study of geographic variation is to describe spatial patterns of differentitation (Cracraft, 1989). Studies of geographic variation in morphology can illuminate the ecology, systematics, and phylogenetic history of a species.

Pitvipers invaded North and South America from Asia during the Tertiary, adapting to a variety of climates and habitats, and evolving into many species displaying a wide range of geographic variation both within and among species (Cadle, 1987; Campbell and Lamar, 1989; Ernst, 1992; Parkinson et al., 2002; Wüster et al., 2002).

The model journal for this dissertation is Herpetological Monographs. 
Extensive geographic variation in morphology has been described in various taxa, including Agkistrodon contortrix (Gloyd and Conant, 1990), Bothriechis (Crother et al., 1992), the Bothrops complex (Gutberlet and Harvey, 2002), the Crotalus atrox complex (Gloyd, 1940; Grismer et al., 1994; Klauber, 1956, 1972; Murphy et al., 1995), the Crotalus durissus complex (Campbell and Lamar, 1989), the Crotalus viridis complex (Klauber, 1956, 1972), and Lachesis (Campbell and Lamar, 1989). Similarly, molecular studies have detected extensive geographic variation within Agkistrodon (Knight et al., 1992; Parkinson et al., 2000), Bothriechis (Crother et al., 1992), the Bothrops complex (Parkinson et al., 2002; Wüster et al., 2002), the Crotalus atrox complex (Murphy et al., 1995; Murphy et al., 2002), the Crotalus durissus complex (Murphy et al., 2002; Wüster et al., 2002), the Crotalus viridis complex (Pook et al., 2000; Douglas et al., 2002), and Lachesis (Zamudio and Greene, 1997). Here, I examine the geographic variation within another wide-ranging pitviper, Crotalus horridus.

Crotalus horridus, the Timber Rattlesnake, occurs in discontinuous populations throughout the eastern and central United States. The species exhibits high levels of polymorphism in morphological traits, including coloration, pattern, and scutellation, and as many as three regional morphs have been recognized across its range (Martin, 1992a). There have been only a few studies of morphological variation within Crotalus horridus. Gloyd $(1935,1940)$ placed C. horridus in the C. durissus group and distinguished between a northern form, the Timber Rattlesnake proper (C. h. horridus Latreille, 1801) and a southern form, the Canebrake Rattlesnake (C. h. atricaudatus Linnaeus, 1758). The Canebrake was distinguished from the nominate subspecies on the basis of higher numbers of dorsal scale rows, ventral scales, and subcaudal scales, a well-defined 
postocular stripe, and larger body size. In contrast, Pisani et al. (1973) found such extensive variation across the species' range that they could not recognize distinct subspecies. For example, western populations exhibit characteristics of both the northeastern and southern morphs. However, some variables reportedly characteristic of southern variants were not considered in their analysis (e.g., middorsal stripe, ground color, and postocular stripe), which was limited to a subset of Gloyd's original data. In contrast, Brown and Ernst (1986) found that a combination of adult size, pattern, number of dorsal scale rows, and number of ventral scales discriminated between the two subspecies described by Gloyd. However, their study, based on a relatively small sample, was limited to a restricted region of sharp clinal variation in the eastern United States. Martin (1992a) sought to reconcile the conflicts among earlier studies by recognizing three regional morphs, including one from the upper Midwest. Among those three regional morphs, four color morphs have been recognized: (1) yellow northeastern morph, (2) black northeastern morph, (3) southern morph, and (4) western morph (Brown, 1993; Conant and Collins, 1991; Martin, 1992a). Additional forms are classified as intermediates and reflect the high levels of variation that occur among specimens from the three regions. Clark et al. (2003) described three mtDNA lineages corresponding to separate eastern and western populations; their results did not correspond to Gloyd's subspecies $(1935,1940)$ or to Martin's regional morphs (1992a). Thus, the taxonomic status of populations of $C$. horridus remains unclear due to incomplete and conflicting data sets, as well as the limited geographic sampling of previous morphological studies. A more comprehensive study of Crotalus horridus is required to understand the complex patterns of geographic variation within this wide-ranging species. Rather than 
simply testing the previously defined morphs and examining a limited number of specimens, I examined variation among populations using multivariate statistics applied to a large morphological data set. That procedure better characterized geographic variation within the species. A geographic information system (GIS) was used to display the results of the statistical analyses and to view broad geographic patterns. The results of that analysis form the basis for a discussion of the evolutionary history of Crotalus horridus and the taxonomic status of its populations. This study had the following objectives:

(1) To examine geographic variation in individual morphological characters of Crotalus horridus.

(2) To determine the extent of sexual dimorphism within C. horridus.

(3) To determine whether distinct geographic units exist within C. horridus.

(4) To explore the relationship between variation in morphological characters of C. horridus and environmental variables.

(5) To interpret morphological variation within C. horridus with regard to the evolutionary history of the species and the taxonomic status of its populations. 


\section{Zoogeography of the Crotalinae}

The center of origin of the viperid subfamily Crotalinae is southern Asia (Brattstrom, 1964; Cadle, 1987; Darlington, 1957; Gloyd and Conant, 1990; Kraus et al., 1996; Marx and Rabb, 1965; Rage, 1987). The arrival date of the Crotalinae in North America has not been established conclusively. Brattstrom (1964) postulated that the Crotalinae crossed the Bering Land Bridge into North America in the Paleocene and Eocene. However, the oldest viperid fossils discovered in North America date from the early Miocene of Nebraska and the late Miocene of Texas and Nebraska (Holman, 1977, $1979,1981)$. Thus, in the most conservative view this dispersal occurred by the late Oligocene or early Miocene (Conant, 1990; Holman, 1981; Van Devender and Conant, 1990). Some molecular evidence indicates that crotalines colonized the New World even later, in the mid-Pliocene (Knight et al., 1992). However, Crother et al. (1992) used geological data to reason that the ancestor of Bothriechis was already in Middle America between the late Cretaceous and early Eocene.

Some researchers have hypothesized that the New World Crotalinae are polyphyletic, reflecting multiple dispersal events into the New World (Brattstrom, 1964; Burger, 1971; Gloyd and Conant, 1990). However, recent molecular data indicate that New World Crotalinae form a monophyletic group, suggesting a single migration across the Bering Land Bridge (Kraus et al., 1996; Parkinson, 1999; Parkinson et al., 2002). In any event, crotalines were established in North America by the early Miocene and probably radiated during the middle Miocene in response to climatic changes, habitat differentiation, and subsequent vicariant events (Gloyd and Conant, 1990; Van Devender 
and Conant, 1990). Fossil specimens suggest that most crotalines attained their present distributions by the middle or late Pliocene (Brattstrom, 1954, 1964). Molecular data indicate that a basal divergence within the New World Crotalinae resulted in a North American group, consisting of Agkistrodon, Crotalus, and Sistrurus, and a Neotropical group, consisting of Lachesis and the bothropoids (Gutberlet and Harvey, 2002;

Parkinson, 1999; Parkinson et al., 2002).

The modern rattlesnakes originated in the mid-Cenozoic and are placed in two genera (Crotalus and Sistrurus), which may have diverged from each other as early as 30 Ma (Knight et al., 1993). Brattstrom (1954, 1958, 1964, 1967) suggested that most species of rattlesnakes had evolved and dispersed into their present ranges by the middle or late Pliocene based on the fossil record. Molecular data indicate that differentiation within the Crotalus viridis complex dates back to the Pliocene and continued into the Pleistocene (Douglas et al., 2002). Pook et al. (2000) estimated that eastern and western lineages of $C$. viridis split earlier, in the middle Miocene to early Pliocene. The exact center of origin and dispersal of the rattlesnakes is unknown, due to their limited fossil record. However, the present pattern of distribution and species richness indicates a center of origin on the Mexican Plateau (Campbell and Lamar, 1989; Ernst, 1992).

The ancestor of Crotalus horridus probably dispersed from southwestern North America into more temperate habitats of eastern North America between the early Miocene and late Pliocene. Fossils of $C$. horridus are widely represented throughout eastern North America in the Pleistocene (Holman, 1995). Populations of C. horridus must have undergone complex changes in distribution due to the cyclic glaciations of the Pleistocene (Martin, 1992a). The zoogeography of C. horridus is especially compelling 
because it has been placed within the C. durissus group (Brattstrom, 1964; Foote and MacMahon, 1977; Gloyd, 1940), which consists largely of species that inhabit rocky, arid habitats in southwestern North America, Central America, and South America. In contrast, $C$. horridus occupies the cooler and moister habitats of temperate forests of eastern North America and occurs at relatively high latitudes and elevations. Recent molecular evidence places $C$. horridus within a C. viridis + C. scutulatus group (Murphy et al., 2002), which includes the wide-ranging C. viridis complex (Douglas et al., 2002; Pook et al., 2000), arguably the geographic counterpart to C. horridus in western North America. However, molecular analyses of the relationship of $C$. horridus to the $C$. viridis group are inconsistent, C. horridus and C. willardi are resolved as sister taxa in a tree based on combined RNA gene data (Murphy et al., 2002). Thus, the taxonomic position of C. horridus within the genus and the taxonomic status of its constituent populations remain unclear.

\section{Recent Distribution of Crotalus horridus}

The most comprehensive distribution map of Crotalus horridus was compiled by Martin (1992a). In his analysis an eastern morph occurs from northeastern Georgia throughout the Appalachian Mountains and into New England. The western limits of the eastern morph are sharply defined in southern Ohio, eastern Kentucky, and east-central Tennessee. A southern morph occurs on the Coastal Plain from eastern Texas throughout the Gulf states to northeastern Florida, thence north through the Carolinas into extreme southeastern Virginia. The southern form also extends northward within the Mississippi 
River drainage to southeastern Missouri and extreme southern Illinois. A western morph is distributed from eastern Oklahoma, northwestern Arkansas, and throughout Missouri and extends northward along the Mississippi River from southern Illinois to southeastern Minnesota and southwestern Wisconsin. The western morph also extends from southern Illinois and southern Indiana south to western and central Kentucky, central Tennessee, and northeastern Alabama. C. horridus appears to tolerate a wide range of elevations, from sea level to $2,000 \mathrm{~m}$, and the highest elevations of hibernacula range from $400 \mathrm{~m}$ in New York to $1400 \mathrm{~m}$ in the southern Appalachians (Martin, 1992a).

\section{Life History}

Several long-term studies have quantified essential life history traits of Crotalus horridus (Brown, 1991; Martin, 1988; Martin, 1992ab), primarily in the northern parts of its range. Timber Rattlesnakes are late-maturing viperids characterized by viviparity, high survivorship after the first year of life, and relatively substantial longevity (Martin, 1992a; Parker and Plummer, 1987). Maximum longevity is estimated to be 25-50 years, and age at first reproduction is 4-11 years (Brown, 1991, 1993; Fitch, 1985; Galligan and Dunson, 1979; Gibbons, 1972; Keenlyne, 1978; Martin, 1988). However, C. horridus also exhibits low fecundity and low reproductive frequency. Females reproduce every 27 years depending on latitude, annual variation in climate, and amount of food ingested (Brown, 1991; Martin, 1993). The activity season is inversely related to latitude, and northern climates profoundly affect annual growth and adult body size. The minimum snout-vent length at maturity is roughly $900-1000 \mathrm{~mm}$ in the south and $700-850 \mathrm{~mm}$ in 
the north (Aldridge and Brown, 1995; Brown, 1991; Galligan and Dunson, 1979;

Gibbons, 1972; Keenlyne, 1978; Martin, 1988, 2002).

The density of integumentary melanin may be a thermal adaptation to cooler habitats, with darker individuals tending to occur in more densely forested and colder areas (Martin, 1992a). Thus, higher frequencies of melanism should be expected to occur at higher latitudes and elevations. However, melanistic Timber Rattlesnakes only occur in the mountains of the northeastern United States (Ernst, 1989). The lack of melanism in populations throughout Wisconsin and Minnesota is contrary to Martin's observations, although it may reflect the independent phylogenetic history of $C$. horridus at the northeastern and northwestern extremes of the species' range (Clark et al., 2003).

The number of dorsal scale rows varies among populations of Crotalus horridus (Gloyd, 1940). That feature may be directly related to girth and may be associated with prey size (Pough and Groves, 1983; but see Shine, 2002). Smaller mammals may be consumed more frequently by $C$. horridus at higher latitudes and elevations, whereas populations at lower elevations may rely more on larger prey. Warmer ambient temperatures may promote more rapid rates of digestion, and thus higher growth rates. 


\section{MATERIALS AND METHODS}

\section{Study Sample and Character Set}

I examined 2,420 preserved specimens of Crotalus horridus from 38 collections in the United States (Appendix I). The sample included specimens from 509 counties in 30 states and represented localities across the entire range of the species (Fig. 1). The sampling density was very uneven, with many specimens concentrated in relatively few areas of dense sampling, often in the vicinity of major research institutions. The sample included 1,274 males, 1,136 females, and 10 specimens of undetermined sex. The total sample included 1,974 independent specimens plus 446 specimens belonging to 58 broods of neonates. The independent specimens consisted of 1,054 males, 911 females, and 9 specimens of undetermined sex (Fig. 2). Multivariate statistics were performed on 1,626 independent specimens with no missing values; these consisted of 874 males, 751 females, and 1 specimen of undetermined sex. Specimens from 58 broods of neonates included 220 males, 225 females, and 1 specimen of undetermined sex.

Thirty-six meristic, morphometric, and coloration characters were recorded for each specimen of Crotalus horridus. Descriptions of all characters and their

abbreviations are provided in Appendix II. All head measurements were made using dial calipers. Statistical analyses were performed using SAS 8.01 (SAS, 2000), following version 6 of the SAS User's Guide (SAS, 1989). I examined patterns of geographic variation by analyzing individual meristic and coloration characters, performing a multivariate analysis of the meristic data, using a geographic information system to 


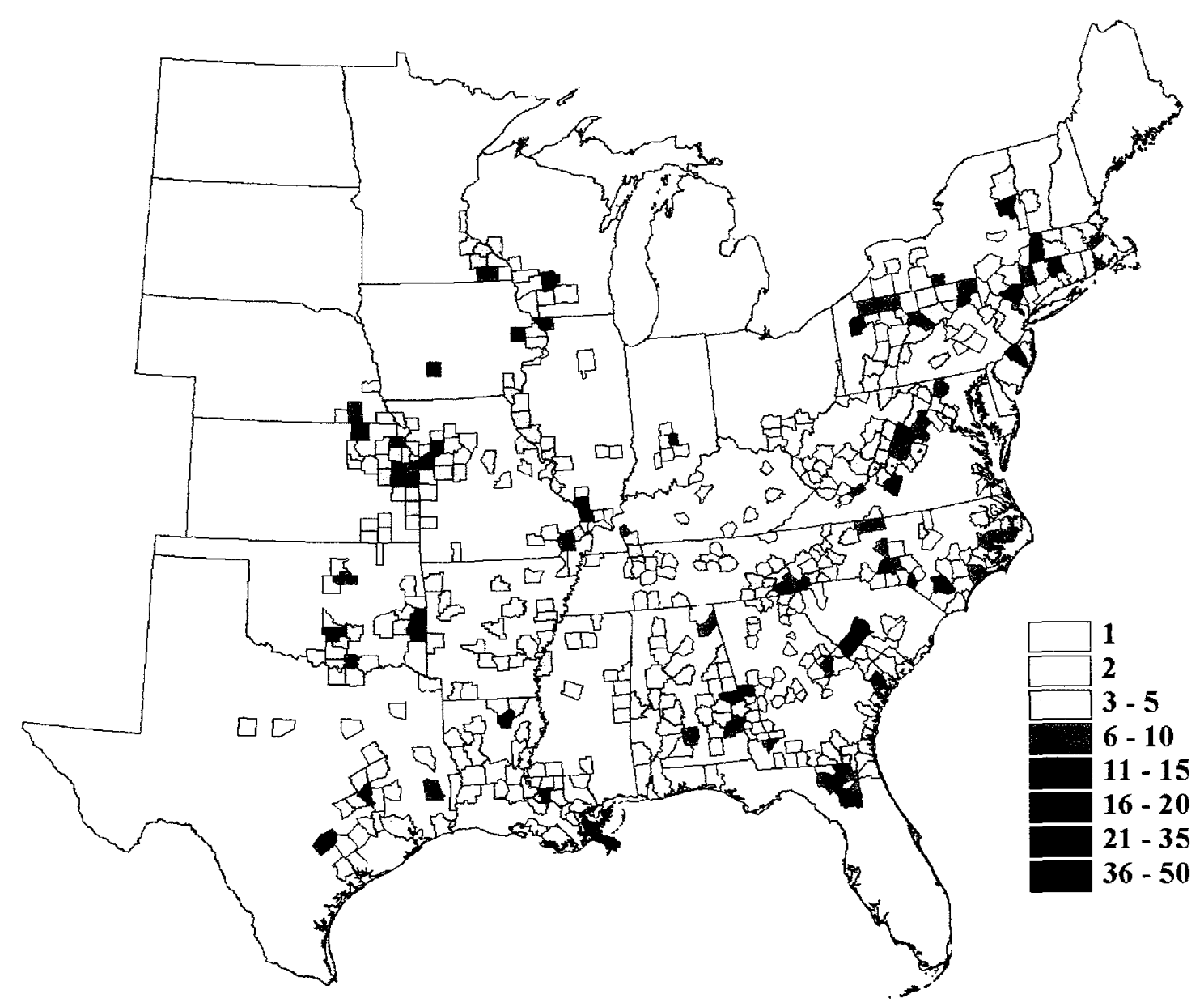

Fig. 1.-Map of the eastern United States showing the total sample sizes for combined sexes. The numbers in the key indicate the number of specimens examined from each county. 

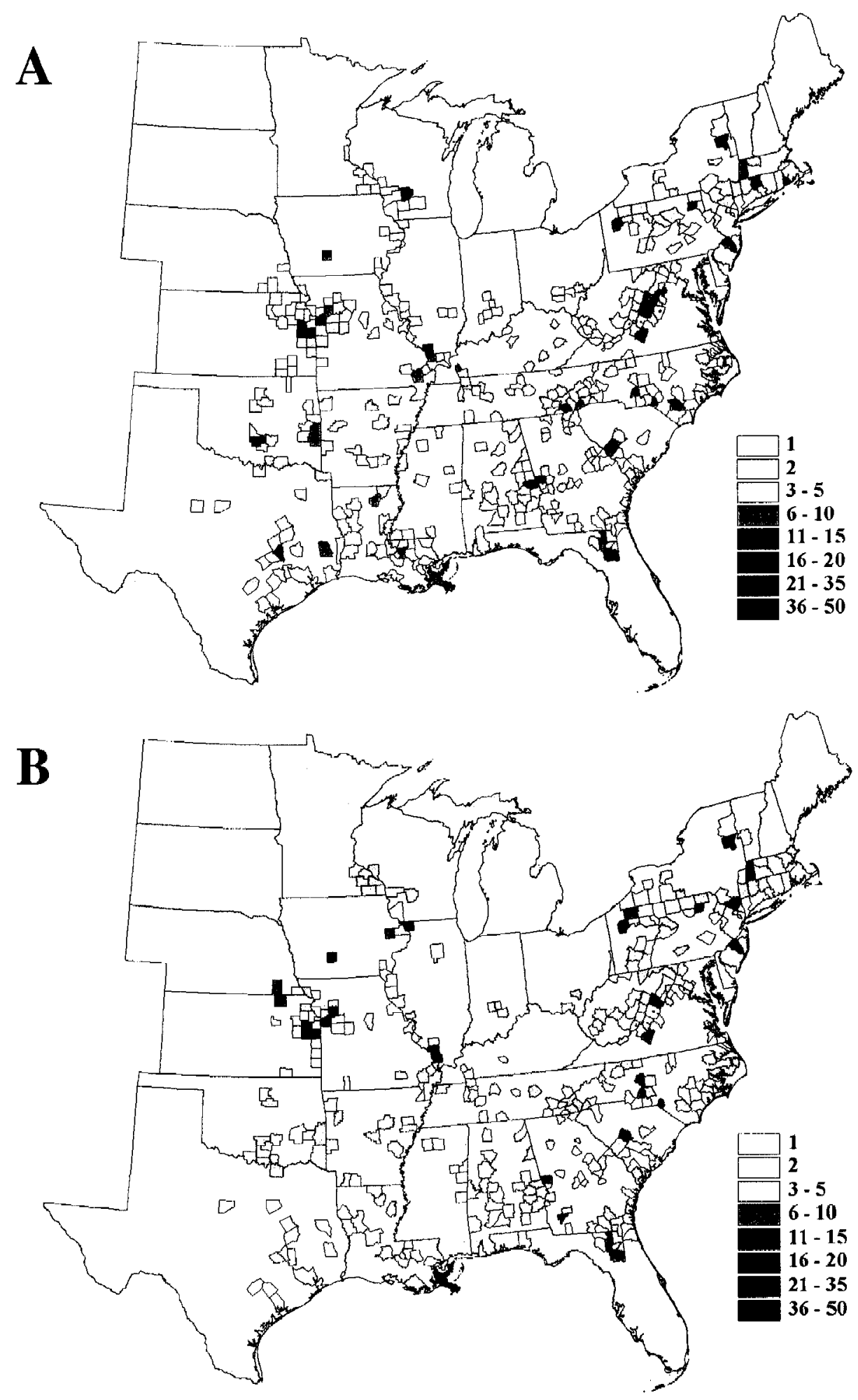

Fig. 2.- Map of the eastern United States showing the total sample sizes for (A) males and $(B)$ females. The numbers in the key indicate the number of specimens examined from each county. 
explore patterns within the univariate and multivariate data, and performing an analysis of covariance on the morphometric data.

\section{Regional Classification Used for Geographic Analysis}

The study area was divided into 32 regions based on regional sample size, geography, physiography, and general habitat type (Fig. 3). Descriptions of the regions and their abbreviations are provided in Appendix III. The percentages of males and females were equivalent in the northeastern regions; males dominated the southern samples, and females dominated the northwestern and central-western samples (Fig. 4). The regional classification was designed to consolidate areas of dense sampling into regions of homogeneous macrohabitat and to account for the uneven sampling densities across regions. I designated a relatively large number of regions to ensure that the analysis of individual characters was conservative and to avoid bias in favor of previous geographic classifications, such as Gloyd's $(1935,1940)$ subspecies or Martin's (1992a) regional morphs. Using a smaller number of larger regions, and especially the same regions as previous studies, would have introduced greater bias by limiting a priori the number of possible regional groupings.

Eight generic terms are used to describe general areas throughout the range of Crotalus horridus: northeastern, central-eastern, southeastern, north-central, southcentral, northwestern, central-western, and southwestern. These genearal areas are defined as follows in terms of the 32 primary regions recognized in this study: the northeastern regions consist of CTMA, NJCP, NYE, NYN, NYPA, and PAE. The 

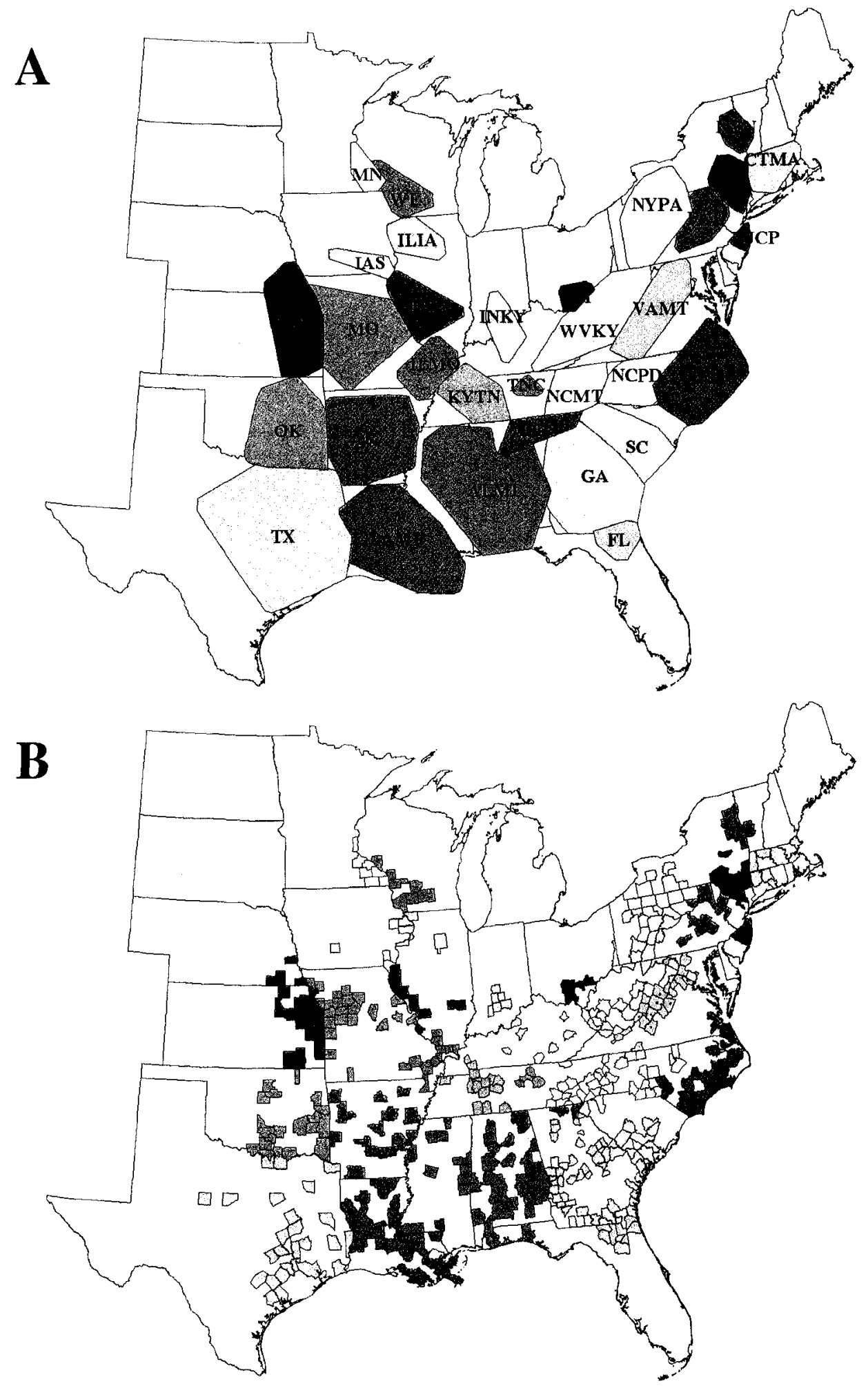

Fig. 3.-Regional classification used in this study. (A) Map of 32 geographic regions used in this study. Shapes of regions represent minimum convex polygons connecting outlying counties and do not represent ranges of populations. Descriptions and names of regions are provided in Appendix III. (B) Map of regions showing counties from which specimens were examined. 


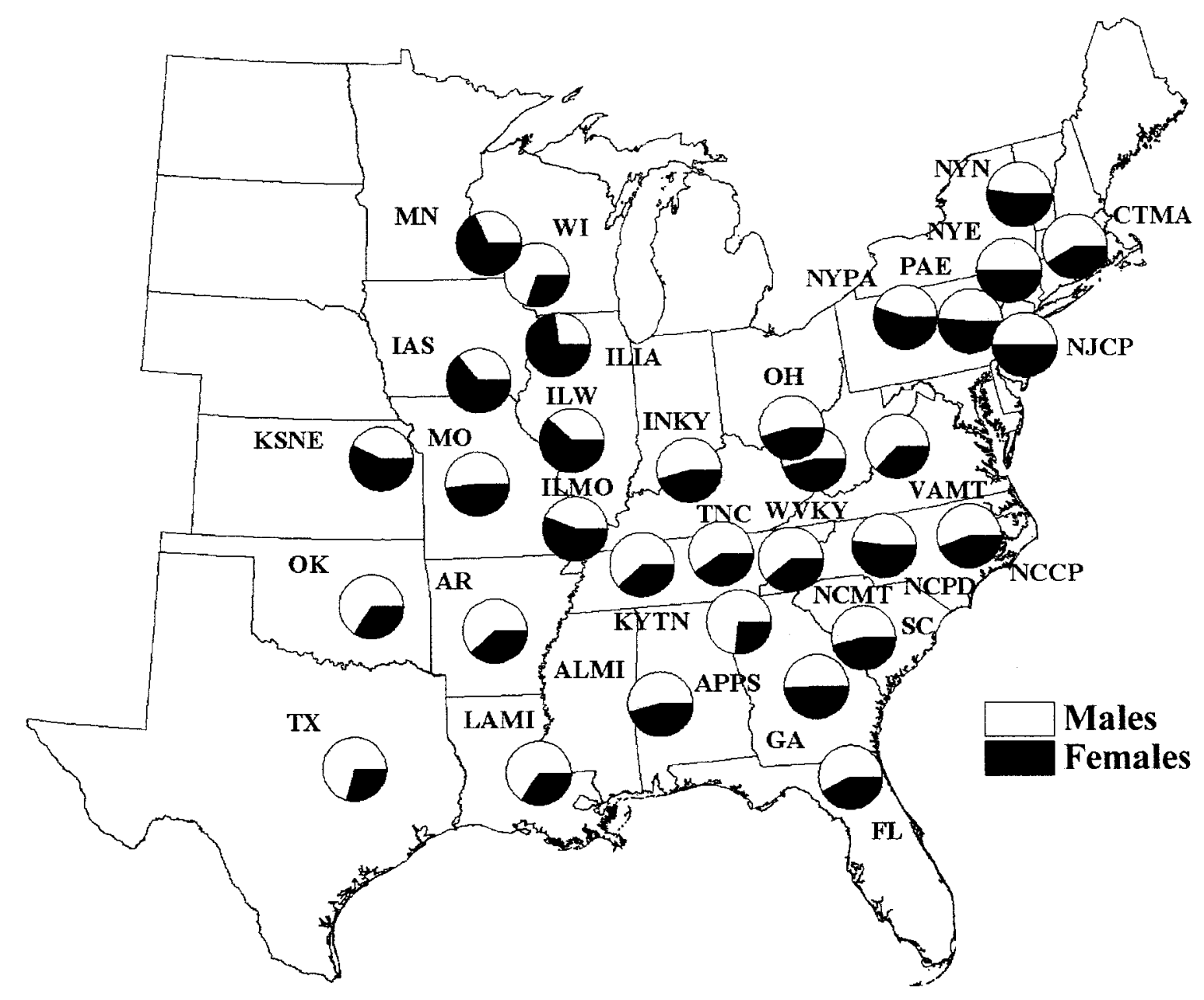

Fig. 4.-Map showing the percentages of males and females in samples from the 32 regions. Names and descriptions of regions are provided in Appendix III. 
central-eastern regions are defined by KYTN, NCMT, TNC, VAMT, and WVKY. The southeastern regions include FL, GA, NCCP, NCPD, and SC. The north-central regions consist of INKY, KYTN, OH, and TNC. The south-central regions are defined by ALMI and APPS. The northwestern regions consist of ILIA, MN, and WI. The central-western regions are defined by IAS, ILMO, ILW, KSNE, and MO. The southwestern regions include $\mathrm{AR}, \mathrm{LAMI}, \mathrm{OK}$, and TX.

The regional classification I used requires further explanation. The smallest geographic unit of the study was the county. Although some counties may contain strongly different habitats, most counties can be considered roughly homogeneous in habitat on the continental scale of this study. Because the morphological variants of Crotalus horridus have such large distributions, pooling counties into the 32 regions used here should not cause significant bias. The arbitrary divisions between IAS and ILW, MO and ILW, WVKY and INKY, and APPS and NCMT (see Appendix III for key to regional abbreviations) were established largely to accommodate small sample sizes in certain counties and to contend with the uneven geographic distribution of samples; those divisions should not affect the analysis significantly.

\section{Univariate Analysis of Meristic Data}

Means and descriptive statistics for 24 meristic characters of scutellation and pattern were computed in the 32 regions separately for males, females, and combined sexes, using the SAS MEANS procedure. The SAS UNIVARIATE procedure was performed to test for excessive skewness, kurtosis, and nonnormality. Logarithmic 
transformation was applied to the data, and the Fmax-test was used on males, females, and combined sexes in each region to test for homogeneity of variances. Analysis of variance was performed using the SAS ANOVA procedure to determine whether differences among regions were significantly different. A two-sample $t$-test was performed using the SAS TTEST procedure to detect significant sexual dimorphism in each meristic character. Separate $t$-tests were conducted for each meristic character using both the entire data set and 12 selected study areas. The 12 study areas were chosen from across the geographic range of the species and represented areas where large numbers of specimens were concentrated in relatively small homogeneous regions (Fig. 5).

\section{Geographic Analysis of Meristic and Coloration Data}

Spatial analysis of scutellation and pattern was performed for males, females, and combined sexes using a geographic information system (GIS; ArcView, version 3.2, Environmental Systems Research Institute (ESRI), 1992-1999). The mean value of each meristic character was calculated by region using the untransformed data. The regional means were joined to the GIS using the regional abbreviations. Each regional mean was classified into one of four levels using even intervals and then mapped as a group of contiguous counties on a county base map of the United States (ArcUSA 1:2M data set; ESRI, 1997) to examine geographic patterns in the data. On each map, the color of a county represents the regional mean for the entire region to which that county belongs, not the mean for the individual county. 

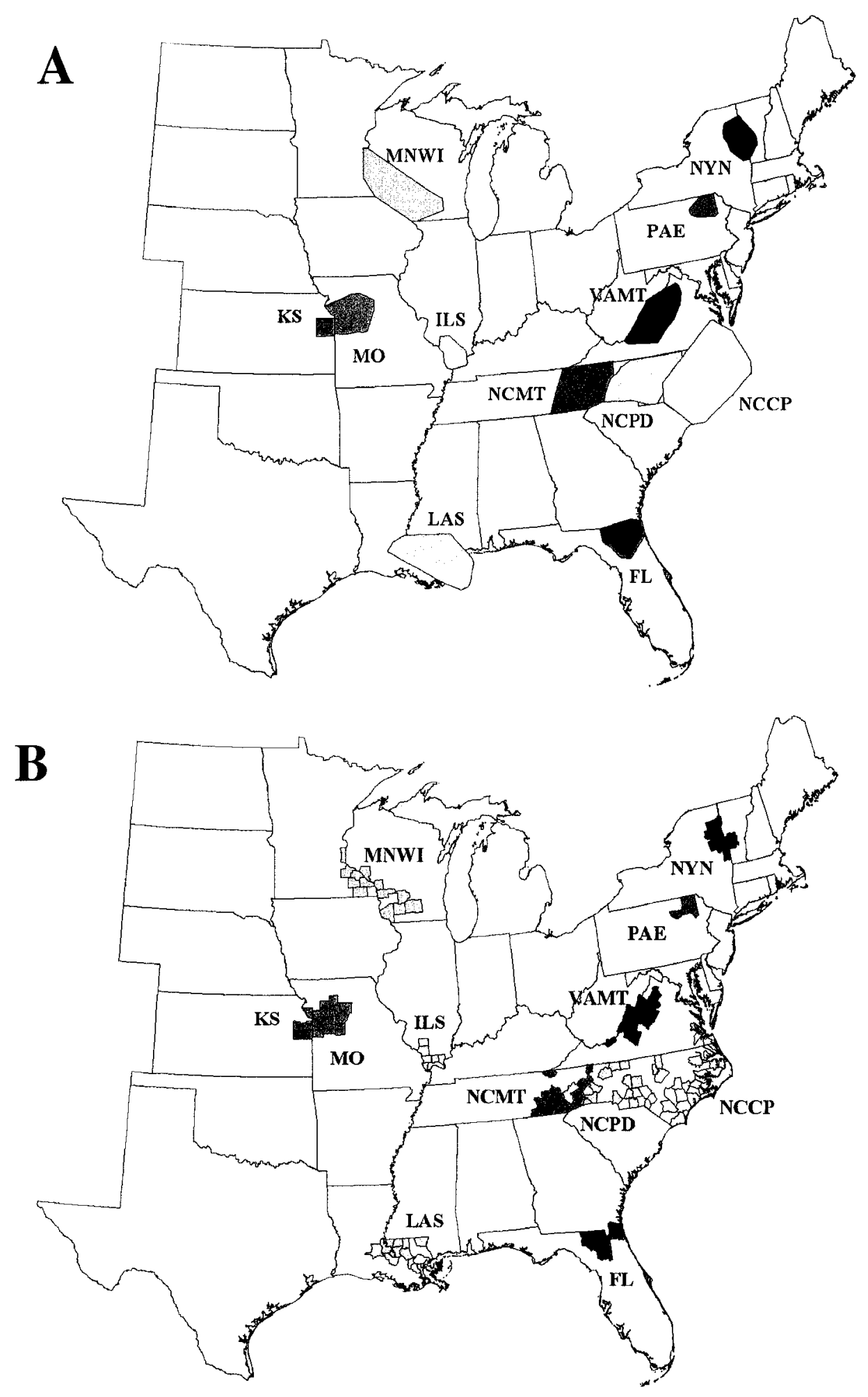

Fig. 5.-The 12 selected study areas used for several analyses. (A) Map of the 12 selected study areas. Shapes of study areas represent minimum convex polygons connecting outlying counties. (B) Map of study areas showing counties from which specimens were examined. 
Spatial analysis of coloration was performed for combined sexes using ArcView, version 3.2. Each coloration character was classified into four qualitative states (Appendix II). For each character, the state exhibited by each specimen was joined to the GIS by county using the Federal Information Processing Standards (FIPS) codes. The states of each coloration character were individually mapped on the same county base map of the United States to examine geographic patterns. Because many counties contained numerous specimens exhibiting different states of the same character, additional maps were prepared to show the percentages of each coloration state within the 32 geographic regions.

\section{Regression Analysis of Meristic Data}

Six regression analyses were performed to explore morphological relationships among meristic characters related to the number, spacing, and size of body crossbands and blotches. The regressions (Y on $\mathrm{X}$ ) were $\mathrm{BS}$ on $\mathrm{BL}, \mathrm{TE}$ on $\mathrm{BL}, \mathrm{TE}$ on $\mathrm{BS}, \mathrm{TE}$ on $\mathrm{VS}$, CS on VS, and BCB on TE (see Appendix II for descriptions of characters). Separate regressions were computed for males and females. The residuals were computed to test for heteroscedasticity and to expose patterns in the data. Each relationship was tested for sexual dimorphism by analysis of covariance (ANCOVA) using the SAS GLM procedure. The classification variable was sex and the independent variable was the covariate. The interaction term, covariate* sex, was tested in the first model. If the interaction was not significant, this term was removed and the effect of sex could be tested. If the interaction was significant, then the effect of sex could not be tested alone. 


\section{Principal Component Analysis of Meristic Data}

Multivariate analysis of the meristic data was used to determine whether distinct geographic units exist within Crotalus horridus. Three independent multivariate analyses were conducted separately on data for males, females, and combined sexes. Logarithmic transformation was used on the 24 meristic characters to remove excessive skewness, nonnormality, and heteroscedasticity. The following variables were removed from the analysis because high skewness, nonnormality, or extremely low variances remained even after logarithmic transformation or because of systematic errors in data collection: DCS, DMB, BCB, HB, UB, YB, TCB, and MSW. Principal component analysis (PCA) was performed using the SAS PRINCOMP procedure (with the covariance matrix option) on 16 meristic characters to identify the most important characters for differentiating individuals. PCA reduced the dimensionality of the data and concentrated the total variation into a smaller subset of variables for subsequent cluster analysis.

\section{Cluster Analysis of Meristic Data}

Cluster analysis was performed using the SAS CLUSTER procedure on the first 10 principal components of each model to create an original classification with no $a$ priori groups. That is, the county data were not grouped into predetermined regions, as they were in the analysis of individual meristic characters. On the contrary, the cluster analysis was conducted without regard to locality, so the models did not assume any underlying geographic pattern. The cluster analysis was used to determine where discrete 
clusters occur in morphological space (Sneath and Sokal, 1978), rather than geographic space. I used Ward's minimum-variance method to perform the cluster analysis (Ward, 1963). Ward's is a hierarchical clustering method that agglomerates individual observations into homogeneous groups by minimizing the within-group sum of squares (Anderberg, 1973; Everitt et al., 2001; Khattree and Naik, 2000; Sharma, 1996). The distance between clusters is measured by the between-group sum of squares. Independent cluster analyses were conducted separately on males, females, and combined sexes.

Seven diagnostic statistics were computed to evaluate the cluster solution and to determine the number of clusters for each model. Those statistics consisted of the cubic clustering criterion (CCC), pseudo $t$-squared statistic (PSTSQ), pseudo F-statistic (PSF), semipartial R-squared statistic (SPRSQ), R-squared statistic (RSQ), between-group sum of squares (BSS), and root-mean-square standard deviation (RMSSTD). Each statistic was plotted against the number of clusters and evaluated for sharp peaks or angles associated with large changes in value that identify possible cluster solutions (Everitt et al., 2001; Khattree and Naik, 2000; Sharma, 1996). Cluster solutions are indicated by high values of RS and BSS, and by low values of CCC, PSTSQ, PSF, SPRSQ, and RMSSTD. Because each statistic identifies several possible cluster solutions and the sampling distribution of each statistic is not known, the number of clusters for further study was determined by reaching a consensus among all seven statistics. The number of clusters for combined sexes was five, so cluster analyses were performed on all models from the five-cluster to the two-cluster model, to examine how clusters merged into each other. The same analyses were also performed separately on males and females. 
A dendrogram was produced for each model using the SAS TREE procedure. The dendrograms show graphic displays of the agglomerative clustering process. Individual observations on the horizontal axis are grouped hierarchically into larger clusters using the between-group sum of squares (BSS), which is plotted on the vertical axis as a distance measurement. The dendrogram can be cut horizontally at regions that exhibit a sharp increase in BSS, which identifies possible cluster solutions. The SAS TREE procedure does not label each cluster, nor does it indicate which variables are most responsible for each node in the dendrogram. However, canonical discriminant analysis was performed for each cluster solution to characterize the multivariate morphospace of each cluster. The canonical variables were computed using the SAS CANDISC procedure, and all combinations of the canonical variables were plotted in two or three dimensions for each model.

The question arises whether the results change drastically with small changes in the cluster analysis program. Therefore, additional cluster analyses were conducted to investigate the robustness of the clustering procedure to alternative programs or different analytical options. For example, I varied the number of principal components and performed the cluster analysis on the raw data using Ward's method. The results of those tests were consistent with the primary analysis. I also eliminated redundancy in several character suites by combining correlated variables into a mean value for that character (e.g., right and left labial scales) and performing the analysis again with the new composite variables. These analyses also did not yield substantially different results. Other methods of cluster analysis were tested (SAS, 1989), and their diagnostic statistics and canonical variable plots were compared to those produced by Ward's method. The 
complete linkage method (Sorenson, 1948) and McQuitty's similarity analysis (McQuitty, 1966; Sokal and Michener, 1958) performed similarly to Ward's method and produced five and three large overlapping clusters, respectively, using the five-cluster solution and two clusters each using the two-cluster solution. The median method (Gower, 1967) produced two large clusters and one extremely small cluster with even higher levels of overlap using the five-cluster solution, and only one cluster using the two-cluster solution. The average linkage method (Sokal and Michener, 1958) produced two large clusters with more separation than the other methods using the five-cluster solution and only one cluster using the two-cluster solution. The centroid method (Sokal and Michener, 1958) did not distinguish separate clusters using any cluster solution.

\section{Discriminant Function Analysis of Meristic Data}

Discriminant function analysis (DFA) was performed using the SAS DISCRIM procedure to test each classification produced by cluster analysis, by reclassifying each observation into a cluster group. The reclassifications were compared to the original cluster designations to determine how well the original model was supported by the DFA. The homogeneity of the within-group covariance matrices was tested using the likelihood ratio chi-square statistic. Prior probabilities were set in proportion to the sample sizes of each cluster. The generalized squared distances between groups were computed to show the distance between each pair of clusters. The crossvalidation option was used to compute the crossvalidation error rates using a quadratic discriminant function because the covariances were not homogeneous. The crossvalidation option produces a nearly 
unbiased estimate of the number of misclassifications of each group (SAS, 1989). The number of misclassifications is used to compute the group-specific error-count estimates and the total error-count estimate for each model. Separate DFAs were performed to test the previous classifications of Gloyd (1940) and Martin (1992a) by grouping specimens $a$ priori by nominal subspecies or regional morphs, respectively. The results of those tests were compared to the cluster models by examining the crossvalidation error rates.

\section{Geographic Analysis of Multivariate Clusters}

Spatial analysis of each cluster model was performed using a geographic information system (GIS; ArcView, version 3.2, Environmental Systems Research Institute (ESRI), 1992-1999). For each model, the cluster designation for each specimen was recorded from SAS and associated with the corresponding county designation. The cluster designation for each specimen was joined to the GIS using the county FIPS codes. Each cluster was mapped individually on a county base map of the United States using the ArcUSA 1:2M data set (ESRI, 1997) to determine whether any clusters exhibited geographic cohesiveness. Because many counties contained specimens belonging to different clusters, additional maps were prepared to show the percentages of each cluster within each of the 32 previously defined regions. The regional classification was used $a$ posteriori for this analysis and did not affect the actual clustering procedure. The maps were interpreted subjectively by visual inspection. 


\section{Analysis of Morphometric Data}

Means of selected morphometric characters were computed separately for males and females greater than $800 \mathrm{~mm}$ SVL using the SAS MEANS procedure. The calculations were made in 12 selected study areas (Fig. 5). Analysis of variance was performed using the SAS GLM procedure to determine whether significant differences existed among the 12 study areas. The effect of region was tested using Tukey's studentized range test to determine which regions were significantly different from others. Regional groups identified by Tukey's tests were listed in order from the largest to the smallest means.

Analysis of covariance (ANCOVA) was used to investigate the presence of sexual dimorphism and geographic patterns in the morphometric data. This analysis was completed in three parts. It was assumed that the large sample size would inflate the differences between males and females by making some small differences significant, so the effect of sex was tested in a hierarchical manner to determine the strength of sexual dimorphism in each relationship.

Part 1.--Regression analyses were performed using the SAS REG procedure on the entire data set to examine 10 relationships of body, tail, and head measurements: TL on SVL, HLM on SVL, HLS on SVL, QL on SVL, ML on SVL, HLS on HLM, HW on HLM, QL on HLM, ML on HLM, and QL on ML. Separate regressions were fitted for males and females for each relationship. The residuals were computed to test for heteroscedasticity and to expose patterns in the data. ANCOVA was performed using the SAS GLM procedure to test for the effect of sex. Depending upon the regression, the 
covariate was either SVL, HLM, or ML. The covariate* sex interaction term was tested first. If the interaction was not significant, then the interaction term was removed from the model and the effect of sex was tested directly.

Part 2.- Separate regression analyses were performed on the combined data set for the 12 selected study areas (Fig. 5), examining the same 10 relationships as in Part 1. Separate regressions were fitted on males and females for each relationship. ANCOVA was performed on the combined data set for the 12 study areas. If either the interaction term or the effect of sex was significant, then separate analyses were performed on males and females in Part 3. If the effect of sex alone was not significant, then one analysis was performed on combined sexes in Part 3.

Part 3.-ANCOVA was performed either on males and females separately, or on the combined sexes to test for the effect of geographic region in each regression, using the data for the 12 selected study areas. The sex classes depended on the results of significance tests for the effect of sex in Part 2. The same covariates were used for each regression as in Part 2, but region was used as the classification variable. The covariate*region interaction was tested first. If the interaction term was not significant, then the effect of region could be tested using the REGWQ multiple range test, to determine which regions were significantly different from others. Regional groups identified by the REGWQ tests were listed in order from the largest to the smallest means of the dependent variable. If the covariate*region interaction was significant, then the effect of region could not be tested directly.

Factor analysis was performed using the SAS FACTOR procedure on the morphometric data as an additional method to determine whether the morphometric data 
exhibit distinct geographic units. Factor analysis can be used to identify common factors among a set of measurements that reflect an underlying structure (SAS, 1989; Khattree and Naik, 2000). The first common factor usually represents overall size. Subsequent factors usually describe different shape attributes revealed by the analysis. Principal factor analysis was performed with the minimum eigenvalue set at 1.0, to limit the model to factors that account for the most variation. Analysis of variance was used to determine whether the important size and shape factors resulting from the factor analysis vary significantly over the geographic range of the species. In general, the regional classification variable was coded using the 32 regions defined in the univariate analyses, although some of the smaller regions were pooled with larger regions to accommodate the assumption of homoscedasticity required for the ANOVA. Multiple range tests (REGWQ) were performed on the factor scores to determine which regions were significantly different from each other. 


\section{RESULTS}

\section{Univariate Analysis of Meristic Data}

Means, descriptive statistics, and ANOVA results for 24 meristic characters were computed in all 32 regions for males (Appendix IV), females (Appendix V), and combined sexes (Appendix VI). Eight variables had high skewness, extremely low variances, or nonnormality, even after logarithmic transformation: DCS, DMB, BCB, HB, UB, YB, TCB, and MSW (see Appendix II for descriptions of characters). The remaining characters displayed significant levels of geographic variation $(P<0.0001)$. Two sample $t$-tests using all data detected significant sexual dimorphism $(P<0.01)$ in the following characters: ADS, MDS, VS, CS, LSL, RSL, LDB, RDB, LVB, RVB, TE, BL, and BS. Males had larger values for ADS, MDS, CS, LDB, RDB, LVB, RVB, and TE, whereas

females had larger values for LSL, RSL, VS, BL, and BS. However, the magnitude of the differences between males and females was large only for CS and BL, moderate for VS and BS, and very small for the rest of the sexually dimorphic characters.

Similarly, $t$-tests on data from the 12 selected study areas (Fig. 5) detected significant sexual dimorphism $(P<0.05)$ in the following characters: VS and CS (Northern New York and Vermont); VS and CS (Eastern Pennsylvania); MDS, VS, and CS (Virginia Mountains); VS, CS, LSL, RDB, and BL (North Carolina Piedmont); VS, CS, LSL, RSL, and BS (North Carolina and Virginia Coastal Plain); VS, CS, BL, and BS (North Carolina Mountains); VS, CS, LSL, and RSL (Florida); VS and CS (Minnesota and Wisconsin); ADS, VS, CS, LSL, LIL, BL, and BS (Eastern Kansas); ADS, VS, CS, 
LSL, RSL, and BL (Northwestern Missouri); VS, CS, and BL (Southern Illinois); VS, $\mathrm{CS}$, and BL (Southern Louisiana). In general, these results indicate that males have more subcaudal scales and females have more ventral scales. Males have slightly more midbody dorsal scales and females have longer band length and band spacing.

\section{Geographic Analysis of Meristic Data}

Meristic characters showed several patterns of geographic variation. Dorsal scale rows exhibited lower mean values in the northern regions and higher mean values in the southern regions (Fig. 6). The division between the northern and southern regions was located through southern Kansas and southern Missouri, northward into southern Illinois, then southward across Kentucky and Tennessee, around the southern Appalachian Mountains, northeast along the Appalachians in North Carolina, and east along the southern border of Virginia. Southern Iowa had unusually high values of all dorsal scale rows compared to the surrounding regions. The low means for midbody dorsal scales (MDS) in the north extended deep into the southern Appalachians, whereas the low means for anterior dorsal scales (ADS) and posterior dorsal scales (PDS) in the north reached only into southwestern North Carolina. Although PDS exhibited very low variance, it showed the same north-south pattern of geographic variation as ADS and MDS. PDS only deviated from MDS by having lower values in the North Carolina Piedmont and higher values in the southern Appalachians. Separate analyses of males and females for MDS gave very similar results to those for combined sexes, except that males had relatively higher values in the southern Appalachians. 

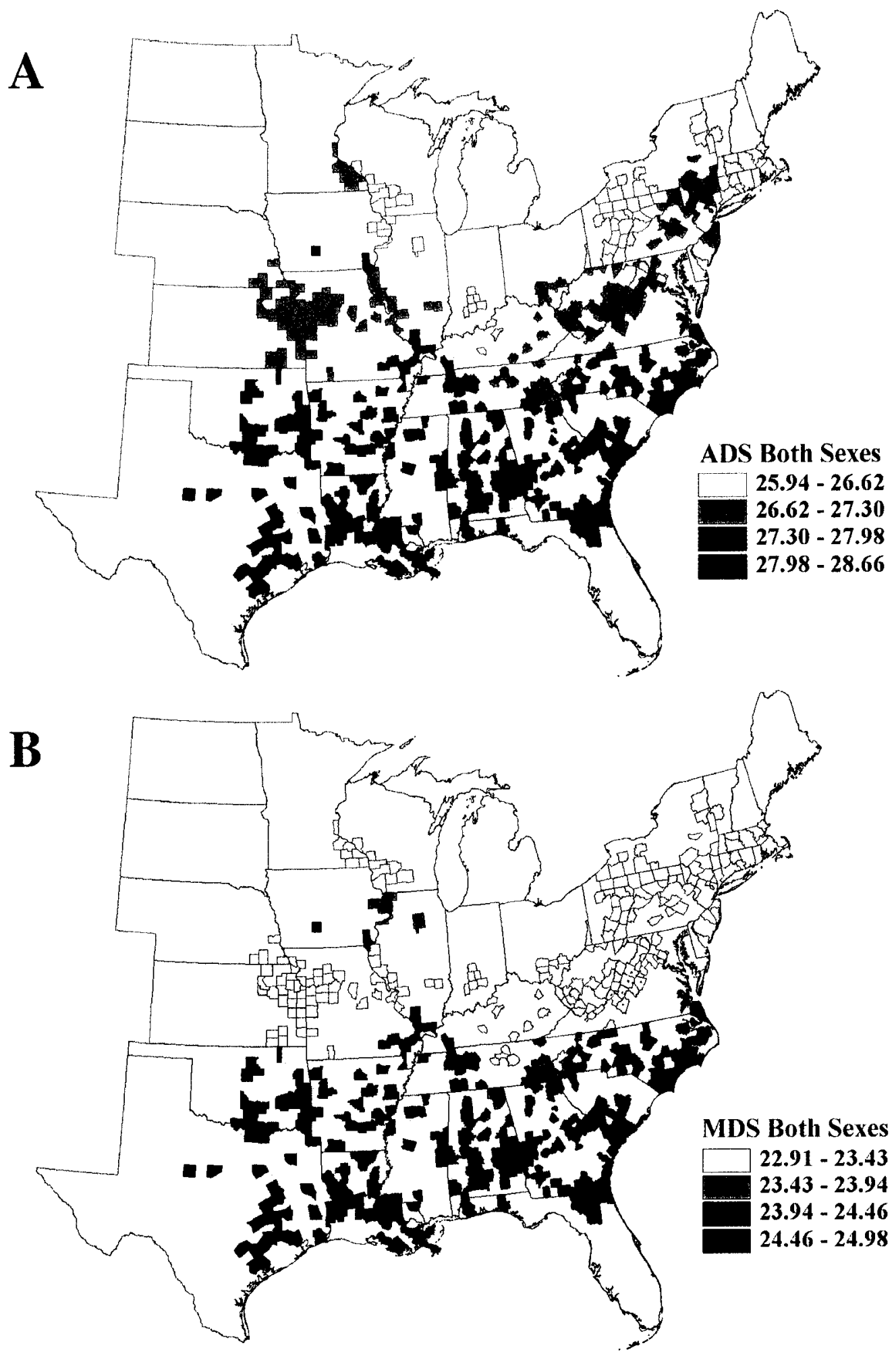

Fig. 6.-Geographic variation in number of dorsal scale rows in 32 regions. (A) Anterior dorsal scales. (B) Midbody dorsal scales. Color of county reflects mean value of region to which it belongs. Descriptions of characters are provided in Appendix II. 
Male and female ventral scales (VS) showed a similar pattern to combined-sex MDS, with low values in the northern regions and high values in the southern regions (Fig. 7A,B). However, VS of males deviated from the pattern for MDS by exhibiting high values of VS in Kansas, Nebraska, western Illinois, and moderately high values in northern New York and New England. Females deviated from the MDS pattern by having high values of VS in Wisconsin and northern New York and low values in Alabama and Mississippi. Male and female subcaudal scales (CS) differed greatly from each other (Fig. 7C,D). Male CS generally showed the same north-south pattern as MDS, but deviated from that pattern by exhibiting low values of CS in Georgia, western Kentucky, western Tennessee, and the North Carolina Piedmont and high values in northern New York and western Illinois. Female CS showed a very different pattern, with low values in the northern, central, and southeastern regions and high values concentrated in the southwestern regions. Southern Iowa did not show unusually high values of VS or CS.

Labial scale counts showed two different patterns of geographic variation (Fig. 8). Supralabials exhibited low values in the northwestern regions and high values throughout the rest of the range, although lower intermediate values occurred among a few northern regions, notably Missouri and the central Appalachians. Infralabials showed low values in the northwestern and northeastern regions and high values in the north-central and southern regions. However, there is evidence of a north-south cline in infralabials, especially in the western regions.

Transverse elements (TE) and five characters related to body blotch counts showed a consistent pattern of high values in the southern, northwestern, and central- 

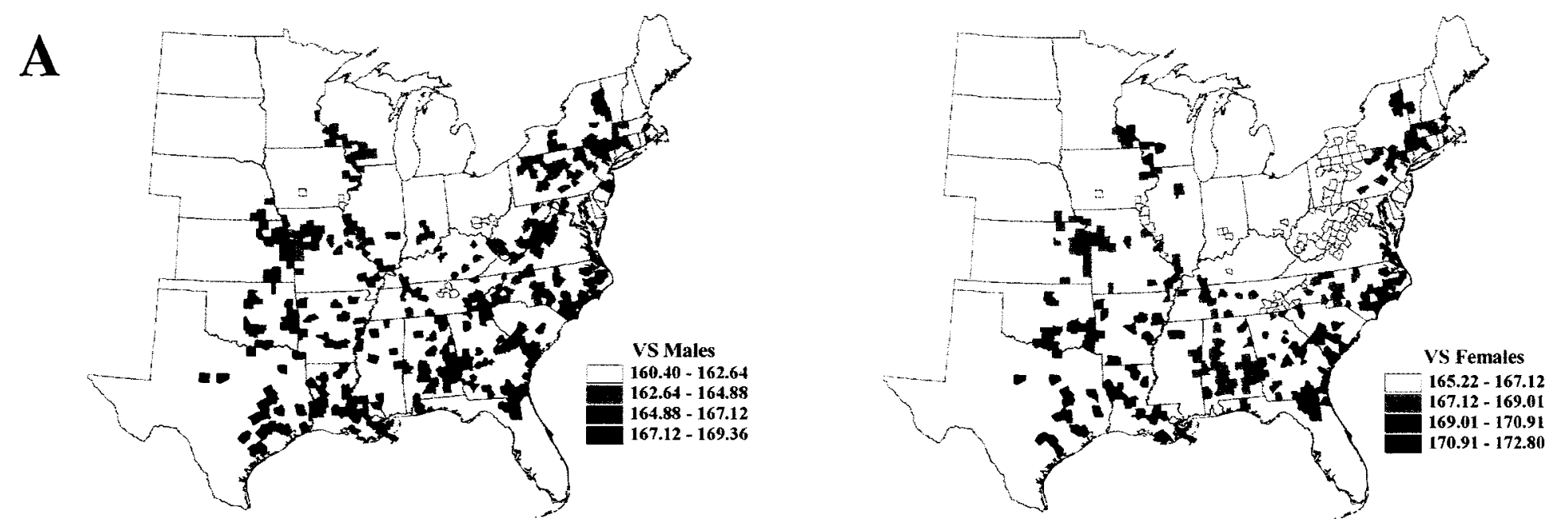

B
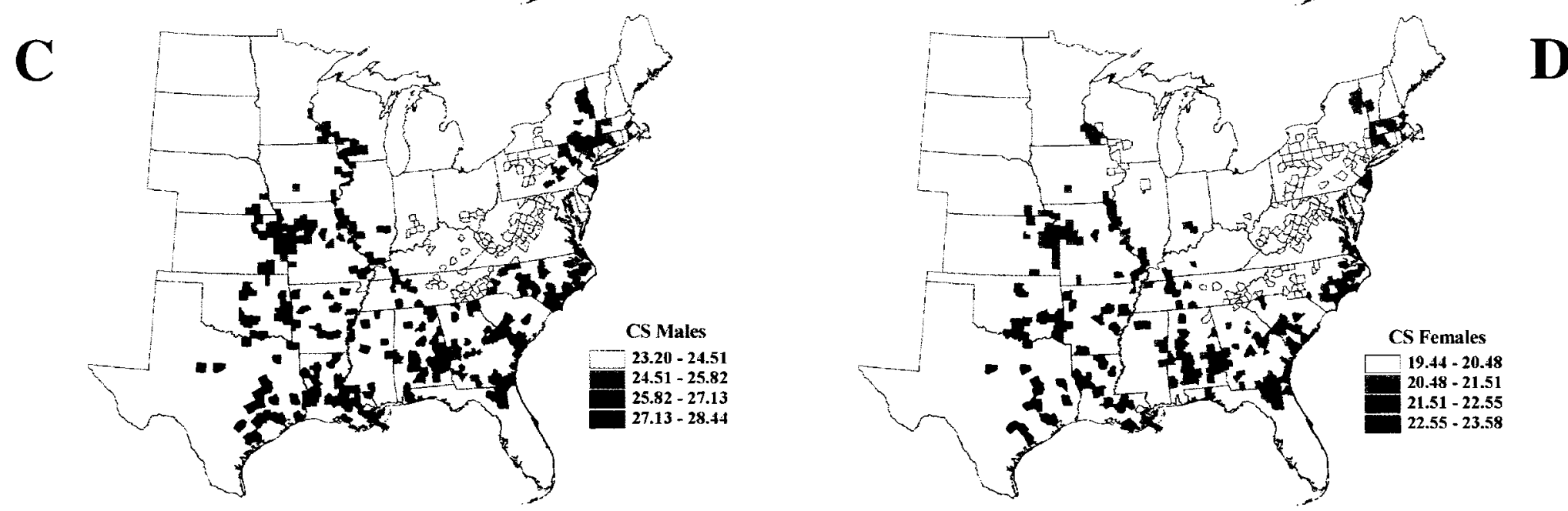

Fig. 7.-Geographic variation in ventral and subcaudal scales in 32 regions. (A) Ventral scales of males. (B) Ventral scales of females. (C) Subcaudal scales of males. (D) Subcaudal scales of females. Color of county reflects mean value of region to which it belongs. Descriptions of characters are provided in Appendix II. 

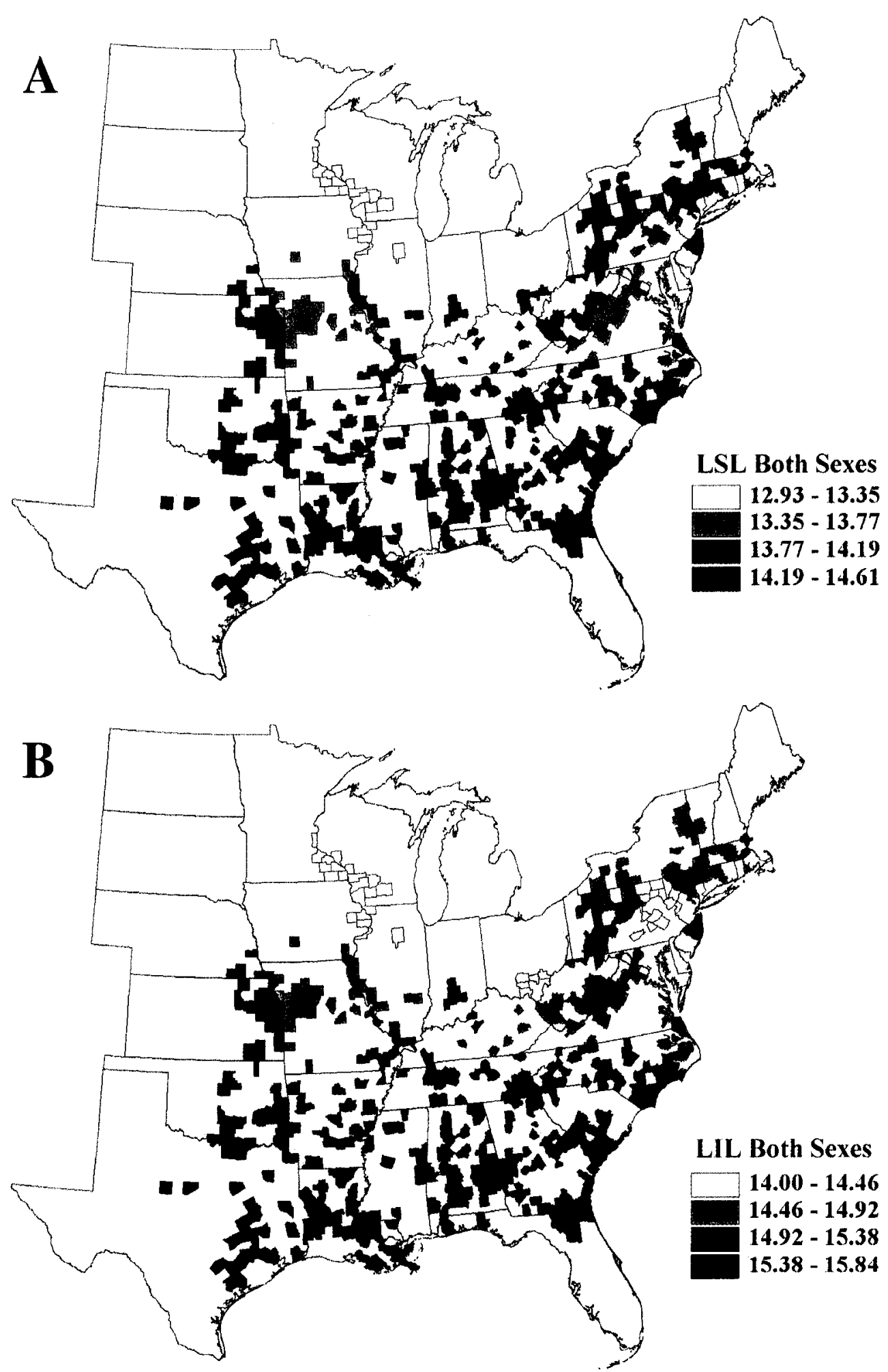

Fig. 8.-Geographic variation in number of labial scales in 32 regions. (A) Left supralabial scales. (B) Left infralabial scales. Color of county reflects mean value of region to which it belongs. Descriptions of characters are provided in Appendix II. 
western regions, as well as southern Ohio and northern New York; low values occurred in the northeastern, central-eastern, and north-central regions. Deviations from the TE pattern for body blotches occurred in southern Ohio; left lateral blotches had lower values than right lateral blotches. TE deviated from the DMB pattern by exhibiting low values in southern Indiana, central Kentucky, and central Tennessee and high values in southern Ohio (Fig. 9A,B). Within the northeastern regions, northern New York had conspicuously high values in TE and all body blotch characters. Separate analyses of TE in males and females revealed minor deviations from the patterns of combined sexes; male TE showed low values in western Illinois, southern Indiana, and central Kentucky, whereas female TE showed high values in those regions.

Body crossbands (BCB) showed another pattern of geographic variation with high values concentrated largely in the southeastern regions. Male BCB had high values in the southeastern regions from the mid-Atlantic Coastal Plain to northern Mississippi, and in central Tennessee, Minnesota, and Wisconsin (Fig. 9C). Female BCB had high values in the southeastern regions from the mid-Atlantic Coastal Plain to Georgia and northeastern Alabama, and in Minnesota, Wisconsin, northern Illinois, western Iowa, eastern Pennsylvania, northwestern New Jersey, and the New Jersey Coastal Plain (Fig. 9D).

Band length (BL) and band spacing (BS) showed a pattern in which the northeastern and central-eastern populations resembled some populations in the northcentral and central-western regions depending upon the sex. Males exhibited high values of $\mathrm{BL}$ in the northeastern and central-eastern regions, south along the Appalachians into eastern Tennessee and western North Carolina, and west of the Appalachians into southern Ohio, with lower values in the remaining regions (Fig. 10A). Females had high 
A

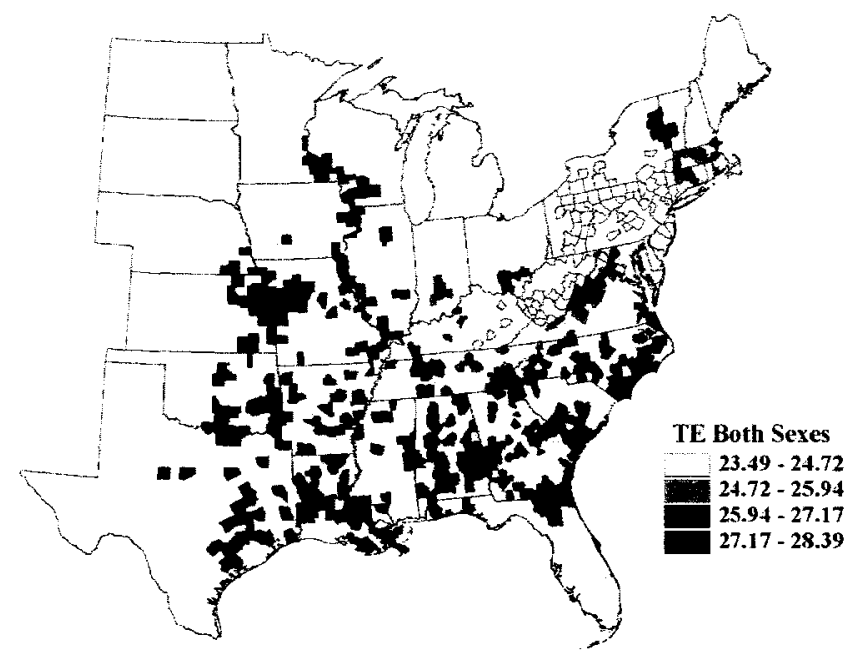

$\mathbf{C}$

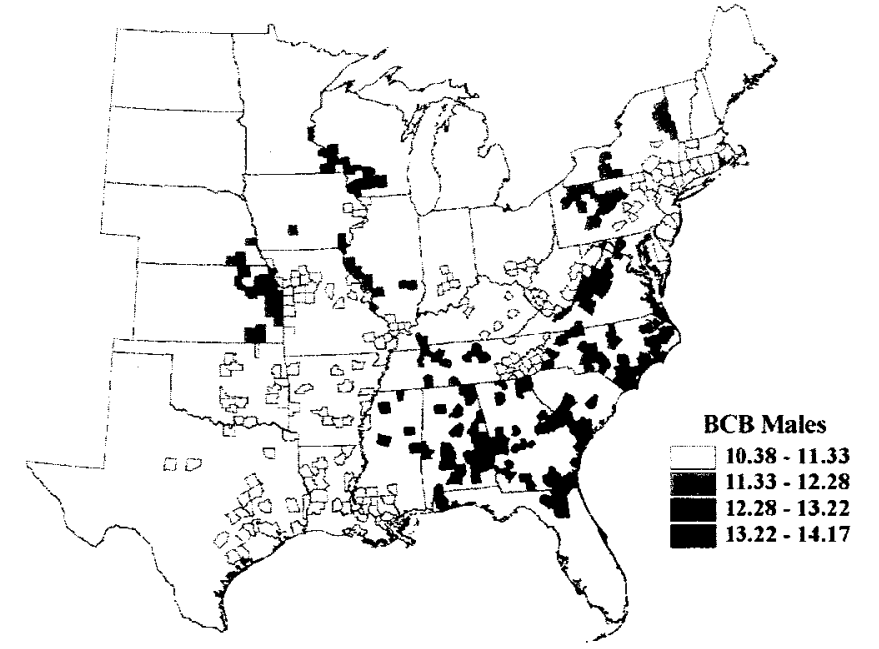

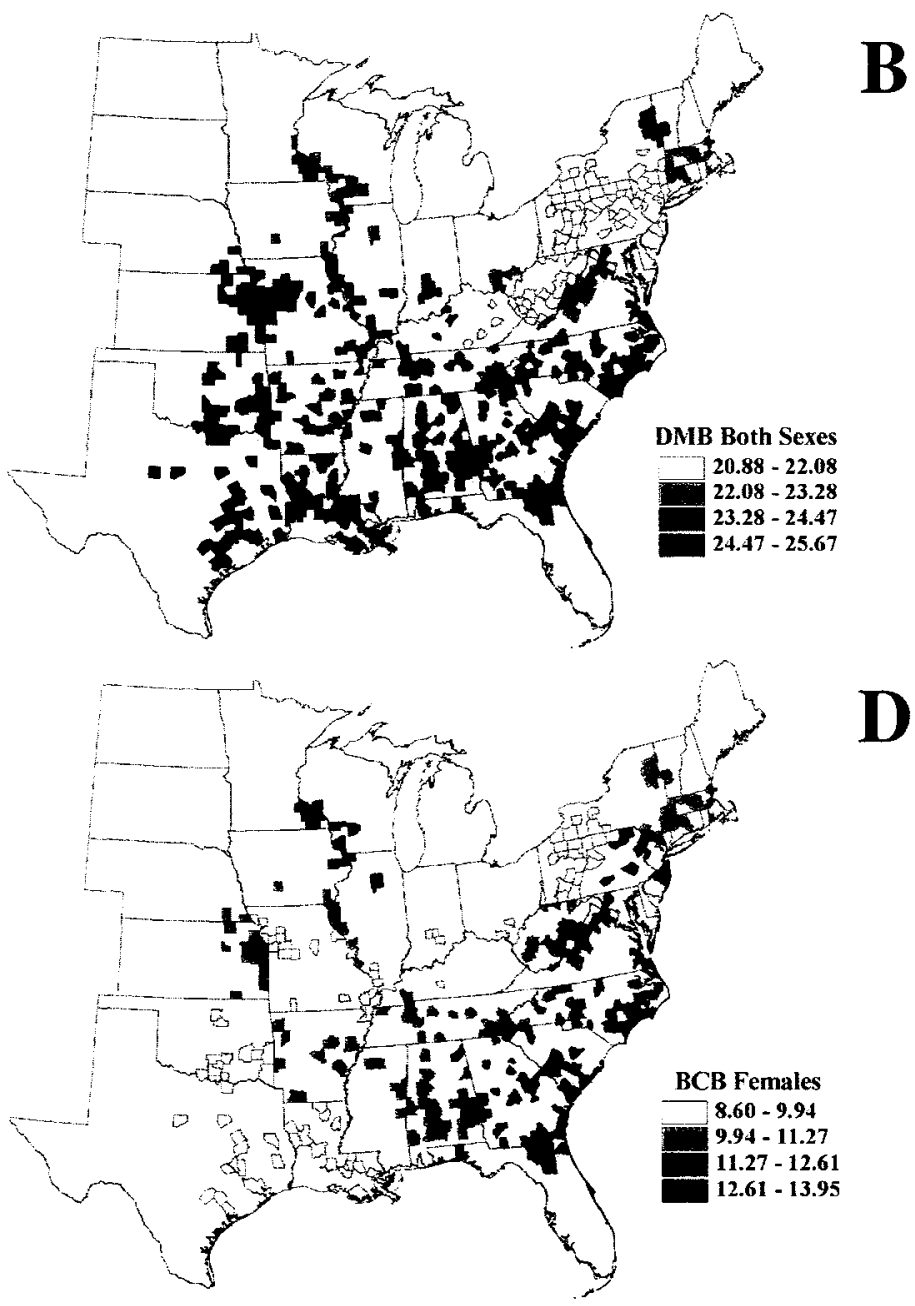

D

Fig. 9.-Geographic variation in dorsal pattern elements in 32 regions. (A) Transverse elements of combined sexes. (B) Dorsal midbody blotches of combined sexes. (C) Body crossbands of males. (D) Body crossbands of females. Color of county reflects mean value of region to which it belongs. Descriptions of characters are provided in Appendix II. 
values of $\mathrm{BL}$ in the northeastern and central-eastern regions and across the north-central regions into southern Illinois and southeastern Missouri (Fig. 10B). Males had high values of BS in the northeastern and central-eastern regions, south along the Appalachians into eastern Tennessee and western North Carolina, across most of the north-central regions, and into western and southern Illinios, southeastern Missouri, and southern lowa (Fig. 10C). Females exhibited high values of BS in the northeastern and central-eastern regions, south along the Appalachians into eastern Tennessee and western North Carolina, across the north-central regions, and into southern Illinois, southeastern Missouri, and Arkansas (Fig. 10D).

Half bands (HB), Y-bands (YB), and U-bands (UB) represent pattern anomalies and all occurred in very low frequencies, which resulted in highly skewed statistical distributions. Nonetheless, these characters showed interesting patterns of geographic variation. HB exhibited low values throughout most of the range and high values in Minnesota, Wisconsin, northern Illinois, western and southern Iowa, and Florida (Fig. 11A). Higher incidences of YB occurred in the northeastern and central-eastern regions from northern New York to southern North Carolina (Fig. 11B). The highest values for YB occurred in the New Jersey Coastal Plain. Although UB also exhibited low values throughout most of the range, high values were scattered throughout the northwestern and eastern regions.

Tail crossbands (map not shown here) showed low values in the northern regions and high values in the southern and central-western regions. Middorsal stripe width (map not shown here) exhibited low values in most of the northeastern regions (where the stripe itself is largely absent) and in Minnesota, with high values throughout the rest of 

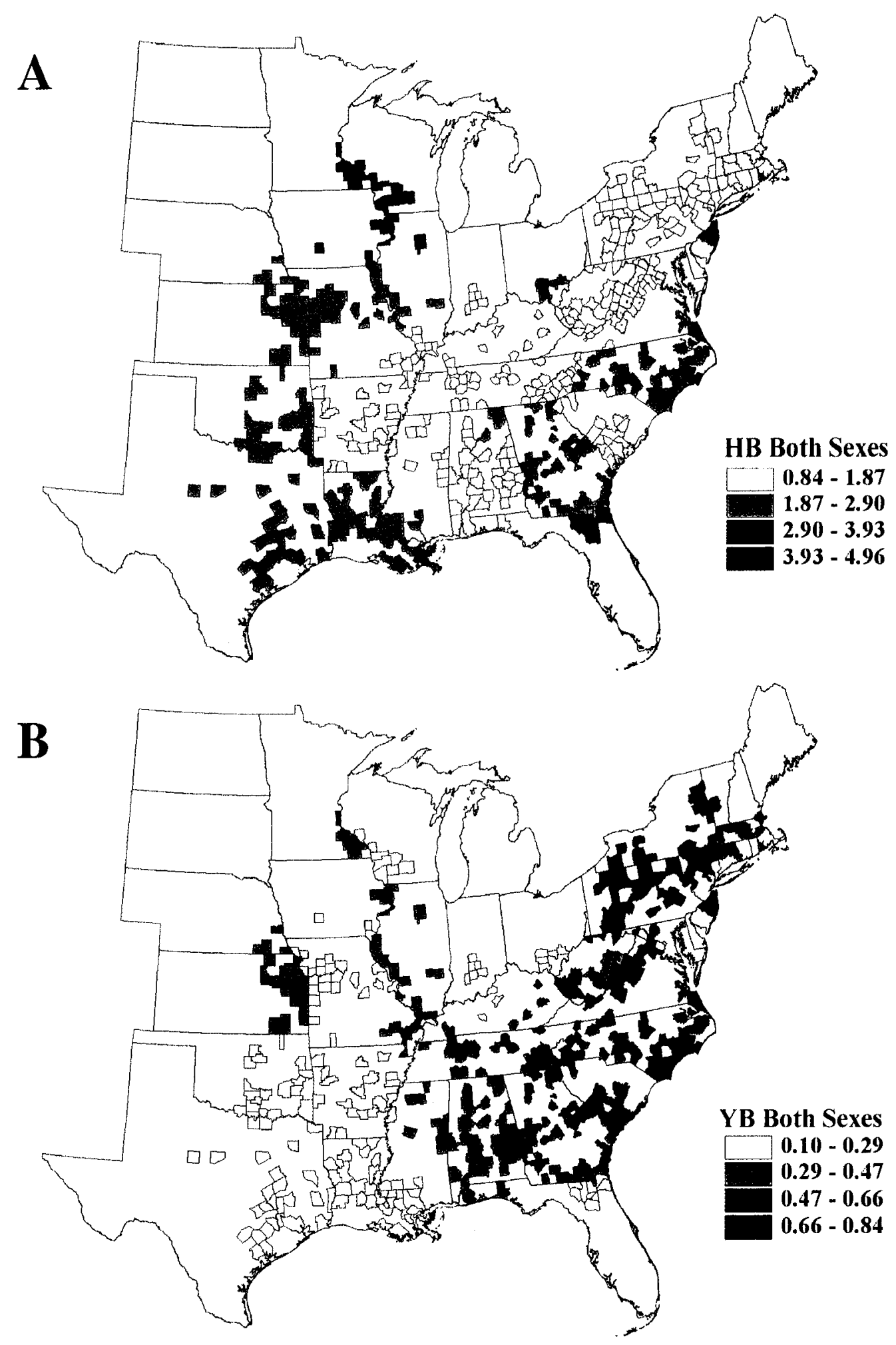

Fig. 11.-Geographic variation in number of irregular pattern elements in 32 regions. (A) Half bands of combined sexes. (B) Y-bands of combined sexes. Color of county reflects mean value of region to which it belongs. Descriptions of characters are provided in Appendix II. 
the sample range, including three northeastern regions (northern and eastern New York and West Virginia).

\section{Geographic Analysis of Coloration}

Melanin density (MD), ground color (GC), head color (HC), and postocular stripe (POS) consistently displayed distinctive states in the northeastern regions (Figs. 12-16, see Appendix II for descriptions of character states). Melanistic specimens only occurred from the northeastern regions to the Virginia Appalachians (Fig. 12). Specimens with high MD were concentrated from the northeastern regions to western North Carolina and eastern Tennessee and were distributed more moderately throughout the southern and central-western regions. Specimens with medium MD were concentrated in the southern, central-western, and central-eastern regions, moderately distributed in the northeastern regions, and scattered through the northwestern regions. Specimens with low MD occurred throughout the entire sample range. A map of MD as a percentage of regional populations revealed clinal variation among three broad areas (Fig. 16A). The northeastern and central-eastern regions were characterized by high $\mathrm{MD}$, the northwestern regions by low $\mathrm{MD}$, and the southern regions by low and medium $\mathrm{MD}$.

Black ground color occurred only from the northeastern regions to western North Carolina (Fig. 13). Specimens with dark GC were concentrated from the northeastern regions to western North Carolina and eastern Tennessee, the central-western and northwestern regions, and southwestern Oklahoma, with scattered individuals also in the north-central and southern regions. Individuals with moderate GC were concentrated in 
A

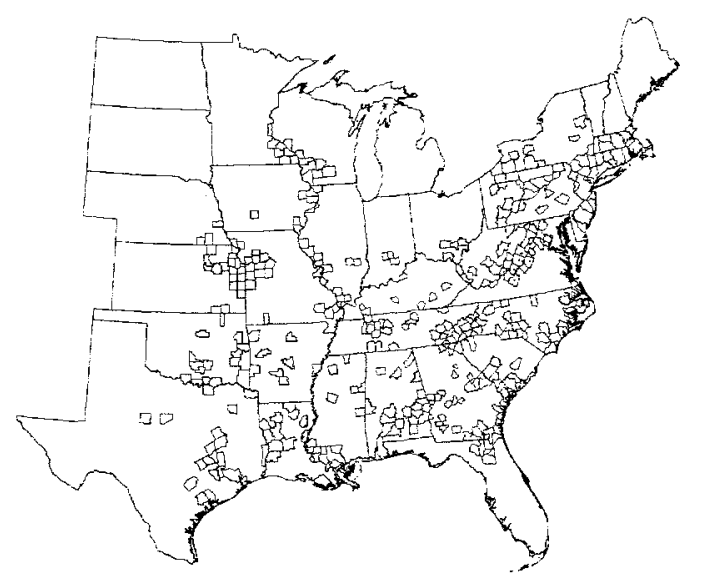

C

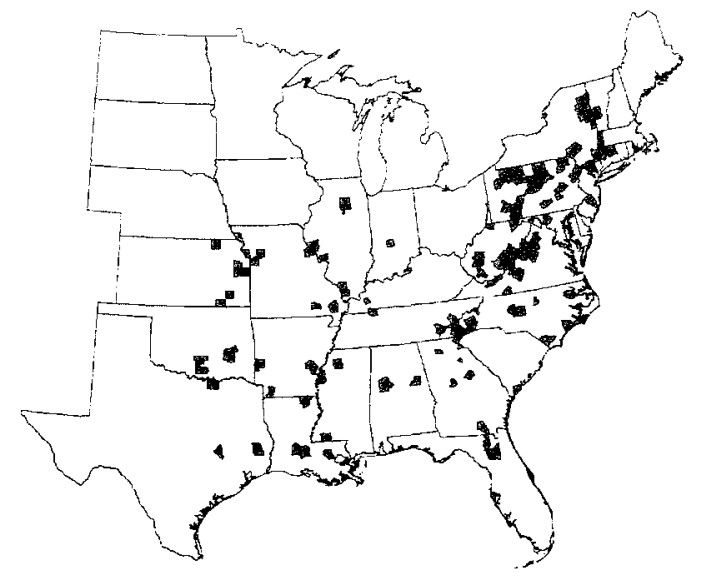

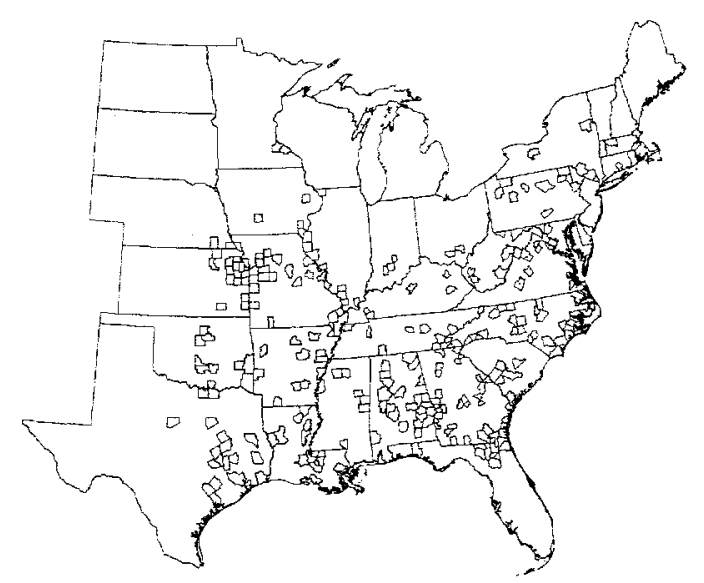

B

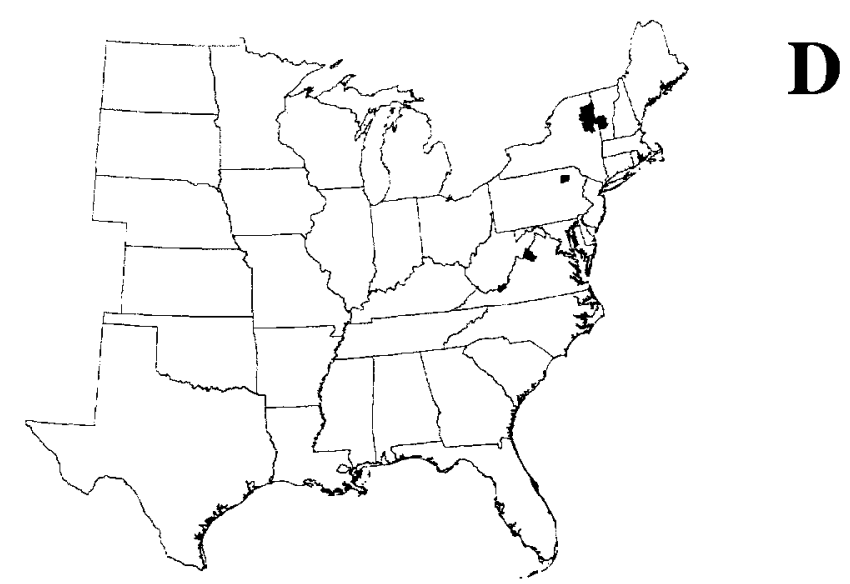

Fig. 12.-Geographic variation in melanin density. (A) Low. (B) Medium. (C) High. (D) Melanistic. Each character state of melanin density is mapped by county. Descriptions of character states are provided in Appendix II. 

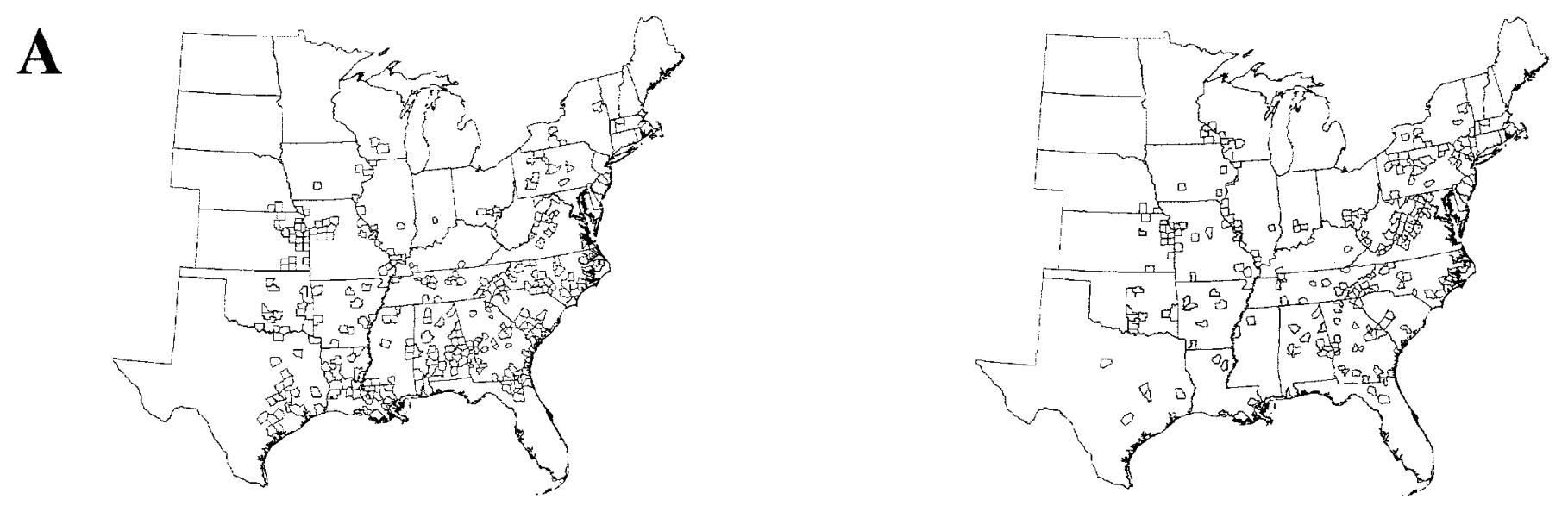

B
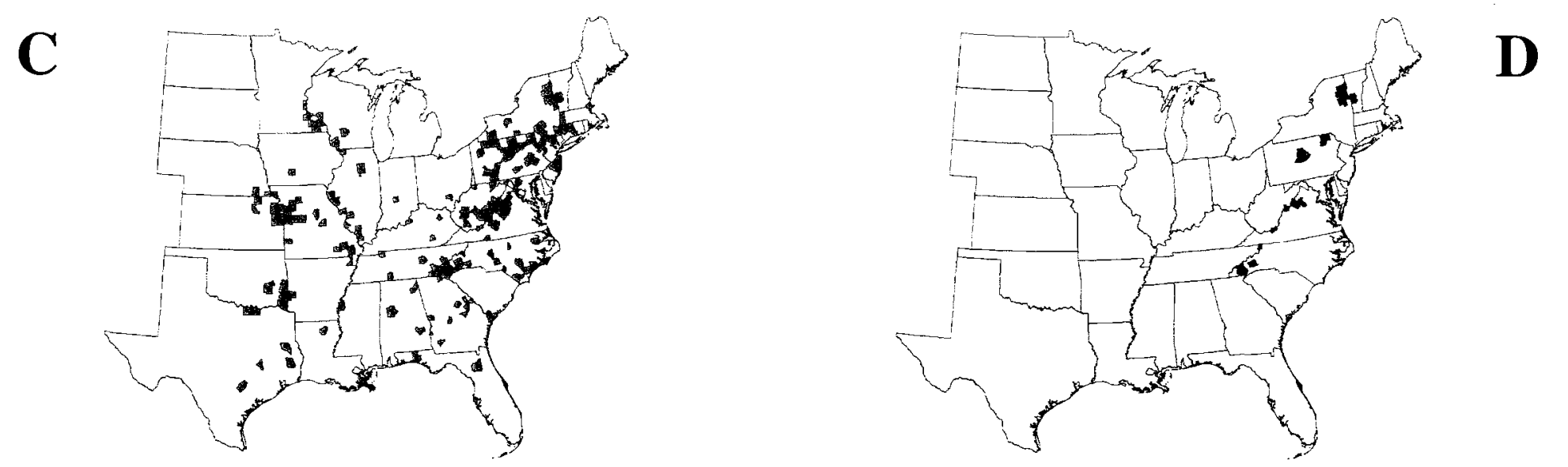

Fig. 13.-Geographic variation in dorsal ground color. (A) Light. (B) Moderate. (C) Dark. (D) Black. Each character state of ground color is mapped by county. Descriptions of character states are provided in Appendix II. 
A

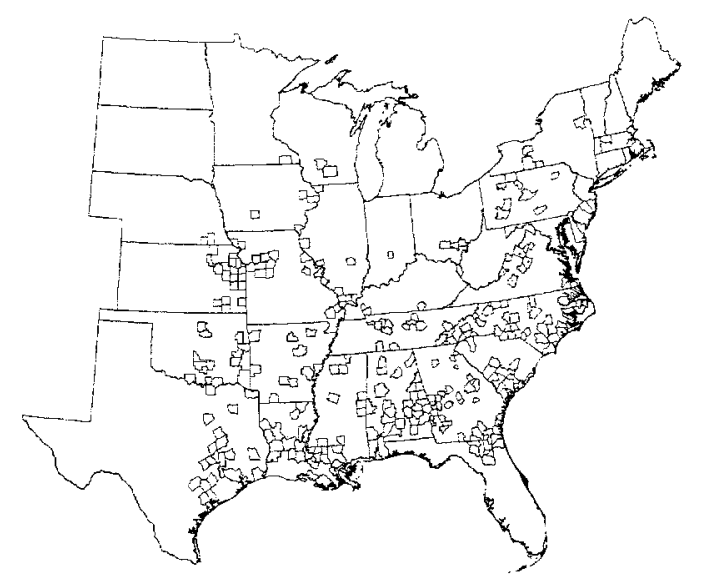

C

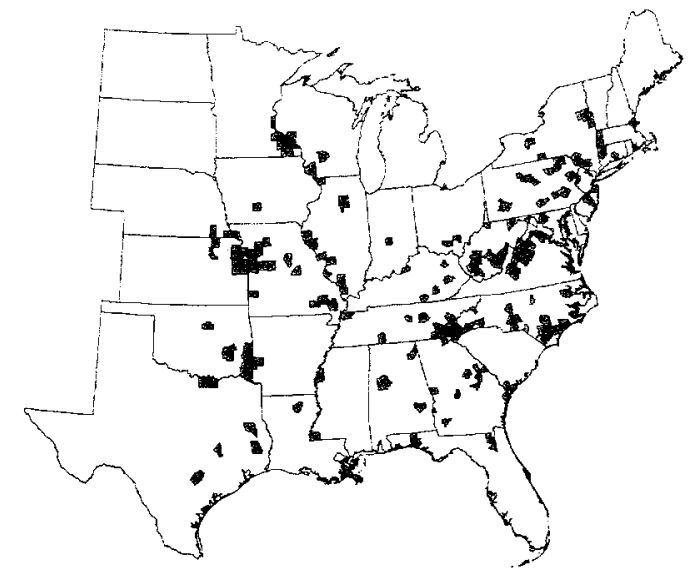

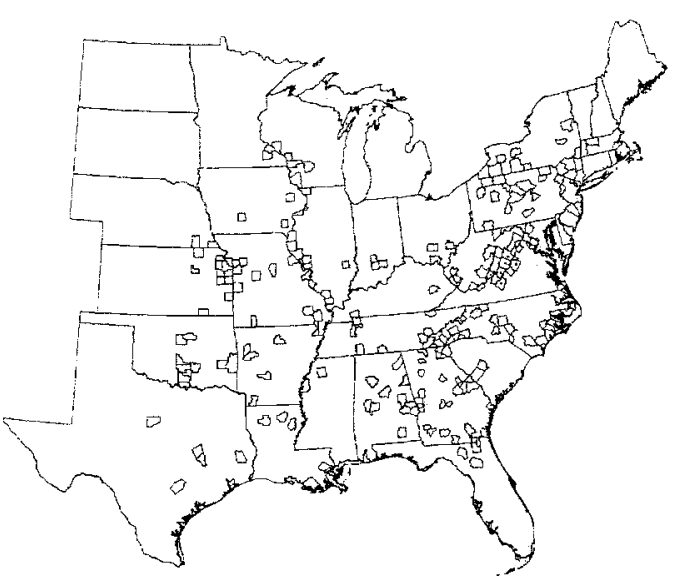

B

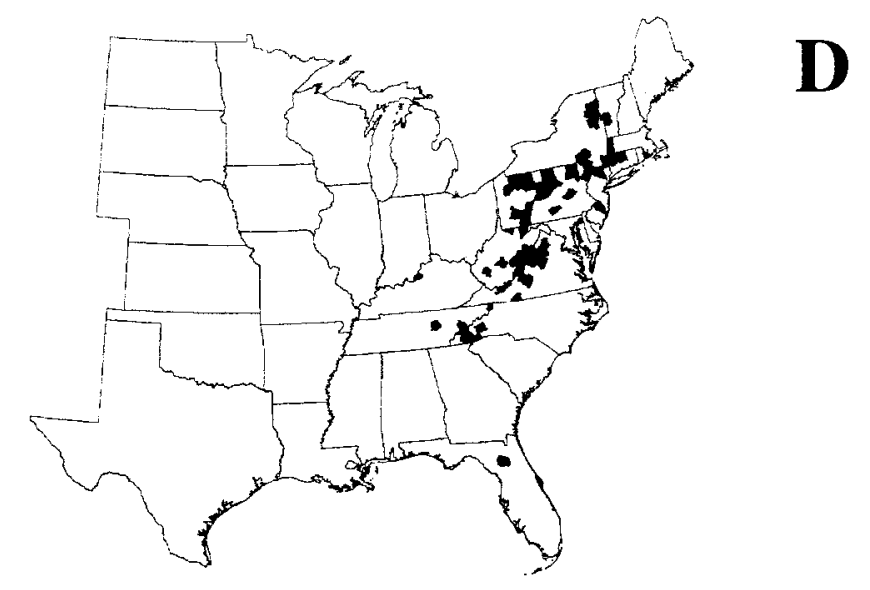

Fig. 14.-Geographic variation in dorsal head color. (A) Light. (B) Moderate. (C) Dark. (D) Black. Each character state of head color is mapped by county. Descriptions of character states are provided in Appendix II. 
A

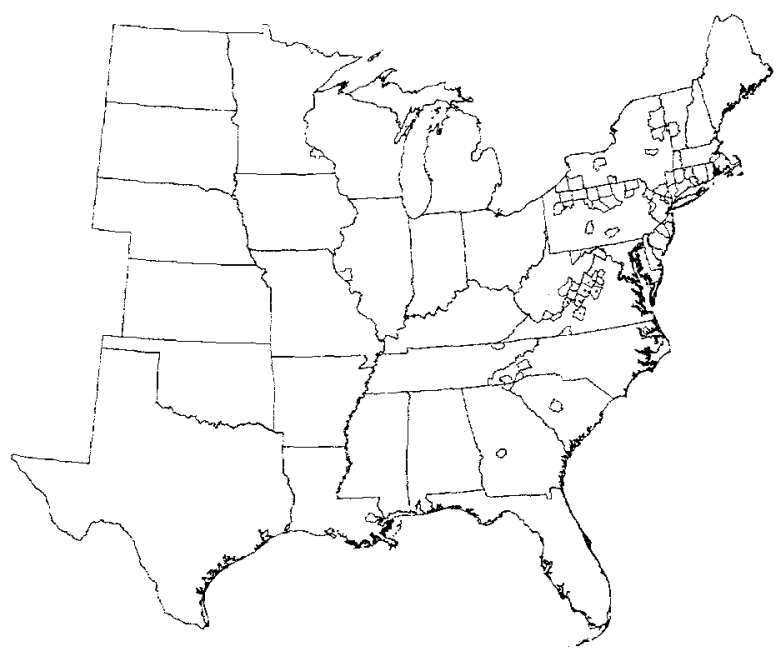

C

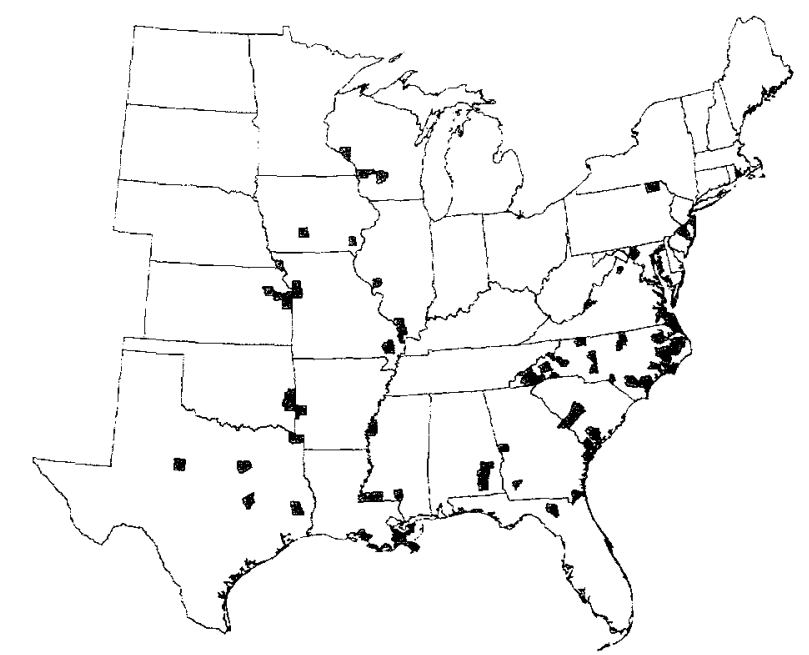

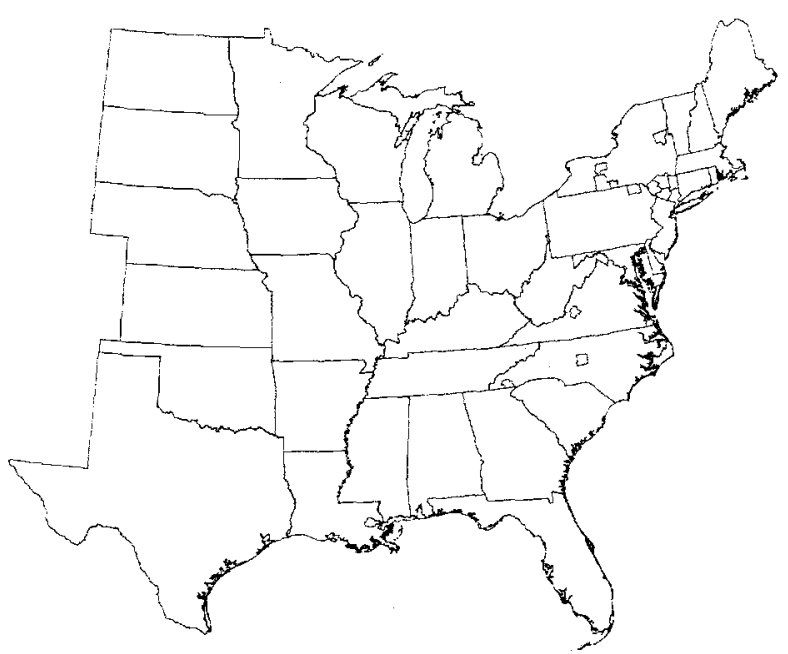

B

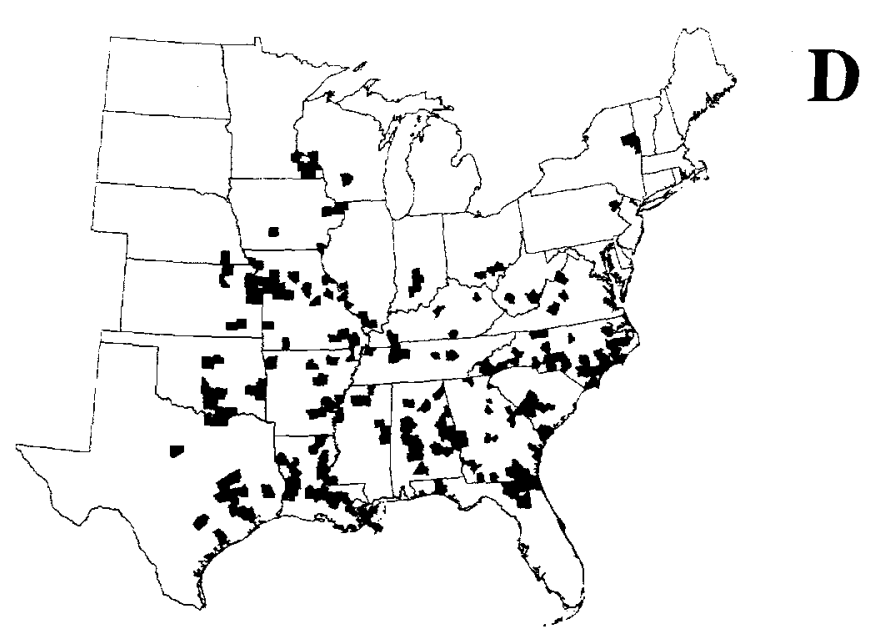

Fig. 15.-Geographic variation in postocular stripe of specimens $>900 \mathrm{~mm}$ SVL. (A) Absent. (B) Light. (C) Partial dark. (D) Solid
dark. Each character state of postocular stripe is mapped bes dark. Each character state of postocular stripe is mapped by county. Descriptions of character states are provided in Appendix II. 

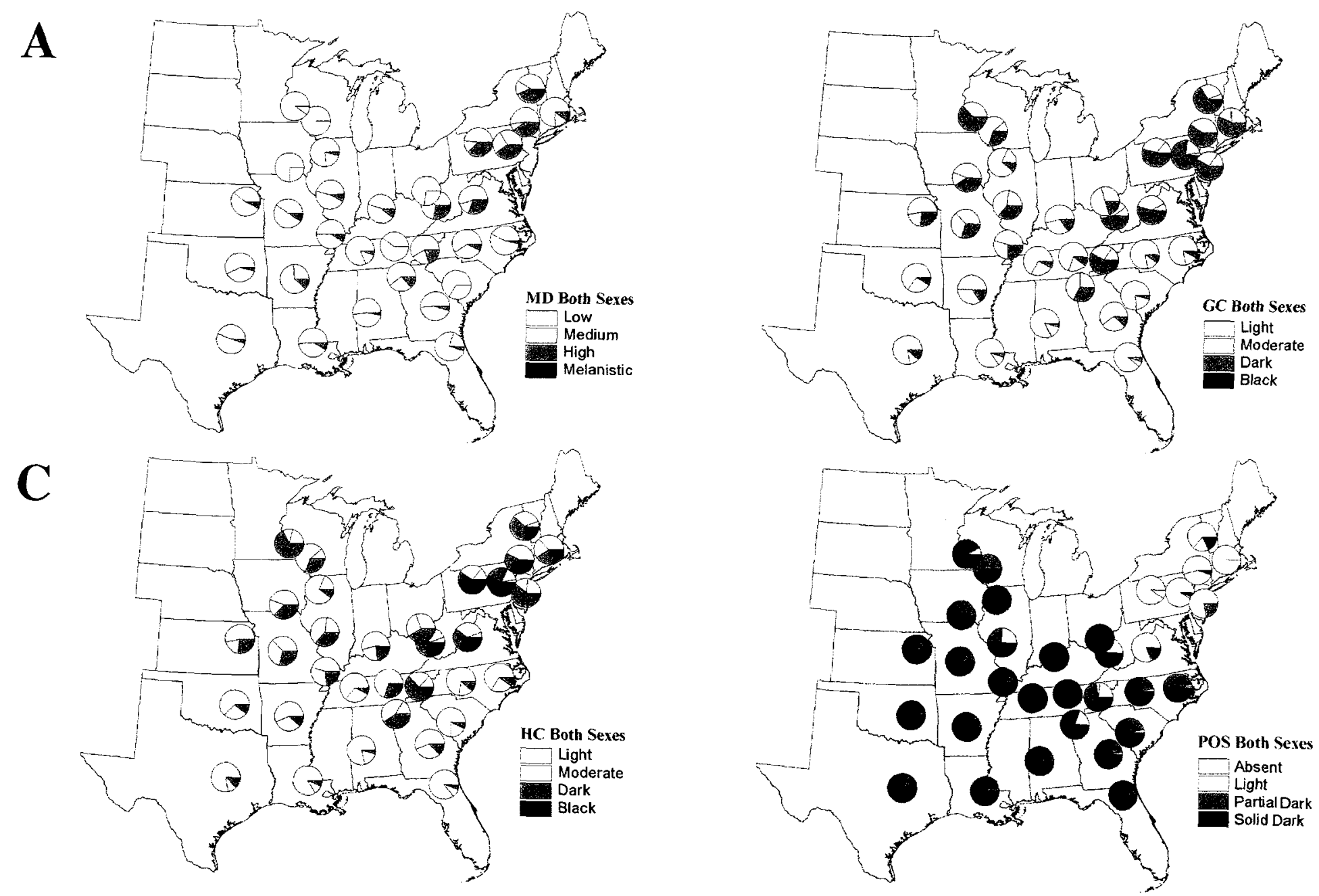

\section{B

$\mathbf{C}$

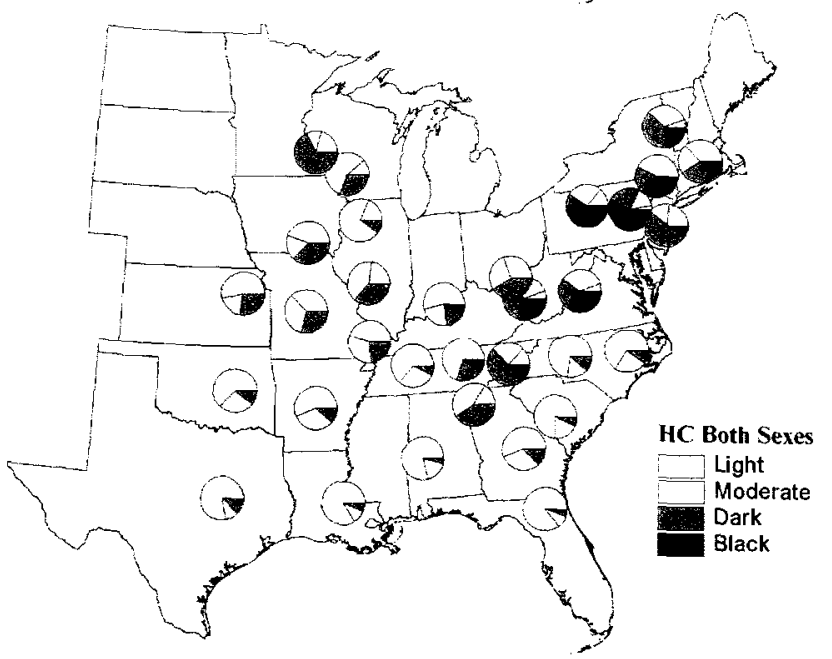

Fig. 16.-Geographic variation in coloration in 32 regions, by frequency. (A) Melanin density. (B) Ground color. (C) Head color. (D) Postocular stripe of specimens $>900 \mathrm{~mm}$ SVL. The character states of each coloration character are mapped as percentages of regional samples. Descriptions of characters are provided in Appendix II. 
the eastern, northwestern, and central-western regions, with scattered individuals in the southwestern and south-central regions. Individuals with light GC were concentrated in the southern regions, with only scattered individuals in the most northern regions. A map of $\mathrm{GC}$ as a percentage of regional populations revealed clinal variation among three general areas (Fig. 16B), similar to those for melanin density. The northeastern and Appalachian regions were characterized by black, dark, and moderate GC, the northwestern regions by dark and moderate $\mathrm{GC}$, and the southern regions by light and moderate GC.

Black head color occurred primarily from the northeastern regions to the southern Appalachians, and was found also in three scattered areas: northern Florida, central Tennessee, and central Kentucky (Fig. 14). Black HC was more common and widespread than black GC and melanistic MD. Dark, moderate, and light states of HC displayed the same general distributional patterns as the respective states of GC. A map of $\mathrm{HC}$ as a percentage of regional populations showed clinal variation among three general areas of differentiation (Fig. 16C), similar to that observed for MD and GC.

Specimens with a solid dark postocular stripe were concentrated in the western and southern regions, but individuals were also scattered throughout the northeastern and north-central regions (Fig. 15). Individuals with a partially dark POS were concentrated in North Carolina and were scattered throughout the western and southern regions. A light POS was observed mostly in specimens from New York. Specimens with POS absent were concentrated in the northeastern regions and the Appalachians. A map of POS as a percentage of regional populations showed clinal variation between two general areas (Fig. 16D). The northeastern regions were characterized by a large proportion of 
individuals with POS absent, whereas the remaining regions generally exhibited a solid dark or partially dark POS. Individuals in the northeastern regions with POS were mostly juveniles and subadults. Thus, because POS is a juvenile feature only in the northeastern regions, its apparent absence in the northeast is misleading and refers to the adult condition only.

\section{Regression Analysis of Meristic Data}

Band length $(\mathrm{BL})$ explained significant variation in band spacing $(\mathrm{BS})$ for males and females (Table 1). Female BS increased with BL at a slightly greater rate than male BS (Fig. 17A). The BL*sex interaction was significant, so the effect of sex alone could not be tested directly. BL also explained significant variation in transverse elements (TE) for males and females. Female TE decreased with $\mathrm{BL}$ at a slightly greater rate than male TE (Fig. 17B), but neither the BL*sex interaction nor the effect of sex was significant.

Band spacing explained significant variation in TE for males and females (Table 1). Male TE decreased with BS at a slightly greater rate than female TE (Fig. 18A), but the BS* sex interaction and the effect of sex were not significant. Transverse elements explained significant variation in body crossbands $(\mathrm{BCB})$ for males and females. Male and female BCB increased very slowly with TE; males had higher BCB and females had higher TE (Fig. 18B). The TE*sex interaction was not significant, but the effect of sex was significant.

Ventral scales (VS) explained significant variation in TE for males and females (Table 1). Although the regressions of TE on VS for males and females were nearly 
Table 1.-ANCOVA of six meristic relationships to test for the effects of sex using the entire data set. Character abbreviations are defined in Appendix II. The following data are reported for each relationship: sex (M or F), regression equation, percentage of variation explained $\left(r^{2}\right)$, sample size $(n), F$-ratio $(F)$, and level of significance $(P)$.

\begin{tabular}{|c|c|c|c|c|c|c|}
\hline Relationship & Sex & Regression Equation & $r^{2}$ & $n$ & $F$ & $P$ \\
\hline \multirow{2}{*}{$\mathrm{BS}$ on $\mathrm{BL}$} & $\mathbf{M}$ & $\mathrm{BS}=5.03+0.480 * \mathrm{BL}$ & 0.189 & 991 & 230.97 & 0.0001 \\
\hline & F & $\mathrm{BS}=4.82+0.580 * \mathrm{BL}$ & 0.257 & 860 & 297.59 & 0.0001 \\
\hline$B L^{*} \operatorname{sex}$ & & & & 1,851 & 4.73 & 0.030 \\
\hline \multirow[t]{2}{*}{$\mathrm{TE}$ on $\mathrm{BL}$} & M & $\mathrm{TE}=31.2-1.55^{*} \mathrm{BL}$ & 0.169 & 1,022 & 208.80 & 0.0001 \\
\hline & $\mathbf{F}$ & $\mathrm{TE}=31.9-1.72 * \mathrm{BL}$ & 0.205 & 884 & 228.74 & 0.0001 \\
\hline $\mathrm{BL}^{*} \mathrm{sex}$ & & & & 1,906 & 1.19 & 0.28 \\
\hline Sex & & & & 1,906 & 1.40 & 0.24 \\
\hline \multirow[t]{2}{*}{ TE on BS } & M & $\mathrm{TE}=40.7-2.17 * \mathrm{BS}$ & 0.401 & 988 & 661.32 & 0.0001 \\
\hline & $\mathrm{F}$ & $\mathrm{TE}=39.7-2.00 * \mathrm{BS}$ & 0.354 & 855 & 468.81 & 0.0001 \\
\hline BS*sex & & & & 1,843 & 1.78 & 0.18 \\
\hline Sex & & & & 1,843 & 3.45 & 0.063 \\
\hline \multirow[t]{2}{*}{$\mathrm{BCB}$ on TE } & M & $\mathrm{BCB}=5.8+0.227 * \mathrm{TE}$ & 0.020 & 1,020 & 21.88 & 0.0001 \\
\hline & $\mathrm{F}$ & $\mathrm{BCB}=4.5+0.236^{*} \mathrm{TE}$ & 0.025 & 883 & 23.72 & 0.0001 \\
\hline TE*sex & & & & 1,903 & 0.02 & 0.90 \\
\hline Sex & & & & 1,903 & 53.70 & 0.0001 \\
\hline \multirow[t]{2}{*}{ TE on VS } & $\mathrm{M}$ & $\mathrm{TE}=-10.5+0.225 * \mathrm{VS}$ & 0.127 & 1,011 & 147.64 & 0.0001 \\
\hline & $\mathrm{F}$ & $\mathrm{TE}=-10.3+0.217 * \mathrm{VS}$ & 0.114 & 877 & 113.46 & 0.0001 \\
\hline VS*sex & & & & 1,888 & 0.08 & 0.78 \\
\hline Sex & & & & 1,888 & 119.89 & 0.0001 \\
\hline \multirow[t]{2}{*}{ CS on VS } & M & $\mathrm{CS}=-2.9+0.173 * \mathrm{VS}$ & 0.123 & 991 & 139.37 & 0.0001 \\
\hline & $\mathrm{F}$ & $\mathrm{CS}=1.2+0.118^{*} \mathrm{VS}$ & 0.068 & 870 & 64.39 & 0.0001 \\
\hline VS*sex & & & & 1,861 & 7.08 & 0.0078 \\
\hline
\end{tabular}



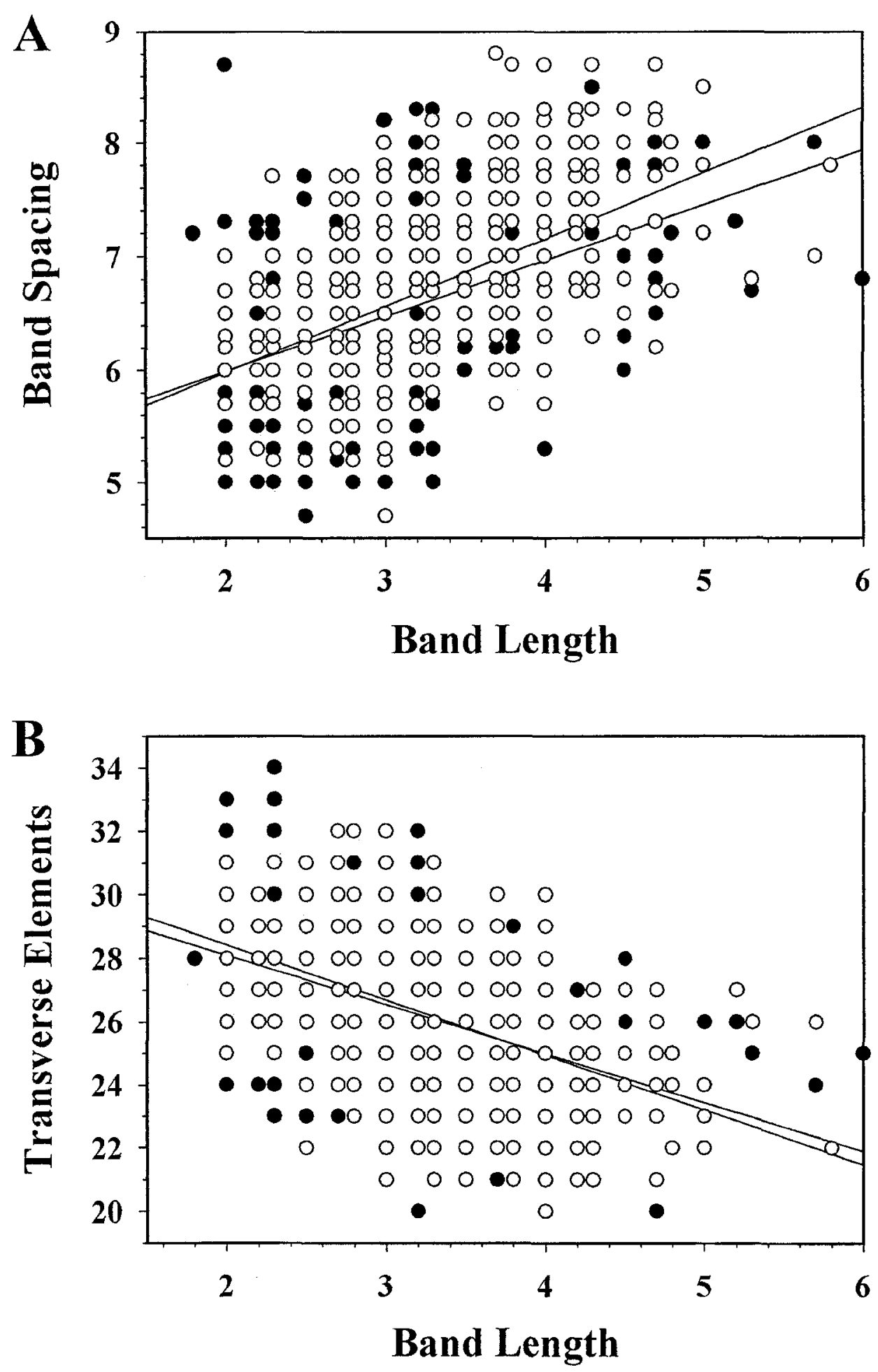

Fig. 17.--Regressions of dorsal pattern elements on band length. (A) Regression of band spacing on band length. (B) Regression of transverse elements on band length. Closed circles $=$ males; open circles $=$ females. 

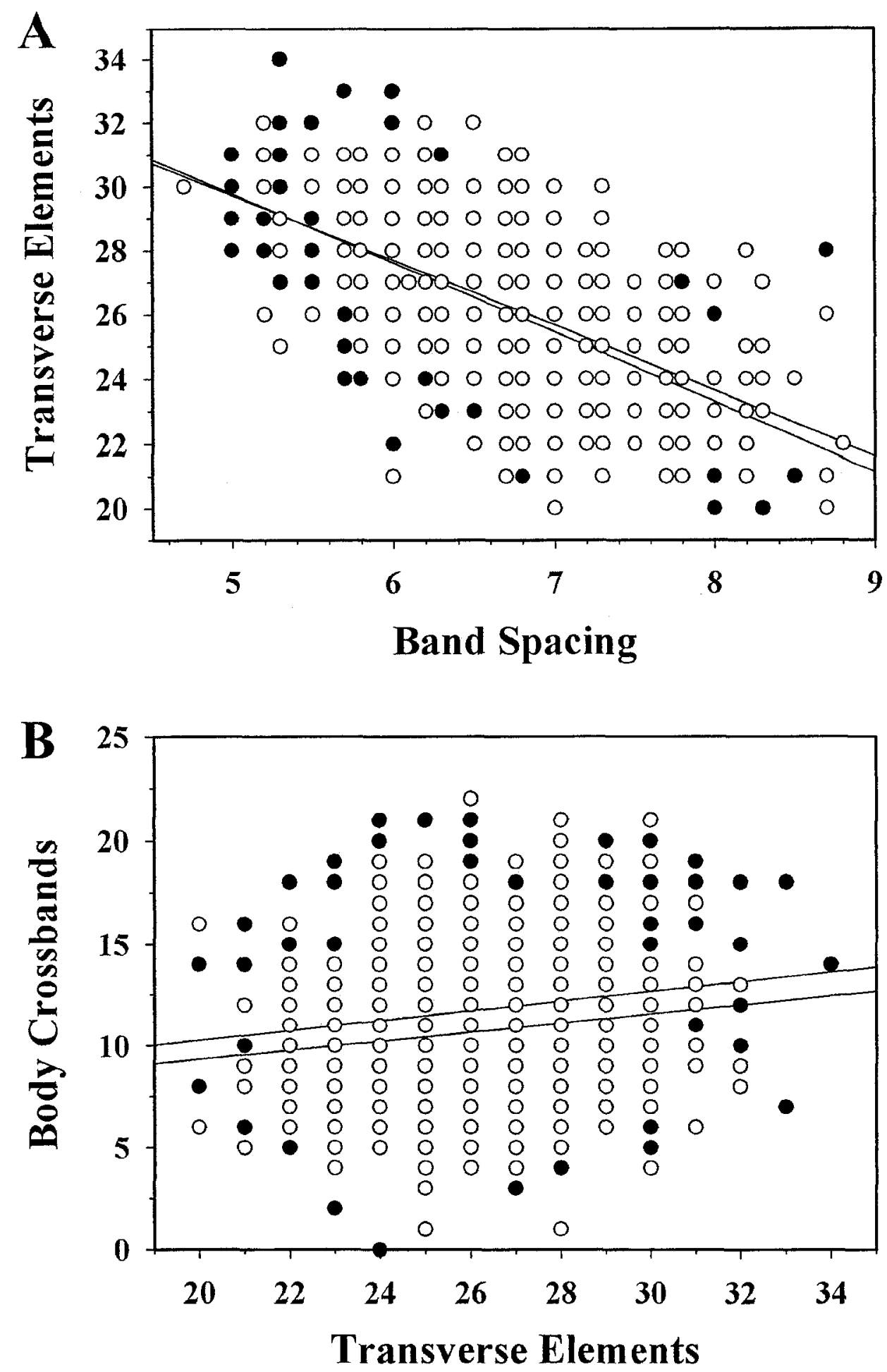

Fig. 18.-Regressions of dorsal pattern elements on band spacing and transverse elements. (A) Regression of transverse elements on band spacing. (B) Regression of body crossbands on transverse elements. Closed circles $=$ males; open circles $=$ females. 


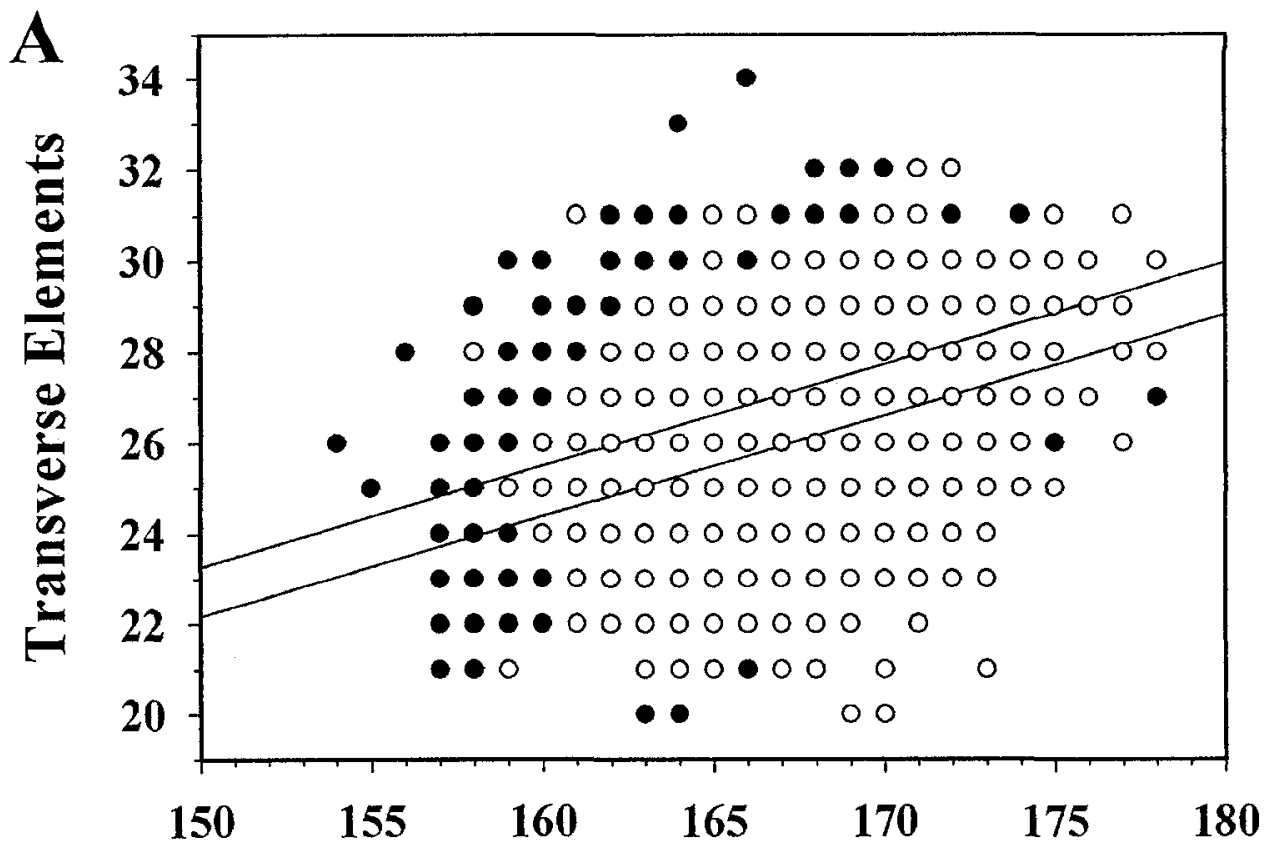

\section{Ventral Scales}

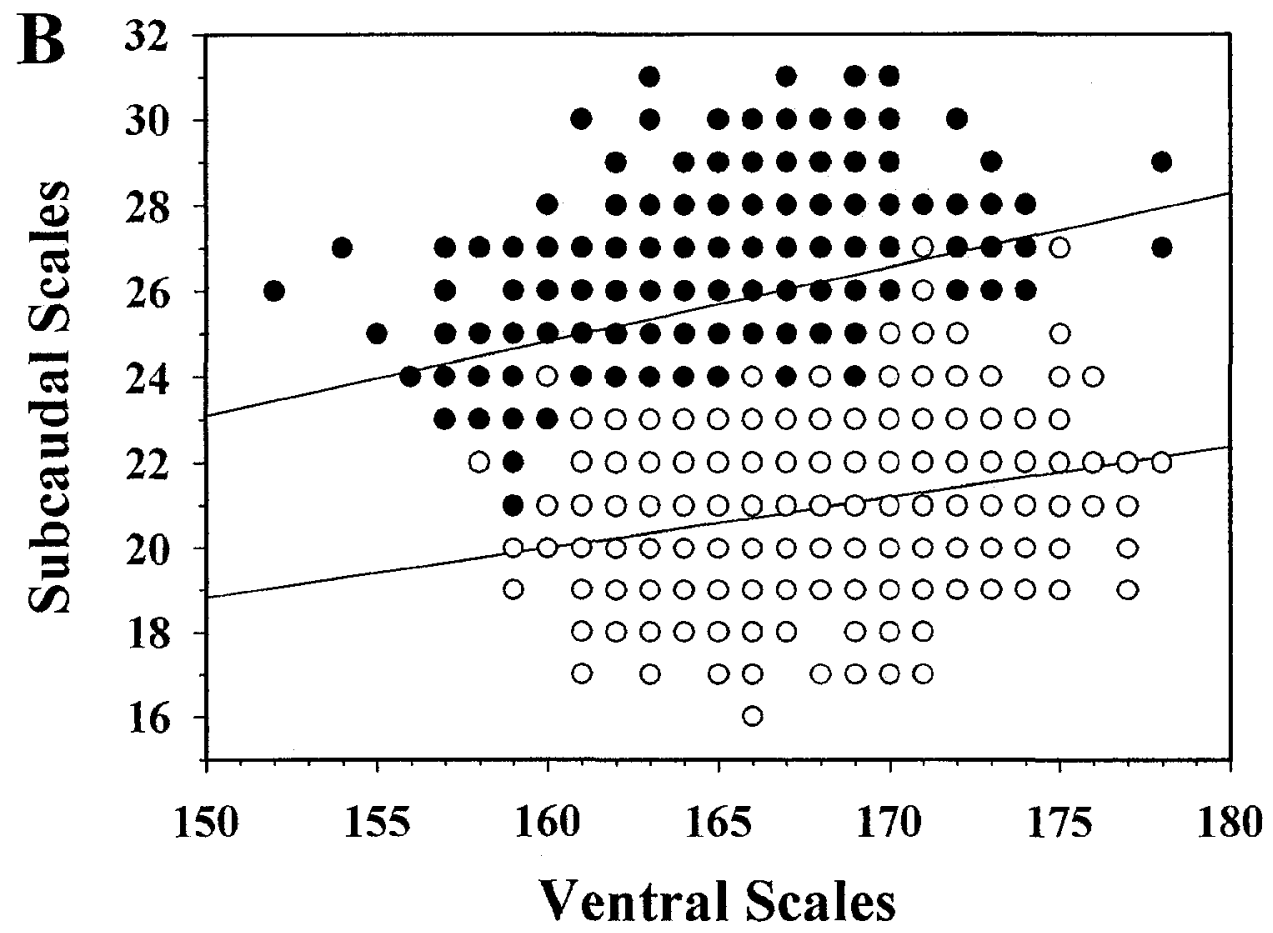

Fig. 19.- Regressions of transverse elements and subcaudal scales on ventral scales. (A) Regression of transverse elements on ventral scales. (B) Regression of subcaudal scales on ventral scales. Closed circles $=$ males; open circles $=$ females. 
parallel, the higher line for males reflects the higher TE of males and higher VS of females (Fig. 19A). The VS* sex interaction was not significant, but the effect of sex was significant. VS also explained significant variation in subcaudal scales (CS) for males and females. Male CS increased with VS at a slightly greater rate than female CS (Fig. 19B). The VS* sex interaction was significant.

\section{Principal Component Analysis of Meristic Data}

Eight variables (DCS, DMB, BCB, HB, UB, YB, TCB, and MSW) were removed from the multivariate analyses because of high skewness, extremely low variances, or nonnormality even after logarithmic transformation. Principal component analysis (PCA) was performed on 16 remaining meristic variables (ADS, MDS, PDS, VS, CS, LSL, RSL, LIL, RIL, LDB, RDB, LVB, RVB, TE, BL, and BS) for males, females, and combined sexes.

Ten principal components (PCs) explained $95 \%$ of the variation for males (Appendix VII, Table 7.1). The most important character contributing to PC1 was band length (BL). The eigenvector structure for each $\mathrm{PC}$ is summarized for $\mathrm{PC} 1$ (BL and BS against LDB, RDB, LVB, RVB, and TE), PC2 (BL, LDB, RDB, LVB, RVB, and TE against a low value for BS), PC3 (LSL, RSL, LIL, and RIL), PC4 (BS and CS), PC5 (LSL and RSL against LIL and RIL), PC6 (CS against BS), PC7 (LDB and LVB against RDB and RVB), PC8 (LSL against RSL), PC9 (ADS and MDS against RIL), and PC10 (LIL against RIL). The absence of two variables that were prominent in previous studies was a 
striking result. Midbody dorsal scale number (MDS) did not load substantially until PC11 and ventral scale number (VS) did not do so until PC16.

Ten PCs also explained $95 \%$ of the variation for females (Appendix VII, Table 7.2). As in males, the most important character in PC1 was BL. The eigenvector structure for each PC is summarized for PC1 (BL and BS against LDB, RDB, LVB, RVB, and TE), PC2 (BL, LDB, RDB, LVB, RVB, and TE), PC3 (LSL, RSL, LIL, and RIL against BL), PC4 (BS and CS), PC5 (CS against LSL and RSL), PC6 (LSL and CS against LIL and RIL), PC7 (ADS, MDS, LDB, and LVB against RDB, RVB, and RIL), PC8 (ADS and MDS against LSL and LVB), PC9 (RSL and LIL against LSL and RIL), and PC10 (LIL and LSL against RIL and RSL). Again, MDS did not load substantially until PC11 and VS did not do so until PC16.

Similarly, ten principal components (PCs) explained $95 \%$ of the variation for combined sexes (Appendix VII, Table 7.3). Again, the most important character in PC1 was $\mathrm{BL}$. The eigenvector structure for each PC is summarized for PC1 (BL and BS against LDB, RDB, LVB, RVB, and TE), PC2 (BL, LDB, RDB, LVB, RVB, and TE), PC3 (CS), PC4 (LSL, RSL, LIL, and RIL), PC5 (BS), PC6 (BS, LDB, RDB, LVB, and RVB), PC7 (LDB and LVB against RDB and RVB), PC8 (ADS and MDS), PC9 (LSL against RSL), and PC10 (LIL against RIL). MDS again did not load substantially until PC11 and VS not until PC16.

In summary, PCAs of males, females, and combined sexes were very similar in structure. Band length (BL) was the most important variable in all three analyses. The primary effect of analyzing males and females separately resulted in a shift of subcaudal scales (CS) from PC3 in the analysis of the combined sexes to PC4 and PC6 in males and 
to PC4 and PC5 in females. The magnitude of the loadings of CS greatly decreased in the separate analyses of males and females, as would be expected.

\section{Cluster Analysis of Meristic Data}

Ward's minimum-variance cluster analysis produced distinct but geographically overlapping clusters for males, females, and combined sexes. Concensus of the diagnostic statistics indicated that five clusters were appropriate in all three models. For each of the following diagnostic statistics, the number of clusters identified by that test are shown in parentheses for males: CCC (3), PSTSQ (4 and 7), PSF (8), SPRSQ (4 and 6), RSQ (3 and 7), BSS (5), and RMSSTD (3 and 6); for females: CCC (4), PSTSQ (3 and 6), PSF (7), SPRSQ (3 and 6), RSQ (6), BSS (4 and 6), and RMSSTD (2 and 6); and for combined sexes: CCC (3 and 5), PSTSQ (2 and 5), PSF (6 and 9), SPRSQ (5 and 7), RSQ (5 and 7), BSS (5 and 8), and RMSSTD (4 and 7). Analyzing each sex separately did not reduce the number of clusters compared to the combined-sexes model. Dendrograms helped visualize the clustering procedure and were plotted against BSS for males, females, and combined sexes (Fig. 20). All three dendrograms supported cluster solutions between three and eight clusters; in that region levels of BSS began to increase sharply, indicating the presence of a cluster solution.

Although a consensus of the diagnostic statistics indicated a preferred solution of five clusters, analyses were performed independently on two-, three-, four-, and fivecluster models to ensure a complete analysis of the clustering structure. The basic structure for each cluster analysis was summarized for males (Appendix VIII, Table 8.1), 

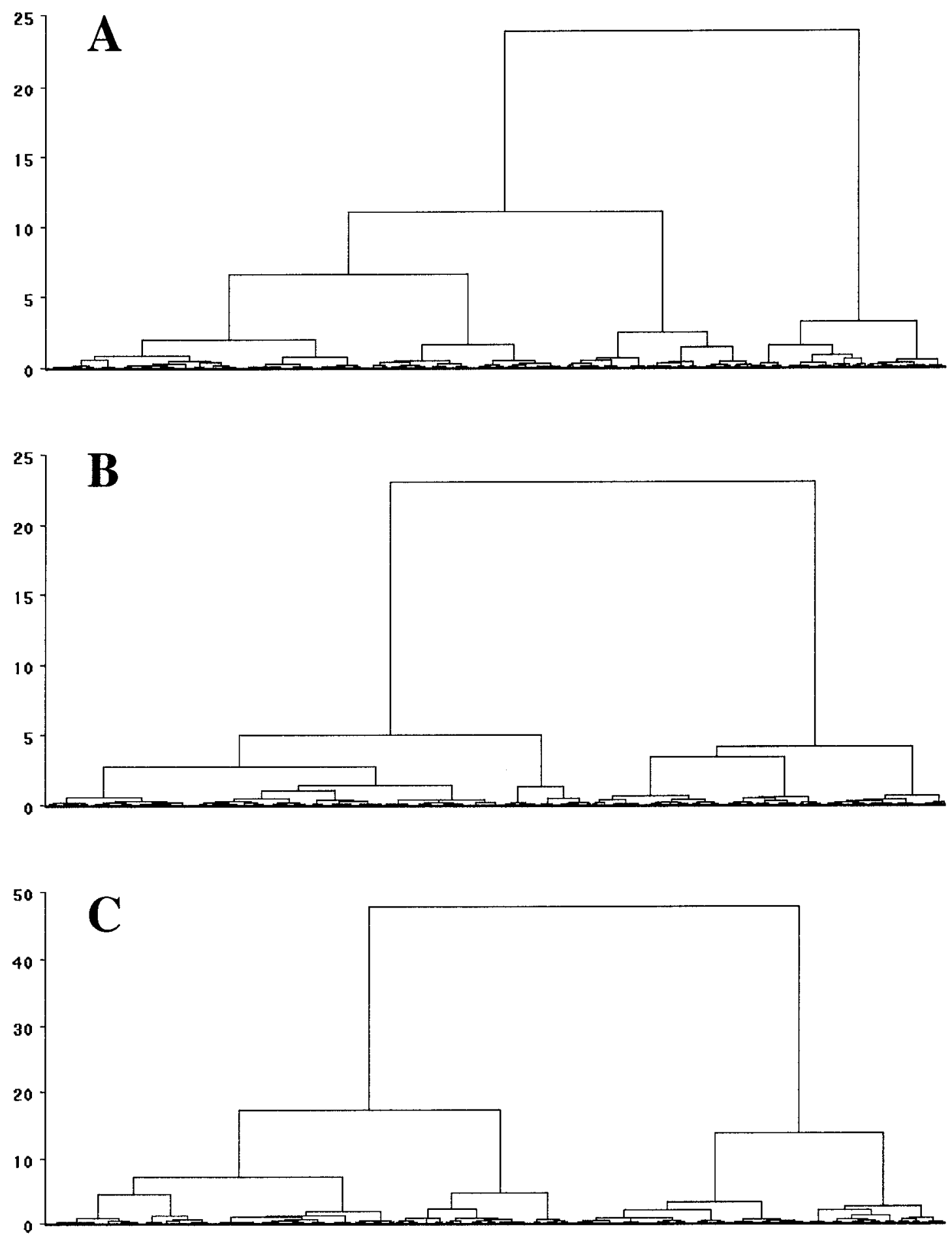

Fig. 20.-Dendrograms for cluster analyses using Ward's minimum-variance method. (A) Males. (B) Females. (C) Combined sexes. Individual observations are arranged on the horizontal axis (resolution is limited due to the scale of the figure) and are grouped hierarchically into larger clusters. The between-group sum of squares is indicated on the vertical axis. 
females (Appendix VIII, Table 8.2), and combined sexes (Appendix VIII, Table 8.3). Similarly, the canonical discriminant analysis was summarized for males (Appendix IX, Table 9.1), females (Appendix IX, Table 9.2), and combined sexes (Appendix IX, Table 9.3).

The canonical variables were plotted to show the clusters in morphological space for males, females, and combined sexes, but only the plots for combined sexes are presented here. The two-cluster model only had one canonical variable, so it was not plotted. The three-cluster (Fig. 21A), four-cluster (Fig. 22A), and five-cluster (Fig. 22B) models for combined sexes all revealed well-defined clusters. Although the clusters within each model had overlapping edges and little overall separation, each cluster exhibited a distinct central area in multivariate morphospace. Canonical discriminant analysis was also performed on Martin's regional morphs for combined sexes (Fig. 21B). The regional morphs exhibited greater overlap than did the results of the cluster analyses. In fact, the southern and western morphs overlapped extensively, and the northern and western morphs overlapped moderately. Separate analyses of males and females, not shown here, produced similar results.

\section{Discriminant Function Analysis of Meristic Data}

Discriminant function analysis (DFA) validated the classifications produced by the cluster analysis (Appendix X, Tables 10.1-10.18). The two-cluster models had the lowest crossvalidation error rates, at $4.5 \%$ for males (Table 10.1 ), 5.1\% for females (Table 10.2), and 7.8\% for combined sexes (Table 10.3). The crossvalidation error rates 

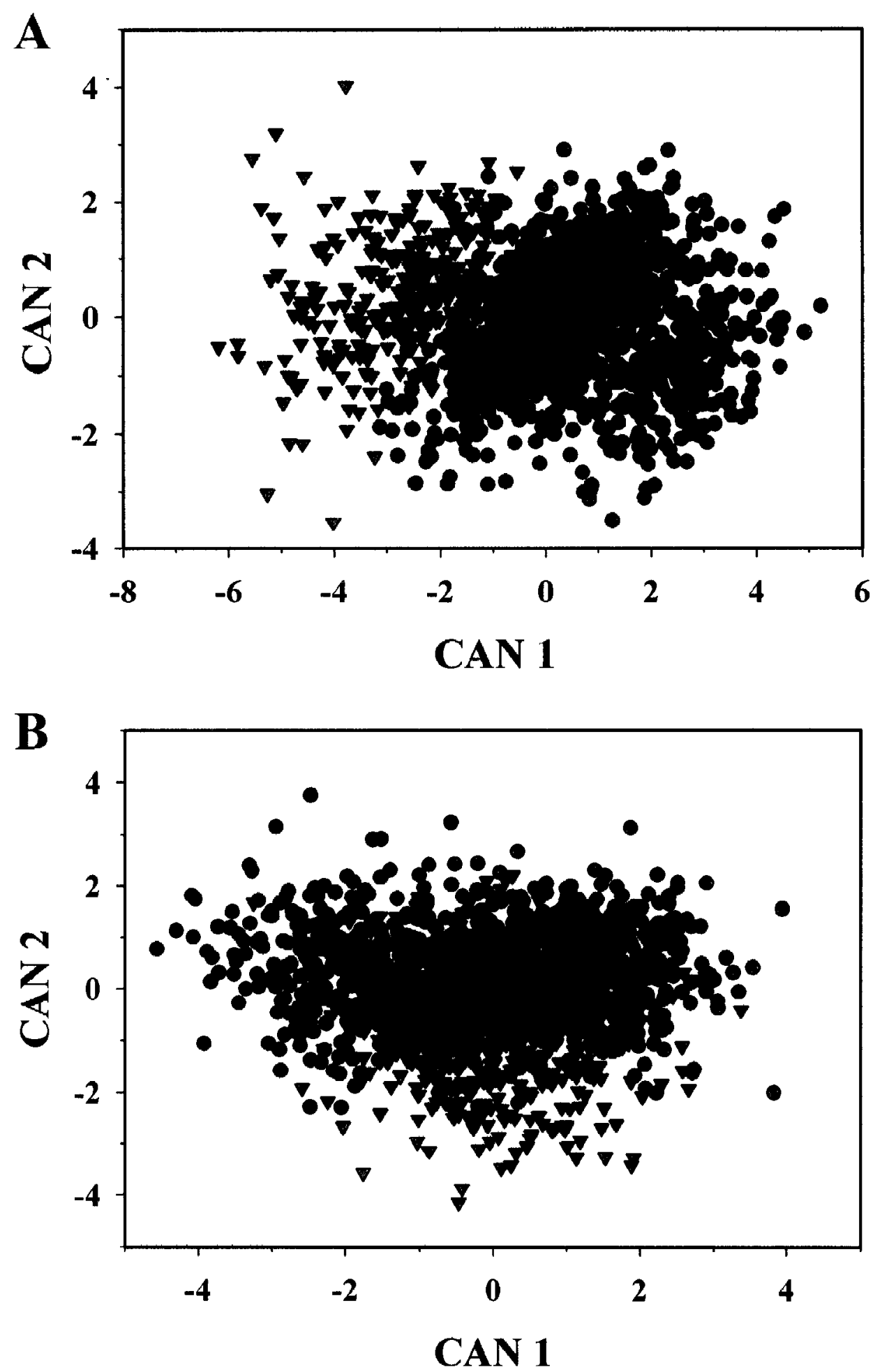

Fig. 21.-...Plot of first two canonical variables of three-cluster models for combined sexes. (A) Three-cluster model. Black circles $=$ Cluster 1 ; red circles $=$ Cluster 2 ; green triangles $=$ Cluster 3. (B) Martin's regional morphs. Black circles = northern morphs; red circles $=$ southern morphs; green triangles $=$ western morphs. 


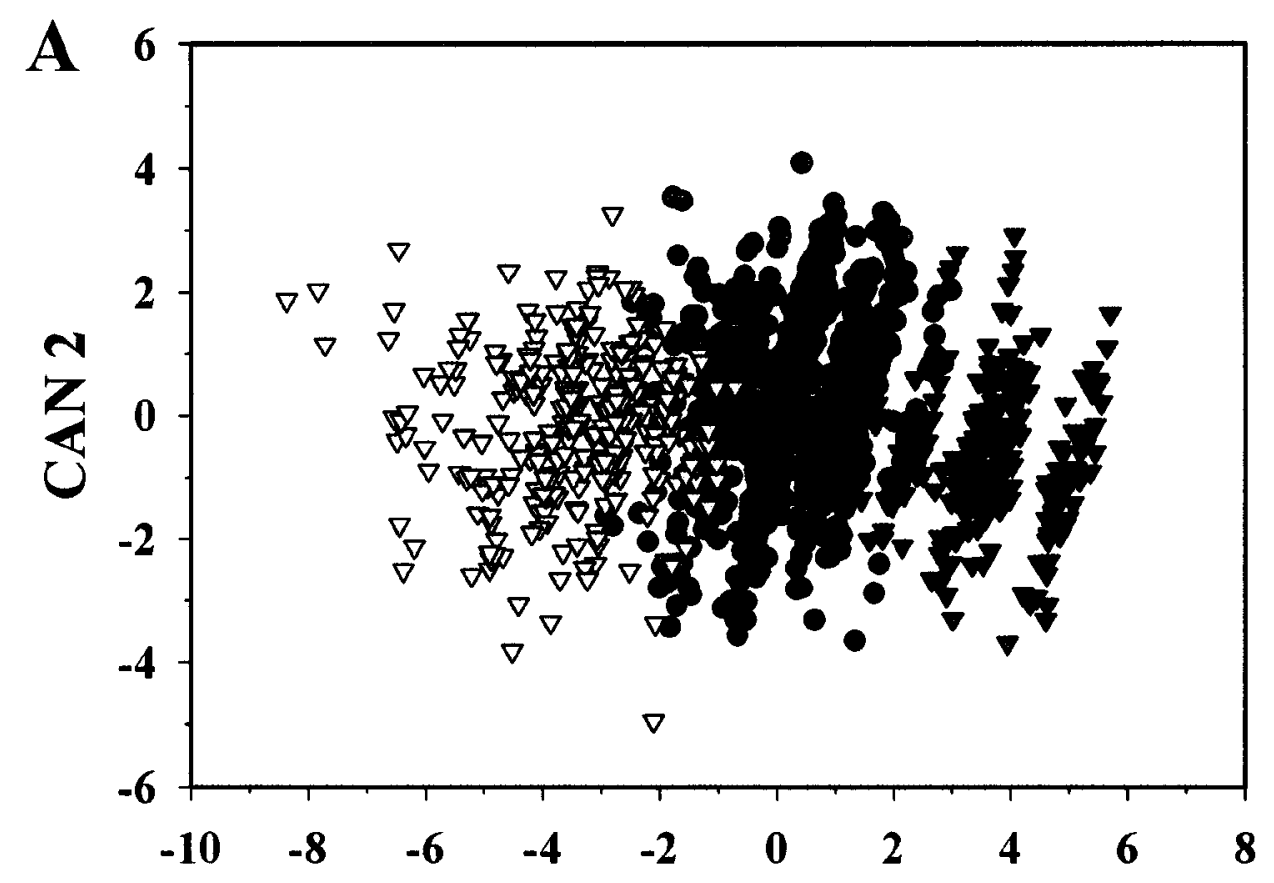

\section{CAN 1}

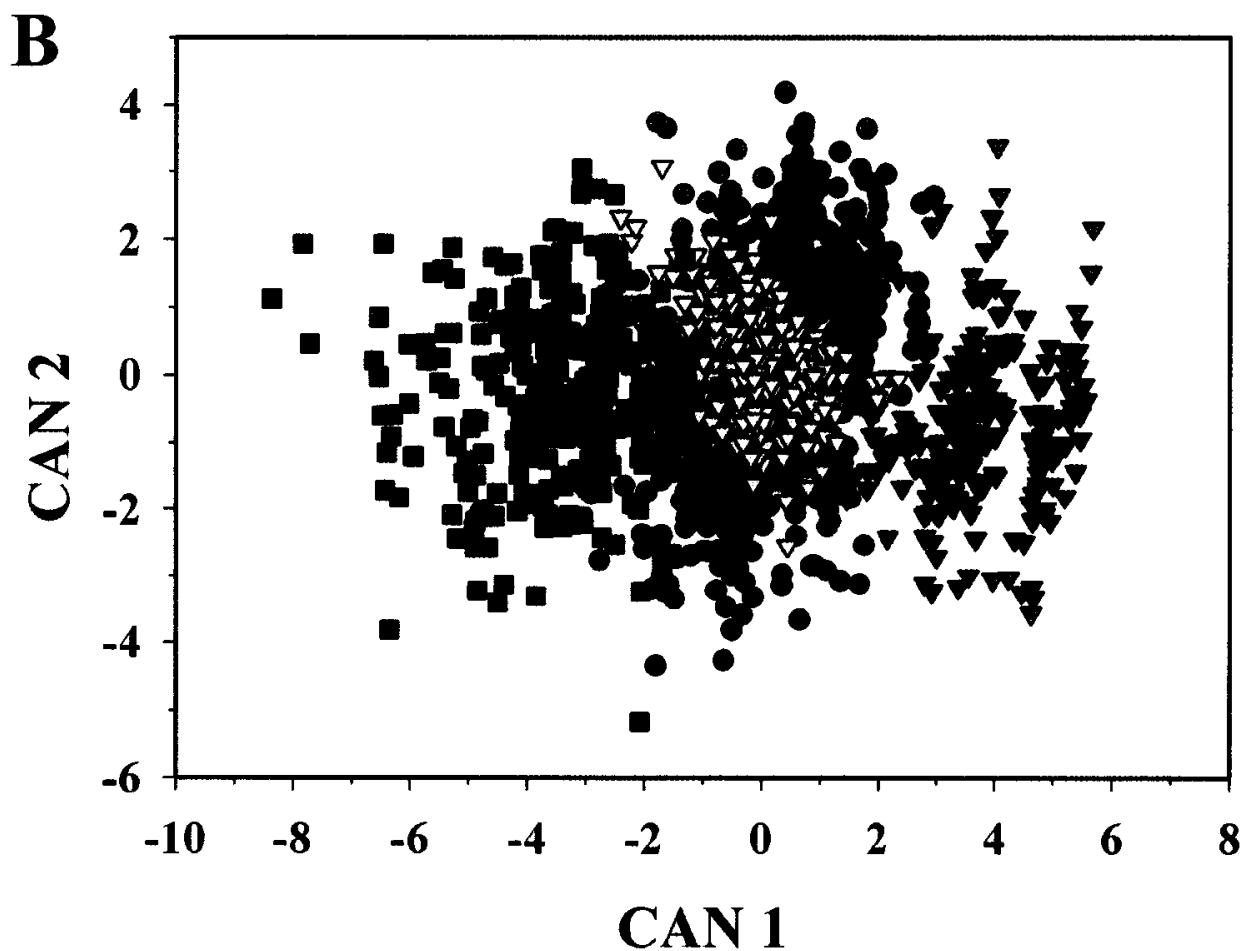

Fig. 22.-Plots of first two canonical variables of four-cluster and five-cluster models for combined sexes. (A) Four-cluster model. (B) Five-cluster model. Black circles $=$ Cluster 1; red circles $=$ Cluster 2; green triangles $=$ Cluster 3 ; yellow triangles $=$ Cluster 4 ; blue squares $=$ Cluster 5 . 
for the three-cluster models were $6.9 \%$ for males (Table 10.4 ), $7.5 \%$ for females (Table 10.5), and $10.8 \%$ for combined sexes (Table 10.6). The crossvalidation error rates for the four-cluster models were $10.0 \%$ for males (Table 10.7), 7.2\% for females (Table 10.8), and $9.6 \%$ for combined sexes (Table 10.9). The crossvalidation error rates for the fivecluster models were $10.8 \%$ for males (Table 10.10), 9.7\% for females (Table 10.11), and $10.4 \%$ for combined sexes (Table 10.12).

The DFA of Gloyd's subspecies did not explain the variation in the data as well as any of the models created by the cluster analysis. The crossvalidation error rates for Gloyd's subspecies were $22.2 \%$ for males (Table 10.13), $23.2 \%$ for females (Table 10.14), and $22.6 \%$ for combined sexes (Table 10.15). The DFA of Martin's regional morphs found even weaker support, due to high numbers of misclassifications. The crossvalidation error rates for Martin's regional morphs were $31.6 \%$ for males (Table 10.16), 32.6\% for females (Table 10.17), and 30.9\% for combined sexes (Table 10.18). Thus, although Gloyd's and Martin's classifications exhibit geographic cohesiveness, the identified classes do not conform closely to groups of specimens that are homogeneous in multivariate morphospace.

\section{Geographic Analysis of Multivariate Clusters}

Although neither the Gloyd nor the Martin classification was strongly supported, the clusters generated by each multivariate cluster analysis revealed a geographic component to the morphological variation found in Crotalus horridus. The northeastern regions remained moderately distinct in all cluster analyses and was best defined in the 
models of males and females analyzed separately. The northwestern regions appeared very distinct in the three-, four-, and five-cluster models for females. However, in all models the clusters showed extensive geographic overlap. Separate analyses of males and females generally improved the separation among clusters and moderately improved geographic cohesiveness in comparison to the models for combined sexes.

The two-cluster models (Figs. 23-26) produced two widespread clusters for males (Fig. 23). Individuals belonging to Cluster 2 (CL2) were concentrated in the northeastern and central-eastern regions, whereas individuals in Cluster 1 (CL1) were spread across the entire range, although these occurred mostly in the western, southern, and extreme northeastern regions. Most overlap between individuals in CL1 and CL2 occurred in the northeastern and central-eastern regions. A map of clusters as a percentage of regional populations revealed a pattern of strong clinal variation from the northeastern regions, dominated by CL2, to the northwestern, southwestern, and southeastern regions, dominated by CL1 (Fig. 26A).

The two-cluster model for females produced two widespread clusters similar to those for males (Fig. 24). The amount of overlap between individuals in CL1 and CL2 was considerably less for females in the northeastern regions, but there was moderate overlap in the central-eastern and central-western regions. A map of clusters as a percentage of regional populations revealed the same pattern of clinal variation as for males, but individuals in the northeastern regions mostly belonged to CL1 (Fig. 26B).

The two-cluster model for combined sexes produced two widespread clusters that differed from those of males and females (Fig. 25). Individuals belonging to CL1 were spread across the entire range, but were mostly concentrated in the northeastern, central- 

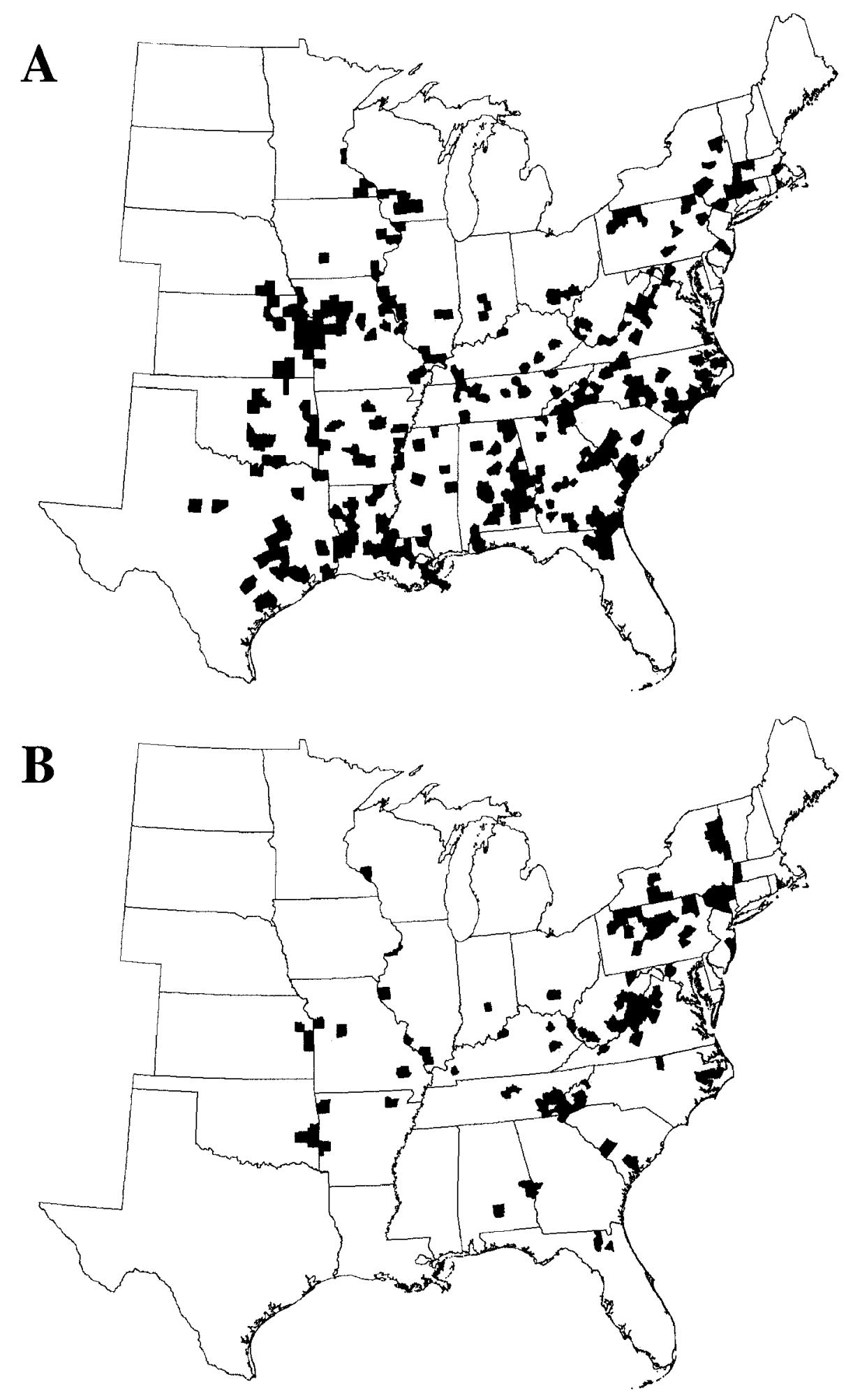

Fig. 23.-Maps of geographic clusters of the two-cluster model for males. (A) Cluster 1. (B) Cluster 2. Counties are plotted if they contain at least one specimen classified in the respective cluster. 

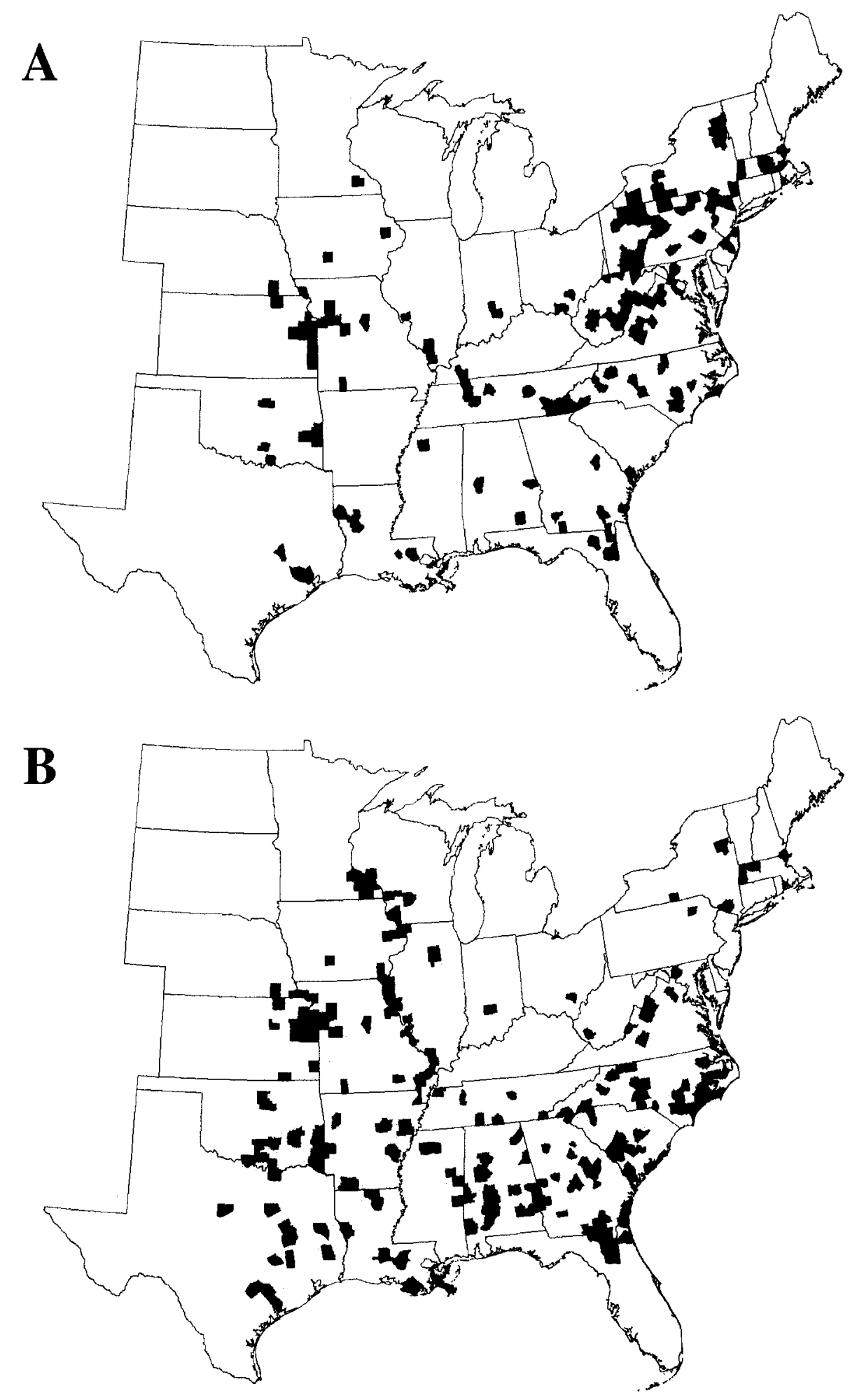

Fig. 24.- Maps of geographic clusters of the two-cluster model for females. (A) Cluster 1. (B) Cluster 2. Counties are plotted if they contain at least one specimen classified in the respective cluster. 

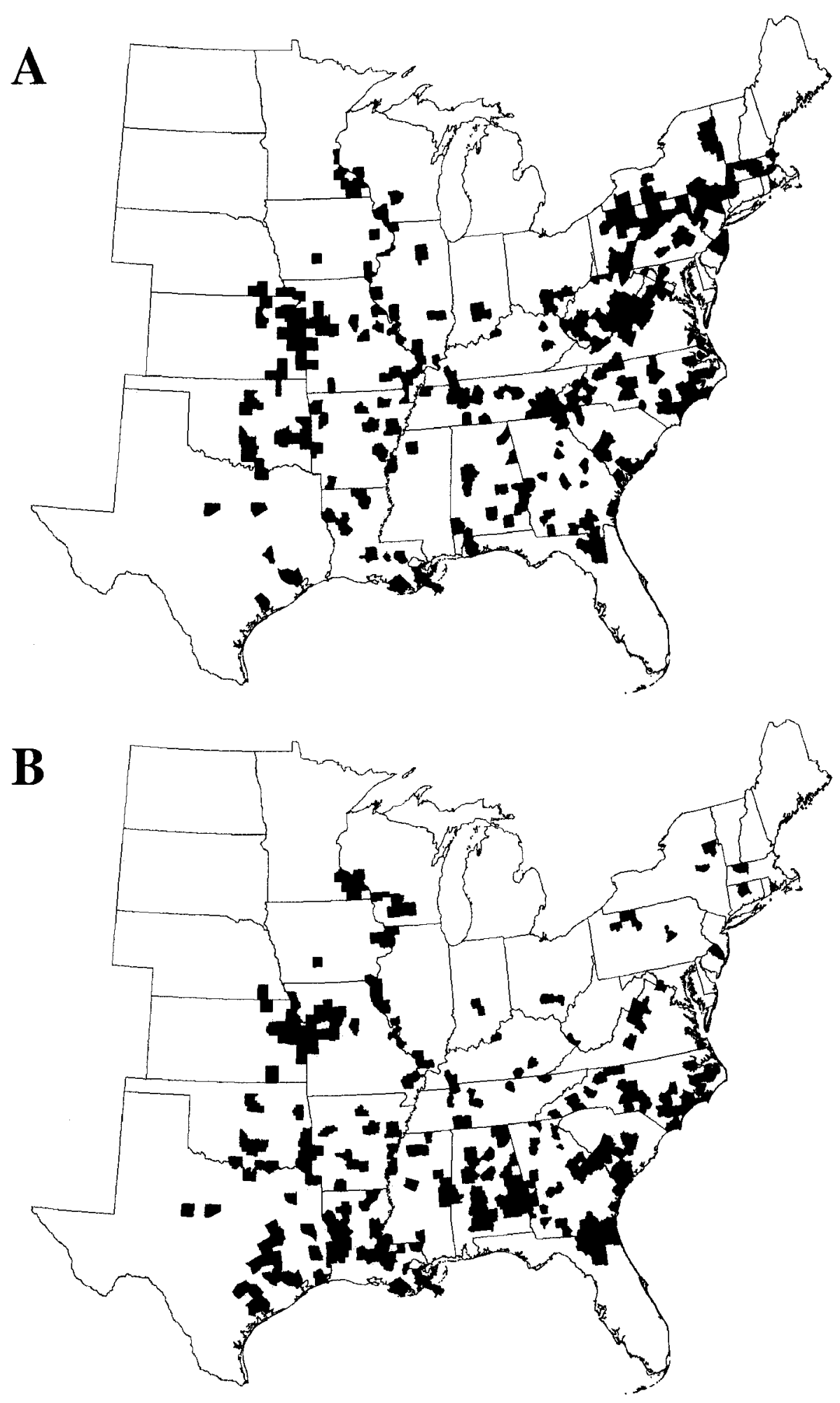

Fig. 25.-Maps of geographic clusters of the two-cluster model for combined sexes. (A) Cluster 1. (B) Cluster 2. Counties are plotted if they contain at least one specimen classified in the respective cluster. 


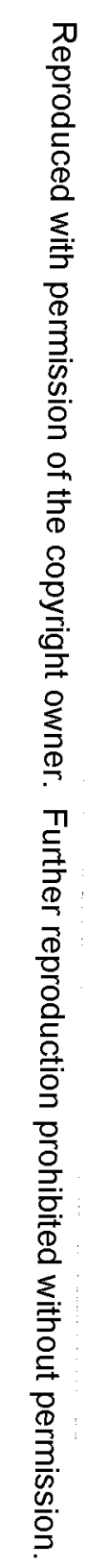

A
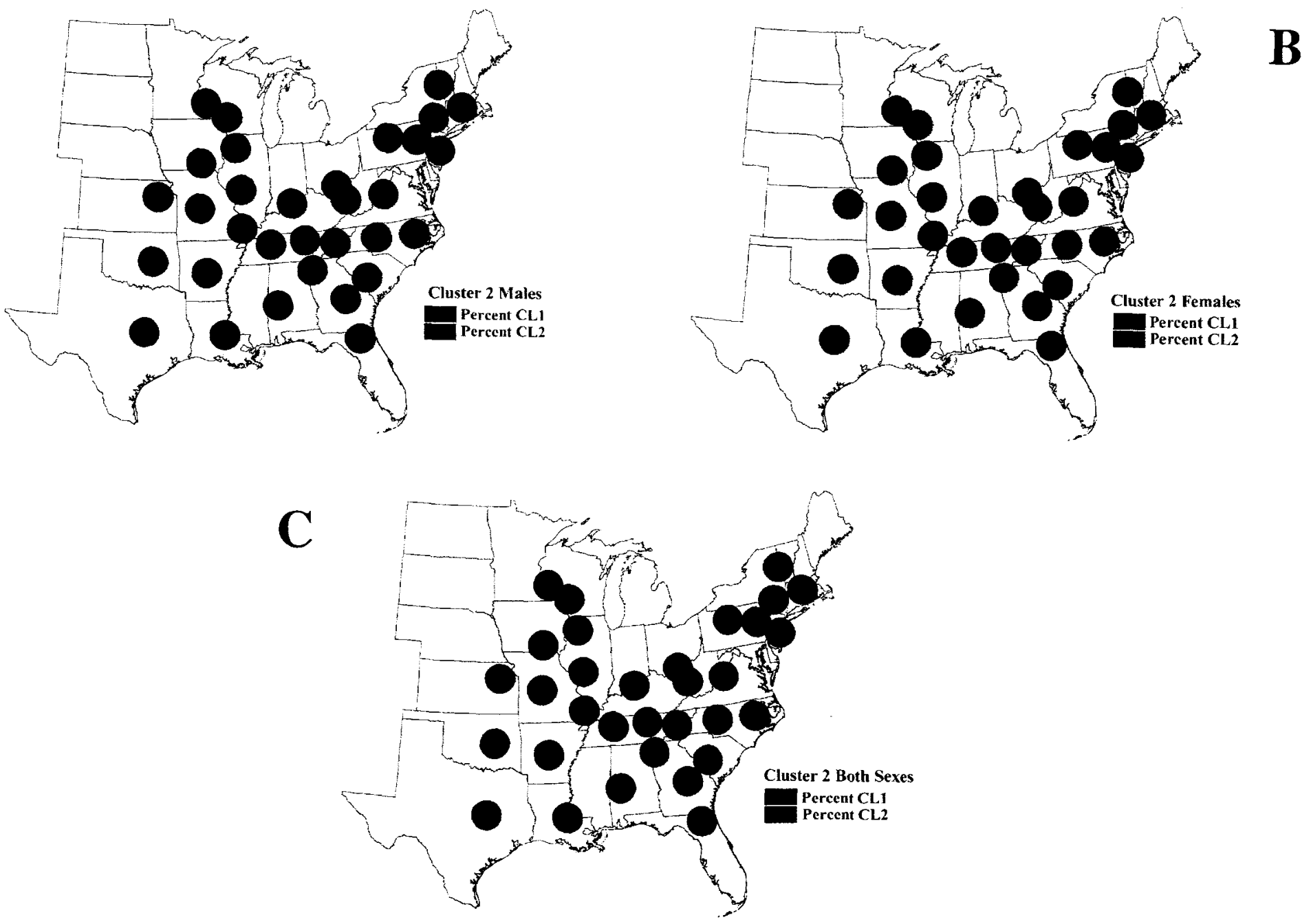

Fig. 26.-Maps of geographic clusters as percentages of regional populations for the two-cluster models. (A) Males. (B) Females. (C) Combined sexes. Each pie chart corresponds to one of the 32 regions shown in Figures 3 and 4. 
eastern, and central-western regions, whereas individuals in CL2 were widespread in the western and southern regions. The amount of overlap between individuals in CL1 and CL2 was considerable throughout the western and southern regions. A map of clusters as a percentage of regional populations revealed the same pattern of clinal variation as for males and females analyzed separately (Fig. 26C).

The three-cluster models (Figs. 27-30) produced three widespread clusters for males (Fig. 27). Individuals belonging to CL2 and CL3 exhibited distributions similar to those in the two-cluster models for males, but shared even less overlap. Individuals in CL1 were spread across the entire range and were overlapped extensively with those in CL2 and CL3. A map of clusters as a percentage of regional populations revealed a pattern of strong clinal variation from the northeastern regions, dominated by CL3, to the southeastern regions, dominated by CL1, and a pattern of weaker clinal variation from the northwestern regions, dominated by CL2, to the southwestern regions, represented by CL1 and CL2 (Fig. 30A). High percentages of CL1 were conspicuous in the extreme northeastern regions and were similar to disjunct values for some individual characters.

The three-cluster model for females produced two widespread clusters and one cluster with a limited distribution (Fig. 28). Individuals belonging to CL1 and CL2 exhibited similar distributions to those for males, whereas individuals in CL3 were mostly limited to the northwestern and central-western regions. A map of clusters as a percentage of regional populations revealed two patterns of strong clinal variation (Fig. 30B). One cline extended from the northeastern regions, dominated by CL1, to the southeastern regions, dominated by CL2. Another cline extended from the northwestern regions, dominated by CL3, to the southwestern regions, dominated by CL2. 

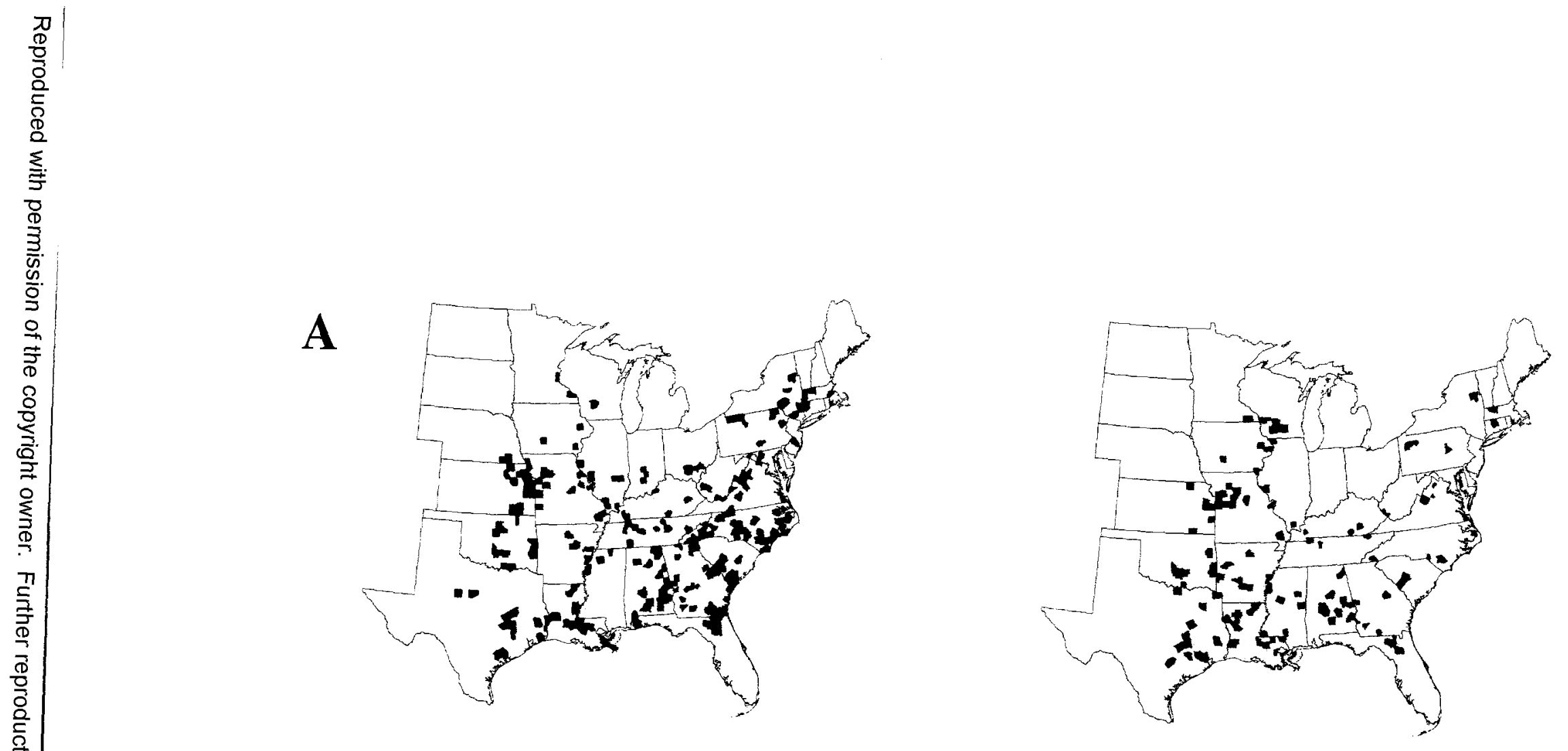

\section{B}

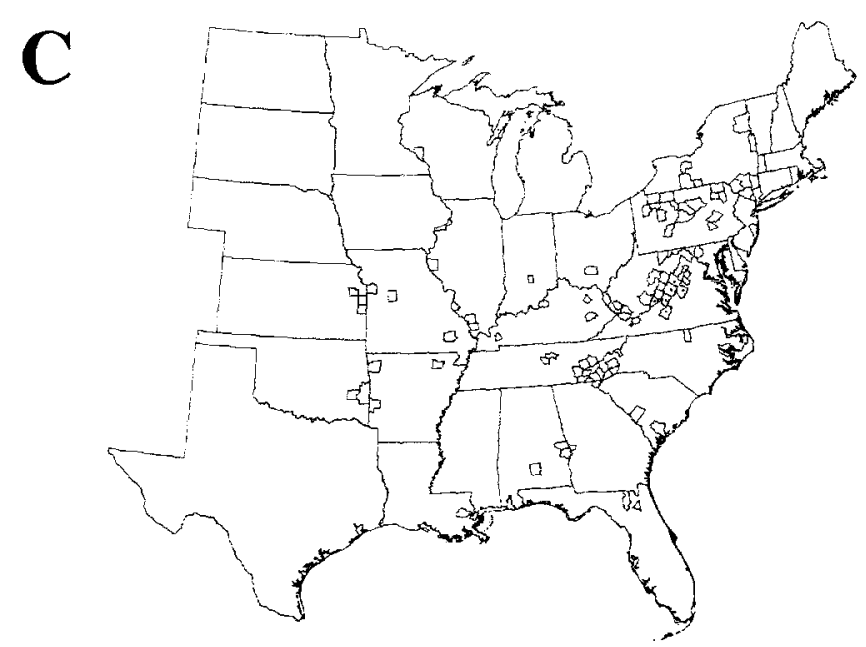
Fig. 27.-Maps of geographic clusters of the three-cluster model for males. (A) Cluster 1. (B) Cluster 2. (C) Cluster 3 . Counties are
plotted if they contain at least one specimen classified in the respective cluster. 

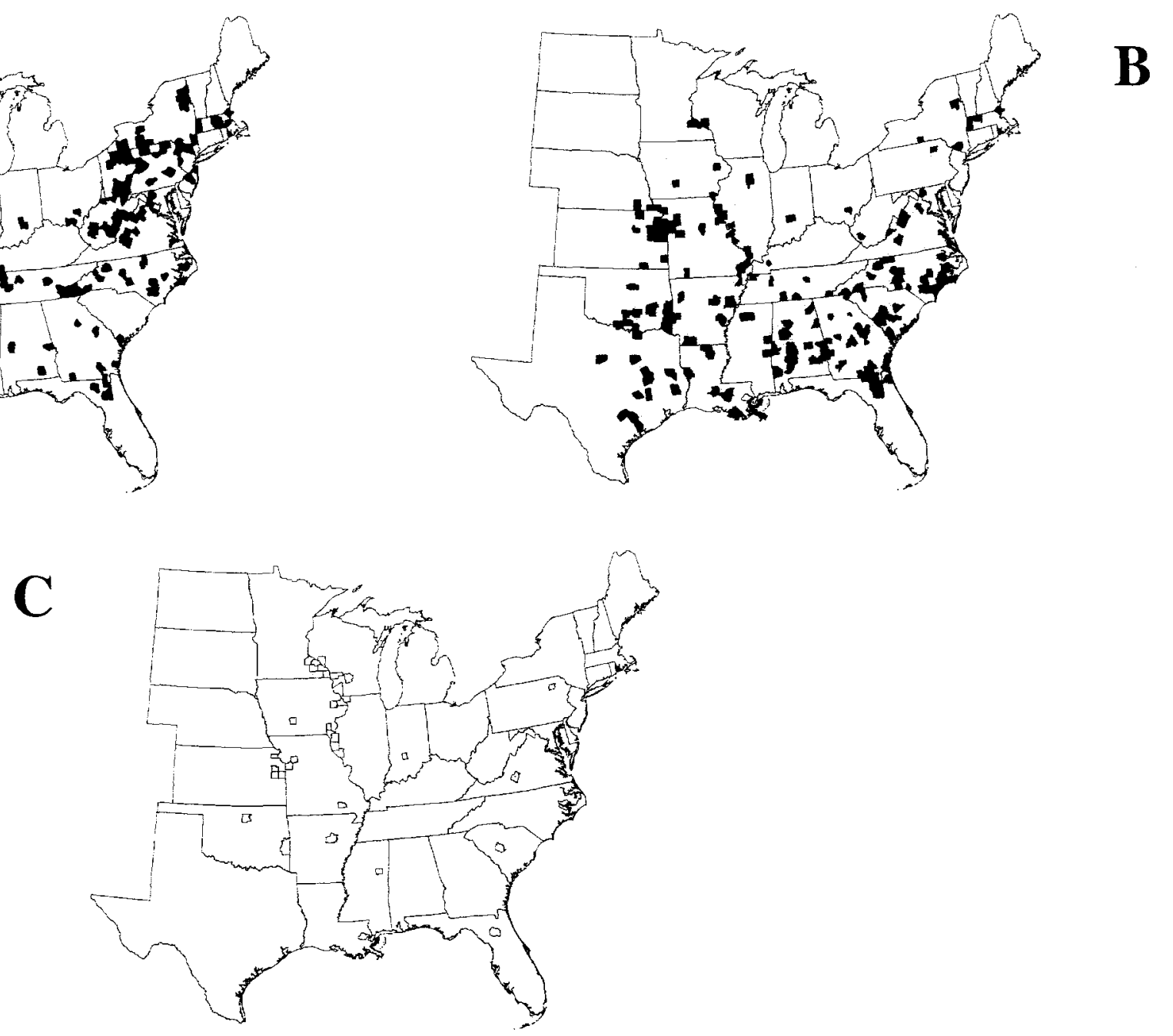

Fig. 28.-Maps of geographic clusters of the three-cluster model for females. (A) Cluster 1. (B) Cluster 2. (C) Cluster 3. Counties are plotted if they contain at least one specimen classified in the respective cluster. 
A
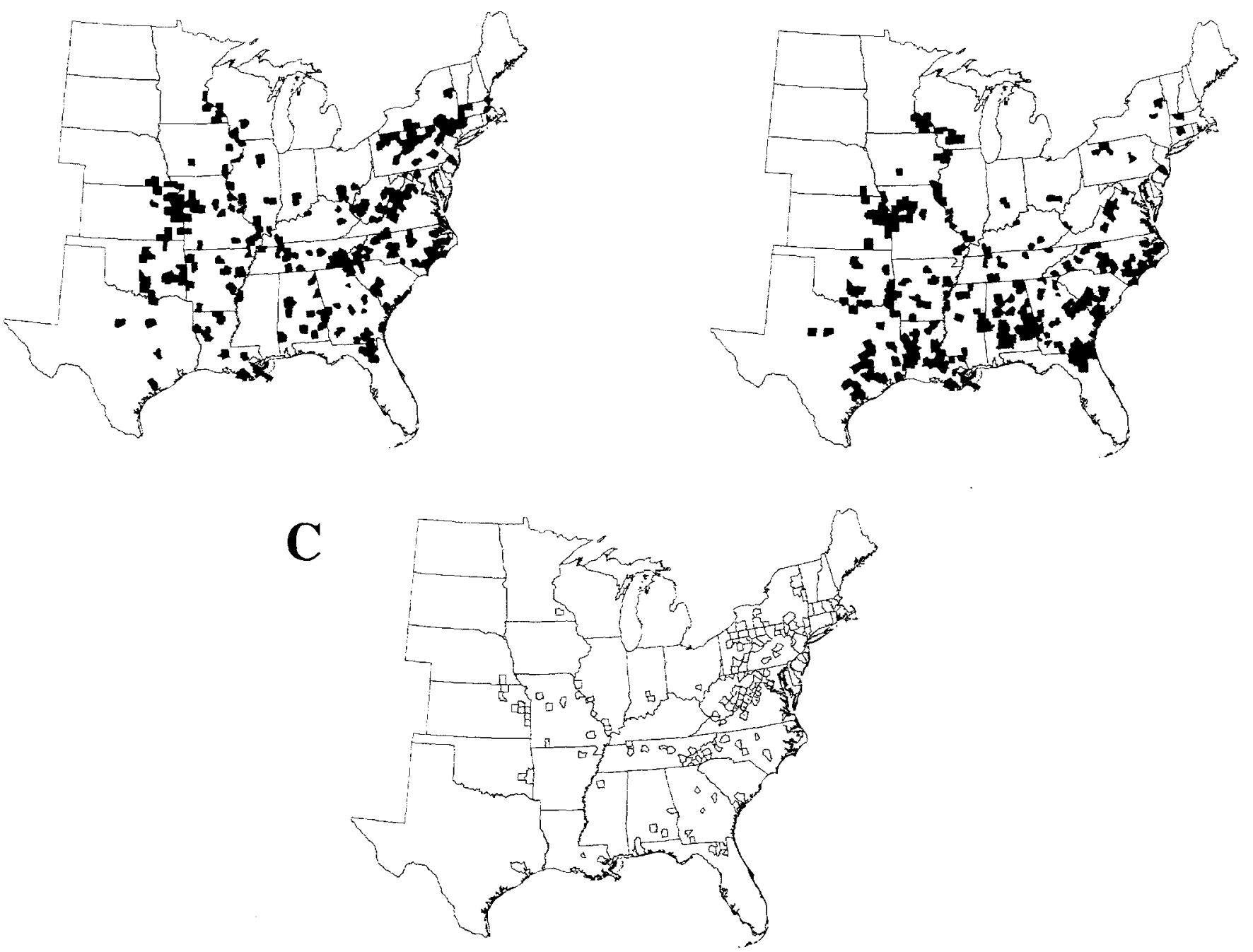

Fig. 29.-Maps of geographic clusters of the three-cluster model for combined sexes. (A) Cluster 1. (B) Cluster 2 . (C) Cluster 3. Counties are plotted if they contain at least one specimen classified in the respective cluster. 
A
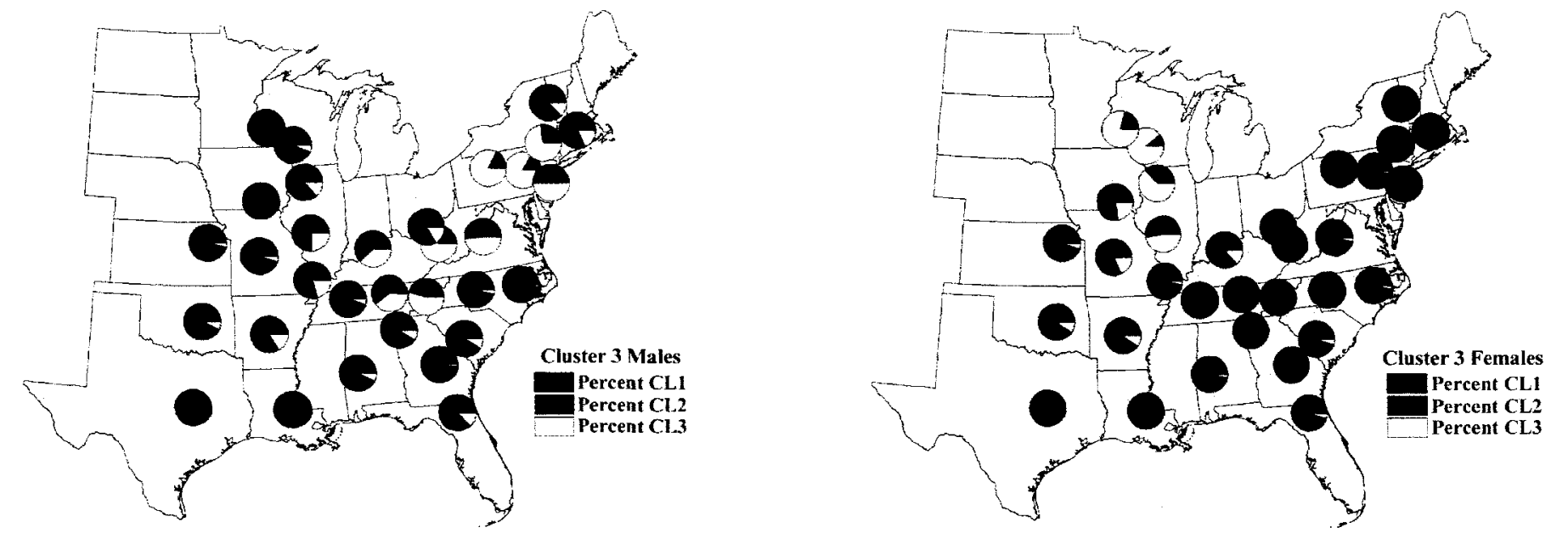

$\mathbf{B}$

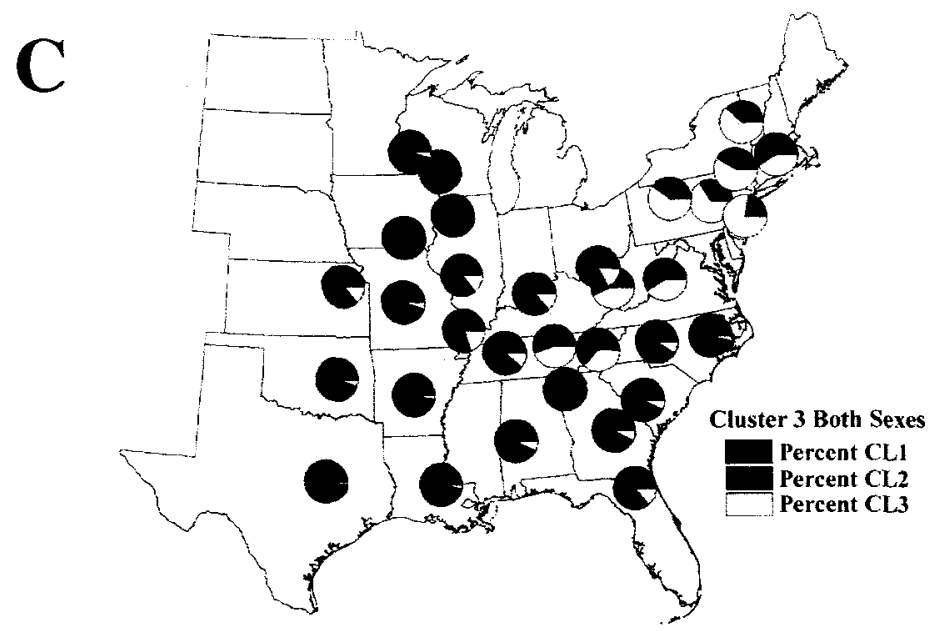

Fig. 30.-Maps of geographic clusters as percentages of regional populations for the three-cluster models. (A) Males. (B) Females. (C) Combined sexes. Each pie chart corresponds to one of the 32 regions shown in Figures 3 and 4. 
The three-cluster model for combined sexes produced three widespread clusters similar to those for males, but exhibited even greater overlap among clusters (Fig. 30). A map of clusters as a percentage of regional populations revealed two patterns of clinal variation, one strong and one weak, similar to those for males (Fig. 28C).

The four-cluster and five-cluster models for males, females, and combined sexes produced clusters with similar distributions to those of the two-cluster and three-cluster models. The same regions were moderately distinct: the northeastern and central-eastern regions, the central-western and southern regions, and the northwestern regions.

Additional clusters generated by these higher-order models did not add to the geographic distinctiveness of specimens from different regions in the two- and three-cluster models.

\section{Analysis of Morphometric Data}

Snout-Vent Length.-To determine whether adult snakes typically reached larger head or body sizes in some parts of the range than others, mature adults over $800 \mathrm{~mm}$ SVL were analyzed from the 12 selected study areas (Fig. 5). Larger SVL was detected in the southern regions for both males (Appendix XI) and females (Appendix XII). The effect of region was significant for males $(n=287 ; F=13.00 ; P<0.0001)$ and identified two groups: Group 1 (LAS-NCCP-FL-NCPD) had larger SVL than Group 2 (ILS-MONCMT-PAE-MNWI-NYN-KS-VAMT). The effect of region was significant for females $(n=222 ; F=24.43 ; P<0.0001)$ and identified two groups: Group 1 (NCCP-FL-LASNCPD) had larger SVL than Group 2 (NYN-ILS-MNWI-PAE-NCMT-KS-MO-VAMT). 
Head Length at Mandible._Larger HLM was detected in the southern regions for males (Appendix XI) and females (Appendix XII), using specimens greater than $800 \mathrm{~mm}$ SVL. The effect of region was significant for males $(n=266 ; F=15.90 ; P<0.0001)$ and identified two groups: Group 1 (LAS-NCCP-NCPD-FL) had larger HLM than Group 2 (NCMT-ILS-MO-KS-NYN-MNWI-VAMT-PAE). The effect of region was significant for females $(n=211 ; F=24.55 ; P<0.0001)$ and identified two groups: Group 1 (NCCPNCPD-LAS-FL) had larger HLM than Group 2 (NCMT-ILS-NYN-MNWI-KS-PAE-MOVAMT).

Part 1.-To test for the effect of sex, ANCOVA was performed on ten regressions using the entire data set (Table 2). Snout-vent length (SVL) explained significant variation in tail length (TL) for both males and females. Male TL increased with SVL at a much higher rate than female TL (Fig. 31). The SVL*sex interaction was significant, so the effect of sex alone could not be directly tested.

Snout-vent length explained significant variation in head length at the mandible (HLM) for both males and females (Table 2). The regressions of HLM on SVL did not differ greatly between males and females (Fig. 32A), but the SVL*sex interaction was significant. Similarly, SVL explained significant variation in head length at the supraoccipital bone (HLS) for males and females. The regressions of HLS on SVL between males and females were similar (Fig. 32B), but the SVL* sex interaction was significant.

Snout-vent length explained significant variation in quadrate length (QL) for both males and females (Table 2). The regressions of QL on SVL did not differ greatly between males and females (Fig. 33A), although the SVL*sex interaction was significant. SVL also explained significant variation in mandible length (ML) for both males and 
Table 2.-ANCOVA of ten morphometric relationships to test for the effects of sex using the entire data set. Character abbreviations are defined in Appendix II. The following data are reported for each relationship: sex ( $\mathrm{M}$ or $\mathrm{F}$ ), regression equation, percentage of variation explained $\left(r^{2}\right)$, sample size for each analysis $(n), F$-ratio $(F)$, and level of significance $(P)$.

\begin{tabular}{|c|c|c|c|c|c|c|}
\hline Relationship & Sex & Regression Equation & $r^{2}$ & $n$ & $F$ & $P$ \\
\hline \multirow[t]{2}{*}{ TL on SVL } & M & $\mathrm{TL}=2.35+0.0814 * \mathrm{SVL}$ & 0.958 & 975 & $22,041.9$ & 0.0001 \\
\hline & $\mathrm{F}$ & $\mathrm{TL}=4.19+0.0611 * \mathrm{SVL}$ & 0.937 & 859 & $12,813.3$ & 0.0001 \\
\hline SVL*sex & & & & 1,834 & 650.67 & 0.0001 \\
\hline \multirow[t]{2}{*}{ HLM on SVL } & M & $\mathrm{HLM}=10.22+0.0334 * \mathrm{SVL}$ & 0.970 & 933 & $30,074.2$ & 0.0001 \\
\hline & $\mathrm{F}$ & $\mathrm{HLM}=9.439+0.0342 * \mathrm{SVL}$ & 0.967 & 833 & $24,348.1$ & 0.0001 \\
\hline SVL*sex & & & & 1,766 & 7.91 & 0.005 \\
\hline \multirow[t]{2}{*}{ HLS on SVL } & M & $\mathrm{HLS}=9.48+0.0200 * \mathrm{SVL}$ & 0.962 & 920 & $23,422.5$ & 0.0001 \\
\hline & $\mathrm{F}$ & $\mathrm{HLS}=8.935+0.0204 * \mathrm{SVL}$ & 0.950 & 814 & $15,312.2$ & 0.0001 \\
\hline SVL* $\operatorname{sex}$ & & & & 1,734 & 4.38 & 0.037 \\
\hline \multirow[t]{2}{*}{ QL on SVL } & M & $\mathrm{QL}=2.13+0.0153 * \mathrm{SVL}$ & 0.964 & 925 & $24,801.9$ & 0.0001 \\
\hline & $\mathrm{F}$ & $\mathrm{QL}=1.67+0.0159^{*} \mathrm{SVL}$ & 0.964 & 822 & $21,701.4$ & 0.0001 \\
\hline SVL*sex & & & & 1,747 & 14.47 & 0.0001 \\
\hline \multirow[t]{2}{*}{ ML on SVL } & M & $\mathrm{ML}=8.96+0.0322 * \mathrm{SVL}$ & 0.973 & 873 & $31,774.2$ & 0.0001 \\
\hline & $\mathrm{F}$ & $\mathrm{ML}=8.20+0.0331 * \mathrm{SVL}$ & 0.968 & 779 & $23,187.4$ & 0.0001 \\
\hline$S V L * \operatorname{sex}$ & & & & 1,652 & 8.22 & 0.0042 \\
\hline \multirow[t]{2}{*}{ HLS on HLM } & M & HLS $=3.63+0.591 *$ HLM & 0.973 & 926 & $32,746.8$ & 0.0001 \\
\hline & $\mathrm{F}$ & $\mathrm{HLS}=3.58+0.589 * \mathrm{HLM}$ & 0.965 & 811 & $22,043.8$ & 0.0001 \\
\hline$H_{L} M^{*} \operatorname{sex}$ & & & & 1,737 & 0.20 & 0.66 \\
\hline Sex & & & & 1,737 & 8.65 & 0.0033 \\
\hline \multirow[t]{2}{*}{ HW on HLM } & M & $\mathrm{HW}=-2.61+0.810 * \mathrm{HLM}$ & 0.955 & 539 & $11,366.4$ & 0.0001 \\
\hline & $\mathrm{F}$ & $\mathrm{HW}=-2.41+0.800 * \mathrm{HLM}$ & 0.946 & 451 & $7,881.5$ & 0.0001 \\
\hline$H_{L}{ }^{*} \operatorname{sex}$ & & & & 990 & 0.81 & 0.37 \\
\hline $\operatorname{Sex}$ & & & & 990 & 3.22 & 0.073 \\
\hline \multirow[t]{2}{*}{ QL on HLM } & $\mathrm{M}$ & $\mathrm{QL}=-2.37+0.454 * \mathrm{HLM}$ & 0.976 & 917 & $37,874.1$ & 0.0001 \\
\hline & $\mathrm{F}$ & $\mathrm{QL}=-2.58+0.461 * \mathrm{HLM}$ & 0.974 & 807 & $29,748.7$ & 0.0001 \\
\hline$H_{L}{ }^{*} \operatorname{sex}$ & & & & 1,724 & 3.29 & 0.07 \\
\hline Sex & & & & 1,724 & 0.91 & 0.34 \\
\hline \multirow[t]{2}{*}{ ML on HLM } & M & $\mathrm{ML}=-.0745+0.961 * \mathrm{HLM}$ & 0.989 & 883 & $82,421.6$ & 0.0001 \\
\hline & $\mathrm{F}$ & $\mathrm{ML}=-0.656+0.958 * \mathrm{HLM}$ & 0.988 & 778 & $63,737.8$ & 0.0001 \\
\hline $\mathrm{HLM}^{*} \mathrm{sex}$ & & & & 1,661 & 0.32 & 0.57 \\
\hline Sex & & & & 1,661 & 0.13 & 0.72 \\
\hline \multirow[t]{2}{*}{$\mathrm{QL}$ on $\mathrm{ML}$} & M & $\mathrm{QL}=-2.01+0.472 * \mathrm{ML}$ & 0.981 & 866 & 43.554 .9 & 0.0001 \\
\hline & $\mathrm{F}$ & $\mathrm{QL}=-2.16+0.478 * \mathrm{ML}$ & 0.977 & 767 & $32,402.8$ & 0.0001 \\
\hline$M L * \operatorname{sex}$ & & & & 1,633 & 2.64 & 0.10 \\
\hline Sex & & & & 1,633 & 1.63 & 0.20 \\
\hline
\end{tabular}




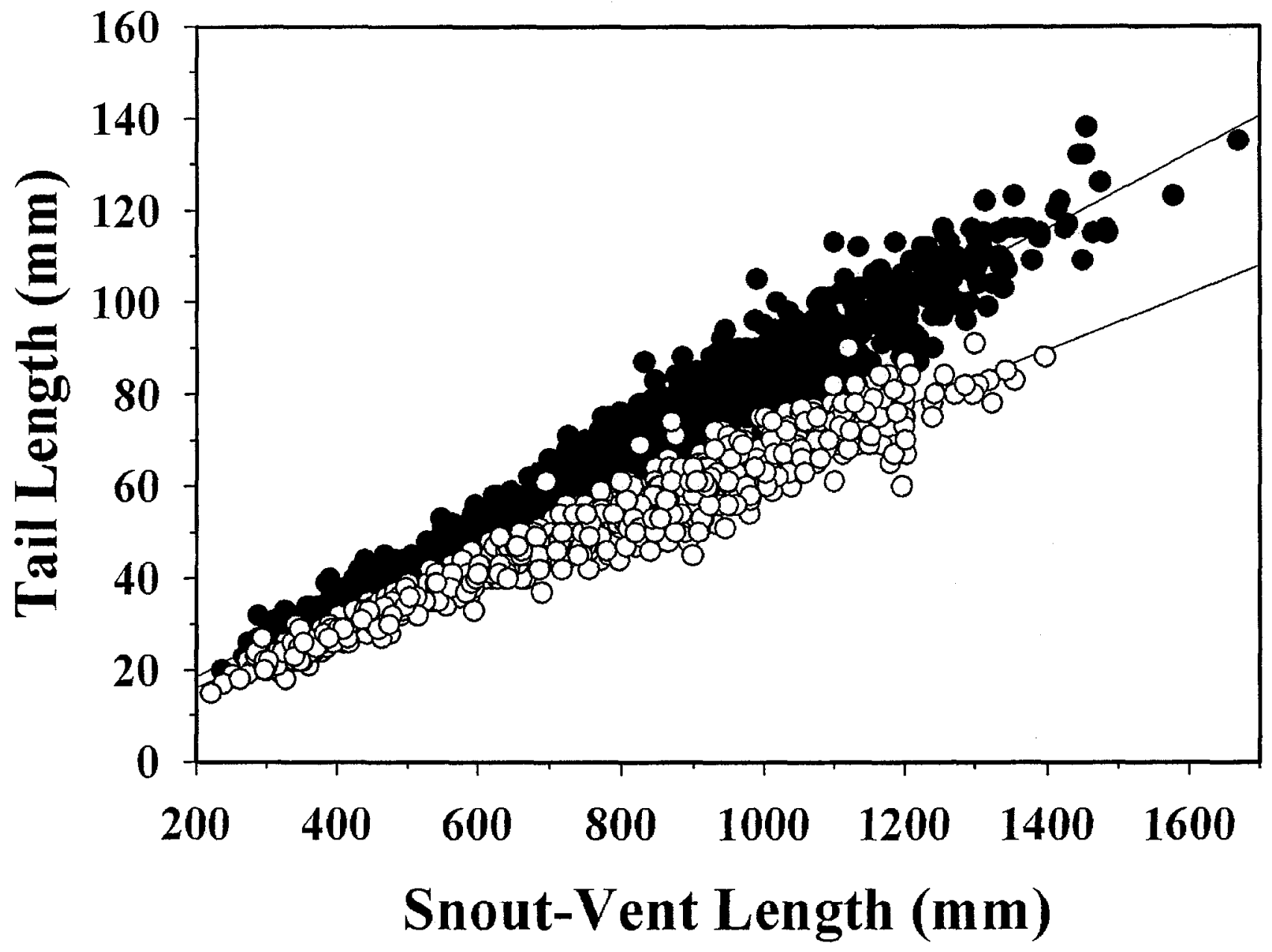

Fig. 31.-Regression of tail length on snout-vent length. Closed circles = males; open circles = females. Regression equations and statistics are provided in Table 2. 

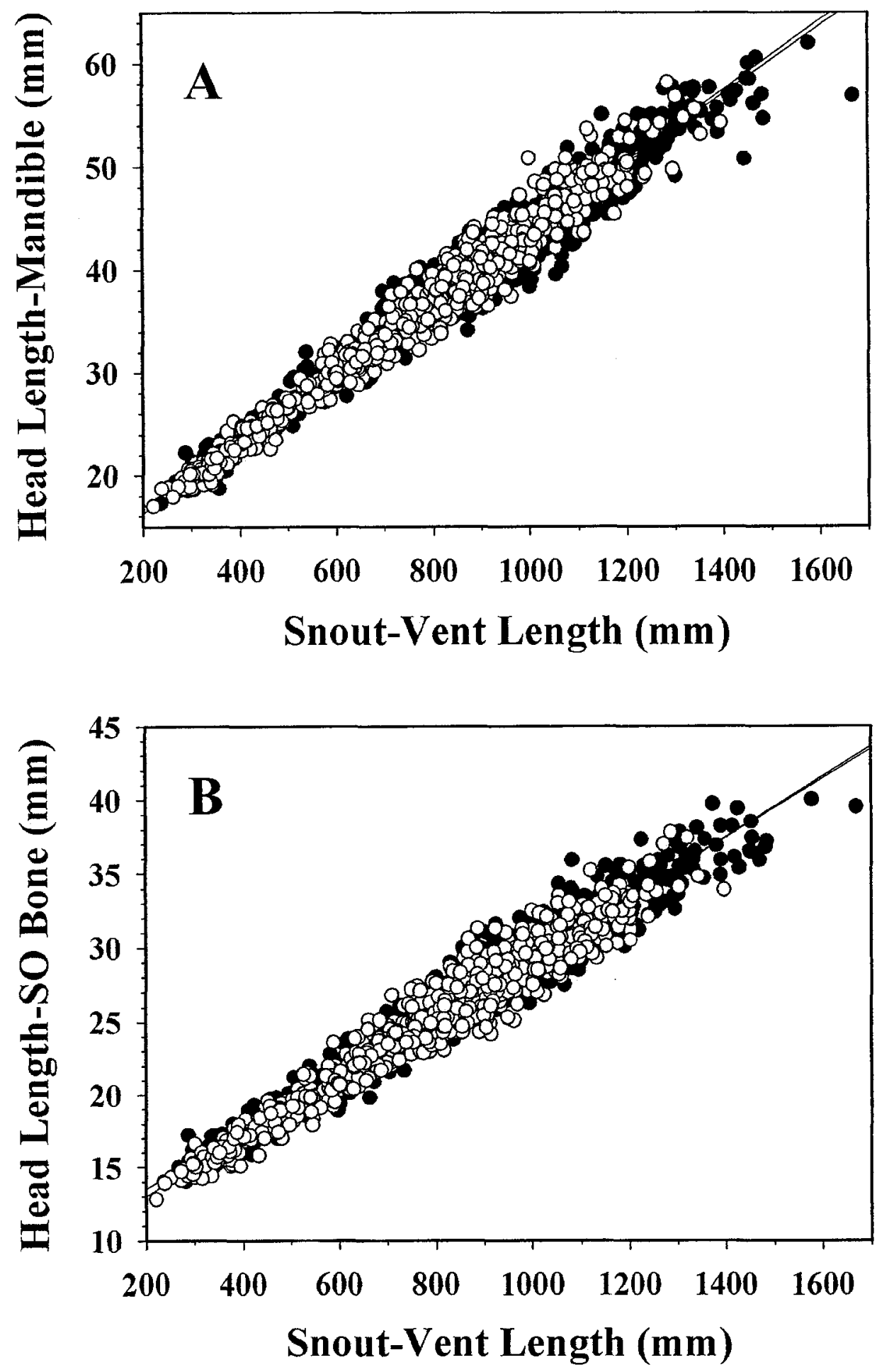

Fig. 32.- Regression of head lengths on snout-vent length. (A) Head length at the mandible on snout-vent length. (B) Head length at the SO (supraoccipital) bone on snout-vent length. Closed circles $=$ males; open circles $=$ females. Regression equations and statistics are provided in Table 2. 

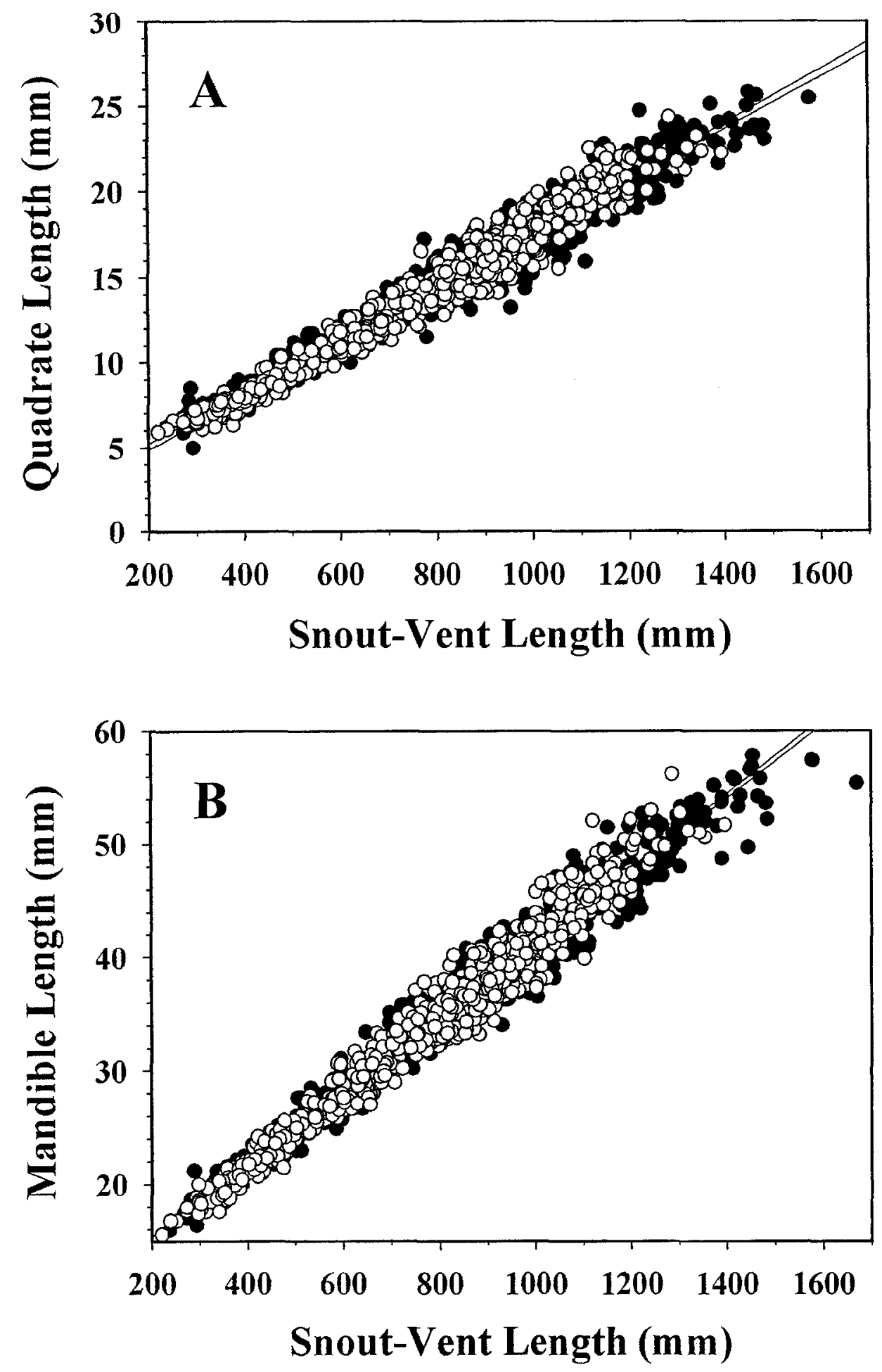

Fig. 33.- Regression of quadrate and mandible lengths on snout-vent length.

(A) Quadrate length on snout-vent length. (B) Mandible length on snout-vent length. Closed circles $=$ males; open circles $=$ females. Regression equations and statistics are provided in Table 2 . 

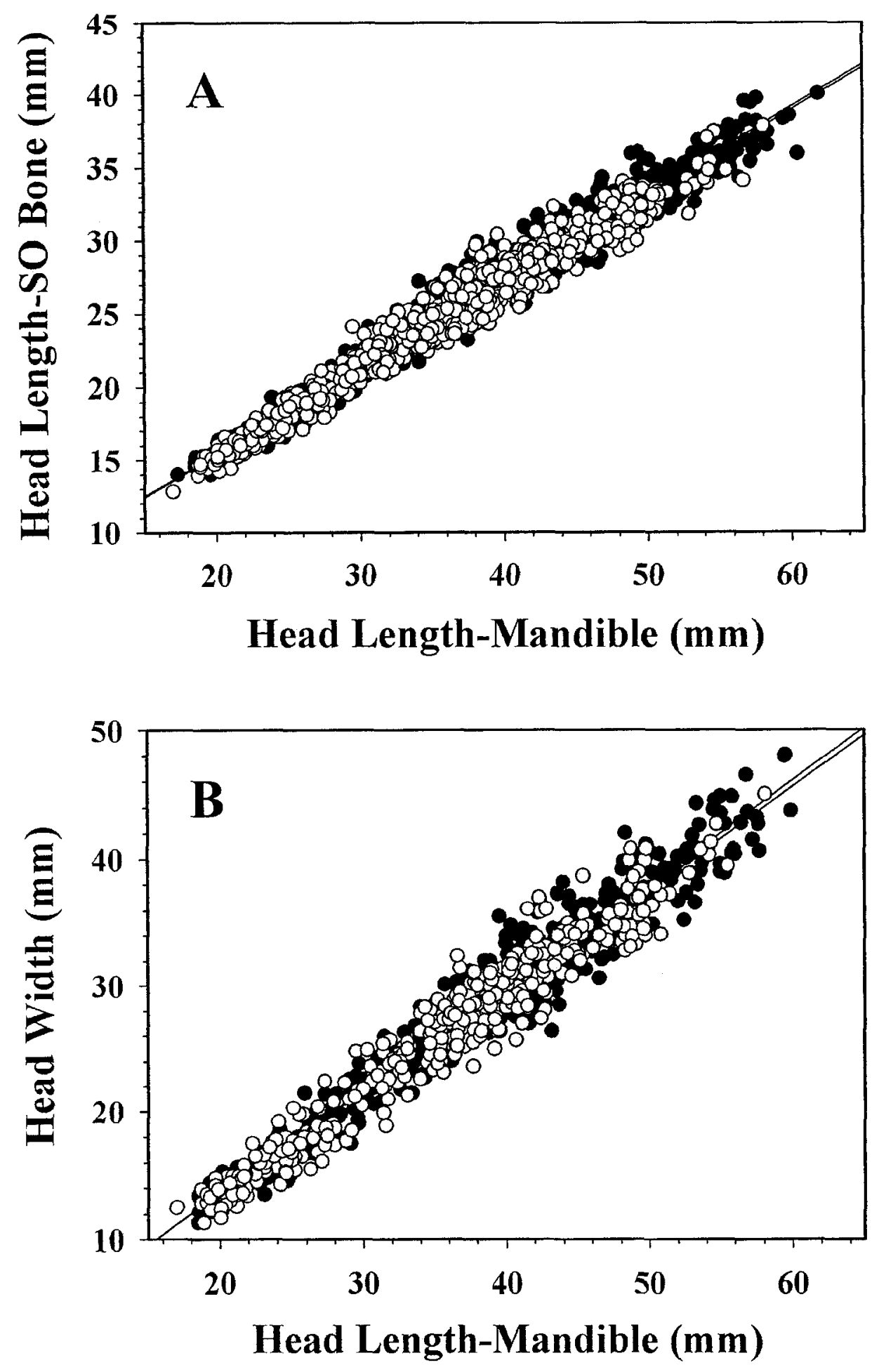

Fig. 34.- Regression of head length at the supraoccipital bone and head width on head length. (A) Head length at the SO (supraoccipital) bone on head length at the mandible. (B) Head width on head length at the mandible. Closed circles = males; open circles = females. Regression equations and statistics are provided in Table 2. 

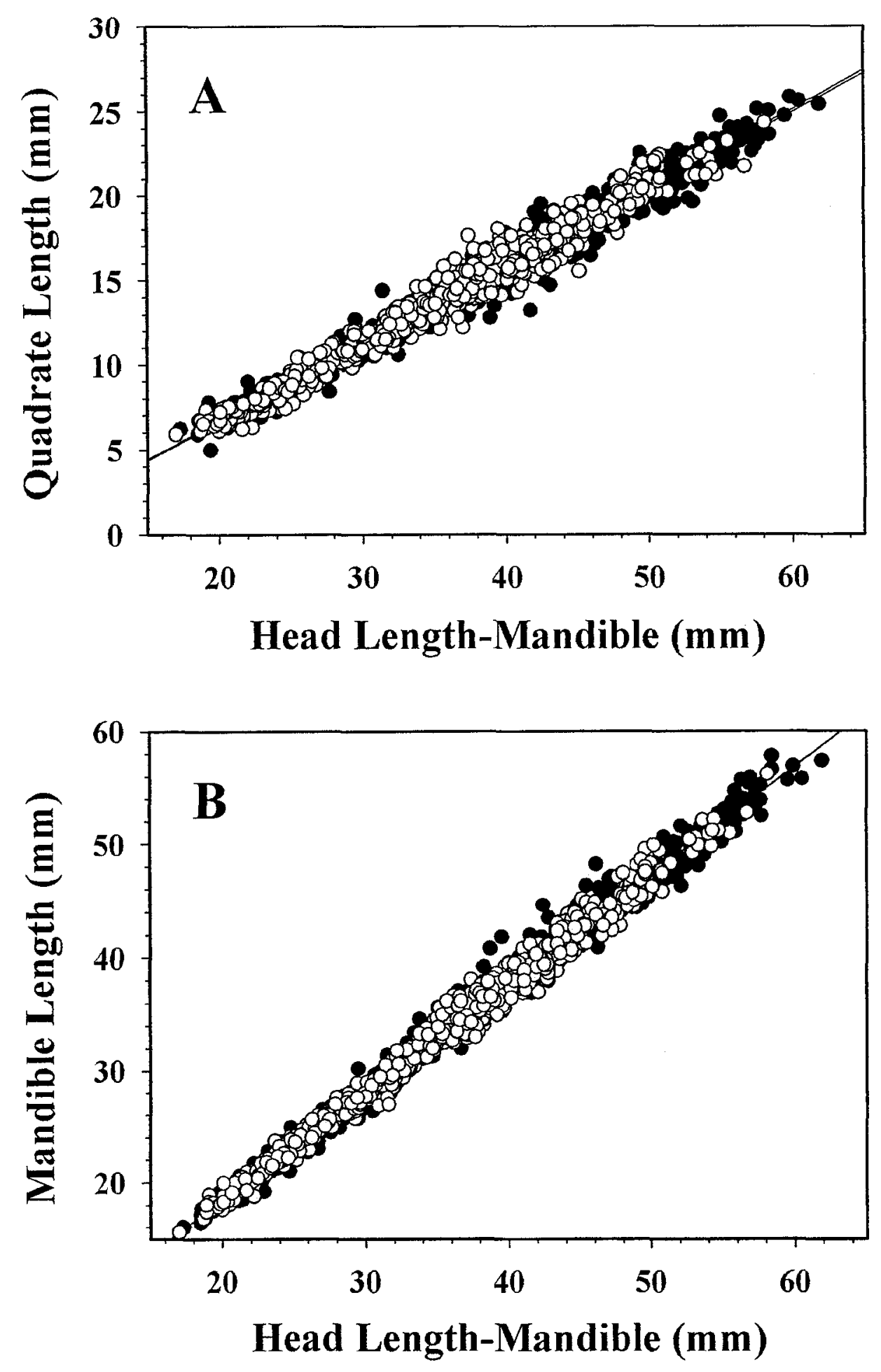

Fig. 35.-Regression of quadrate and mandible lengths on head length at the mandible. (A) Quadrate length on head length at the mandible. (B) Mandible length on head length at the mandible. Closed circles = males; open circles $=$ females. Regression equations and statistics are provided in Table 2. 


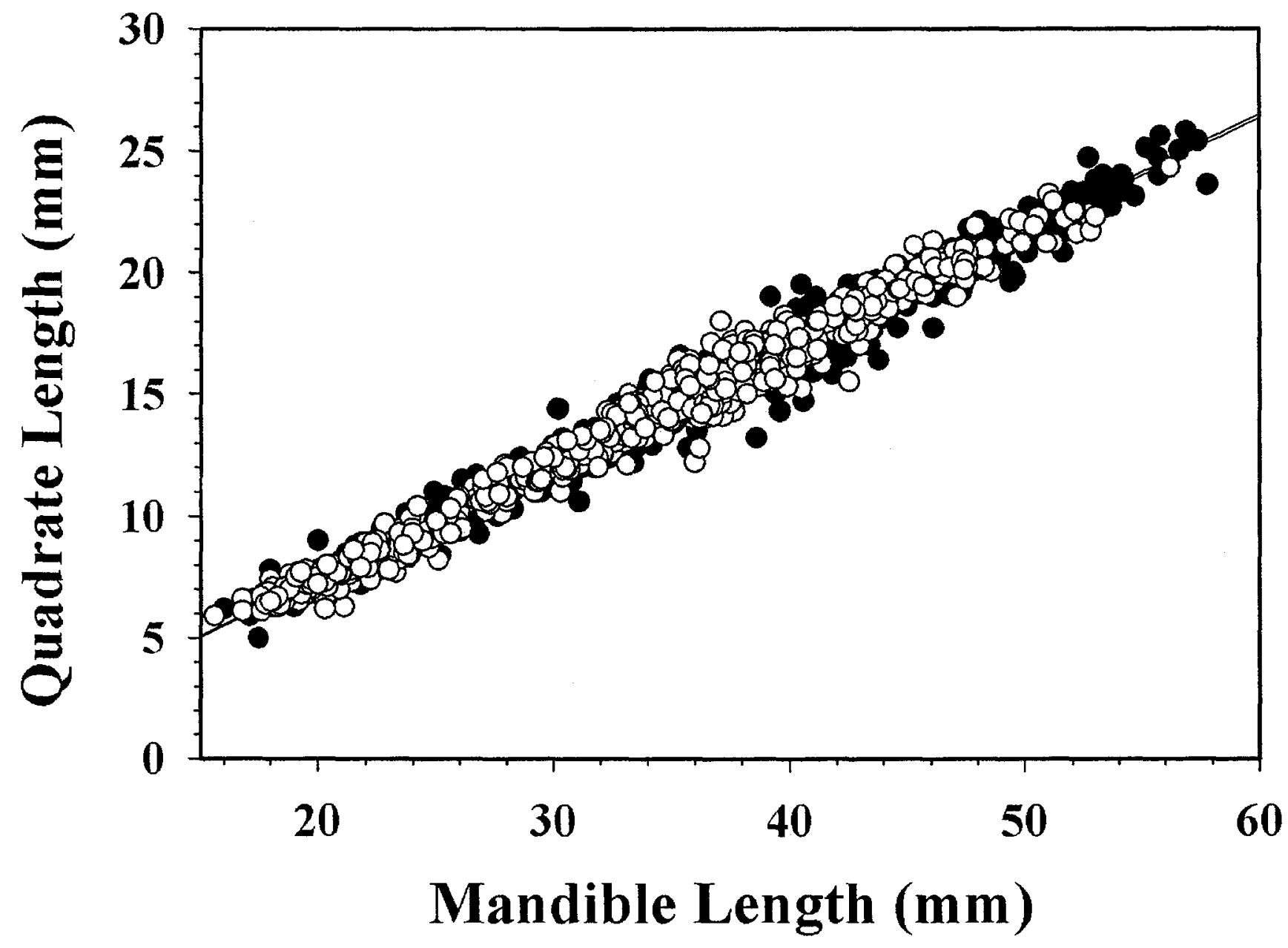

Fig. 36.--Regression of quadrate length on mandible length. Closed circles = males; open circles $=$ females. Regression equations and statistics are provided in Table 2 . 
females. The regressions of ML on SVL differed little between males and females (Fig. 33B), but the SVL* sex interaction was significant.

Head length at the mandible (HLM) explained significant variation in head length at the supraoccipital bone (HLS) for males and females (Table 2). The regressions of HLS on HLM were similar between males and females (Fig. 34A). The HLM*sex interaction was not significant, but the effect of sex was significant. HLM also explained significant variation in head width (HW) for males and females. The regressions of HW on HLM were similar between males and females (Fig. 34B). Neither the HLM*sex interaction nor the effect of sex was significant.

HLM explained significant variation in QL for males and females (Table 2). The regressions of QL on HLM were similar between males and females (Fig. 35A). Neither the HLM* sex interaction nor the effect of sex was significant. HLM also explained significant variation in ML for males and females. The regressions of ML on HLM did not differ between males and females (Fig. 35B). Neither the HLM*sex interaction nor the effect of sex was significant.

Mandible length (ML) explained significant variation in QL for males and females (Table 2). The regressions of QL on ML were similar for males and females (Fig. 36). Neither the $M L^{*}$ sex interaction nor the effect of sex was significant.

Part 2.-To test for the effect of sex in smaller but more geographically homogeneous groups, ANCOVA was performed on the ten regressions using the combined data set from the 12 selected study areas (Fig. 5). For TL on SVL, male TL increased with SVL at a much higher rate than female TL, and the SVL*sex interaction was significant (Table 3). The SVL*sex interactions were also significant for HLM on 
Table 3.-ANCOVA of ten morphometric relationships to test for the effects of sex using data from the 12 selected study areas (Fig. 5). Character abbreviations are defined in Appendix II. The following data are reported for each relationship: effect, sample size for each analysis $(n), F$-ratio $(F)$, and level of significance $(P)$.

\begin{tabular}{|c|c|c|c|c|}
\hline Relationship & Effect & $n$ & $F$ & $P$ \\
\hline TL on SVL & SVL*sex & 834 & 285.18 & 0.0001 \\
\hline HLM on SVL & $\mathrm{SVL}^{*} \mathrm{sex}$ & 811 & 9.02 & 0.0028 \\
\hline HLS on SVL & $S V L *$ sex & 795 & 9.77 & 0.0018 \\
\hline QL on SVL & $\mathrm{SVL}^{*} \operatorname{sex}$ & 794 & 11.99 & 0.0006 \\
\hline ML on SVL & SVL*sex & 761 & 16.78 & 0.0001 \\
\hline \multirow[t]{2}{*}{ HLS on HLM } & HLM $^{*} \operatorname{sex}$ & 796 & 0.16 & 0.69 \\
\hline & Sex & 796 & 2.58 & 0.11 \\
\hline HW on HLM & $\mathrm{HLM}^{*} \operatorname{sex}$ & 462 & 4.15 & 0.042 \\
\hline \multirow[t]{2}{*}{ QL on HLM } & HLM $^{*} \operatorname{sex}$ & 788 & 2.04 & 0.15 \\
\hline & Sex & 788 & 0.02 & 0.89 \\
\hline \multirow[t]{2}{*}{ ML on HLM } & HLM*sex & 760 & 1.28 & 0.26 \\
\hline & Sex & 760 & 0.51 & 0.48 \\
\hline \multirow[t]{2}{*}{$\mathrm{QL}$ on $\mathrm{ML}$} & $M L^{*} \operatorname{sex}$ & 746 & 0.19 & 0.66 \\
\hline & Sex & 746 & 0.51 & 0.47 \\
\hline
\end{tabular}


SVL, HLS on SVL, QL on SVL, and ML on SVL, but males and females did not differ greatly in these regressions.

For HLS on HLM, neither the HLM*sex interaction nor the effect of sex was significant (Table 3). For HW on HLM, HW increased with HLM at a higher rate in males than in females and the HLM*sex interaction was significant. For QL on HLM, neither the HLM*sex interaction nor the effect of sex was significant. Similarly, for ML on HLM, neither the HLM*sex interaction nor the effect of sex was significant. For QL on $\mathrm{ML}$, neither the $\mathrm{ML}^{*}$ sex interaction nor the effect of sex was significant.

Part 3.- To test for the effect of region, ANCOVA was performed for males, females, and combined sexes using the combined data set from the 12 selected study areas (Fig. 5). Among males, for TL on SVL, the SVL*region interaction was significant, so the effect of region alone could not be directly tested (Table 4). For HLM on SVL, the SVL*region interaction was not significant, but the effect of region was significant and identified three groups: Group 1 (FL-NCCP), Group 2 (NCMT-LAS-VAMT-NYN), and Group 3 (PAE-NCPD-ILS-MNWI-MO-KS). For HLS on SVL, the SVL*region interaction was not significant, but the effect of region was significant and identified four groups: Group 1 (FL-NCCP), Group 2 (NCMT), Group 3 (VAMT-LAS-NCPD-PAENYN-MNWI-ILS), and Group 4 (MO-KS). For QL on SVL, the SVL*region interaction was not significant, but the effect of region was significant and identified four groups: Group 1 (FL-NCCP), Group 2 (NCMT-VAMT-LAS-NYN), Group 3 (PAE-NCPDMNWI-ILS), and Group 4 (MO-KS). For ML on SVL, the SVL*region interaction was not significant, but the effect of region was significant and identified five groups: Group 1 (FL-NCCP), Group 2 (NCMT-LAS), Group 3 (VAMT-NYN-NCPD), Group 4 (PAE- 
Table 4.-ANCOVA of ten morphometric relationships to test for the effects of region using data from the 12 selected study areas (Fig. 5). Character abbreviations are defined in Appendix II. The following data are reported for males, females, and combined sexes: relationship, effect, sample size for each analysis $(n), F$-ratio $(F)$, and level of significance $(P)$.

\begin{tabular}{|c|c|c|c|c|c|}
\hline Sex & Relationship & Effect & $n$ & $F$ & $P$ \\
\hline \multirow[t]{11}{*}{ Males } & TL on SVL & SVL*region & 452 & 2.69 & 0.0024 \\
\hline & \multirow[t]{2}{*}{ HLM on SVL } & SVL*region & 438 & 0.97 & 0.47 \\
\hline & & Region & 438 & 8.80 & 0.0001 \\
\hline & \multirow[t]{2}{*}{ HLS on SVL } & SVL*region & 428 & 0.90 & 0.54 \\
\hline & & Region & 428 & 3.63 & 0.0001 \\
\hline & \multirow[t]{2}{*}{ QL on SVL } & SVL*region & 423 & 1.67 & 0.078 \\
\hline & & Region & 423 & 6.09 & 0.0001 \\
\hline & \multirow[t]{2}{*}{ ML on SVL } & SVL ${ }^{*}$ region & 406 & 0.66 & 0.78 \\
\hline & & Region & 406 & 7.75 & 0.0001 \\
\hline & \multirow[t]{2}{*}{ HW on HLM } & HLM* $^{*}$ region & 249 & 0.71 & 0.73 \\
\hline & & Region & 249 & 1.78 & 0.058 \\
\hline \multirow[t]{10}{*}{ Females } & \multirow[t]{2}{*}{ TL on SVL } & SVL*region & 382 & 0.92 & 0.53 \\
\hline & & Region & 382 & 1.78 & 0.057 \\
\hline & \multirow[t]{2}{*}{ HLM on SVL } & SVL*region & 373 & 0.94 & 0.50 \\
\hline & & Region & 373 & 9.00 & 0.0001 \\
\hline & \multirow[t]{2}{*}{ HLS on SVL } & SVL*region & 367 & 0.46 & 0.93 \\
\hline & & Region & 367 & 5.80 & 0.0001 \\
\hline & QL on SVL & SVL*region & 371 & 3.33 & 0.0002 \\
\hline & ML on SVL & SVL*region & 355 & 1.93 & 0.035 \\
\hline & \multirow[t]{2}{*}{ HW on HLM } & HLM*region & 213 & 0.73 & 0.71 \\
\hline & & Region & 213 & 2.71 & 0.0028 \\
\hline \multirow[t]{8}{*}{ Combined Sexes } & \multirow[t]{2}{*}{ HLS on HLM } & HLM $^{*}$ region & 796 & 0.53 & 0.88 \\
\hline & & Region & 796 & 4.32 & 0.0001 \\
\hline & \multirow[t]{2}{*}{ QL on HLM } & HLM*region $^{*}$ & 788 & 1.46 & 0.14 \\
\hline & & Region & 788 & 1.96 & 0.030 \\
\hline & \multirow[t]{2}{*}{ ML on HLM } & $\mathrm{HLM}^{*}$ region & 760 & 0.95 & 0.49 \\
\hline & & Region & 760 & 7.40 & 0.0001 \\
\hline & \multirow[t]{2}{*}{ QL on $M L$} & ML*region & 746 & 1.38 & 0.18 \\
\hline & & Region & 746 & 3.71 & 0.0001 \\
\hline
\end{tabular}


MNWI-ILS), and Group 5 (MO-KS). For HW on HLM, the HLM*region interaction and the effect of region were not significant.

Among females, for TL on SVL, the SVL*region interaction and the effect of region were not significant (Table 4). For HLM on SVL, the SVL*region interaction was not significant, but the effect of region was significant and identified six groups: Group 1 (NCCP), Group 2 (NCPD), Group 3 (FL-PAE-MNWI), Group 4 (VAMT-NCMT-NYN), Group 5 (LAS-MO-KS), and Group 6 (ILS). For HLS on SVL, the SVL*region interaction was not significant, but the effect of region was significant and identified five groups: Group 1 (NCCP), Group 2 (NCPD), Group 3 (PAE), Group 4 (VAMT-FLMNWI-NCMT-NYN), and Group 5 (MO-LAS-KS-ILS). For QL on SVL, the SVL*region interaction was significant, and for ML on SVL, the SVL*region interaction was significant. For HW on HLM, the HLM*region interaction was not significant, but the effect of region was significant and identified three groups: Group 1 (NCPD-NCCP), Group 2 (NYN-LAS-PAE-MNWI-VAMT), and Group 3 (ILS-FL-KS-NCMT-MO).

Among combined sexes, for HLS on HLM, the HLM*region interaction was not significant, but the effect of region was significant (Table 4) and identified five groups: Group 1 (NCCP), Group 2 (FL), Group 3 (NCPD-VAMT-NCMT-PAE), Group 4 (LASNYN-MNWI), and Group 5 (MO-ILS-KS). For QL on HLM, the HLM*region interaction was not significant, but the effect of region was significant and identified five groups: Group 1 (NCCP), Group 2 (FL), Group 3 (NCPD-NCMT-VAMT-PAE-MNWILAS), Group 4 (NYN), and Group 5 (MO-ILS-KS). For ML on HLM, the HLM*region interaction was not significant, but the effect of region was significant and identified seven groups: Group 1 (NCCP), Group 2 (FL), Group 3 (NCPD), Group 4 (VAMT- 
NCMT), Group 5 (LAS-PAE), Group 6 (MNWI-NYN), and Group 7 (KS-MO-ILS). For $\mathrm{QL}$ on $\mathrm{ML}$, the $\mathrm{ML} *$ region interaction was not significant, but the effect of region was significant and identified six groups: Group 1 (NCCP), Group 2 (FL-NCPD), Group 3 (NCMT-VAMT), Group 4 (PAE-MNWI-LAS), Group 5 (NYN), and Group 6 (MO-KSILS).

In summary, sexual dimorphism was large for the regression of tail length on snout-vent length, and small or nonexistent for the regressions of the various head dimensions on snout-vent length and on head length at the mandible. Morphometric data exibited a strong north-south pattern of geographic variation; head and body measurements were generally larger in the south. The regression of tail length on snoutvent length could not be tested for regional effects because of significant interaction effects. Different ANCOVAs among the 12 study areas showed that head dimensions generally were largest in FL, NCCP, and NCPD and smallest in ILS, FL, KS, LAS, MO, and NCMT after being adjusted for SVL or HLM.

Factor Analysis.--Factor analysis was performed on the entire morphometric data set to detect common factors that characterize overall size and shape. Factor 1 explained $97 \%$ of the variation in the model (Appendix 13). All six morphometric characters loaded strongly and positively on Factor 1, which corresponded to a measure of overall size. Although the other three factors explained only $3 \%$ of the variation in the model, their loadings may be informative. Factor 2 exhibited a relatively high positive loading of TL against low negative loadings for SVL, HLM, HLS, QL, and ML. Factor 3 was characterized by a relatively high positive loading for HLS against low negative loadings 
of SVL, TL, HLM, QL, and ML. Factor 4 exhibited a relatively high negative loading for SVL against low positive loadings for TL, HLM, HLS, QL, and ML.

A two-way ANOVA was used to test Factor 1 for the effects of region and sex. The region* ${ }^{*}$ sex interaction was not significant $(n=1504 ; F=4.37 ; P<0.0001)$, but the effect of sex was significant $(F=31.21 ; P<0.0001)$. Males were significantly larger $(n=$ 797 ; mean $=0.1405)$ than females $(n=707 ;$ mean $=-0.1584)$. The effect of region was significant $(F=5.52 ; P<0.0001)$, but the REGWQ multiple range test was ambiguous and identified significant differences only between two small groups: NCCP-FL-LAMI and CTMA-KSNE. These results identify a difference in overall size between specimens in the extreme south and specimens in the northeastern and central-western regions. 


\section{DISCUSSION}

\section{Analysis of Meristic Data}

This study revealed that morphological variation within Crotalus horridus was more complex than previously demonstrated (Brown and Ernst 1986; Gloyd 1935, 1940; Martin, 1992a; Pisani et al., 1973). Variation due to sexual dimorphism was examined first to separate it from variation that was strictly geographic. Significant sexual dimorphism was observed in 13 meristic characters, but that high number of significant characters reflects the large sample size. The $t$-tests using all data had substantial power and were more likely to be significant because the large data set increased the degrees of freedom and decreased the minimum significant difference detectable between the sexes. The actual magnitude of the differences between sexes was large only for subcaudal scale number (CS) and band length (BL), moderate for ventral scale number (VS) and band spacing (BS), and small for the rest of the dimorphic characters. Males had larger CS, whereas females had larger VS, BL, and BS. The $t$-tests on the 12 selected study areas also revealed sexual dimorphism; these independent tests provided verification and were based on moderate sample sizes. Two characters, CS and VS, exhibited significant sexual dimorphism in each study area and should be considered strong dimorphic characters. Conversely, BL and BS did not display sexual dimorphism in every population and should be considered weak to moderate sexually dimorphic characters. Similar differences between males and females in CS and VS are well documented in other species of snakes (Ernst, 1989, 1992; Klauber, 1972; Shine, 1993). 
Substantial geographic variation within Crotalus horridus was observed clearly in all individual meristic characters. Scutellation showed a general north-south pattern of geographic variation for most characters. Dorsal scale rows (ADS, MDS, PDS), ventral and subcaudal scales (VS, male CS), and infralabial scales (LIL, RIL) averaged higher in the southern regions. This general latitudinal pattern of scutellation seemed to be modified by elevation. Geographic variation in scutellation probably is influenced by selection in response to differing suites of environmental variables (Cracraft, 1989; Endler, 1977; Gould and Johnston, 1972; Thorpe et al., 1995). Aspects of the physical environment that vary by latitude and elevation include surface temperatures, and thus the length of the active season, humidity, and precipitation.

Climatically, the range of Crotalus horridus probably imposes various selective pressures on the species. The Köppen classification system divides the mid-latitude climates, which correspond to the distribution of $C$. horridus, into two climatic regions (Ahrens, 1994). The humid subtropical region (Cfa) is located at latitude $25-40^{\circ} \mathrm{N}$ and experiences mild winters. The humid continental region (Dfab) is located at latitude 40$50^{\circ} \mathrm{N}$ and experiences severe winters. The western boundaries of each of those regions roughly coincide with the western limits of $C$. horridus. The annual temperature regimes of each region are different (Ahrens, 1994). Mean temperature of the coldest month is between $-3 \mathrm{C}$ and $18 \mathrm{C}$ for $\mathrm{Cfa}$ and $-3 \mathrm{C}$ or below for $\mathrm{Dfab}$. Mean temperature of the warmest month is above $22 \mathrm{C}$ for $\mathrm{Cfa}$ and $\mathrm{Dfa}$, but the mean temperatures of all months are below $22 \mathrm{C}$ for $\mathrm{Dfb}$. Mean annual precipitation is roughly divided into two regions along a diagonal line from eastern Texas to Maine (Ahrens, 1994; Barry and Chorley, 1998); the southwestern, central, and eastern Cfa and coastal Dfab receive $100-200 \mathrm{~cm}$ of 
annual precipitation, compared to $50-100 \mathrm{~mm}$ in the northwestern Cfa and interior Dfab. Higher elevations are characterized by a combination of lower mean annual temperatures and higher amounts of annual precipitation than lower elevations at the same latitude (Barry and Chorley, 1998).

The general north-south pattern of scutellation appears to follow mean annual temperature south of the division between Cfa and Dfab. However, at the higher elevations of the southern Appalachian Mountains low values of ADS, MDS, PDS, and VS characteristic of the north occur much farther south than in adjacent regions east and west of the mountains. Variation in scutellation does not appear to reflect the pattern of precipitation across the two climatic regions. Two characters of the scutellation showed very different patterns of variation from the other characters. Female subcaudal scale number (CS) had unusually high values in the southwestern region and low values elsewhere. CS may be subject to high levels of sexual selection, which could easily obscure other patterns of variation, but the total range of variation in female CS was small and the geographic pattern may even be random. Both left and right supralabial counts (LSL, RSL) had low values concentrated in the northwestern region and high values in the remaining distribution. High values of LSL and RSL in the northeastern region are difficult to interpret. Likewise, high values of male and female VS and male CS occur in snakes from northern New York. These characters may reflect genetic drift in relatively small and isolated peripheral metapopulations.

Higher scale counts in the southern regions could also be the result of selection for larger body sizes, which in turn may reflect longer annual growing periods in southern regions. The number of dorsal scale rows may be directly related to girth and 
may be associated with prey size (Pough and Groves, 1983; but see Shine, 2002).

Smaller mammals, such as Peromyscus and Tamias, may be consumed more frequently by Crotalus horridus at higher latitudes and elevations, whereas populations at lower latitudes and elevations may feed more frequently on larger prey, such as Sciurus. Warmer ambient temperatures may promote more rapid rates of digestion, resulting in higher growth rates. Viperids possess long mandibles compared to other snakes (Cundall, 2002), facilitating consumption of larger prey. Although variation in the number of labial scales may be expected to reflect gape, no consistent pattern was found in the usual morphometric correlates of gape, such as quadrate and mandible length.

Many ophidians display latitudinal patterns of geographic variation in scutellation. Christman (1980) examined 15 species of snakes in Florida and found a latitudinal pattern of geographic variation in two characters, ventral and subcaudal scales, both of which increased in number from north to south in most species. The significant latitudinal variation within populations limited to peninsular Florida suggests that geographic variation in scutellation may be even greater for the larger and more heterogeneous continental populations. Despite some regional divergences, ventral, subcaudal, and dorsal scale numbers generally increase from north to south for continental populations of Elaphe guttata (Mitchell, 1977; Thomas, 1974), Lampropeltis getula (Blaney, 1971), L. triangulum (Williams, 1978), and Thamnophis sirtalis (Fitch, 1941); only subcaudals show this pattern in T. proximus (Rossman, 1962) and T. sauritus (Rossman, 1963). Divergences from the general pattern in Lampropeltis triangulum may reflect the effects of elevation and habitat. 
Geographic variation in the scutellation of other species of snakes is associated with levels of annual precipitation. Gloyd (1940) found geographic variation in the number of dorsal scale rows in several species of rattlesnakes. Within the Crotalus atrox group, C. adamanteus shows higher numbers of dorsal scales than C. atrox and inhabits moister regions with higher levels of precipitation. However, mainland C. exsul also exhibits relatively high numbers of dorsal scales, although it inhabits dry, rocky habitats with much lower levels of precipitation than does $C$. adamanteus. Sistrurus miliarius has higher numbers of dorsal scale rows in eastern versus western populations, but only populations in the extreme western part of its range experience lower precipitation. Sistrurus catenatus shows higher numbers of dorsal scale rows in the northeastern versus southwestern parts of its range; the southwestern populations experience relatively lower annual precipitation.

Conversely, Castellano et al. (1994) examined microgeographic variation in Trimeresurus stejnegeri on Taiwan and reported that higher numbers of body scales are associated with regions of lower precipitation. Burbrink (2001) found significant patterns of geographic variation in scutellation within the Elaphe obsoleta complex. The number of dorsal scales was lower in the central clade, higher in the eastern clade, and highest in the western and xeric clades, which receive less precipitation. The number of ventral and subcaudal scales decreased from the eastern to the western clade, but increased greatly from the western to the xeric clade. The number of body scales also increases in regions of lower precipitation in the lizards Anolis oculatus (Malhotra and Thorpe, 1991), Gallotia galloti (Thorpe and Baez, 1987), and Sceloporus variabilis (Sites and Dixon, 1982). Moreover, higher numbers of dorsal scales in G. galloti are correlated with higher 
temperatures, lower latitudes, and lower elevations (Thorpe and Baez, 1987). Thus, characters of the scutellation partially coincide with patterns of annual precipitation, but geographic patterns of scutellation can be highly variable among species.

Explaining the adaptive significance, if any, of such broad patterns of geographic variation in scutellation is difficult. First, it is unclear which environmental factors are directly responsible for geographic variation because climatic factors may only be correlated with the actual causative factors, and the latter may not even be included in the study. Second, environmental factors often are highly correlated with each other, precluding statistical testing of single factors. For example, the climate throughout most of the range of Crotalus horridus displays either high annual temperatures and high precipitation or low temperatures and low precipitation. The combinations of higher temperatures and lower precipitation (or vice versa) are underrepresented, making it difficult to separate the effects of each single factor. Phenotypic plasticity can also modify traits that generally are determined genetically. Differences in scutellation can be modified by developmental conditions. Previous studies have shown that temperature affects the development of meristic characters in Nerodia fasciata (Osgood, 1978) and Thamnophis elegans (Fox et al., 1961; Peterson et al., 1993). Common garden experiments would be valuable for investigating the genotypic and phenotypic contributions to variation in meristic characters, but such experiments are impractical in rattlesnakes.

Pattern characters showed a general pattern of geographic variation in which the northeastern, central-eastern, and north-central regions differed from the southern and western regions. Numbers of dorsal blotches and transverse elements (DMB, RDB, 
LDB, RVB, LVB, TE), as well as middorsal stripe width (MSW), had low values in the northeastern, central-eastern, and north-central regions and high values elsewhere. In contrast, band length and band spacing (BL, BS) as well as certain pattern anomalies (UB, YB) had high values in the northeastern, central-eastern, and north-central regions and low values elsewhere. A north-south pattern was present for tail crossbands (TCB). Unusual patterns were observed for two characters: body crossbands $(\mathrm{BCB})$ exhibited high values in northwestern and southeastern regions, and half bands (HB) had high values in northwestern regions and in Florida. Two characters did not accurately reflect variation in pattern. Low values of TCB in northern regions largely resulted from ontogenetic melanism of the tail, which seemingly obscured the caudal bands in many adult specimens. Higher values of MSW for many northern specimens apparently were an artifact of differential pigmentation and fading of the dorsum due to preservation. In such specimens, the pigmentation of the dorsolateral scales had numerous melanophores, whereas the scales near the middorsum had few or none. Thus, as the skin faded, a white, tan, or pale yellow middorsal stripe four to five scales wide would appear on some northern specimens. In contrast, the middorsal stripe of southern and western specimens tended to be reddish brown and three to four scales wide. Brown and Ernst (1986) reported fade-dependent middorsal stripes only in darker specimens. However, in this study such artifactual middorsal stripes were observed in both yellow and black color morphs.

The general pattern of body crossbands and blotches in Crotalus horridus appeared to be strongly influenced by latitude and elevation. Indeed, pattern may be even more strongly influenced by elevation than is scutellation. The Appalachian Mountains 
of the northeastern regions combine high latitude and high elevation, and consequently annual temperatures there are among the coldest in the species' range. The adaptive significance, if any, of lower numbers of body crossbands and blotches in the northeastern regions is not well understood. High numbers of dark pattern elements would be expected to increase the ability of a snake to thermoregulate at high elevations, but $C$. horridus displays the opposite trend. This geographic pattern may not be as maladaptive as it appears. The higher numbers of body crossbands, blotches, and scales in southern populations may all simply reflect the higher growth rates and larger body sizes attainable there. Furthermore, thermoregulatory function may be related more to variation in melanin density and ground color than to the pattern itself (see discussion of coloration, below).

Although the regressions among TE, BL, and BS only explained $15-40 \%$ of the total variation in pattern, all three of the regressions were significant. Additional regression analyses within selected study areas (not shown here) did not increase the total variation explained by each relationship, indicating that most of the unexplained variation was random, or at least not geographic. However, most of the explained variation was geographic, as demonstrated by patterns of those characters individually. The regressions of selected pattern characters did not provide a simple explanation for the adaptive significance of pattern variation, but they did expose some interesting relationships. In the northeastern regions, a lower number of transverse elements (TE) was associated with both longer band length (BL) and longer band spacing (BS). In fact, BL increased significantly with $\mathrm{BS}$ in the northeastern regions. This relationship appears counterintuitive, but BS was measured from the midlines of two consecutive bands, not 
from the edges of each band, in order to provide a measure of BS that was independent of BL. The interrelationships of TE, BL, and BS described here are likely to be important in understanding the genetic control of pattern formation in snakes.

Various mathematical models have been proposed to explain pattern formation in diverse organisms. Bard (1977) postulated that a reaction-diffusion model may account for the striping patterns of different species of zebras. He hypothesized that band spacing may be controlled by a simple mechanism that generates equally spaced vertical stripes, and that by acting at different times during embryonic development that mechanism could result in different band spacings in different species (Bard, 1977). Such a mechanism is thought to operate by irregularities in the concentration of a morphogen, that induces pattern formation (Turing, 1952). Differences in the wave pattern of the morphogen may produce a one-dimensional pattern of bands or stripes, or a twodimensional pattern of spots (Murray, 1981). Murray and Myerscough (1991) proposed a cell-chemotaxis mechanism for pattern formation in snakes. Their model involves physical and chemical interactions of chromatoblasts, that are able to produce their own chemoattractant, and explains some of the more complex patterns exhibited by ophidians. In contrast, pattern formation in butterfly wings is characterized by the evolution of compartmentalization of the wing surface, which has allowed pattern elements to gain independent genetic control (Nijhout, 1991, 2001).

Christman (1980) found a north-south pattern of variation in body crossbands and blotches in many Florida snakes and suggested that it may be related to mean annual temperature and differential gene flow. Even though the dynamics of peninsular populations are very different from continental populations, these factors may also be 
important in Crotalus horridus. However, geographic variation in pattern may also be strongly related to habitat. The disruptive pattern of $C$. horridus, often displayed by heavy-bodied ambush predators, may have evolved through natural selection for concealment on the forest floor (Klauber, 1972). Irregular band and blotch patterns may be especially adaptive for avoiding predation (Jackson et al., 1979). Conceivably, geographic variation in body crossbands and blotches may reflect habitat variation throughout eastern North America. Differences in habitat seem to have been a major factor in geographic variation in pattern for five subspecies of Agkistrodon contortrix (Van Devender and Conant, 1990). Dorsally constricted bands may have resulted from the movement of two subspecies (A. c. mokeson and A. c. contortrix) into temperate hardwood and southern pine forests in eastern North America (Van Devender and Conant, 1990). The effect of natural selection on pattern variation has been demonstrated in Nerodia sipedon from Lake Erie (Camin and Erlich, 1958; King, 1993; King and Lawson, 1997). Two color morphs are maintained by natural selection that favors unbanded morphs along rocky island shorelines and banded morphs along vegetated mainland shorelines. However, neither such distinct habitat differences nor such distinct pattern morphs were apparent across the range of $C$. horridus, so background pattern matching seems an unlikely explanation for differences in transverse elements, band length, and band spacing. 


\section{Analysis of Coloration}

Substantial geographic variation in coloration was observed in Crotalus horridus. Coloration is the most variable trait within and among different species of rattlesnakes (Gloyd, 1940; Klauber, 1972). Unfortunately, direct analysis of coloration was not possible due to the deleterious and highly variable effects of preservation, but most specimens retained at least enough color to be classified into broad categories. The maps of three characters as percentages of regional populations revealed a pattern of strong clinal variation among three broad areas of differentiation: the northeastern, centraleastern, and southern Appalachian regions, the northwestern regions, and the southern regions. The northeastern, central-eastern, and southern Appalachian regions had the highest incidences of high and melanistic melanin density (MD), dark and black ground color (GC), dark and black head color (HC), and absent or light postocular stripe (POS). All six of the melanistic specimens occurred in the northeastern and central-eastern regions. The northwestern regions were characterized by low $\mathrm{MD}$, dark $\mathrm{GC}$, dark $\mathrm{HC}$, and dark POS. The southern regions were characterized by low and medium MD, light to moderate GC, light to moderate $\mathrm{HC}$, and dark POS. However, all regions that were recognized on the basis of coloration overlapped each other broadly and formed a strong north-south cline in the eastern regions, as well as a weaker north-south cline in the western regions.

Integumentary color and reflectance are known to exert a strong influence on the thermal biology of snakes (Peterson et al., 1993). The higher melanin density and darker coloration of many Crotalus horridus in the northeastern regions south to southern 
Appalachian regions presumably enhance absorption of solar radiation. This would be consistent with the observation that darker individuals tend to occur in more densely forested and colder areas (Martin, 1992a). The northeastern through the southern Appalachian regions are characterized by relatively cool climates due to higher latitudes and/or elevations. Lower levels of melanin density in the northwestern regions appear to contradict Martin's observations. However, populations in the northwestern regions still exhibited darker coloration than those in the southern regions. The differences between the northeastern and northwestern regions may be due to the lower elevations and warmer summers of the northwestern regions, or they may simply reflect the independent phylogenetic history of $C$. horridus populations at the northeastern and northwestern extremes of the species' range (Clark et al., 2003). In any event, the persistence of yellow morphs in the northeastern regions is difficult to explain if selection for black pigmentation is so strong.

The greater frequency of black head color than black ground color in the northeastern regions may reflect the importance of the head in thermoregulation. The dorsal skin of the head is highly vascularized in Crotalus (A. Savitzky, unpublished data). Black pigmentation of the head may therefore maximize the rate of increase in body temperature during thermoregulation. Another rattlesnake species, Sistrurus catenatus, is known to thermoregulate by extending only the head into direct sunlight, while the body remains hidden in vegetation (T. Anton, personal communication). This behavior may reduce the snake's exposure to predation while still allowing the individual to thermoregulate. 


\section{Multivariate Analysis of Meristic Data}

To determine whether distinct geographic units exist within Crotalus horridus, a multivariate analysis was conducted to augment the analysis of individual characters. First, principal component analysis (PCA) was used to concentrate the total variation of each model into a smaller subset of variables and to identify characters important in explaining that variation. Then, cluster analysis was performed on the principal components to produce original classifications not based on any a priori groups. Each cluster analysis was tested using discriminant function analysis to determine how effectively the resulting groups could be identified and then compared to Gloyd's subspecies and Martin's regional morphs. Finally, the clusters of each model were mapped on a county map of the United States using a geographic information system.

Multivariate statistical analysis showed that patterns of variation within Crotalus horridus originated from the interaction of many variables. PCA indicated that band length (BL) was the most important character in this study. The next most important variables, in descending order, were band spacing (BS), the body blotch characters, transverse elements (TE), subcaudal scale count (CS), and the number of labial scales. The eigenvector structure of each PCA for males, females, and combined sexes was remarkably consistent. When males and females were analyzed separately, the main differences were that the labial counts rather than CS loaded high in PC3, and that CS and BS loaded high in PC4. This suggests that other sources of variation were strong enough to mask effectively the variation due to sexual dimorphism. The total variation in the sample presumably was composed of geographic, phylogenetic, and intraspecific 
genetic (polymorphic) variation, in addition to sexual dimorphism and any direct phenotypic effects imposed by the environment during ontogeny.

The most important characters used in previous studies to characterize geographic variation in Crotalus horridus differ from those identified in this study. In a previous analysis based upon individual characters, the most important characters in order of importance were ventrals (VS), subcaudals (CS), midbody dorsal scales (MDS), and transverse elements (TE) (Gloyd, 1940). Previous multivariate studies identified the most important characters, in order of importance, as CS, VS, and MDS (Pisani et al., 1973) and anterior dorsal scales (ADS) and VS (Brown and Ernst, 1986). The differences between previous studies and this one are due partly to the larger character set used in this study. The important characters of band length and spacing (BL, BS) were not quantified in previous studies. The complex patterns of crossbands and blotches were characterized by only one variable in each of the previous studies. In this study, however, VS, MDS, and ADS did not emerge as important characters in the PCAs. That lack of importance of VS was especially notable given its prominence in all previous studies. Because VS, CS, and BL exhibited significant sexual dimorphism, those variables should have loaded high in the PCA for combined sexes and low for males and females in the separate analyses. However, that was not the case. The high levels of variation of sexually dimorphic characters within each sex support the explanation that other sources of variation mask variation due to sexual dimorphism.

Cluster analysis was performed on the principal components of each model to produce an original classification for examining geographic variation in Crotalus horridus. Because the most important characters are not identified by the clustering 
algorithm in SAS, it was important to examine the cluster designations by sex and county to determine whether the procedure was clustering by sex in the combined-sex models. The clustering procedure did not create separate male and female clusters. The northeastern regions remained moderately distinct in all multivariate cluster analyses. The northwestern regions appeared very distinct in the three-, four-, and five-cluster models for females. The percentage maps effectively displayed the proportional frequency of clusters in each of the 32 regions. Those maps showed that the clusters in each model were not geographically exclusive, but displayed strong overlap and evidence of clinal variation.

\section{Analysis of Morphometric Data}

Strong sexual dimorphism was observed in all morphometric characters of Crotalus horridus, with males attaining larger sizes than females. Regression analysis explained significant variation in all ten relationships. Plots of the residuals showed that variances gradually increased with size in all regressions, but those effects were relatively weak and transformation of the data was not required. No other significant patterns were observed in the residuals. After removing the effect of size using snout-vent length (SVL), head length at the mandible (HLM), or mandible length (ML) as a covariate, strong sexual dimorphism was only observed for the regression of tail length (TL) on SVL. Even at the same SVL, males had significantly longer TL than females.

Significant sexual dimorphism was also detected in the regressions of head measurements on SVL due to the significant interaction between the covariate (SVL) and 
sex. However, the actual differences between males and females were extremely small. Significant differences were also detected in head length at the supraoccipital bone (HLS) on HLM, but again the differences were very small. The high number of significant relationships probably was influenced by the large sample used in this study. When the same regressions were performed on the combined data from the 12 study areas, the regressions of head measurements on SVL still had significant interactions with sex, but the effect of sex in the regression of HLS on HLM was not significant. In general, sexual dimorphism should be considered large for TL on SVL, but small or nonexistent for the remaining relationships.

Previous studies of Crotalus horridus found strong sexual size dimorphism (SSD) in tail and snout-vent lengths (Brown and Ernst, 1986; Galligan and Dunson, 1979 Gibbons, 1972; Gloyd, 1940). Males attain larger overall body sizes than females. In fact, most species in the genus Crotalus have male-larger SSD (Klauber, 1972). Colubrids that display male-larger SSD include the species complexes of Elaphe obsoleta (Burbrink, 2001) and Lampropeltis triangulum (Williams, 1978). In many snakes large body size in males occurs in larger species that engage in male-male combat (Shine, 1993), a behavior observed in C. horridus (Emst, 1992). Successful combat displays can increase mating success and are strongly dependent on body size in Vipera berus (Madsen et al., 1993) and probably in many other species as well. The correlation between SSD and male combat persists in snakes even after the effects of phylogeny are removed (Shine, 1994). Previous studies of $C$. horridus also found, by examining the ratio of TL to SVL, that males have relatively longer TL in relation to SVL. This relationship was displayed more effectively in this study by regressing TL on SVL. 
Sexual dimorphism in $\mathrm{TL}$ is thought to reflect a morphological constraint on tail size in males due to the housing of the hemipenes, selection for higher female fecundity through a proportional increase in body length, and sexual selection for male courtship success (King, 1989).

Substantial geographic variation within Crotalus horridus was observed clearly in all individual morphometric characters, which exhibited a general north-south pattern. Larger head and body sizes were observed in southern regions. Significant geographic variation also was observed by applying ANCOVA to the ten regressions of head and body measurements for males, females, and combined sexes. The resulting patterns of variation were more complex than the simple north-south pattern of individual characters. Although the effects of region were significant, the differences among regions probably were small because the regressions of each relationship were extremely tight and explained very high amounts of variation. Some relationships (for example, TL on SVL) could not be tested for regional effects because of significant interactions (SVL*region).

Regressions involving SVL were performed on males and females separately due to significant $\mathrm{SVL}^{*}$ sex interactions, but regressions involving HLM were performed on combined sexes for most characters, because only head width (HW) produced a significant $\mathrm{HLM}^{*}$ sex interaction. The most important ANCOVA results are summarized here. In males, head dimensions were largest in FL and NCCP and smallest in MO and KS after being adjusted for SVL. Specimens from LAS were grouped with northern and montane specimens that had smaller head dimensions. In females, head dimensions were largest in NCCP and NCPD after being adjusted for SVL, and the smallest head dimensions occurred for LAS, MO, KS, ILS, FL, and NCMT, with the regions varying 
according to the specific character. For the combined sexes, head dimensions were largest in NCCP, FL, and NCPD and smallest in MO, ILS, and KS after being adjusted for HLM. Again, LAS specimens were grouped with northern and montane specimens and had smaller head dimensions than the other southern regions. ANCOVA of QL on ML provided similar results to regressions of head dimensions on HLM. Considering the existence of such regional variation, it is remarkable that the factor analysis did not uncover greater geographic effects. Although factor analysis detected significant geographic variation in overall body size, it did not identify clear separations between regions, nor did it identify more specific shape factors.

Geographic variation in overall body size within Crotalus horridus also was observed in previous studies (Brown and Ernst, 1986; Gloyd, 1940). Specimens from southern regions were found to be significantly larger than those from northern regions. This general latitudinal pattern of variation in body size may be influenced by climatic factors in much the same way as suggested for scutellation, but to an even greater extent. For example, surface temperatures, length of the active season, humidity, and precipitation may have profound effects on annual growth, and ultimately, adult body size. Brattstrom $(1964,1967)$ proposed that ectotherms tend to follow the opposite of Bergmann's rule, which states that for endotherms body size increases with latitude. This trend presumably reflects the advantage of a relatively low surface-to-volume ratio for retaining metabolic heat. In contrast, only ectotherms with small body sizes (and greater surface area-to-volume ratios) would be able to reach operational body temperatures quickly enough at high latitudes and elevations (Brattstrom, 1967). Latitude and elevation appear to affect annual growth through differences in the thermal regime and 
length of the active season in Crotalus horridus (Martin, 1988, 1992a, 2002) and $C$. viridis (Diller and Wallace, 2002; Macartney et al., 1990). Body sizes of both species decrease dramatically with latitude. Hot summer climates are associated with large size in Thamnophis sirtalis (Rossman et al., 1996; Fitch, 1941) and T. ordinoides (Rossman et al., 1996; Fitch, 1940). However, some species only partially follow this latitudinal pattern in body size. In Lampropeltis triangulum, small body size occurs in the southern United States, but this pattern may be due to the cryptozoic habits of those populations (Williams, 1978). In any event, a recent study of the body size in Viperidae and Elapidae did not find support for Bergmann's rule (Reed, 2003).

The adaptive significance of geographic variation in head dimensions of Crotalus horridus is difficult to explain. No previous studies have demonstrated geographic variation in head measurements of $C$. horridus beyond the general north-south pattern of overall size. Although it was unexpected to find variation in head dimensions after adjusting head size for body and head length (SVL and HLM, respectively), the differences were small. Head measurements generally increased from north to south, but departures from that pattern were evident. Those departures may reflect allometric differences in cephalic growth and may be a consequence of genetic drift or of local adaptation to different frequencies of prey species. Phenotypic plasticity in head dimensions has been demonstrated for the Elaphe obsoleta complex (Burbrink, 2001), Nerodia rhombifera (Rossman, 1980), N. sipedon (Queral-Regil and King, 1998), and Bitis gabonica (Bonnet et al., 2001). However, tests of hypotheses concerning selection for specific head dimensions would require that prey sizes and frequencies within each region be known, which is beyond the scope of this study. 


\section{Geographic Variation}

The formal recognition of distinct geographic units within Crotalus horridus is not supported by this study, based upon three major findings. First, individual characters often showed discordant patterns of variation. Geographic transitions in character states from the northeastern region to the southern and western regions were highly variable. Some transitions were aligned north to south, some east to west, and some followed different patterns altogether. Furthermore, patterns of geographic variation in some sexually dimorphic characters were not concordant. For example, patterns of male ventral and subcaudal scales (VS, CS) were discordant with those of female VS and CS. Second, VS and midbody dorsal scales (MDS), important characters in previous schemes for mapping geographic variants, were not identified as important variables in any of the PCAs in this study. Differences in VS and MDS are often diagnostic for different species of rattlesnakes (Klauber, 1972), and Gloyd's early reliance on those characters to delineate subspecies of $\mathrm{C}$. horridus apparently led to their continued prominence in subspecies studies of the species. Third, the maps of all multivariate clusters showed extensive overlap, despite the general distinctiveness of specimens from the northeastern and Appalachian populations. Even in the two-cluster models, which had relatively low error rates and in which one cluster was largely limited to the northeastern regions, the second phenotypic cluster was not limited to southern and western regions, but was also spread widely across the northeastern regions. In fact, most clusters tended to be scattered to some degree over the entire distribution of the species. Therefore, division of 
Crotalus horridus into distinct geographic units, as proposed in some previous studies, is not justified by this study.

Gloyd $(1935,1940)$ divided Crotalus horridus into northern (C. h. horridus) and southern (C. h. atricaudatus) subspecies on the basis of univariate analyses of a few selected characters. Although this study supports to some degree a north-south pattern of geographic variation in C. horridus, the larger sample size and more extensive analytical tools used in this analysis revealed a more continuous pattern of geographic variation. In fact, Gloyd (1940) described how VS, CS, MDS, and transverse elements (TE) vary geographically within each subspecies, determined that the separation between the two subspecies is not sharply defined, and hypothesized that the zones of intergradation between his nominal subspecies may be fairly wide. Brown and Ernst (1986) found that anterior dorsal scales (ADS), MDS, posterior dorsal scales (PDS), and VS alone were insufficient to distinguish between $C . h$. horridus and $C$. h. atricaudatus, but the addition of adult size and middorsal stripe enabled them to distinguish the two subspecies. However, their study was based on small sample sizes and was limited to a small region of steep clinal variation in the eastern United States. Martin (1992) divided Crotalus horridus into three regional morphs: eastern, western, and southern. Although Martin's categories were effective for mapping geographic variation in coloration and pattern, the distribution of his three regional morphs does not coincide with either the univariate or multivariate analyses in this study. Moreover, separate discriminant function analyses applying Gloyd's subspecies and Martin's regional morphs to the large morphological data set assembled in this study did not support either model of geographic variation, even when testing males, females, and combined sexes in separate analyses. 
Evidence from two previous studies does not find support for the recognition of subspecies of Crotalus horridus. Pisani et al. (1973) conducted a multivariate analysis on a subset of Gloyd's data, initially trying to classify specimens from northeastern Kansas. Their analysis failed to support subspecies across the entire range of the species. However, their study was based on a relatively small sample size and tested only the $a$ priori nominal subspecies of Gloyd's classification. Although the study by Pisani et al. (1973) was not intended as a thorough analysis of geographic variation of C. horridus, their findings of high levels of intergradation between Gloyd's subspecies led to widespread rejection of formal subspecies until the latter were reinstated, with similar reservations, by Brown and Ernst (1986).

Most recently, Clark et al. (2003) analyzed the mtDNA sequence of the cytochrome b gene and found that 20 haplotypes occur within three shallow lineages. Two lineages exhibit north-south distributions, one along the east coast and one within the Mississippi River basin, although the two overlap extensively in the southeast. A third and much smaller lineage is found in western Missouri. Although the mtDNA results identify eastern and western lineages, the divergence between them is not deep enough and their distributions not sufficiently distinct to support the recognition of subspecies. More importantly, the eastern and western mtDNA lineages do not closely correspond to Martin's eastern and western morphs, nor to the fairly distinctive northeastern regions identified in this study. Both major mtDNA lineages overlap deeply within the range of Martin's southern morph. Furthermore, although the eastern mtDNA lineage aligns well in the north with the northeastern regions identified in this study, the lineage extends well beyond the southern boundary of the northeastern regions and into 
the southeastern Atlantic Coastal Plain and central Georgia. Therefore, the morphological and molecular data were in agreement that significant geographic variation exists within $C$. horridus, but neither data set supported the existence of distinct and exclusive geographic units within the species.

\section{Taxonomic Status}

Determination of the taxonomic status of an organism requires a theoretical framework for interpreting evolutionary processes, resolving speciation events, and applying species definitions. Although it is not my intention to discuss the philosophical issues underlying all species concepts, the question of what constitutes a species must be addressed because it is implicit in the delimitation of species and in the interpretation of geographic variation (Cracraft, 1989). The biological species concept (BSC) defined a species as groups of interbreeding populations that are reproductively isolated from other such groups (Mayr, 1942). However, its basic tenet of reproductive independence is not feasibly testable in most studies of geographic variation. Furthermore, reproductive compatibility is a pleisiomorphic character and thus inappropriate for application under current phylogenetic theory (Howard and Berlocher, 1998). Numerous species concepts have been advanced to replace the BSC and to operationalize species definitions for evolutionary studies. Different species concepts have been extensively reviewed in the systematics literature (Frost and Kluge, 1994; Howard and Berlocher, 1998; Otte and Endler, 1989) and their application to herpetological cases has been discussed at length (Frost and Hillis, 1990; Frost et al., 1992). 
Two species concepts have received the most attention recently. The evolutionary species concept (ESC) defines a species as a lineage of ancestral-descendant populations that has a separate evolutionary history from other lineages (first defined by Simpson, 1961; modified by Wiley, 1978, 1981; and further refined by Frost and Hillis, 1990): “A species is a single lineage of ancestral-descendant populations of organisms which maintains its identity from other such lineages and which has its own evolutionary tendencies and historical fate" (Wiley, 1978). The phylogenetic species concept (PSC) defines a species as the smallest group of ancestral-descendant populations, or lineages, diagnosable by one or more derived characters (Cracraft, 1983, 1989; Nelson and Platnick, 1981; Nixon and Wheeler, 1990; Rosen, 1978). Thus, "a species is the smallest diagnosable cluster of individual organisms within which there is a parental pattern of ancestry and descent" (Cracraft, 1983).

The ESC and PSC both succeed in reflecting the evolutionary history of a species while avoiding the operational and theoretical pitfalls of measuring reproductive compatibilities among different populations. An important component of the ESC is the identification of species as "largest evolving systems" (Frost and Hillis, 1990). In the ESC, species are real entities and are distinguished as monophyletic groups of populations by character analysis (Frost and Hillis, 1990). Two or more populations that have not completely differentiated would be classified as the same species. The major problem with the ESC is that determining the "evolutionary tendencies and historical fate" of a species can be ambiguous and may be difficult to operationalize for species delimitation. In contrast, the PSC identifies species as smallest evolving systems. In the PSC, species are also monophyletic and are equated with the terminal branches of 
phylogenetic trees. Two or more populations that have not completely differentiated may be classified as different species based on a single diagnostic character or unique combinations of characters. Thus, the PSC provides an operational definition because it explicitly defines species by using diagnostic character variation (Cracraft, 1983, 1989). However, the identification of species as smallest evolutionary units by the PSC may lead to the misclassification of populations exhibiting ephemeral or clinal variation (Frost and Hillis, 1990). Another problem of applying the PSC is that the number of recognized species is largely dependent on the resolving power of the character sets, analytical methods, and sampling densities used in different studies (Jorge da Silva and Sites, 1999).

For this study I prefer to apply the ESC because the identification of species as largest evolutionary units should improve the identification of biologically meaningful units. The ESC also allows additional information, such as the extent of gene flow and the existence of geographic barriers, to be considered together with character variation when defining species. However, like many species concepts the ESC and the PSC are not mutually exclusive, and the PSC can be treated as the operational form of the ESC (Frost and Kluge, 1994). Both have been defined as important components of a conceptual unification of species concepts, the general lineage species concept (de Queiroz, 1998). Given more comprehensive character sets, the ESC and PSC should agree in resolving the evolutionary history of a species.

Character-based species delimitation requires methodological decisions for dealing with polymorphism (Wiens, 1999). Species delimitation may be difficult because many species exist as regionally differentiated subpopulations that are connected by 
zones of intergradation (Endler, 1977). Species that manifest high levels of morphological variation can be identified as polytypic species and can be divided into distinct geographic subspecies (Mayr, 1942, 1963). The polytypic species concept may be useful for describing substantial and largely discontinuous regional variation, but assigning populations to different subspecies has been criticized for being too arbitrary and often biologically irrelevant (Cracraft, 1989; Frost and Hillis, 1990; Simpson, 1961; Wilson and Brown, 1953). In fact, many subpopulations probably can be determined as genetically distinct at some level, if enough sources of variation are examined. Thus, taxonomic classifications can only be made with reasonable objectivity down to the level of species. When traditional polytypic species are examined more closely, many subspecies are discovered to be real species (under the ESC or PSC) and can be elevated to species status (Frost and Hillis, 1990). However, subpopulations of widespread polymorphic species should be treated as regional morphs of the same species unless evidence for an independent phylogenetic history can be demonstrated.

Various standards for species delimitation in a manner consistent with the ESC have been proposed. Among the recommendations are that species be $100 \%$ recognizable by one or more single characters (Cracraft, 1989; Smith, 1990), 100\% recognizable by two or more concordant characters (Avise and Wollenberg, 1997), or recognizable by multivariate clusters (Sneath and Sokal, 1973). Multivariate statistical analyses are the most comprehensive of these methods when applied to morphological data, inasmuch as they rely upon multiple aspects of the phenotype. Cluster analysis has been used to examine the intergrade zone between Crotalus viridis oreganus and $C . v$. helleri (Schneider, 1986), the specific status of Pituophis melanoleucus ruthveni 
(Reichling, 1995), and intergradation within Sceloporus variabilis (Sites and Dixon, 1982). Canonical variate analysis has been used to resolve the species complexes of Daboia russelli (Wüster et al., 1992), Bothrops atrox (Wüster et al., 1996), Elaphe obsoleta (Burbrink, 2001), Micrurus frontalis (Jorge da Silva and Sites, 1999), and Naja naja (Wüster and Thorpe, 1992). A specific standard has not been explicitly stated for multivariate statistical analyses, although complete separation of clusters certainly implies different species. A more practical standard might be $95-100 \%$ separation between multivariate clusters and complete separation between the standard deviations of each cluster. That practical standard might also assign specific status to populations verified as distinct by $95-100 \%$ correct crossvalidation in discriminant function analysis.

The results of this study indicate that, under either the ESC or PSC, Crotalus horridus cannot be divided into separate taxa corresponding to Gloyd's (1940) subspecies, Martin's (1992) regional morphs, or any other system of classification. The discordant patterns of variation among individual characters, the overlapping patterns of coloration, and the extensive overlap among the multivariate clusters collectively indicate that the zones of intergradation (sensu Endler, 1977, and thus not implying subspecific status) among subpopulations of $C$. horridus are much broader than previously thought. Furthermore, the general patterns of geographic variation are strongly clinal and support the conclusion that $C$. horridus is a single widespread, polymorphic species. The multivariate approach detected fine-grained geographic structure that had been partially hidden in analyses of individual characters. However, in this analysis the cluster percentage maps revealed a pattern of geographic variation consisting of two strong north-south clines extending southward from the northeastern and northwestern regions. 
Pisani et al. (1973) concluded that their characters show smooth clinal variation between northern and southern groups. Significantly, Clark et al. (2003) detected two mtDNA lineages that overlap in the southern regions and extend separately into the northwestern and northeastern regions, following the same general paths as the axes of clinal variation in this study.

Studies of other reptiles have demonstrated that early classifications based on a few color pattern characters or single morphological characters often do not agree with more comprehensive molecular and morphological analyses. Some representative ophidian taxa that have been subjected to recent re-analysis include the Morelia amethistina complex (Harvey et al., 2000), the Elaphe guttata complex (Burbrink, 2002), the Elaphe obsoleta complex (Burbrink, 2001; Burbrink et al., 2000), Lampropeltis zonata (Rodríguez-Robles et al., 1999), the Pituophis complex (Reichling, 1995; Rodríguez-Robles and de Jesús-Escobar, 2000), the Bothrops complex (Puorto et al., 2001; Wüster et al., 1996, 1999), the Crotalus viridis complex (Douglas et al., 2002; Pook et al., 2000), Daboia russelli (Wüster et al., 1992), Lachesis (Zamudio and Greene, 1997), the Micrurus frontalis complex (Jorge da Silva and Sites, 1999), and the Naja naja complex (Wüster and Thorpe, 1992). Some representative saurian taxa include Phrynosoma (Reeder and Montanucci, 2001), Phrynosoma douglasi (Zamudio et al., 1997), Sceloporus (Wiens and Reeder, 1997), and Sceloporus undulatus (Miles et al., 2002). In many cases, previous subspecies have been elevated to full species. In other groups, new taxa have been described based on newly analyzed patterns of geographic variation. In contrast, Crotalus horridus remains a widespread, polymorphic species, the subpopulations of which have not differentiated into separate taxa. 


\section{Historical Zoogeography}

Crotalus horridus may have diverged in the middle Miocene, when caenophidians were experiencing extensive radiations in response to climatic change and increased habitat diversity (Holman, 1977; Van Devender and Conant, 1990). During a period of global warming 18-13 Ma, in the early and middle Miocene, broad-leaved evergreen vegetation and coniferous forest encroached slightly on deciduous forest (Potts and Behrensmeyer, 1992). A period of global cooling began about $13 \mathrm{Ma}$ and continued through the Pliocene, resulting in the retreat of the paleotropical flora and expansion of deciduous forests, grasslands, and conifers. The evolutionary diversification of caenophidians may also be linked to the middle and late Cenozoic radiation of small mammals (Carroll, 1988; Savitzky, 1980). The oldest fossils of $C$. horridus date from the early and late Pliocene (Holman, 1979), and the species was widely distributed throughout eastern North America during the Pleistocene (Holman, 1995).

Several authors (Brattstrom, 1964; Foote and MacMahon, 1977; Gloyd, 1940) have placed Crotalus horridus within the $C$. durissus group, which consists largely of species that inhabit arid habitats in southwestern North America, Central America, and South America. Thus, the ancestor of $C$. horridus was probably a xeric-adapted species in southwestern North America that dispersed northeastward, adapting to the humid conditions of deciduous forests of eastern North America, eventually reaching high latitudes and elevations. However, recent molecular evidence places $C$. horridus in a lineage with $C$. viridis and $C$. scutulatus (Murphy et al., 2002). If that phylogenetic hypothesis is correct, the ancestor of $C$. horridus may either have dispersed eastward 
from the Great Plains or from xeric habitats in the southwest as described above. The fossil record is not sufficiently complete to reveal much of the pre-Pleistocene history of C. horridus. However, the Pliocene is characterized as a warm epoch, during which temperatures did not fall below those of the Pleistocene interglacials, even at higher latitudes (Matthews and Poore, 1981).

The Pleistocene glaciations had profound effects on the present distributions of amphibians and reptiles. The initial Pleistocene cooling episode began globally 2.4-2.5 Ma (Morrison, 1991; Riser, 2001). Terrestrial vertebrate communities were subjected to 40-50 cyclic glaciations of uneven distribution and varying magnitude (Morrison, 1991; Riser, 2001). Whereas glacial phases typically lasted $100 \mathrm{Ka}$, interglacial phases lasted only about $20 \mathrm{Ka}$ (Delcourt and Delcourt, 1993). During glacial maxima, previously northern amphibians and reptiles were restricted to southern regions, but most southern species did not move farther south than their present ranges (Holman, 1995). The sympatry of extralimital northern and southern species has been explained by the Pleistocene climatic equability hypothesis: cooler summers provided conditions favorable to northern species and warmer winters provided favorable conditions for southern species (Graham and Lundelius, 1984; Holman, 1976, 1995; Lundelius et al., 1983).

The Wisconsin glacial maximum is dated at $17-21 \mathrm{Ka}$ (Riser, 2001). At its maximal extent the Laurentian ice cap covered half of lowa, Illinois, and Indiana, most of northern and western Ohio, northwestern and northeastern Pennsylvania, northern New Jersey, and Long Island (Ross, 1965; Delcourt and Delcourt, 1987). However, a significant unglaciated region (the Driftless Area) extended from the northwestern corner of Illinois northward along the Mississippi River and into the southwestern quadrant of 
Wisconsin. Climatic conditions limited herpetofaunal species adapted to temperate conditions to areas south of an ecotone positioned along $32^{\circ} \mathrm{N}$ latitude (Delcourt and Delcourt, 1987). Whereas boreal vegetation covered regions north of the ecotone, temperate vegetation dominated below it. Rapid climatic warming began at $14.8 \mathrm{Ka}$, causing the glacial ice sheets to retreat (Holman, 1995; Riser, 2001). Glacial retreat lasted until $8 \mathrm{Ka}$ and roughly coincided with a period of unusual warming from 9-5 Ka (Delcourt and Delcourt, 1991). Climatic warming and glacial retreat allowed temperate vegetation and many herpetological species to reinvade more northern regions of North America.

Presumably Crotalus horridus expanded its range northward from southern refugia after the last glacial maximum. The north-south patterns of clinal variation detected in this study apparently reflect this northward expansion of the species. Although other patterns of clinal variation could be hypothesized, alternative patterns of character variation (for example, east-west) are not as steep as the north-south clines. The loss of genetic diversity with increasing latitude also supports this northward expansion (Clark et al., 2003).

Gloyd (1940) suggested that the apparent convergence of his northern and southern subspecies in several diagnostic characters (ventrals, subcaudals, and dorsal scale rows) within the southwestern regions indicates that this area is the center from which Pleistocene populations dispersed northeastward in the Holocene. Although patterns of variation in some characters in this study (female subcaudals, transverse elements, and coloration of the postocular stripe) exhibit a northeast-southwest cline, most of the results support two separate north-south clines. Moreover, zones of 
intergradation do not necessarily reveal centers of dispersal. There is no climatic evidence to suggest that $C$. horridus was limited only to southwestern refugia during the Wisconsin glaciation. In fact, molecular data show that the highest diversity of haplotypes occurs in Georgia, suggesting that the primary Pleistocene refugia may have been located in the southeast, south of the Appalachian Mountains (Clark et al., 2003). Indeed, during the Wisconsin glacial maximum, $C$. horridus may have occurred throughout southern temperate forests from the southern Atlantic Coastal Plain to southeastern Texas. Upon subsequent warming, populations expanded northward, where they may have experienced strong selection due to the harsher climates of the higher latitudes and the Appalachian Mountains.

The arrival of Crotalus horridus in the Pine Barrens of southern New Jersey is enigmatic. Those populations are relatively isolated from other eastern populations. Most previous authors considered that the populations of the Pine Barrens were intermediate between a northern subspecies inhabiting the northeastern regions and a southern subspecies from the southeastern Coastal Plain, mostly due to the bold reddishbrown middorsal stripe of many specimens (Conant, 1998; Gloyd, 1940; Martin, 1992a). Pine Barrens populations may have been derived by either northern forms invading from the northwest through stream corridors or southern forms from the southeastern Coastal Plain (Conant, 1998). Martin (1992a) suggested that southeastern lowland populations dispersed as far north as Long Island during the climatic warming trend about $8 \mathrm{Ka}$, and interbred with northeastern upland populations in the Pine Barrens. The morphological data examined in this study provide little support for the close affinity between Pine Barrens and southeastern Coastal Plain populations. Although the higher number of body 
crossbands for females in the Pine Barrens is consistent with clinal variation within southern populations, most individual characters (such as the number of dorsal scales, ventral scales, subcaudal scales, labial scales, transverse elements, band length, and band spacing), as well as all cluster analyses, strongly associate Pine Barrens populations with other northeastern populations. (Failure to recognize a middorsal stripe in most southern New Jersey specimens in this study was due largely to fading of the pigmentation.) Thus, populations from the Pine Barrens probably were derived mostly from northeastern upland populations, possibly with limited genetic influence from some southern migrants. The relatively higher frequencies of $\mathrm{Y}$-bands and U-bands in the northeastern regions may be due to inbreeding depression. The use of communal denning sites in montane habitats may increase the frequency of mating within those populations and may explain the reduced genetic variation of those populations (Bushar et al., 1998). It is noteworthy that pattern irregularities may reflect a phenotypic manifestation of that limited genotypic variation.

Many ophidian taxa with similar geographic distributions in eastern North America have actually differentiated to a greater extent than has Crotalus horridus. Such taxa include Agkistrodon contortrix (Gloyd and Conant, 1990), the Elaphe obsoleta complex (Burbrink, 2001; Burbrink et al., 2000), Lampropeltis getula (Dessaur and Pough, 1975), L. triangulum (Williams, 1978), and Thamnophis sirtalis (Rossman et al., 1996). The lower Mississippi River drainage and the Appalachian Mountains constitute significant geographic barriers to many species (Rossman, 1962, 1963; Blair, 1965). For example, east-west partitioning of the Elaphe obsoleta complex shows four separate clades largely influenced by those geographic barriers (Burbrink, 2001; Burbrink et al., 
2000). Molecular data reveal an east-west separation within C. horridus corresponding to the Appalachian Mountains, although the genetic divergences are not deep and some haplotypes are shared among regions (Clark et al., 2003). The morphological data examined in this study did not show any indication of east-west partitioning of $C$. horridus across the Appalachians. The fact that $C$. horridus exhibits so little differentiation may be due to the large seasonal movements made by male individuals, compared to the lower vagility of many other species (Brown et al., 1982; Reinert and Zappalorti, 1988). In addition, C. horridus can tolerate high elevations, inhabit extensive wetlands, and regularly traverse large rivers (Martin, 1992a, 2002; A. Savitzky and C. Petersen, unpublished data). These aspects of their biology may have supported sufficient gene flow among different populations to maintain the species as a single evolutionary lineage. However, anthropogenic habitat fragmentation, coupled with declining sizes of many populations, may adversely affect metapopulation dynamics and gene flow in the future. Already most northeastern populations of Crotalus horridus that existed at the onset of European colonization have been extirpated (Martin, 1992a).

It should also be emphasized that, although this study fails to support the recognition of formal subspecies, the northeastern and Appalachian populations do emerge as the most distinctive major geographic unit within Crotalus horridus. Those populations, which are especially vulnerable due to their low reproductive cycle, late age of first reproduction, communal hibernation behavior, and frequent occurrence near major urban centers (Brown, 1991, 1993; Martin, 1993, 2002), may constitute a "distinct population segment" for the purposes of the United States Endangered Species Act of 1973 (United States Fish and Wildlife Service, 1999). 


\section{CONCLUSIONS}

Morphological variation within Crotalus horridus is more complex than demonstrated by previous studies. Sexual dimorphism is strong in ventral and subcaudal scales, and weak to moderate in band length and band spacing. Substantial geographic variation within $C$. horridus was observed in all meristic characters. Scutellation and morphometric characters show a general north-south pattern of geographic variation for most characters. Pattern characters show a general pattern of geographic variation in which the northeastern, central-eastern, and north-central regions differ from the southern and western regions. Coloration displays strong clinal variation among the combined northeastern, central-eastern, and southern Appalachian regions, the northwestern regions, and the southern regions.

Principal component analysis indicated that band length is the most important variable for characterizing geographic variation. The northeastern regions remained moderately distinct in all multivariate cluster analyses. However, the clusters in all models showed extensive geographic overlap. Cluster analysis revealed two north-south patterns of clinal variation among the northeastern, northwestern, and southern regions. Discordant patterns of variation among individual characters, overlapping patterns of coloration, and extensive overlap among the multivariate clusters collectively indicate that zones of intergradation among subpopulations of $C$. horridus are much broader than previously thought. Furthermore, the general patterns of geographic variation are strongly clinal and support the conclusion that $C$. horridus is a single widespread, polymorphic species within which formal subspecies should not be recognized. 


\section{LITERATURE CITED}

Ahrens, C. D. 1994. Meteorology Today: An Introduction to Weather, Climate, and the Environment, 5th edition. West Publishing Company, Saint Paul, Minnesota.

Aldridge, R. D., and W. S. Brown. 1995. Male reproductive cycle, age at maturity, and cost of reproduction in the timber rattlesnake (Crotalus horridus). Journal of Herpetology 29:399-407.

Avise, J. C., and K. Wollenberg. 1997. Phylogenetics and the origin of species. Proceedings of the National Academy of Sciences USA 94:7748-7755.

Bard, J. B. L. 1977. A unity underlying the different zebra striping patterns. Journal of Zoology London 183:527-539.

Barry, R. C., and R. J. Chorley. 1998. Atmosphere, Weather, and Climate, 7th edition. Routledge, New York.

Blair, W. F. 1965. Amphibian speciation. Pp. 543-556. In H. E. Wright, Jr., and D. G. Frey (Eds.), The Quaternary of the United States: A Review Volume for the VII Congress of the International Association for Quaternary Research. Princeton University Press, Princeton, New Jersey.

Blaney, R. M. 1971. Systematics of the common kingsnake, Lampropeltis getulus. Ph.D. Dissertation, Louisiana State University, Baton Rouge, Louisiana.

Bonnet, X. R. Shine, G. Naulleau, and C. Thiburce. 2001. Plastic vipers: Influence of food intake on the size and shape of Gaboon vipers (Bitis gabonica). Journal of Zoology 255:341-351.

Brattstrom, B. H. 1954. The fossil pit-vipers (Reptilia: Crotalidae) of North America. Transactions of the San Diego Society of Natural History 12:31-46.

Brattstrom, B. H. 1958. New records of Cenozoic amphibians and reptiles from California. Bulletin of the Southern California Academy of Sciences 57:5-13.

Brattstrom, B. H. 1964. Evolution of the pit vipers. Transactions of the San Diego Society of Natural History 13:185-268.

Brattstrom, B. H. 1965. Body temperatures of reptiles. American Midland Naturalist 73:376-422.

Brattstrom, B. H. 1967. A succession of Pliocene and Pleistocene snake faunas from the high plains of the United States. Copeia 1967:188-202. 
Brown, C. W., and C. H. Ernst. 1986. A study of variation of eastern timber rattlesnakes, Crotalus horridus Linnaeus (Serpentes: Viperidae). Brimleyana 12:57-74.

Brown, W. S. 1991. Female reproductive ecology in a northern population of the timber rattlesnake, Crotalus horridus. Herpetologica 47:101-115.

Brown, W. S. 1993. Biology, Status, and Management of the Timber Rattlesnake (Crotalus horridus): A Guide for Conservation. Society for the Study of Amphibians and Reptiles, Herpetological Circular 22:1-78.

Brown, W. S., D. W. Pyle, K. R. Greene, and J. B. Friedlaender. 1982. Movements and temperature relationships of timber rattlesnakes (Crotalus horridus) in northeastern New York. Journal of Herpetology 16:151-161.

Burbrink, F. T. 2001. Systematics of the eastern ratsnake complex (Elaphe obsoleta). Herpetological Monographs 15:1-53.

Burbrink, F. T. 2002. Phylogeographic analysis of the cornsnake (Elaphe guttata) complex as inferred from maximum likelihood and Bayesian analyses. Molecular Phylogenetics and Evolution 25:465-476.

Burbrink, F. T., R. Lawson, and J. B. Slowinski. 2000. Mitochondrial DNA phylogeography of the North American rat snake (Elaphe obsoleta): A critique of the subspecies concept. Evolution 54:2107-2114.

Bushar, L. M., H. K. Reinert, and L. Gelbert. 1998. Genetic variation and gene flow within and between local populations of the timber rattlesnake, Crotalus horridus. Copeia 1998:411-422.

Cadle, J. E. 1987. Geographic distribution: Problems in phylogeny and zoogeography. Pp. 77-105. In R. A. Seigel, J. T. Collins, and S. S. Novak (Eds.), Snakes: Ecology and Evolutionary Biology. Macmillan Publishing Company, New York.

Camin, J. H., and P. R. Erlich. 1958. Natural selection in water snakes (Natrix sipedon L.) on islands in Lake Erie. Evolution 12:504-511.

Campbell, J. A., and W. W. Lamar. 1989. The Venomous Reptiles of Latin America. Comstock Publishing Associates, Cornell University Press, Ithaca, New York.

Carroll, R. L. 1988. Vertebrate Paleontology and Evolution. W. H. Freeman and Company, New York.

Christman, S. P. 1980. Patterns of geographic variation in Florida snakes. Bulletin of the Florida State Museum, Biological Sciences 25:157-256. 
Clark, A. M., P. E. Moler, E. E. Possardt, A. H. Savitzky, W. S. Brown, and B. W. Bowen. 2003. Phylogeography of the timber rattlesnake (Crotalus horridus) based on mtDNA sequences. Journal of Herpetology 37:145-154.

Conant, R. 1990. The fossil history of the genus Agkistrodon in North America. Pp. 539-543. In H. K. Gloyd and R. Conant, Snakes of the Agkistrodon Complex: A Monographic Review. Society for the Study of Amphibians and Reptiles, Contributions to Herpetology 6:1-614.

Conant, R. 1998. A zoogeographical review of the amphibians and reptiles of southern New Jersey, with emphasis on the Pine Barrens. Pp. 467-488. In R. T. T. Forman (Ed.), Pine Barrens: Ecosystem and Landscape. Rutgers University Press, New Brunswick, New Jersey.

Conant, R., and J. T. Collins. 1991. A Field Guide to Reptiles and Amphibians: Eastern/Central North America, 3rd edition. Houghton Mifflin Company, Boston, Massachusetts.

Cracraft, J. 1983. Species concepts and speciation analysis. Pp. 159-187. In R. F. Johnston (Ed.), Current Ornithology, Volume 1. Plenum Press, New York.

Cracraft, J. 1989. Speciation and its ontology: The empirical consequences of alternative species concepts for understanding patterns and processes of differentiation. Pp. 28-59. In D. Otte and J. A. Endler (Eds.), Speciation and its Consequences. Sinauer Associates, Sunderland, Massachusetts.

Crother, B. I., J. A. Campbell, and D. M. Hillis. 1992. Phylogeny and historical biogeography of the palm-vipers, genus Bothriechis: Biochemical and morphological evidence. Pp. 1-19. In J. A. Campbell and E. D. Brodie, Jr. (Eds.), Biology of the Pitvipers. Selva Press, Tyler, Texas.

Cundall, D. 2002. Envenomation strategies, head form, and feeding ecology in vipers. Pp. 149-161. In G. W. Schuett, M. Hoggren, M. E. Douglas, and H. W. Greene (Eds.), Biology of the Vipers. Eagle Mountain Publishing, Eagle Mountain, Utah.

Darlington, P. J. 1957. Zoogeography: The Geographical Distribution of Animals. John Wiley and Sons, New York.

Delcourt, H. R., and P. A. Delcourt. 1991. Quaternary Ecology: A Paleoecological Perspective. Chapman and Hall, New York.

Delcourt, P. A., and H. R. Delcourt. 1987. Long-Term Forest Dynamics of the Temperate Zone: A Case Study of Late-Quaternary Forests in Eastern North America, Ecological Studies 63. Springer-Verlag, New York. 
Delcourt, P. A., and H. R. Delcourt. 1993. Paleoclimates, paleovegetation, and paleofloras during the Late Quaternary. Pp. 71-94. In Flora of North America Editorial Committee (Eds.), Flora of North America, North of Mexico. Oxford University Press, New York.

de Queiroz, K. 1998. The general lineage concept of species, species criteria, and the process of speciation: A conceptual unification and terminological recommendations. Pp. 57-75. In D. J. Howard and S. H. Berlocher (Eds.), Endless Forms. Oxford University Press, New York.

Dessaur, H. C., and F. H. Pough. 1975. Geographic variation of blood proteins and the systematics of kingsnakes (Lampropeltis getulus). Comparative Biochemistry and Physiology 50B:9-12.

Diller, L. V., and R. L. Wallace. 2002. Growth, reproduction, and survival in a population of Crotalus viridis oreganus in north central Idaho. Herpetological Monographs 16:26-45.

Douglas, M. E., M. R. Douglas, G. W. Schuett, L. W. Porras, and A. T. Holycross. 2002. Phylogeography of the western rattlesnake (Crotalus viridis) complex, with emphasis on the Colorado Plateau. Pp. 11-50. In G. W. Schuett, M. Hoggren, M. E. Douglas, and H. W. Greene (Eds.), Biology of the Vipers. Eagle Mountain Publishing, Eagle Mountain, Utah.

Endler, J. A. 1977. Geographic Variation, Speciation, and Clines. Princeton University Press, Princeton, New Jersey.

Ernst, C. H. 1989. Snakes of Eastern North America. George Mason University Press, Fairfax, Virginia.

Ernst, C. H. 1992. The Venomous Snakes of North America. Smithsonian Institution Press, Washington D. C.

ESRI, 1992-1999. ArcView, version 3.2. Environmental Systems Research Institute, Redlands, California.

ESRI, 1997. ArcUSA 1:2M: A Comprehensive GIS Database for Use with ARC/INFO and ArcView, 1st edition. Environmental Systems Research Institute, Redlands, California.

Everitt, B. S., S. Landau, and M. Leese. 2001. Cluster Analysis, 4th edition. Oxford University Press, New York. 
Fitch, H. S. 1940. Geographic variation in garter snakes of the species Thamnophis sirtalis in the Pacific Coast region of North America. American Midland Naturalist 26:570-592.

Fitch, H. S. 1941. A biogeographical study of the ordinoides artenkreis of garter snakes (genus Thamnophis). University of California Publications in Zoology 44:1-150.

Foote, R., and J. A. MacMahon. 1977. Electrophoretic studies on rattlesnake (Crotalus and Sistrurus) venom: Taxonomic implications. Journal of Biochemistry and Physiology 57B:235-241.

Fox, W. W., C. Gordon, and M. H. Fox. 1961. Morphological effects of low temperatures during the embryonic development of the garter snake, Thamnophis elegans. Zoologica 46:57-71.

Frost, D. R., and D. M. Hillis. 1990. Species in concept and practice: Herpetological applications. Herpetologica 46:87-104.

Frost, D. R., and A. G. Kluge. 1994. A consideration of epistemology in systematic biology, with special reference to species. Cladistics 10:259-294.

Frost, D. R., A. G. Kluge, and D. M. Hillis. 1992. Species in contemporary herpetology: Comments on phylogenetic inference and taxonomy. Herpetological Review 23:46-54.

Galligan, J. H., and W. A. Dunson. 1979. Biology and status of timber rattlesnake (Crotalus horridus) populations in Pennsylvania. Biological Conservation 15:1358.

Gibbons, J. W. 1972. Reproduction, growth, and sexual dimorphism in the canebrake rattlesnake (Crotalus horridus atricaudatus). Copeia 1972:222-226.

Gloyd, H. K. 1935. The canebrake rattlesnake. Copeia 1935:175-178.

Gloyd, H. K. 1940. The rattlesnakes, genera Sistrurus and Crotalus. Chicago Academy of Sciences Special Publications 4:1-266.

Gloyd, H. K., and R. Conant. 1990. Snakes of the Agkistrodon Complex: A Monographic Review. Society for the Study of Amphibians and Reptiles, Contributions to Herpetology 6:1-614.

Gould, S. J., and R. F. Johnston. 1972. Geographic variation. Annual Review of Ecology and Systematics 3:457-498. 
Gower, J. C. 1967. A comparison of some methods of cluster analysis. Biometrics 23:623-637.

Graham, R. W., and E. L. Lundelius, Jr. 1984. Coevolutionary disequilibrium and Pleistocene extinctions. Pp. 223-249. In P. S. Martin and R. G. Klein (Eds.), Quaternary Extinctions: A Prehistoric Revolution. University of Arizona Press, Tucson, Arizona.

Greene, H. W. 1997. Snakes: The Evolution of Mystery in Nature. University of California Press, Berkeley and Los Angeles, California.

Grismer, L. L., J. A. McGuire, and B. D. Hollingsworth. 1994. A report on the herpetofauna of the Vizcaíno Peninsula, Baja California, México with a discussion of its biogeographic and taxonomic implications. Bulletin of the Southern California Academy of Sciences 93:45-80.

Gutberlet, Jr., R. L., and M. B. Harvey. 2002. Phylogenetic relationships of New World pitvipers as inferred from anatomical evidence. Pp. 51-68. In G. W. Schuett, M. Hoggren, M. E. Douglas, and H. W. Greene (Eds.), Biology of the Vipers. Eagle Mountain Publishing, Eagle Mountain, Utah.

Harvey, M. B., D. G. Barker, L. K. Ammerman, and P. T. Chippindale. 2000.

Systematics of pythons of the Morelia amethistina complex (Serpentes: Boidae) with the description of three new species. Herpetological Monographs 14:139185.

Holman, J. A. 1976. Paleoclimatic implications of "ecologically incompatible" herpetological species (late Pleistocene: southeastern United States). Herpetologica 32:290-295.

Holman, J. A. 1977. Upper Miocene snakes (Reptilia, Serpentes) from southeastern Nebraska. Journal of Herpetology 11:323-335.

Holman, J. A. 1979. A review of North American Tertiary snakes. Publications of the Museum, Michigan State University, Paleontological Series 1:203-206.

Holman, J. A. 1981. A herpetofauna from an eastern extension of the Harrison Formation (Early Miocene: Arikareean), Cherry County, Nebraska. Journal of Vertebrate Paleontology 1:49-56.

Holman, J. A. 1991. North American Pleistocene herpetofaunal stability and its impact on the interpretation of modern herpetofaunas: An overview. Illinois State Museum Scientific Papers 23:227-235. 
Holman, J. A. 1995. Pleistocene Amphibians and Reptiles in North America. Oxford University Press, New York.

Howard, D. J., and S. H. Berlocher. 1998. Endless Forms. Oxford University Press, New York.

Jackson, J. F., W. Ingram, III, and H. W. Campbell. 1976. The dorsal pigmentation pattern of snakes as an antipredator strategy: A multivariate approach. American Naturalist 110:1029-1053.

Jorge da Silva, Jr., N., and J. W. Sites, Jr. 1999. Revision of the Micrurus frontalis complex (Serpentes: Elapidae). Herpetological Monographs 13:142-194.

Keenlyne, K. D. 1978. Reproductive cycles in two species of rattlesnakes. American Midland Naturalist 100:368-375.

Khattree, R., and D. N. Naik. 2000. Multivariate Data Reduction and Discrimination with SAS Software. SAS Institute, Cary, North Carolina.

King, R. B. 1989. Sexual dimorphism in snake tail length: Sexual selection, natural selection, or morphological constraint? Biological Journal of the Linnean Society 38:133-154.

King, R. B. 1993. Color-pattern variation in Lake Erie water snakes: Prediction and measurement of natural selection. Evolution 47:1819-1833.

King, R. B., and R. Lawson. 1997. Microevolution in island water snakes: What processes influence color pattern differences in Lake Erie island and mainland water snakes? Bioscience 47:279-286.

Klauber, L. M. 1956. Rattlesnakes: Their Habits, Life Histories, and Influences on Mankind, Volumes 1 and 2. University of California Press, Berkeley and Los Angeles, California.

Klauber, L. M. 1972. Rattlesnakes: Their Habits, Life Histories, and Influences on Mankind, Volumes 1 and 2, 2nd edition. University of California Press, Berkeley and Los Angeles, California.

Knight, A., L. D. Densmore, III, and E. D. Rael. 1992. Molecular systematics of the Agkistrodon Complex. Pp. 49-69. In J. A. Campbell and E. D. Brodie, Jr. (Eds.), Biology of the Pitvipers. Selva Press, Tyler, Texas.

Knight, A., D. Styler, S. Pelikan, J. A. Campbell, L. D. Densmore, III, and D. P. Mindell. 1993. Choosing among hypotheses of rattlesnake phylogeny: A best-fit rate test for DNA sequence data. Systematic Biology 42:356-367. 
Kraus, F., D. G. Mink, and W. M. Brown. 1996. Crotaline intergeneric relationships based on mitochondrial DNA sequence data. Copiea 1996:763-773.

Leviton, A. E., R. H. Gibbs, Jr., E. Heal, and C. E. Dawson. 1985. Standards in herpetology and ichthyology: Part 1. Standard symbolic codes for institutional resource collections in herpetology and ichthyology. Copeia 1985:802-832.

Lundelius E. L., R. W. Graham, E. Anderson, J. Guilday, J. A. Holman, D. W. Steadman, and S. D. Webb. 1983. Terrestrial vertebrate faunas. Pp. 311-353. In S. C. Porter (Ed.), Late Quaternary Environments of the United States, Volume 1, The Late Pleistocene. University of Minnesota Press, Minneapolis, Minnesota.

Macartney, J. M., P. T. Gregory, and M. B. Charland. 1990. Growth and sexual maturity of the western rattlesnake, Crotalus viridis, in British Columbia. Copeia 1990:528-542.

Madsen, T., R. Shine, J. Loman, and T. Haakansson. 1993. Determinants of mating success in male adders, Vipera berus. Animal Behaviour 45:491-499.

Martin, W. H. 1988. Life history of the timber rattlesnake. Catesbeiana 8:9-12.

Martin, W. H. 1992a. The timber rattlesnake: Its distribution and natural history. Pp. 13-22. In T. F. Tyning (Ed.), Conservation of the Timber Rattlesnake in the Northeast. Massachusetts Audubon Society, Lincoln, Massachusetts.

Martin, W. H. 1992b. Phenology of the timber rattlesnake (Crotalus horridus) in an unglaciated section of the Appalachian Mountains. Pp. 259-277. In J. A. Campbell and E. D. Brodie, Jr. (Eds.), Biology of the Pitvipers. Selva Press, Tyler, Texas.

Martin, W. H. 1993. Reproduction of the timber rattlesnake (Crotalus horridus) in the Appalachian mountains. Journal of Herpetology 27:133-143.

Martin, W. H. 2002. Life history constraints on the timber rattlesnake (Crotalus horridus) at its climatic limits. Pp. 285-306. In G. W. Schuett, M. Hoggren, M. E. Douglas, and H. W. Greene (Eds.), Biology of the Vipers. Eagle Mountain Publishing, Eagle Mountain, Utah.

Marx, H., and G. B. Rabb. 1965. Relationships and zoogeography of the viperine snakes (Family Viperidae). Fieldiana: Zoology 44:161-206.

Matthews, R. K., and R. Z. Poore. 1981. Tertiary delta ${ }^{18} \mathrm{O}$ record and glacioeustatic sealevel fluctuations. Geology 8:501-504. 
Mayr, E. 1942. Systematics and the Origin of Species. Columbia University Press, New York.

Mayr, E. 1963. Animal Species and Evolution. Harvard University Press, Cambridge, Massachusetts.

McQuitty, L. L. 1966. Similarity analysis by reciprocal pairs for discrete and continuous data. Educational and Psychological Measurement 26:825-831.

Miles, D. B., R. Noecker, W. M. Roosenburg, and M. M. White. 2002. Genetic relationships among populations of Sceloporus undulatus fail to support present subspecific designations. Herpetologica 58:277-292.

Mitchell, J. C. 1977. Geographic variation of Elaphe guttata (Reptilia: Serpentes) in the Atlantic Coastal Plain. Copeia 1977:33-41.

Morrison, R. B. 1991. Introduction. Pp. 1-12. In R. B. Morrison (Ed.), Quaternary Nonglacial Geology: Conterminous U.S.: The Geology of North America, Volume K-2. The Geological Society of America, Boulder, Colorado.

Murphy, R. W., J. Fu, A. Lathrop, J. V. Feltham, and V. Kovac. 2002. Phylogeny of the rattlesnakes (Crotalus and Sistrurus) inferred from sequences of five mitochondrial DNA genes. Pp. 69-92. In G. W. Schuett, M. Hoggren, M. E. Douglas, and H. W. Greene (Eds.), Biology of the Vipers. Eagle Mountain Publishing, Eagle Mountain, Utah.

Murray, J. D. 1981. A pre-pattern formation mechanism for animal coat markings. Journal of Theoretical Biology 88:161-199.

Murray, J. D., and M. R. Myerscough. 1991. Pigmentation pattern formation on snakes. Journal of Theoretical Biology 149:339-360.

Nelson, G. J., and N. I. Platnick. 1981. Systematics and Biogeography: Cladistics and Vicariance. Columbia University Press, New York.

Nijhout, H. F. 1991. The Development and Evolution of Butterfly Wing Patterns. Smithsonian Institution Press, Washington D. C.

Nijhout, H. F. 2001. Elements of butterfly wing patterns. Journal of Experimental Zoology 291:213-225.

Nixon, K. C., and Q. D. Wheeler. 1990. An amplification of the phylogenetic species concept. Cladistics 6:211-223. 
Osgood, D. W. 1978. Effects of temperature on the development of meristic characters in Natrix fasciata. Copeia 1978:33-47.

Otte, D., and J. A. Endler. Speciation and its Consequences. Sinauer Associates, Sunderland, Massachusetts.

Parker, W. S., and M. V. Plummer. 1987. Population ecology. Pp. 253-301. In R. A. Seigel, J. T. Collins, and S. S. Novak (Eds.), Snakes: Ecology and Evolutionary Biology. Macmillan Publishing Company, New York.

Parkinson, C. L. 1999. Molecular systematics and biogeographical history of pitvipers as determined by mitochondrial ribosomal DNA sequences. Copeia 1999(3):576586.

Parkinson, C. L., K. R. Zamudio, and H. W. Greene. 2000. Phylogeography of the pitviper clade Agkistrodon: Historical ecology, species status, and conservation of cantils. Molecular Ecology 9:411-420.

Parkinson, C. L., J. A. Campbell, and P. T. Chippindale. 2002. Multigene phylogenetic analysis of pitvipers, with comments on their biogeography. Pp. 93-110. In G. W. Schuett, M. Hoggren, M. E. Douglas, and H. W. Greene (Eds.), Biology of the Vipers. Eagle Mountain Publishing, Eagle Mountain, Utah.

Peterson, C. R., A. R. Gibson, and M. E. Dorcas. 1993. Snake thermal ecology: The causes and consequences of body-temperature variation. Pp. 241-314. In R. A. Seigel and J. T. Collins (Eds.), Snakes: Ecology and Behavior. McGraw-Hill, New York.

Pisani, G. R., J. T. Collins, and S. R. Edwards. 1973. A re-evaluation of the subspecies of Crotalus horridus. Transactions of the Kansas Academy of Sciences 75:255263.

Pook, C. E., W. Wüster, and R. S. Thorpe. 2000. Historical biogeography of the western rattlesnake (Serpentes: Viperidae: Crotalus viridis), inferred from mitochondrial DNA sequence information. Molecular Phylogenetics and Evolution 15:269-282.

Potts, R., and A. K. Behrensmeyer. 1992. Late Cenozoic terrestrial ecosystems. Pp. 419-541. In Behrensmeyer, A. K., J. D. Damuth, W. A. DiMichele, R. Potts, H.D. Sues, and S. L. Wing (Eds.), Terrestrial Ecosystems through Time: Evolutionary Paleoecology of Terrestrial Plants and Animals. The University of Chicago Press, Chicago.

Pough, F. H., and J. D. Groves. 1983. Specializations of the body form and food habits of snakes. American Zoologist 23:443-454. 
Puorto, G., M. G. Salomão, R. D. G. Theakston, R. S. Thorpe, D. A. Warrell, and W. Wüster. 2001. Combining mitochondrial DNA sequences and morphological data to infer species boundaries: Phylogeography of lanceheaded pitvipers in the Brazilian Atlantic forest, and the status of Bothrops pradoi (Squamata: Serpentes: Viperidae). Journal of Evolutionary Biology 14:527-538.

Queral-Regil, A, and R. B. King. 1998. Evidence for phenotypic plasticity in snake body size and relative head dimensions in response to amount and size of prey. Copeia 1998:423-429.

Rage, J. C. 1987. Fossil history. Pp. 51-76. In R. A. Seigel, J. T. Collins, and S. S. Novak (Eds.), Snakes: Ecology and Evolutionary Biology. Macmillan Publishing Company, New York.

Reed, R. N. 2003. Interspecific patterns of species richness, geographic range size, and body size among New World venomous snakes. Ecography 26:107-117.

Reeder, T. W., and R. R. Montanucci. 2001. Phylogenetic analysis of the horned lizards (Phrynosomatidae: Phrynosoma): Evidence from mitochondrial DNA and morphology. Copeia 2001: 309-323.

Reichling, S. B. 1995. The taxonomic status of the Louisiana pine snake (Pituophis melanoleucas ruthveni) and its relevance to the evolutionary species concept. Journal of Herpetology 29:186-198.

Reinert, H. K., and R. T. Zappalorti. 1988. Timber rattlesnakes (Crotalus horridus) of the Pine Barrens: Their movement patterns and habitat preference. Copeia 1988:964-978.

Riser, J. A. M. 2001. Quaternary Geology and the Environment. Springer Verlag and Praxis Publishing, Berlin.

Rodríguez-Robles, J. A., and J. M. de Jesús-Escobar. 2000. Molecular systematics of new world gopher, bull, and pinesnakes (Pituophis: Colubridae), a transcontinental species complex. Molecular Phylogenetics and Ecology 14:3550 .

Rodríguez-Robles, J. A., D. F. Denardo, and R. E. Staubs. 1999. Phylogeography of the California mountain kingsnake, Lampropeltis zonata (Colubridae). Molecular Ecology 8:1923-1934.

Rosen, D. E. 1978. Vicariant patterns and historical explanation in biogeography. Systematic Zoology 27:159-188. 
Ross, H. H. 1965. Pleistocene events and insects. Pp. 583-596. In H. E. Wright, Jr., and D. G. Frey (Eds.), The Quaternary of the United States: A Review Volume for the VII Congress of the International Association for Quaternary Research. Princeton University Press, Princeton, New Jersey.

Rossman, C. E. 1980. Ontogenetic changes in skull proportions of the diamondback water snake, Nerodia rhombifera. Herpetologica 36:42-46.

Rossman, D. A. 1962. Thamnophis proximus (Say), a valid species of garter snake. Copeia 1962:741-748.

Rossman, D. A. 1963. The colubrid snake genus Thamnophis: A revision of the sauritus group. Bulletin of the Florida State Museum, Biological Sciences 7:99-178.

Rossman, D. A., N. B. Ford, and R. A. Seigel. 1996. The Garter Snakes: Evolution and Ecology. University of Oklahoma Press, Norman, Oklahoma.

SAS Institute. 1989. SAS/STAT User's Guide, Volumes 1 and 2, version 6, 4th edition. SAS Institute, Cary, North Carolina.

SAS Institute. 2000. Base SAS software for personal computers, version 8.01. SAS Institute, Cary, North Carolina.

Savitzky, A. H. 1980. The role of venom delivery strategies in snake evolution. Evolution 34:1194-1204.

Schneider, G. E. 1986. Geographic variation in the contact zone of two subspecies of the Pacific rattlesnake, Crotalus viridis oreganus and Crotalus viridis helleri. M.A. Thesis, University of California, Santa Barbara, California.

Sharma, S. 1996. Applied Multivariate Techniques. John Wiley and Sons, New York.

Shine, R. 1993. Sexual dimorphism in snakes. Pp. 49-86. In R. A. Seigel and J. T. Collins (Eds.), Snakes: Ecology and Behavior. McGraw-Hill, New York.

Shine, R. 1994. Sexual size dimorphism in snakes revisited. Copeia 1994:326-346.

Shine, R. 2002. Do dietary habits predict scale counts in snakes? Journal of Herpetology 36:268-272.

Simpson, G. G. 1961. Principles of Animal Taxonomy. Columbia University Press, New York.

Sites, J. W., Jr., and J. R. Dixon, 1982. Geographic variation in Sceloporus variabilis, and its relationship to $S$. teapensis (Sauria: Iguanidae). Copeia 1982:14-27. 
Smith, H. M. 1990. The universal species concept. Herpetologica 46:122-124.

Sneath, P. H. A., and R. R. Sokal. 1973. Numerical Taxonomy: The Principles and Practice of Numerical Classification. W. H. Freeman and Company, San Francisco, California.

Sokal, R. R., and C. D. Michener. 1958. A statistical method for evaluating systematic relationships. University of Kansas Science Bulletin 38:1409-1438.

Sorenson, T. 1948. A method of establishing groups of equal amplitude in plant sociology based on similarity of species content and its application to analyses of the vegetation on Danish Commons. Biologiske Skrifter 5:1-34.

Thomas, R. A. 1974. Geographic variation in Elaphe guttata (Linnaeus) (Serpentes: Colubridae). M.S. Thesis, Texas A \& M University, College Station, Texas.

Thorpe, R. S., R. P. Brown, A. Malhotra, and W. Wüster. 1991. Geographic variation and population systematics: Distinguishing between ecogenetics and phylogenetics. Bolletini di Zoologia 58:329-335.

Thorpe, R. S., A. Malhotra, H. Black, J. C. Daltry, and W. Wüster. 1995. Relating geographic pattern to phylogenetic process. Philosophical Transactions of the Royal Society of London B 349:61-68.

Thorpe, R. S., R. P. Brown, M. Day, A. Malhotra, D. P. McGregor, and W. Wüster. 1994. Testing ecological and phylogenetic hypotheses in microevolutionary studies. Pp. 189-206. In P. Eggleton and R. Vane-Wright (Eds.), Phylogenetics and Ecology. Academic Press, London.

Turing, A. M. 1952. The chemical basis of morphogenesis. Philosophical Transactions of the Royal Society of London 237B:37-72.

United States Fish and Wildlife Service. 1999. Endangered and Threatened Wildlife and Plants. 50 CFR 17.11 and 17.12. Department of the Interior, Washington, D. C.

Van Devender, T. R., and R. Conant. 1990. Pleistocene forests and copperheads in the eastern United States, and the historical biogeography of New World Agkistrodon. Pp. 601-614. In H. K. Gloyd and R. Conant, Snakes of the Agkistrodon Complex: A Monographic Review. Society for the Study of Amphibians and Reptiles, Contributions to Herpetology 6:1-614.

Wiens, J. J. 1999. Polymorphism in systematics and comparative biology. Annual Review of Ecology and Systematics 30:327-362. 
Wiens, J. J., and T. W. Reeder. 1997. Phylogeny of the spiny lizards (Sceloporus) based on molecular and morphological evidence. Herpetological Monographs 11:1-101.

Wiley, E. O. 1978. The evolutionary species concept reconsidered. Systematic Zoology 27:17-26.

Wiley, E. O. 1981. Phylogenetics: The Theory and Practice of Phylogenetic Systematics. John Wiley and Sons, New York.

Williams, K. L. 1978. Systematics and natural history of the American milk snake, Lampropeltis triangulum. Milwaukee Public Museum Publications in Biology and Geology 2:1-258.

Wilson, E. O., and W. H. Brown. 1953. The subspecies concept and its taxonomic applications. Systematic Zoology 2:97-111.

Wüster, W., and R. S. Thorpe. 1992. Asiatic cobras: Population systematics of the Naja naja species complex (Serpentes: Elapidae) in India and Central Asia. Herpetologica 48:69-85.

Wüster, W., S. Otsuka, A. Malhotra, and R. S. Thorpe. 1992. Population systematics of Russell's viper: A multivariate study. Biological Journal of the Linnean Society 47:97-113.

Wüster, W., R. S. Thorpe, G. Puorto, and BBBSP. 1996. Systematics of the Bothrops atrox complex (Reptilia: Serpentes: Viperidae) in Brazil: A multivariate analysis. Herpetologica 52:263-271.

Wüster, W., M. G. Salomão, G. J. Duckett, R. S. Thorpe, and BBBSP. 1999. Mitochondrial DNA phylogeny of the Bothrops atrox species complex (Squamata: Serpentes: Viperidae). Kaupia 8:135-144.

Wüster, W., M. G. Salomão, J. A. Quijada-Mascareñas, R. S. Thorpe, and BBBSP. 2002. Origins and evolution of the South American pitviper fauna: Evidence from mitochondrial DNA sequence analysis. Pp. 111-128. In G. W. Schuett, M. Hoggren, M. E. Douglas, and H. W. Greene (Eds.), Biology of the Vipers. Eagle Mountain Publishing, Eagle Mountain, Utah.

Zamudio, K. R., and H. W. Greene. 1997. Phylogeography of the bushmaster (Lachesis muta: Viperidae): Implications for neotropical biogeography, systematics, and conservation. Biological Journal of the Linnean Society 62:421-442.

Zamudio, K. R., K. B. Jones, and R. H. Ward. 1997. Molecular systematics of shorthorned lizards: Biogeography and taxonomy of a widespread species complex. Systematic Biology 46:284-305. 


\section{APPENDIX I}

\section{SPECIMENS EXAMINED}

Museum acronyms follow Leviton et al. (1985). Each specimen is identified as male (m), female (f), or sex undetermined (u).

Collection Acronyms.-AMNH: American Museum of Natural History, New York. ANSP: Academy of Natural Sciences, Philadelphia. APSU: Austin Peay State University, Clarksville. ARK: University of Arkansas Museum, Fayetteville. AUM: Auburn University, Auburn. BEECS: Biotechnologies for the Ecological, Evolutionary, and Conservation Sciences, Genetic Analysis Lab, Gainesville. BMNH: Bell Museum of Natural History, St. Paul. CA: Chicago Academy of Sciences, Chicago. CM: Carnegie Museum of Natural History, Pittsburgh. CU: Cornell University Museum of Vertebrates, Ithaca. FMNH: Field Museum of Natural History, Chicago. DNHS: Illinois Natural History Survey, Champaign. KU: University of Kansas Natural History Museum, Lawrence. LSUMZ: Louisiana State University Museum of Natural Science, Baton Rouge. MCZ: Museum of Comparative Zoology, Cambridge. MPM: Milwaukee Public Museum, Milwaukee. MUSU: Murray State University, Murray. NCSM: North Carolina Museum of Natural Sciences, Raleigh. NLU: University of Louisiana Museum of Natural History, Monroe. NWU: Northwestern University, Evanston (in CA). NYSM: New York State Museum, Albany. ODU: Old Dominion University, Norfolk. OMNH: Oklahoma Museum of Natural History, Norman. OUVC: Ohio University Vertebrate Collection, Athens. SDSNH: San Diego Natural History Museum, San Diego. SIUC: Southern Illinois University Fluid Vertebrate Collections, Carbondale. TCWC: Texas Cooperative Wildlife Collection, College Station. TMC: Thomas More College, Crestview Hills. TU: Tulane University Museum of Natural History, New Orleans. UF: Florida State Museum, Gainesville. UGAMNH: University of Georgia Museum of Natural History, Athens. UIMNH: University of Illinois Museum of Natural History, Champaign. UL: University of Louisville, Louisville. UMMZ: University of Michigan Museum of Zoology, Ann Arbor. UNSM: University of Nebraska State Museum, Lincoln. USNM: National Museum of Natural History, Washington. UTA: University of Texas, Arlington. UTEP: University of Texas, El Paso. WVBS: West Virginia Biological Survey, Marshall University, Huntington.

Alabama.-Baldwin: AUM 10375 (m). Barbour: AUM 4807 (m), 4970 (m), 5997 (m), 7275 (f), 21128 (m), 21441 (m); UF 19278 (f), 19279 (f). Blount: CM 60276 (m).

Bullock: UF 47914 (m); USNM 520883 (f). Butler: AUM 4290 (m), 9483 (m), 9490 (m); CM 69451 (m); UF 66821 (f); UMMZ 130216 (f). Chambers: AUM 1820 (m), 21178 (m). Clay: UF 72646 (m). Cleburne: AUM $417(\mathrm{~m}), 418$ (m), 3019 (f), 5095 (f). Coffee: AUM 9094 (f), 20704 (m). Conecuh: AUM 4808 (m), 19179 (f). Dallas: AUM 396 (m), 18553 (m); LSUMZ 22862 (f); TU 21345 (f). Dekalb FMNH 95229 (m), 95230 (m), 95231 (m); INHS 8411 (m); KU 84507 (f); USNM 307982 (m). Elmore: AUM 21162 (f). Hale: UIMNH 53019 (f). Jackson: AUM 9698 (m). Lawrence: AUM 5626 (f). Lee: AUM $419(\mathrm{~m}), 1849(\mathrm{~m}), 1859(\mathrm{~m}), 2864(\mathrm{~m}), 2865(\mathrm{~m}), 4589$ (f), 10840 (f), $13670(\mathrm{~m}), 15787$ 
(m), 18313 (m), 32661 (m), 32824 (m), 32836 (m); CM 9897 (f); UF 74514 (f). Macon: AUM 5751 (f), 5996 (m); AUM 16946 (f). Monroe: TU 18314 (f). Montgomery: AUM 3017 (m); USNM 307983 (m). Perry: AUM 10723 (f); CM 72139 (m). Pike: AUM 4546 (m), 21607 (f); UMMZ 125657 (m), 125701 (6m:1f). Russell: AUM 5570 (f), 11271 (f), 21573 (m); USNM 313387 (m). Shelby: AUM 3020 (m). Sumter: AUM 16898 (m); CM 9915 (f), 9916 (f). Tallapoosa: AUM 10802 (m), 28505 (f). Tuscaloosa: AUM 3018 (f); CA 6162 (f). Walker: CM 71989 (f). Washington: AUM 23375 (f). Wilcox: TU 28846 (m); UMMZ 67756 (f). Winston: AUM 18443 (m); LSUMZ 30909 (m). No County: CM $33860(\mathrm{~m})$; UF $116074(\mathrm{~m})$.

Arkansas.-Arkansas: UF 47950 (f). Cleveland: MPM 18628 (m). Columbia: MPM 15166 (f). Cross: UTA 14697 (f), 14698 (f). Dallas: LSUMZ 43773 (m). Franklin: ARK 852 (f). Hot Spring: FMNH 91 (m). Lafayette: MPM 7563 (f). Lawrence: UMMZ 79422 (m). Lincoln: ARK 880 (m). Logan: ANSP 15564 (m). Phillips: CM 25058 (m); LSUMZ 20280 (m). Polk: FMNH 28439 (f), 29420 (f); UTA 26532 (m), 33820 (m). Pulaski: MPM 18636 (f). St. Francis: CA 7031 (f), 7032 (m), 7034 (m). Stone: TU 28848 (m); UMMZ 155652 (m), 155653 (m). Washington: ARK 872 (m), 873 (f). White: MPM 14451 (f), 14452 (f), $15683(\mathrm{~m})$.

Connecticut-Hartford: AMNH 127461 (u), 130649 (m), 130650 (m), 134297 (m), 136759 (m), 136760 (m), 137722 (m), 137723 (u), 139111 (m), 139112 (m); UMMZ 152985 (m); USNM 139271 (f). Litchfield: AMNH 130117 (f), 135238 (f), 136761 (m), 142235 (f); UMMZ 99716 (m). Middlesex: AMNH 136762 (f); USNM 139226 (m), 139227 (m). No County: FMNH 15683 (m).

Florida-Alachua: UF 14575 (f), 14576 (f), 42571 (m), 44270 (m), 44960 (m), 65267 $(\mathrm{m}), 66996(\mathrm{~m}), 66997(\mathrm{~m}), 66999(\mathrm{~m}), 67000(\mathrm{~m}), 67001(\mathrm{~m}), 67002(\mathrm{~m}), 67003(\mathrm{~m})$, $67004(\mathrm{~m}), 67005(\mathrm{~m}), 67006(\mathrm{~m}), 67007(\mathrm{f}), 67008(\mathrm{f}), 71482(\mathrm{f}), 81527(\mathrm{~m}), 81712(\mathrm{~m})$, 87970 (f), 109824 (f), 109825 (f); UMMZ 175526 (f). Baker: BEECS 384 (m); UF 42564 (f), 42566 (f), 42567 (f), 67009 (f), 67010 (m), 67011 (m); UMMZ 175855 (f); USNM 210092 (m). Bradford: UF 18349 (m), 47913 (m), 67012 (f), 67013 (f), 67014 (f), 67015 (m), 67016 (m). Columbia: BEECS 385 (f); CA 5854 (m), 5855 (m); UF 1385 (m), 9650 (f), 24691 (f), 42568 (f), 67017 (m), 67018 (m), 67019 (f), 67020 (f), 67022 (m), 91659 (m); USNM 210093 (f). Duval: UF 72641 (f). Hamilton: UF 25046 (f). Nassau: UF $42563(\mathrm{~m})$; UTEP $14203(\mathrm{~m})$. Suwannee: AMNH $4078(\mathrm{u}), 4079(\mathrm{u})$; CA 15243 (f); UF $16162(\mathrm{~m}), 19732$ (f), $19733(\mathrm{~m}), 19734(\mathrm{~m})$. Union: UF $1184(\mathrm{f}), 6615$ (f), $67023(\mathrm{~m})$, 85225 (m). Walton: UF 91594 (f). No County: KU 61308 (m); UF 13156 (m), 74694 (m), $92010(\mathrm{~m})$.

Georgia._Baker: CM 40031 (m), 40186 (f), 40187 (f), 40188 (f), 40189 (f), 40190 (f), 40192 (f); UF 66820 (m), 116089 (f), 116090 (f). Banks: UGAMNH 20858 (m), 20859 (m). Bleckley: UF 116084 (f), 116085 (f), 116086 (m). Brooks: UF 116088 (m). Burke: UGAMNH 4799 (m). Calhoun: UGAMNH $4783(\mathrm{~m})$. Camden: BEECS $266(\mathrm{~m})$; CM 56368 (f); UGAMNH 19105 (f). Charlton: CA 4119 (m); UF 116095 (f); UGAMNH 19462 (m). Chatham: BEECS 264 (m); CM 27759 (m). Chattahoochee: USNM 520887 
(m). Cherokee: UGAMNH 4773 (m). Clarke: UGAMNH 4775 (f), 20781 (f). Clay: UF 116094 (m). Clinch: KU 177058 (f); UGAMNH 4781 (m), 4791 (f). Cobb: LSUMZ 38644 (f). Colquitt: UGAMNH 19080 (m). Dougherty: UGAMNH 4794 (f). Echols: UGAMNH 4790 (f). Fannin: UGAMNH 4767 (m). Glynn: BEECS 265 (f); UGAMNH 4787 (f); USNM 166374 (f). Grady: UF 21631 (f). Habersham: UF 116093 (m). Hancock: UGAMNH 4785 (f). Harris: USNM 520856 (m), 520857 (f), 520858 (m), 520859 (f), $520861(\mathrm{~m}), 520862(\mathrm{f}), 520863(\mathrm{~m}), 520864(\mathrm{f}), 520865(\mathrm{~m}), 520866(\mathrm{~m}), 520867(\mathrm{~m})$, $520868(\mathrm{~m}), 520870(\mathrm{~m}), 520872(\mathrm{f}), 520873(\mathrm{~m}), 520874(\mathrm{~m}), 520875(\mathrm{~m}), 520876(\mathrm{~m})$, $520878(\mathrm{~m}), 520884(\mathrm{~m}), 520885(\mathrm{~m}), 520886(\mathrm{f})$. Houston: UGAMNH $4795(\mathrm{~m})$; UMMZ 100662 (f). Jefferson: CA 15889 (m), 15890 (f), 15891 (m), 15892 (m), 16216 (f), 16217 (f), 16218 (m); UGAMNH 4793 (f). Johnson: UF 101059 (f). Liberty: AMNH 112931 (f); TU 18577 (f). Lowndes: UF 72642 (f). Macon: UGAMNH 27343 (m). Marion: USNM $520871(\mathrm{~m})$. McDuffie: CA 15362 (m). McIntosh: UGAMNH 19176 (f), 37089 (m), 40075 (f). Meriwether: USNM 520855 (m). Monroe: UGAMNH 27344 (m). Morgan: UGAMNH 4771 (f). Muscogee: USNM 520860 (m), 520877 (f). Pickens: CA 17135 (m). Quitman: UF 87163 (f), 116083 (f). Rabun: UF 116091 (f); UGAMNH 4776 (f), 4777 (m), 4780 (m). Richmond: AMNH 99095 (m); BEECS 417 (f); UF 116082 (m). Stephens: UGAMNH 4768 (m), 4774 (m). Stewart: USNM 520869 (f). Talbot: USNM 520881 (m). Terrell: UGAMNH 20699 (m). Treutlen: UMMZ 155650 (f). Twiggs: UGAMNH 4772 (f). Union: UGAMNH 4800 (f). Upson: CM 67405 (f). Ware: UGAMNH 4770 (f), 4782 (m), 19081 (f), 19431 (m), 19432 (f). Warren: UF 116087 (m), 116092 (m). Washington: UF 19128 (m); KU 68960 (f). No County: AMNH 50496 (m); UF 68585 (m), 68586 (f), 72643 (f), 84096 (m); ODU 484 (f), 485 (f), 486 (f), 487 (f), 489 (f), 491 (f). UGAMNH 40124 (f), 40125 (m).

Illinois.-Adams: FMNH 38242 (f), 38243 (m); SDSNH 9324 (m), 9325 (f). Alexander: INHS 2260 (f). Effingham: INHS $8169(\mathrm{~m})$. Greene: INHS $1548(\mathrm{~m})$. Hancock: MPM 671 (f). Jackson: CA 4462 (f), 4463 (m), 6171 (f), 6172 (m), 12483 (m); FMNH 18657 (f), 18658 (f), 162944 (f), 191052 (f), 204659 (m); INHS 1814 (f), 1815 (f), 1922 (f), 2555 (f), 5360 (m); OMNH 26862 (f), 26863 (f); SDSNH 4387 (f); SIUC 90 (f), 197 (f), 306 (m), 1107 (f), 1285 (f), 1629 (m), 1630 (m), 1631 (m), 2070 (f), 2884 (f), 2885 (m), 3747 (m), 3750 (m); UF 14577 (m), 16018 (m); UIMNH 1214 (f), 1215 (f), 1216 (f), 84782 (f); UMMZ 68477 (f), 79400 (m). Jasper: INHS 1551 (m). Jersey: FMNH 19201 (f); INHS 1654 (m), 11251 (f), 12010 (f), 12852 (f). Jo Daviess: CA 3979 (m), 3980 (m), 3981 (m), 3982 (f), 4461 (f), 7480 (m); INHS 1552 (f), 1553 (m), 1554 (f), 1555 (f), 12086 (f); SIUC 2887 (f); UMMZ 79429 (1m:1f). Johnson: SIUC 111 (m). LaSalle: INHS 9646 (f). Madison: SIUC 2684 (m). Monroe: INHS 4334 (f), 4335 (f), 4336 (m); SIUC 3130 (f). Perry: INHS 1549 (f); UF 74513 (f). Pike: INHS 3661 (f), 3662 (f), 5832 (m); UIMNH 41320 (f), 50961 (f), 52103 (f). Pope: SIUC 2220 (m). Rock Island: INHS 3704 (m). Union: FMNH 23736 (f), 203996 (f), 203997 (m), 203998 (m), 204002 (m), 204081 (f); INHS 1406 (f), 1407 (f), 1550 (m), 1556 (m), 2525 (m), 8790 (f); SIUC 205 (m), 979 (f), 1534 (m), 1931 (f), 1971 (f), 2035 (m), 2069 (f), 2490 (m), 2499 (f).

Indiana.--Brown: CA 14225 (m), 14226 (f), 14227 (f), 14228 (f), 14229 (f), 14230 (f), 14231 (m), 14232 (m), 14233 (m), $14234(\mathrm{~m}), 14235(\mathrm{~m}), 14236$ (f), $14237(\mathrm{~m}), 14238$ 
(m), 14239 (m), 15053 (m), 15054 (f), 15647 (m), 15648 (f), 15649 (m), 15650 (f); UMMZ 99061 (f). Jackson: UMMZ 105573 (f), 110383 (m). Lawrence: UMMZ 110384 (m); Martin: UMMZ 108080 (m); Monroe: UMMZ 101846 (f), 106666 (f). Morgan: UMMZ 106667 (m).

Iowa. -Clinton: UNSM 1130 (m). Henry: FMNH 41045 (m), 41046 (f). Jackson: UIMNH 50962 (f). Jones: CA 4458 (f), 4459 (f), 4460 (f), 13680 (f), 17804 (f), 17805 (f), 17806 (m), 17807 (f), 17808 (m), 18321 (f), 18322 (f), 18323 (f). Lee: SDSNH 36069 (f), 36070 (f), 36071 (m). Madison: SDSNH 36068 (m), 36072 (f), 36073 (f), 36074 (f), 36075 (m), 36076 (m), 36077 (f), 36078 (f), 36079 (f), 36080 (m), 36081 (f), 36082 (f), 36083 (m), 36084 (m), 36085 (f), 36086 (f), 36087 (f), 36088 (f), 36089 (f), 36090 (m), 36091 (f); UMMZ 92868 (f), 92869 (m). No County: MPM 1860 (m).

Kansas.-Anderson: KU 192415 (m); Atchison: AMNH 64124 (m); CA 9429 (f), 9430 (f); KU 189063 (f); UF 116096 (m), 116097 (f), 116098 (m), 116099 (f). Bourbon: KU 31468 (f); UMMZ 68484 (m), 79402 (m). Chautauqua: KU 159805 (m), 192441 (m), 197089 (f). Crawford: KU 187736 (f); OMNH 38052 (f). Doniphan: USNM 197651 (m). Douglas: CM 58561 (m); FMNH 8485 (f), 124405 (u); KU 1640 (m), 1641 (m), 1644 (f), 1645 (f), 1646 (f), 1649 (m), 1650 (m), 1651 (f), 1652 (m), $1653(\mathrm{~m}), 1654$ (f), $1655(\mathrm{~m})$, $1656(\mathrm{~m}), 1659$ (f), 1812 (f), 2319 (m), 2320 (f), $2321(\mathrm{~m}), 2321(\mathrm{~m}), 28734$ (f), 154056 (f), $154057(\mathrm{~m}), 154058$ (f), 154059 (f), 154060 (f), $154061(\mathrm{~m}), 154062$ (f), $154063(\mathrm{~m})$, 186742 (f), 207324 (m), 224651 (f), 288641 (m); OMNH 26964 (f), 26973 (m); TCWC 3278 (f); UIMNH 1217 (f), 19175 (f), 19176 (f), 19177 (f); UTA 10990 (f), 10991 (f), 10992 (f). Elk: KU 206505 (m). Franklin: CA 1352 (f), 1353 (f), 1354 (f), 1355 (f), 1356 (f), $1357(\mathrm{~m}), 5636(\mathrm{~m}), 5637$ (f), $5638(\mathrm{~m}), 5639(\mathrm{~m}), 5640$ (f), 5641 (m), 5642 (f), 5643 (m), 5644 (m), 5645 (f), 5646 (m), 5647 (f), 5648 (f); CM 8650 (f); KU 55243 (m), $55244(\mathrm{~m}), 55245(\mathrm{~m}), 55246(\mathrm{~m}), 55247(\mathrm{~m}), 55248(\mathrm{~m}), 55249(\mathrm{f}), 55250(\mathrm{~m}), 55251$ (f), 55252 (f), 177059 (f), 177061 (f), 177062 (f), 177063 (f), 203842 (f); UMMZ 68485 (m), $68486(\mathrm{f}), 68487(\mathrm{~m}), 68488(\mathrm{f}), 68489(\mathrm{f}), 68490(\mathrm{~m}), 68491(\mathrm{f}), 68492(\mathrm{~m}), 68493$ (m), 68494 (m), 79399 (2m:3f), 79401 (1m:2f). Geary: CM 9145 (f). Jackson: KU 72854 (m). Jefferson: FMNH 191388 (m); TU 18565 (f); UIMNH 18260 (f). Johnson: KU 84502 (f), 84505 (f), 84506 (f), 186743 (m), 189066 (m), 220065 (m). Leavenworth: KU 1661 (f), 1662 (f), 1663 (m), 1664 (m), 1667 (f), 30071 (m), 204739 (m), 211598 (f). Linn: KU 84503 (f); UMMZ 96882 (m), 79412 (2m). Marshall: CA 1572 (f); UMMZ 68478 (f), 68479 (f), 68480 (f), 68481 (f), 68482 (f), 79404 (1m:2f), 79406 (2m), 79430 (2m), 79431 (2f). Miami: CA 4455 (m), 4456 (f), 4457 (f); KU 22012 (f), 84504 (m), 182337 (f); UIMNH 21199 (f); UMMZ 68467 (m), 68468 (f), 68469 (f), 68470 (m), $68471(\mathrm{f}), 68472(\mathrm{~m}), 68473(\mathrm{f}), 68474(\mathrm{f}), 68475(\mathrm{~m}), 68476(\mathrm{~m}), 79403(1 \mathrm{~m}: 2 \mathrm{f}), 79405$ (1m:1f), 79407 (2f), 79408 (2m), 79409 (m), 79410 (1m:5f), 79411 (f); UTEP 5103 (f), 5104 (f), 5105 (m), 5122 (f). Montgomery: KU 221491 (m); Osage: KU 19220 (f), 19282 (f), 30057 (f). Riley: KU 192704 (m). Riley/Geary: ANSP 7123 (m). Shawnee: KU 159804 (m); UIMNH 1653 (f). Wabaunsee: KU 223469 (m). Wilson: KU 216205 (m). Wyandotte: KU 2362 (f). No County: UF 73256 (f). 
Kentucky.-Breathitt: TMC 924 (m), 925 (m). Edmonson: KU 19196 (f). Jefferson: UF $53397(\mathrm{~m})$; UL 3735 (m). Laurel: UMMZ $135301(\mathrm{~m})$. Lyon: APSU $249(\mathrm{~m}), 406(\mathrm{~m})$, 421 (f), 427 (m), 449 (f), 924 (m), 937 (m), 3716 (m), 5061 (f); KU 137861 (m). Nelson: UIMNH 41084 (m). Rowan: KU 144656 (m). Trigg: APSU $5518(\mathrm{~m})$; CA 6944 (m); CM 41524 (f); KU 144655 (m); MUSU 1512 (m). Wayne: UL 2395 (m), 2396 (m). No County: CM 25795 (m); UL 4457 (m).

Louisiana.-Beauregard: TU 28888 (m). Bienville: USNM 291905 (m). Catahoula: NLU 47062 (m). Concordia: NLU 10370 (m). De Soto: NLU 100002 (f), 100003 (m). East Baton Rouge: LSUMZ 18540 (m), 20203 (m), 20222 (m), 20223 (f), 24239 (m), 36980 (m); MPM 18396 (m). East Feliciana: LSUMZ 58335 (f). Evangeline: LSUMZ 30917 (m). Franklin: NLU 9090 (f). Iberville: KU 145980 (f); LSUMZ 24351 (m), 46839 (f). Jefferson: LSUMZ 55288 (m); TU 18034 (m). Livingston: LSUMZ 57802 (m), 80835 (m), 80836 (f). Morehouse: NLU 70479 (m), 100005 (m); USNM 322064 (m).

Natchitoches: CM 69450 (m); LSUMZ 49582 (f), 58462 (f). Orleans: FMNH 5561 (m); TU 6083 (f), 11716 (f), $14226(\mathrm{~m}), 17700$ (f), $18220(\mathrm{~m}), 28887(\mathrm{~m})$. Ouachita: KU 289568 (m), 289615 (m); NLU 5764 (f), 9582 (m), 19924 (m), 20800 (f), $36269(\mathrm{~m})$, 46978 (f), 70137 (m), 70206 (f), 70485 (m). Plaquemines: TU 5637 (f), 10993 (f), 18578 (m), 21299 (f), $21346(\mathrm{~m}), 21924(\mathrm{~m}), 21925(\mathrm{f}), 21938(\mathrm{~m}), 23758(\mathrm{~m}), 25751(\mathrm{~m})$, 28509 (m), 28514 (m), 28905 (m); USNM 238631 (f), 238632 (f), 238633 (f), 238634 (m), 238635 (f); Point Coopee: LSUMZ 16696 (m); TU 5502 (m). Rapides: LSUMZ 58475 (f); Sabine: LSUMZ 20178 (f); St. Bernard: LSUMZ 80795 (m); St. Charles: TU 18303 (m), 18359 (m). St. Helena: LSUMZ 17991 (m); St. John The Baptist: UTEP 15132 (m); St. Landry: LSUMZ 20204 (f), 31535 (m). St. Mary: LSUMZ 74836 (m). Terrebonne: TCWC 71395 (f), 71445 (f). Union: TU 12745 (f). Vernon: UTEP 15131 (m). Washington: LSUMZ 80839 (m). West Baton Rouge: LSUMZ 9132 (m), 30328 (f), 56185 (f), 56265 (m). West Feliciana: LSUMZ 37584 (m), 80838 (m).

Maryland.-Frederick: CA 7387 (f); INHS 4393 (f), 4450 (m), 4451 (f), 4452 (m); UF 41136 (m); UIMNH 2947 (m), 2949 (f), 2950 (f), 2951 (f), 2952 (m), 2953 (m), 2954 (f), 2955 (f), 2956 (m), 2957 (f), 2958 (f), 2959 (f), 2960 (m), 2961 (m), 2962 (m), 2963 (f), 2964 (m); USNM 268751 (f). Garrett: CA 7383 (f); CM 1326 (m), 13681 (f), 13875 (m), 30437 (f).

Massachusetts.-Berkshire: AMNH 23088 (m), 66592 (m), 66593 (m), 66594 (f); MCZ 27543 (m), 27544 (f), 45801 (f), 82302 (m), 82303 (m); NYSM 2628 (f), 2629 (f); UMMZ 121023 (f). Essex: SDSNH 30403 (f), 30404 (f); SDSNH 30405 (f). Franklin: MCZ 60863 (m), 60864 (m), 143939 (f), 147402 (m). Norfolk: AMNH 44949 (m); MCZ 19191 (f), 19192 (m), 49107 (f), 153966 (m), 162019 (f), 176370 (m); SDSNH 30406 (m). Worchester: UF 4915 (f). No County: AMNH 38221 (f), 38224 (m).

Minnesota.-Fillmore: BMNH 225 (m), 232 (1m:1f), 233 (f), 235 (f), 236 (m), 1213 (f). Goodhue: BMNH 895 (f), 896 (f). Olmsted: BMNH 1414 (f), 1415 (f), 1444 (f), 1445 (f). Wabasha: BMNH 97 (f), 144 (f); USNM 313388 (m). Washington: BMNH 2671 (m). Winona: BMNH 1361 (f), 1446 (f), 2006 (f); FMNH 39466 (m). No County: BMNH 158 
(m), 1344 (f), 1345 (m), 1346 (f), 1347 (f), 1348 (f), 1349 (f), 1350 (m), 1351 (m), 1352 (f), 1358 (f), 1359 (f), 1360 (m), 13563 (m); NWU 587 (f), 588 (f), 589 (f), 590 (m), 591 (m), $593(\mathrm{~m}), 594(\mathrm{f})$.

Mississippi.-Amite: LSUMZ 33533 (m). Benton: ANSP 7107 (m). Bolivar: CA 5795 (m), 5851 (m), $6981(\mathrm{~m})$; FMNH 3502 (m). Holmes: UTA 534 (m). Kemper: KU 204348 (m). Lafayette: UTEP 16594 (f). Lauderdale: LSUMZ 38646 (f). Lowndes: UMMZ 156000 (f). Marion: TU 28904 (m). Noxubee: FMNH 194591 (f). Oktibbeha: UMMZ 152106 (f), 152111 (m). Panola: UTEP 16596 (m), 16597 (f), 16598 (f). Wilkinson: LSUMZ $56250(\mathrm{~m}), 56277(\mathrm{~m})$.

Missouri-Andrew: KU 220064 (f). Barton: KU 2322 (m). Bates: KU 84474 (m), 84475 (m). Boone: CA 8388 (f), 8389 (m), 8600 (m); UNSM 625 (f). Caldwell: KU 84440 (f), 84441 (f), 84471 (f), 84473 (m). Carroll: KU 84463 (m). Carter: UMMZ 70742 (f). Cass: KU 84466 (m). Chariton: CA 8765 (m). Clay: CA 8694 (f); KU 84436 (m), 84465 (m). Dunklin: USNM 56739 (f). Holt: KU 84446 (m), 84462 (f), 84476 (f), 177064 (f), 177069 (m). Jackson: CA 8598 (m), 10727 (m), 10728 (f), $10729(\mathrm{~m}), 10730(\mathrm{~m}), 10731$ (f), 10732 (f), 10733 (f), 10734 (f), 10735 (f), 10736 (f), 10737 (f), 10738 (m), 10739 (m), 10740 (m), 10741 (f), 10742 (f); CM 9143 (f), 9144 (f); KU 84422 (m), 84423 (m), 84426 (m), 84427 (m), 84429 (f), 84430 (m), 84434 (f), 84439 (f), 84447 (m), 84467 (m), 84469 (f), 84472 (m), 84479 (f), 84480 (m), 84481 (f), $84484(\mathrm{~m}), 84492(\mathrm{~m}), 84493(\mathrm{~m})$, 84494 (f), 84496 (f), 84499 (m), 84692 (m), 177065 (f), 177066 (f), 177067 (m); MPM 13849 (f); OMNH 26539 (m), 26540 (m), 26541 (m); TCWC 18382 (m); UMMZ 68495 (f). Johnson: KU 84424 (f), 84431 (m), 84442 (f), 84477 (m); UTA 1327 (m). Lafayette: KU 84428 (f). Livingston: CA 8744 (m). Osage: KU 289658 (m). Pettis: CA $6174(\mathrm{~m})$, 8594 (m); KU 177068 (m); MPM 9044 (m), 13842 (f). Pike: KU 84433 (m), 84435 (m), 84485 (f), 84486 (f), 84487 (m), 84488 (m), 84489 (m), 84490 (m). Platte: KU 84425 (f), 84491 (m). Ray: KU 84432 (m), 84437 (m), 84438 (m), 84443 (m), 84444 (f), 84445 (f), 84448 (m), 84449 (m), 84450 (f), 84451 (f), 84452 (f), 84453 (f), 84454 (f), 84455 (f), 84456 (f), 84457 (f), 84458 (m), 84459 (m), 84460 (m), 84461 (f), 84464 (f), 84468 (f), 84470 (f), 84478 (f), 84483 (f), 84495 (f), 84497 (f), 84498 (f), 84500 (f). Saline: KU 84482 (m). Scott: SDSNH 23861 (m), 23862 (f), 23863 (f). St. Genevieve: USNM 56738 (m). St. Louis: CM 6370 (f), $6371(\mathrm{~m})$; UF $13116(\mathrm{~m}), 13117$ (m), 13118 (f). Stoddard: CA $5029(\mathrm{~m}), 8595(\mathrm{~m}), 8596(\mathrm{~m}), 8597(\mathrm{~m}), 8599(\mathrm{~m}), 8601(\mathrm{~m}), 8602(\mathrm{f}), 8603$ (f), 8604 (f). Stone: ANSP 7108 (f); SDSNH 3275 (f). Warren: KU 289659 (m). Wayne: CA 8774 (m); KU 84508 (m). No County: NWU 175 (f).

Nebraska.-Cass: UNSM 2030 (m). Gage: UNSM 620 (f), $621(\mathrm{~m}), 622(\mathrm{~m}), 647$ (f), 1295 (f), 1296 (f), 1389 (f), 2031 (f), 6905 (m), 7013 (f), 8339 (f), 8757 (f). Jefferson: UNSM 15110 (m). Richardson: UNSM 2028 (f), 2029 (f).

New Jersey.-Burlington: AMNH 46392 (m), 50995 (f), 57774 (m), 63833 (f), 63834 (m), 63835 (f), $63836(\mathrm{u}), 63837(\mathrm{~m})$; ANSP 17982 (m); CA 7845 (f); CU 9155 (m); NCSM 28006 (m); UF 14345 (f); USNM 297451 (f). Monmouth: UMMZ 79423 (f), 79424 (f), 79425 (3m:3f). Ocean: AMNH 64028 (f); NCSM 61473 (m), 61474 (m). 
Passaic: AMNH 85877 (m), 87492 (m), 87493 (f), 91919 (m), 91920 (f), 97249 (f), 97656 (f), 101272 (f), 107310 (m). Warren: AMNH 110185 (m), 139355 (f); LSUMZ 7481 (f).

New York.-Cattaraugas: NYSM 2630 (f). Chemung: CU 3657 (m), 4361 (m), 4362 (f), 4363 (f), 4364 (f); NYSM 2626 (f). Columbia: NYSM 2625. (f). Delaware: LSUMZ 38641 (m). Dutchess: FMNH 38587 (m); KU 61312 (f); NYSM 2902 (f), 2903 (m), 2940 (m), 2941 (f); UF 116100 (m). Essex: ANSP 19864 (m), 19865 (f); NYSM 2129 (m). Montgomery: NYSM 2622 (m). Ontario: CU 3416 (f), 13057 (f); NYSM 2623 (m), 2627 (m); Orange: CU 9193 (m), 9195 (f), 9196 (m), 9197 (f), 9198 (f), 9199 (f), 9200 (f), 9201 (f), 13369 (m); NYSM 2893 (m); USNM 330018 (f), 523668 (m). Rockland: CA 7392 (m); UMMZ 79427 (2f). Steuben: CU 4392 (f), 13057 (m). Sullivan: NYSM 2892 (m), 3048 (f), 3049 (m); OUVC 1211 (m), 1238 (f); UMMZ 79426 (2m:3f). Ulster: NYSM 2624 (m). Warren: NYSM $1118(\mathrm{~m}), 1119(\mathrm{~m}), 1120(\mathrm{~m}), 1121(\mathrm{~m}), 1122(\mathrm{~m})$, 1123 (f), 1124 (m), 1125 (f), 1126 (f), 1127 (m), 1128 (f), 1129 (f), 1130 (f), 1131 (m), 1132 (m), 1133 (m), 1134 (f), 1135 (m), 1137 (f), 1139 (f), 1142 (f), 1143 (f), 1149 (f), 1150 (f), 1151 (f), 1152 (m), 1153 (f), 1154 (m), 1156 (f), 1159 (f), 1160 (m), 1161 (m), 1162 (m), 1163 (f), 1164 (m), 1165 (f), 1166 (f), 1168 (f), 1170 (f), 1171 (f), 1176 (m), 1177 (f), 1178 (f), 1179 (f), 1180 (m), 1181 (m), 1502 (f), 1503 (f), 1512 (f), 1513 (f), $1514(\mathrm{f}), 1515(\mathrm{f}), 1516(\mathrm{~m}), 1517(\mathrm{~m}), 1518(\mathrm{~m}), 1519(\mathrm{~m}), 1520(\mathrm{~m}), 1521(\mathrm{~m}), 1522$ (m), 1523 (m), 1524 (m), 1525 (m), 1528 (f), 1529 (f), 1530 (f), 1531 (f), 1532 (f), 1533 (f), 1534 (f), 1535 (f), 1536 (m), 1537 (m), 1538 (m), 1539 (m), 2130 (m), 2131 (m). Washington: NYSM 1183 (m). Wyoming: CU 13046 (f). No County: AMNH 24944 (m).

North Carolina.-Anson: NCSM 7869 (f), 9521 (m). Avery: NCSM 14111 (f); UF 8460 (f). Beaufort: NCSM 5731 (f), 11843 (f), 12825 (f), 15025 (m), 15660 (m), 29118 (f), $29373(\mathrm{~m}), 30731(\mathrm{~m})$, Bertie: NCSM 11874 (f), 11875 (f), $11883(\mathrm{~m}), 11884(\mathrm{~m}), 11885$ (m), $11886(\mathrm{~m}), 11887$ (m), 11888 (f), 11889 (f), 11890 (f), 11921 (m), 11922 (m), 11923 (m), 11924 (m), 11925 (f), 11926 (m), 11927 (f), 11928 (f), 11929 (f), 11930 (f), 11931 (f), 11932 (f), 11933 (f), 20977 (m). Bladen: NCSM 5933 (m), 11317 (m), 12061 (m), 14646 (m), 15717 (m), 15718 (f), 15719 (f), 15720 (f), 15721 (f), 15792 (m), 15793 (f), $23914(\mathrm{~m}), 25476$ (f), 31927 (m), 39532 (f), 39662 (f), 39663 (f). Brunswick: NCSM $15064(\mathrm{~m}), 15648(\mathrm{~m})$. Buncombe: NCSM $12857(\mathrm{~m}), 12876(\mathrm{~m}), 24675(\mathrm{~m}), 24871(\mathrm{~m})$. Burke: NCSM 13899 (f), 16070 (m), 53789 (f). Camden: CM 23593 (m); NCSM 37308 (f); ODU 1004 (m). Carteret: NCSM 2338 (m), 2345 (f), 12263 (f), 12266 (f), 29119 (m). Cherokee: CM 6366 (m); UMMZ 80985 (f). Clay: FMNH 45855 (f); NCSM 35445 (f), 35446 (m). Columbus: NCSM $39664(\mathrm{~m})$. Craven: NCSM 52966 (f). Currituck: ODU 1023 (m). Dare: ANSP 30219 (f); CM 22933 (m); NCSM 30832 (m); UF 72644 (f). Davidson: NCSM 9879 (f), 9880 (f), 9881 (f), 9882 (f), 9883 (m), 9884 (m), 9885 (f), 9886 (f), 12875 (m), 31095 (m). Durham: NCSM 24672 (f), 52964 (m), 53778 (f). Graham: NCSM 5084 (f). Granville: NCSM 24260 (f), 24504 (m). Henderson: NCSM 2336 (f), 2337 (m), $2670(\mathrm{~m})$; TU 17052 (f). Hoke: NCSM 8725 (m). Hyde: CA 5484 (m), 5702 (f), 5703 (f); NCSM 10779 (f), 16711 (f), 29417 (m); USNM 234403 (m). Jackson: FMNH $244352(\mathrm{~m})$; NCSM 2334 (m), 2665 (m), 17135 (m), 35635 (f); UF 527 (f), 4528 (f); UMMZ 97571 (m). Jones: NCSM 17150 (f), 17151 (m), 17152 (m), 17153 
(m), $17154(\mathrm{~m}), 17155(\mathrm{~m}), 17156(\mathrm{~m}), 17157(\mathrm{~m}), 17158$ (f), 17159 (f), $17160(\mathrm{f}), 17161$ (f), 17162 (f), 17163 (f), 17164 (f), 17165 (f), 17166 (f), 17167 (f), 17168 (f), 24462 (m). Lenoir: NCSM 30771 (f). Macon: NCSM $14011(\mathrm{~m}), 15322(\mathrm{u}), 17386(\mathrm{~m}), 17387(\mathrm{~m})$, 17388 (m), 19595 (f), 19596 (f), 19597 (f), 19672 (m), 19673 (m), 19674 (m), 19675 (m), 19676 (f), 19677 (f), 19678 (f), 19679 (f), 19691 (m), 19791 (m), 62537 (m); UMMZ 135974 (f). Mecklenburg: NCSM 61877 (f). Mitchell: NCSM 2664 (m), 2666 (m). Montgomery: NCSM 5937 (f), 20173 (f), 29295 (m), 29303 (m), 29974 (f), 30244 (m), $30706(\mathrm{~m}), 60376$ (m), 60420 (f). Moore: NCSM $10229(\mathrm{~m})$. New Hanover: NCSM 37250 (f). Onslow: NCSM 8031 (f), 16656 (m), 24345 (m), 31059 (f), 45882 (m); USNM 345445 (m). Pamlico: NCSM 17146 (m), 52965 (m). Pender: LSUMZ 39988 (f); NCSM 2346 (m), 15926 (f); UMMZ 91954 (m). Perquimans: NCSM 30146 (m). Pitt: NCSM $2341(\mathrm{~m}), 2342$ (f), 2343 (f), 2344 (f), 5774 (m), 5775 (m), 5776 (m), 5777 (m), 5778 (m), 5779 (m), 5780 (f), 5781 (f), 5782 (f), 5783 (f), 5784 (f), 5785 (f), 5786 (f), 5787 (f), 5788 (f). Polk: NCSM 11588 (m), 12894 (m). Randolph: NCSM 11259 (m), 29243 (f), 29390 (f), 30140 (f). Rutherford: NCSM 10920 (m), 29659 (m). Sampson: NCSM 17180 (f), 18164 (m), 19454 (m), 31906 (f). Scotland: NCSM 5178 (m), 5393 (f), 5744 (f), 5745 (m), $5746(\mathrm{f}), 5747(\mathrm{f}), 5748(\mathrm{f}), 5749(\mathrm{~m}), 5750(\mathrm{f}), 5751(\mathrm{~m}), 5752(\mathrm{~m}), 5753(\mathrm{f}), 5754$ (f), $5755(\mathrm{~m}), 5756(\mathrm{~m}), 5757(\mathrm{f}), 8041(\mathrm{~m}), 8326(\mathrm{~m}), 11017(\mathrm{~m}), 12011(\mathrm{f}), 17105$ (f), $17106(\mathrm{~m}), 17107(\mathrm{~m}), 17108(\mathrm{~m}), 17109(\mathrm{~m}), 17110(\mathrm{~m}), 17111(\mathrm{~m}), 17112(\mathrm{~m}), 17113$ (f), 17114 (f), 17115 (f), 17116 (f), 17117 (f), 17118 (f), 17119 (f), 17120 (f), 21808 (f), $21809(\mathrm{~m}), 21810(\mathrm{~m}), 21811(\mathrm{~m}), 21812(\mathrm{~m}), 21813(\mathrm{~m}), 21814(\mathrm{~m}), 21815(\mathrm{~m}), 21816$ (m), 21817 (m), 21818 (m), 21819 (f), 21820 (f), 21821 (f), 21822 (f), 21823 (f), 21824 (f), 21825 (f), 21826 (f), 31954 (m), 60373 (m); UGAMNH 4797 (m); USNM 156804 (f). Stanly: NCSM 2835 (m), 5758 (f), 5759 (f), 5943 (f), 7856 (m), 8035 (f), 8121 (f), 8681 (f), $9576(\mathrm{~m}), 9638(\mathrm{~m}), 9655(\mathrm{f}), 9656(\mathrm{~m}), 9657(\mathrm{f}), 9658(\mathrm{~m}), 9659(\mathrm{~m}), 9660(\mathrm{~m})$, 9661 (m), 9662 (f), 9663 (m), 9664 (f), 9665 (m), 9741 (m), 9772 (f), 9773 (f), 9774 (f), $9775(\mathrm{~m}), 9776(\mathrm{~m}), 9777(\mathrm{f}), 9778(\mathrm{~m}), 9779(\mathrm{~m}), 9780(\mathrm{~m}), 9781(\mathrm{~m}), 9782(\mathrm{f}), 12795$ (m), $15420(\mathrm{~m}), 15645$ (m), 53780 (f). Stokes: NCSM $2633(\mathrm{~m}), 9764$ (m), $36448(\mathrm{~m})$, 39085 (f), 39514 (f), 39947 (m). Surry: NCSM 15363 (f), 15672 (m), 15678 (f), 15679 (m), 17056 (f), 19641 (f), 19642 (f), 19643 (f), 19644 (m). Swain: NCSM 12108 (f), 12109 (m), 12110 (f), 12111 (m), 12112 (f), 12113 (m), 18665 (f); UMMZ 113395 (m). Transylvania: NCSM 2332 (f), 2333 (m), 2637 (m), $2638(\mathrm{~m}), 2639(\mathrm{~m}), 2640(\mathrm{~m}), 2641$ (m), 2783 (f), 2784 (m), 9888 (f), 12234 (m), 17059 (m), 53779 (f), 53783 (f); TU 17895 (3m:2f); UF 4522 (m), 4523 (m); UMMZ 54119 (m). Tyrrell: NCSM 15808 (m), 35225 (m). Wake: NCSM 4214 (m). Wayne: NCSM 2384 (f). Wilkes: NCSM 15532 (f), 19359 (f), 25240 (m). Yancey: UMMZ 54120 (m). No County: CM 36760 (m), 88991 (m); NWU 154 (m), 155 (f); UMMZ 95329 (m).

Ohio.-Athens: OUVC $3916(\mathrm{~m}), 5206$ (m). Pike: INHS 8956 (m). Ross: OUVC 1863 (m), 9002 (m). Scioto: KU 68961 (f); USNM 260817 (f). Vinton: OUVC 2987 (f), 3101 (f), $3104(\mathrm{~m}), 3266(\mathrm{f})$.

Oklahoma.-Bryan: OMNH 27013 (m). Carter: TCWC 77578 (f). Cherokee: UMMZ 109237 (m). Garvin: UTEP 15099 (f), 15100 (m), 15101 (m), $15102(\mathrm{~m}), 15103(\mathrm{~m})$, 15104 (m), 15105 (m), 15106 (m), 15107 (f), 15108 (m), 15109 (m), 15110 (m), 15111 
(m), 15112 (f), 15113 (m), 15120 (f), 15121 (m). Haskell: OMNH 7341 (m). Jefferson: TCWC 77580 (f). Latimer: OMNH 11758 (f); UTEP 15123 (m), 15124 (m). Le Flore: CM 1173 (f); OMNH 16085 (m), 16086 (f), 38076 (m); UF 72645 (m), 116166 (m); UTA 15660 (m), 24746 (m), 26533 (m), 26534 (m), 32247 (f), 32929 (m). Logan: LSUMZ 34723 (m). Love: OMNH 35392 (f). Marshall: OMNH 27020 (f), 27343 (m), 27640 (m), 27649 (f), 28669 (m), 29925 (m), 34317 (f), 34515 (m). McClain: OMNH 3770 (m), 12529 (m). McCurtain: OMNH 25341 (f); UTEP 15122 (f). Murray: UTEP 15125 (f), 15126 (m), 15127 (m). Noble: LSUMZ 35995 (m); UTEP 15119 (f). Payne: BEECS 383 (m); UTEP 15114 (f), 15115 (m), 15116 (m), 15117 (f), 15118 (f). Pittsburg: TCWC 77579 (f). Pontotoc: UTEP 15128 (f), 15129 (m), 15130 (m). Sequoyah: OMNH 38211 (m). Washington: UMMZ 116634 (m).

Pennsylvania.-Berks: CM 113442 (f). Blair: CM 114640 (m). Cambria: CM 1098 (f), 1370 (m). Cameron: CA 3633 (m); CM 19939 (m), 61017 (m); UF 52702 (f), 52703 (f). Centre: CM 5201 (m), 5202 (m), 111779 (f), 111781 (f). Clinton: CM 34885 (m), 34886 (f), 34887 (f), $37576(\mathrm{~m}), 37577$ (m); UMMZ 79421 (f). Cumberland: CM 11351 (m), 11352 (f), 11353 (f), 27373 (f). Elk: CM 9404 (f). Fayette: CM 20217 (f). Forest: CM 23934 (m), 23935 (f), 31337 (m), 40181 (m). Indiana: CM 34696 (f); Jefferson: CM 22632 (m); Lackawanna: CM 92067 (m); Lebanon: UMMZ 122536 (m); Lycoming: CM $7564(\mathrm{~m}), 7565(\mathrm{~m}), 7566(\mathrm{~m})$. McKean: CM $21926(\mathrm{~m}), 21927(\mathrm{~m}), 21966(\mathrm{~m}), 22622$ (m), $22623(\mathrm{~m}), 22624(\mathrm{f}), 22625(\mathrm{~m}), 22626(\mathrm{~m}), 22627(\mathrm{~m}), 22628(\mathrm{f}), 22629(\mathrm{~m})$, 22630 (f), 22631 (f), 48053 (m). Monroe: CM 9680 (m), 22250 (f). Northumberland: USNM 252644 (m). Pike: CM 30564 (f), 33446 (f). Schuylkill: CM 26167 (m).

Somerset: CM 1793 (f). Sullivan: CM 7598 (f). Susquehanna: CM 91963 (f), 91964 (m), 91965 (f), 91966 (m), 91967 (u), 91968 (f), 91969 (m), 91990 (m), 92027 (m), 92042 (m), 92045 (m), 92068 (f), 92129 (m); LSUMZ 38488 (f), 38642 (f). Tioga: CM 70257 (f), 91677 (f). Venango: CA 6869 (f), 6870 (f), 6871 (m), 6872 (m), 6873 (f), 6874 (f), $6875(\mathrm{f}), 6876(\mathrm{~m}), 7481(\mathrm{~m}), 7482(\mathrm{~m}), 7483(\mathrm{f}), 7484(\mathrm{~m}), 7485(\mathrm{~m}), 9432(\mathrm{f}), 9433$ (f), 9434 (f), 9435 (f), 9436 (f), 9437 (m). Warren: CM 23609 (f), 23610 (f), 23611 (f), 23612 (m), $23613(\mathrm{~m}), 23614(\mathrm{~m}), 23615(\mathrm{~m}), 23616$ (f), $23617(\mathrm{~m}), 23618(\mathrm{~m}), 33679(\mathrm{f})$, 33680 (f); UMMZ $69192(\mathrm{~m}), 69193(\mathrm{f}), 72261(\mathrm{~m}), 72262(\mathrm{~m})$. Westmoreland: CM 1987 (f), 6434 (f), 6435 (m), 6436 (f), 6437 (f), 6438 (f), 6439 (f), 6440 (f), 6441 (m), 35706 (f), 36574 (f). Wyoming: CM 34902 (m), 91445 (f), 91446 (f), 91447 (f), 91465 (m), $91482(\mathrm{~m}), 91483(\mathrm{f}), 91484(\mathrm{~m}), 91501(\mathrm{f}), 91512(\mathrm{f}), 91513(\mathrm{~m}), 91582(\mathrm{~m}), 91583$ (m), 91606 (f), 91607 (m), $91608(\mathrm{~m}), 91609(\mathrm{~m}), 91610(\mathrm{~m}), 91611$ (f), 91612 (f), 91613 (f), 91861 (m), 91951 (f), 92016 (f), 92017 (f), $92018(\mathrm{~m}), 92020(\mathrm{~m}), 92025$ (m), 92026 (f), 92050 (f), 92051 (f), 92052 (f), 92053 (f), 92054 (m), 92055 (f), 92056 (m), 92057 (f), 92063 (f), 92064 (m), 92065 (f), $92066(\mathrm{~m}), 92069$ (f), 92079 (m), 92080 (m), 92081 (f), 92082 (f), 92083 (m), 92084 (m), 92085 (m), 92086 (f), 92087 (m), $92088(\mathrm{~m}), 92089$ (f), 92090 (m), 92091 (f), 92092 (f), 92093 (m), 92094 (f), 92095 (f), 92096 (m), 92097 (m), 92098 (m), 92099 (f), 92100 (m), 92101 (f), 92102 (m), 92103 (f), 92104 (f), 92105 (m), 92106 (m), 92107 (f), 92108 (f), 92109 (m), 92110 (f), 92111 (m), 92112 (f), 92113 (f), 92114 (f), 92115 (m), 92116 (f), 92117 (f), 92118 (f), 92119 (m), 92120 (m), 92121 (f), $92122(\mathrm{~m}), 92123(\mathrm{f}), 92124(\mathrm{~m}), 92125(\mathrm{~m}), 92126(\mathrm{~m}), 92127(\mathrm{~m}), 92157(\mathrm{~m})$, 92182 (m); LSUMZ 9149 (f), 9150 (f), 9385 (f), 9386 (m), 9387 (f), 9388 (m), 9389 (f), 
9390 (f), 42780 (m), 42781 (f). No County: BMNH 13567 (f); CM 9024 (m), 9025 (m), 9026 (f), 34919 (m), 34920 (m), 34921 (f), 34922 (m), 34923 (f), 34924 (f), 34925 (f), 34926 (f), 34927 (f), 34928 (f), 34929 (m), 34930 (f); UF 21687 (f), 21688 (f).

South Carolina.-Aiken: ANSP 35750 (m); FMNH 245298 (m), 245299 (m), 245300 (m); KU 197259 (f), 197260 (m); NCSM 8221 (m); UGAMNH 4769 (f), 4784 (m), 4788 (m), 4796 (m), $4798(\mathrm{~m}), 22830(\mathrm{~m}), 22853(\mathrm{~m})$; USNM 267102 (m). Aiken/Bamwell: UMMZ 129070 (f), 129071 (f). Allendale: AMNH 113038 (m). Barnwell: ANSP 35751 (m); KU 197261 (m); UF 16022 (f); UGAMNH 4789 (m). Beaufort: AMNH 88333 (f); LSUMZ 45387 (m). Charleston: LSUMZ 41524 (f). Clarendon: USNM 314887 (m). Colleton: BEECS 263 (m); UMMZ 136851 (m). Dorchester: AMNH 114041 (f); UMMZ129492 (f). Greenville: UMMZ 79417 (f). Hampton: AMNH 8357 (f); CA 7397 (f); USNM 49958 (m). Jasper: AMNH 97641 (m); BEECS 259 (m), 261 (f), 267 (m), 268 (f); UF 116101 (f); UGAMNH 21403 (m); USNM 218911 (m), 330019 (f), 348514 (f). Lexington: CA 4464 (f), 5450 (m), 17824 (m), 18399 (f); CM 9130 (f), 9132 (m); UMMZ 79413 (m), 79414 (m), 79415 (f), 79416 (f), 122333 (f), 122334 (f). Oconee: NCSM 52967 (m). Orangeburg: UMMZ 199959 (f). Sumter: FMNH 52986 (f).

Tennessee_-Benton: APSU 2768 (m); MUSU 1414 (f). Blount: LSUMZ 43952 (m). Cheatham: APSU $3274(\mathrm{~m})$. Claiborne: CA $13875(\mathrm{~m})$. Cumberland: CA $17389(\mathrm{~m})$, 17498 (f), 18144 (f), 18314 (f). Davidson: APSU 3171 (f). Dekalb: NCSM 8190 (m), 8199 (m); SIUC 3748 (m), 3749 (f). Dickson: APSU 2158 (m). Hickman: APSU 3210 (f). Houston: APSU 3011 (f), 3038 (m), 4355 (m). Humphreys: APSU 2151 (f). Johnson: UTA 33705 (m). Lawrence: CA 7684 (f); LSUMZ 43967 (f). Lincoln: LSUMZ 43949 (f), 43976 (m). Monroe: UF 10849 (4m:2f), 10850 (f), 10851 (f), 10852 (1m:3f), 10853 (m), 10854 (u), 10855 (1m:2f), 10856 (m). Obion: CA 3928 (f). Overton: NLU 46329 (m). Polk: UF 10848 (f). Putnam: NCSM 8200 (m). Sevier: AMNH 73223 (f); CA 7848 (m); INHS 6638 (m); TU 5222 (m); USNM 174020 (f). Stewart: APSU 551 (m), $658(\mathrm{~m})$, 3231 (6m:3f), 4513 (f); MUSU 1337 (m). Wayne: CA $10106(\mathrm{~m})$. Williamson: APSU 5404 (m); LSUMZ 34753 (m). No County: USNM 466214 (m); UTA 7652 (m).

Texas. -Austin: TCWC 10626 (m). Bowie: UTA $22356(\mathrm{~m})$. Brazos: TCWC 5109 (m), $19080(\mathrm{~m}), 31507(\mathrm{~m}), 36349(\mathrm{~m}), 44118(\mathrm{~m}), 48197(\mathrm{~m}), 63503(\mathrm{~m}), 67318(\mathrm{~m}), 67531$ (m), $70388(\mathrm{~m}), 70407$ (f), $71233(\mathrm{~m}), 71956$ (f), $78714(\mathrm{~m})$. Burleson: TCWC 42399 (f), $69836(\mathrm{~m}), 70517(\mathrm{~m})$. Colorado: TCWC $68456(\mathrm{~m})$. Cooke: UTA $22357(\mathrm{~m})$. Eastland: KU 1643 (f), 1648 (m). Ellis: UTA 22358 (f). Freestone: TCWC 80632 (f); UTA 15315 (m). Gonzales: KU 74708 (m); UMMZ 71355 (2m:2f), $79897(\mathrm{~m})$. Grayson: OMNH 37898 (f); UIMNH 19374 (f); UTA 2104 (f). Grimes: TCWC 18383 (m), 69059 (f). Harris: TCWC $81108(\mathrm{~m}), 81109$ (m), 81111 (f). Jackson: TCWC 66154 (m); UTA 38562 (f). Jefferson: CA 7403 (m). Lavaca: TCWC 63090 (f). Lee: TCWC 30763 (m). Leon: TCWC 22955 (m), 82544 (f). Nacogdoches: CA 2178 (f). Newton: TCWC 48482 (m). Robertson: FMNH $53084(\mathrm{~m})$; TCWC 27530 (m); UMMZ 71335 (m). Taylor: CA 393 (m). Tyler: TCWC 71229 (f), 71245 (m), $71730(\mathrm{~m}), 71957$ (m), 72107 (m), 78711 (f), $78712(\mathrm{~m}), 78713(\mathrm{~m}), 80187(\mathrm{~m}), 82545(\mathrm{~m})$. Victoria: TCWC $1176(\mathrm{~m})$; UMMZ 
143719 (m). Walker: TCWC 71223 (m). Waller: TCWC 14932 (m). Wood: UTA 22359 (m). No County: TCWC 68691 (f).

Vermont.-Rutland: AMNH 67289 (f), 68565 (f). No County: UF 4916 (f).

Virginia.-Albemarle: CM 124552 (m), 146062 (m), 146065 (m), 146163 (m).

Alleghany: CA 4454 (m); UMMZ 79428 (1m:1f). Augusta: CM 146047 (m), 146090 (m), 146182 (f), $146380(\mathrm{~m}), 146437(\mathrm{~m})$; USNM $326084(\mathrm{~m}), 326085(\mathrm{~m}), 326086(\mathrm{~m})$. Bath: USNM $313389(\mathrm{~m}), 326087(\mathrm{~m})$. Bedford: AMNH $129419(\mathrm{~m})$; CM $146283(\mathrm{~m})$, 146287 (f), 146297 (m), 146298 (m), 146307 (m), 146312 (m), 146314 (f), 146318 (f), $146360(\mathrm{~m}), 146364$ (f), $146452(\mathrm{~m}), 146454(\mathrm{~m}), 146459(\mathrm{~m}), 146481(\mathrm{~m}), 146483(\mathrm{~m})$, $146491(\mathrm{~m}), 146509$ (f), $146511(\mathrm{~m}), 146514(\mathrm{~m}), 146518(\mathrm{~m}), 146521(\mathrm{~m})$; USNM 326079 (f), 326080 (f), 326081 (m), 326088 (m). Bland: CM 124961 (m). Botetourt: CM 146455 (m). Botetourt/Bedford: CM 146367 (m), 146487 (f), 146489 (m), 146499 (f). Chesapeake: ODU 1002 (m), 1013 (f), 632819 (m); USNM 326082 (m), 326083 (f). Fauquier: USNM 165593 (f). Giles: AMNH 69251 (f), 73222 (m), 73390 (f), 73427 (f), 129421 (m), 137763 (f); CM 21255 (m); UIMNH 2948 (f); UMMZ 89870 (m); USNM 139619 (m). Greene: CM $146046(\mathrm{~m}), 146153(\mathrm{~m})$; USNM 307992 (m). Highland: USNM 347845 (m), 497348 (m), 517844 (f). Isle of Wight: CM 119979 (f). James City: CM 36497 (f). Loudoun: USNM 139620 (m), 255246 (f). Madison: AMNH 63832 (m); CM 146214 (f), 146259 (f), 146261 (m); USNM 84149 (f), 84150 (m). Madison/Page: CM 34478 (f). Nelson: CM 146162 (m), 146168 (m), 146300 (m); USNM 326089 (f). Newport News: CM 36714 (m); ODU 1003 (m); USNM 313390 (f). Norfolk: CM 136950 (m). Page: CM 146173 (m), 146174 (m), 146184 (f), 146186 (f), 146211 (m), $146218(\mathrm{~m}), 146219(\mathrm{~m}), 146254(\mathrm{~m}), 146258(\mathrm{~m})$; USNM $306719(\mathrm{u})$. Patrick: CM 146399 (m). Prince William: USNM 314211 (f), 314212 (f), 314213 (f). Rappahannock: AMNH 136645 (m), 139356 (f), 139357 (m); CM 146176 (m), 146222 (f), 146262 (m), 146265 (m), 146273 (m). Rockbridge: CM 146292 (m), 146474 (f). Rockingham: CM 54721 (f), 146048 (m), 146050 (f), 146070 (f), 146084 (m), 146140 (m), 146141 (f), $146143(\mathrm{~m}), 146144(\mathrm{~m}), 146145(\mathrm{~m}), 146156(\mathrm{~m}), 146181(\mathrm{~m}), 146232(\mathrm{~m})$; UF 1944 (m), 1945 (f); USNM 326090 (f). Virginia Beach: ODU 1001 (f). Warren: CM 146195 (m), 146221 (f), 146244 (f), 146267 (f), 146270 (m). Warren/Rappahannock: CM 146134 (f), 146137 (f). No County: CM 34405 (f), 146196 (f), 146484 (m); ODU 1000 (m); UF $6846(f)$.

West Virginia.-Boone: WVBS 1421 (m), 1921 (f). Greenbrier: WVBS 3490 (m), 10545 (m), 10546 (f). Kanawha: WVBS 1920 (f). Lincoln: WVBS 2276 (m). Logan: WVBS 2041 (f), 2569 (m). Mercer: WVBS 4511 (m). Mingo: CM 15982 (m). Monongalia: CM 43540 (f). Morgan: CM 7136 (m); USNM 286806 (m). Nicholas: CM 12015 (f), 12060 (f). Pendleton: CM 9835 (f), 9840 (f); USNM 246920 (f); WVBS 3538 (m). Pocahontas: CM 12104 (f); WVBS 2050 (f). Randolph: CM 1456 (m); UMMZ 109348 (f). Wayne: WVBS 2898 (m). Wetzel: WVBS 2950 (f). Wyoming: WVBS 2712 (m). No County: UF 1946 (f), 1947 (m). 
Wisconsin.-Buffalo: BMNH 13526 (f), 13564 (f), 13565 (m), 13566 (f); MPM 23360 (f). Dane: MPM 23525 (m). Grant: CM 70345 (f), 70346 (m). Iowa: MPM 23017 (m). LaCrosse: MPM 23357 (m). Sauk: CA $4452(\mathrm{~m}), 4453(\mathrm{~m})$; FMNH 162945 (m); MPM $81(\mathrm{~m}), 82$ (f), $755(\mathrm{~m}), 2186(\mathrm{~m}), 23359(\mathrm{~m}), 23455(\mathrm{~m})$; UMMZ $55611(\mathrm{~m}), 55612(\mathrm{~m})$, $55613(\mathrm{~m}), 64734$ (f), $79418(\mathrm{~m}), 79420$ (f), 79419 (3m:1f). Vernon: MPM 2294 (f), 26564 (m). No County: BMNH 234 (m); CM 31817 (m). 


\section{APPENDIX II \\ MORPHOLOGICAL CHARACTERS}

The characters employed in this study are defined below and their abbreviations are provided. All morphometric characters were measured in millimeters. Gloyd (1940) employed characters 1-10,17, 21, 28, 29, and 30. Pisani et al. (1973) used characters 110, 17, 21, 29, and 30. Brown and Ernst (1986) used characters 1-10, 16, 21, 26, 28, 29, 30, and 32. Gloyd (1940) and Pisani et al. (1973) recorded the equivalent of transverse elements (17) as a composite of body crossbands (16) and body blotches (11-15), but they were not explicit about their criteria for counting body blotches and bands.

\section{Meristic Characters of the Scutellation}

1. Anterior Dorsal Scale Rows (ADS): Number of anterior dorsal scale rows one head length (HLM) from the supraoccipital bone.

2. Midbody Dorsal Scale Rows (MDS): Number of dorsal scale rows at midbody.

3. Posterior Dorsal Scale Rows (PDS): Number of dorsal scale rows one head length (HLM) anterior to the cloaca.

4. Ventral Scales (VS): Number of ventral scales counted according to the method of Dowling (1951).

5. Subcaudal Scales (CS): Number of subcaudal scales.

6. Divided Subcaudal Scales (DCS): Number of divided subcaudal scales, both anterior and posterior.

7. Left Supralabial Scales (LSL): Number of left supralabials.

8. Right Supralabial Scales (RSL): Number of right supralabials.

9. Left Infralabial Scales (LIL): Number of left infralabials.

10. Right Infralabial Scales (RIL): Number of right infralabials.

\section{Meristic Characters of the Pattern}

The anteriormost pattern element consisted of a pair of elongate postcranial blotches that extended onto the neck. Aligned body blotches consisted of ventrolateral and dorsolateral blotches that were in line with each other to form a transverse element. Ventrolateral blotches that occurred between aligned ventrolateral blotches were only counted if they 
were the same size as, or larger than, the aligned ventrolateral blotches. Smaller dark markings that occurred between aligned blotches were not counted.

11. Dorsal Midline Blotches (DMB): Number of dark body blotches (rounded markings) and dark transverse markings (bands or chevrons) that intersected the central scale row at the dorsal midline, counted from the head to the anal plate. Y-bands and Ubands (see 19-20, below) that intersected the dorsal midline in two places were counted as two DMB. Y-bands and U-bands that arched continuously over the dorsal midline were counted as one DMB. Dorsal midline blotches that were obscured by a middorsal stripe were counted as one DMB.

12. Left Dorsolateral Blotches (LDB): Number of dark body blotches and dark transverse markings along the left dorsolateral region, counted three scale rows left of the dorsal midline, from the head to the anal plate.

13. Right Dorsolateral Blotches (RDB): Number of dark body blotches and dark transverse markings along the right dorsolateral region, counted three scale rows right of the dorsal midline, from the head to the anal plate.

14. Left Ventrolateral Blotches (LVB): Number of dark body blotches and dark transverse markings along the left ventrolateral region, counted three scale rows above edges of ventral scales, from the head to the anal plate.

15. Right Ventrolateral Blotches (RVB): Number of dark body blotches and dark transverse markings along the right ventrolateral region, counted three scale rows above edges of ventral scales, from the head to the anal plate.

16. Body Crossbands (BCB): Number of complete body crossbands (dark bands or chevrons) uninterrupted by one or more scales of ground color, counted from the head to the anal plate (Brown and Ernst 1986). Y-bands were counted as one BCB.

17. Transverse Elements (TE): Number of aligned blotches, fused blotches, and body crossbands counted simultaneously along both sides of the body from the head to the anal plate. One TE consisted of a single dorsolateral blotch (see 12-13) aligned with or fused to a single ventrolateral blotch (see 14-15). Transverse elements from opposite sides were counted together if they were aligned or fused. Single dorsolateral elements aligned or fused with two ventrolateral elements were counted as two TE. Alternating half bands from opposite sides were counted separately. Ybands and U-bands were recorded as two TE. Elements were considered aligned if a line drawn along the margin of a TE perpendicular to the dorsal midline intersected with a corresponding TE on the same or opposite sides of the body.

18. Half Bands (HB): Number of body crossbands that were limited to one side of the body, counted from the head to the anal plate. 
19. U-Bands (UB): Number of U-shaped bands, counted from the head to the anal plate. U-bands typically lay entirely on one side of the body, sometimes extending to, and running along, the dorsal midline.

20. Y-Bands (YB): Number of Y-shaped bands, counted from the head to the anal plate. $\mathrm{Y}$-bands typically consisted of a single band on one side of the body that is connected to two bands on the opposite side.

21. Tail Crossbands (TCB): Number of crossbands on the tail, counted from the anal plate to the basal rattle segment. Half bands and complete bands were included in TCB counts.

22. Band Length (BL): Band length was measured as the maximum number of scales along the longitudinal body axis. Band length was averaged from three body crossbands at midbody. Highly irregular or fused bands were not counted.

23. Band Spacing (BS): Band spacing was measured as the number of scales along the longitudinal body axis between the midlines of two body crossbands. The number of scales was counted in the scale row on either side of the dorsal midline scale row (scale row 11 or 12 , depending on MDS). The value for band spacing was averaged from three band spacings measured at midbody. Band spacings of highly irregular or fused bands were not counted. Note that BS measured the distance from the midline of one band to that of the next band, and not simply the distance of ground color between adjacent bands.

24. Middorsal Stripe Width (MSW): Width of the middorsal stripe was measured as the number of scales along the transverse body axis, at the anterior third of SVL.

\section{Coloration Characters}

25. Melanin Density (MD): Melanin density in the ground color was coded at midbody as follows: Low = groups of melanophores were absent or were diffuse and widely scattered at different distances from each other; Medium = groups of melanophores were concentrated in a uniform pattern but were visibly separate from each other; High = groups of melanophores were dense, highly concentrated, and produced mottling and/or black patches; Melanistic = ground color was solid black or nearly so along the entire dorsal and lateral surfaces, with only weak traces of a remnant pattern. Melanin density was the most rigorously applied measure of pigmentation, and was independent of ground color (see 26) and head color (see 27), except for specimens with melanistic melanin density, which were classified as also having black ground/head color.

26. Ground Color (GC): Ground color was the dominant background color at midbody and was coded as Light, Moderate, Dark, or Black. Ground color was independent of melanin density (see 25), except for specimens in which dense groups of 
melanophores obliterated ground color with black pigmentation; thus, black ground color corresponded to high and melanistic melanin densities.

27. Head Color (HC): Head color was the dominant color of the dorsal surface of the head and was coded as Light, Moderate, Dark, or Black. Head color was independent of melanin density (see 25), except for specimens in which dense groups of melanophores obliterated head color with black pigmentation; thus, black head color corresponded to high and melanistic melanin densities.

28. Postocular Stripe (POS): Postocular stripe was coded as: Absent $=$ postocular stripe absent; Light = postocular stripe with light pigment on all scales; Partial Dark = postocular stripe with light pigment on central areas or posterior edges of scales; Solid Dark = postocular stripe uniformly darkly pigmented.

\section{Morphometric Characters of the Body}

29. Snout-Vent Length (SVL): Snout-vent length was measured to the nearest $1 \mathrm{~mm}$ from the tip of the rostrum to the posterior edge of the anal plate by extending the specimen along a metric tape.

30. Tail Length (TL): Tail length was measured to the nearest $1 \mathrm{~mm}$ from the posterior edge of the anal plate to the posterior edge of the basal rattle segment by extending the tail along a metric ruler.

31. Maximum Body Circumference (MBC): Maximum body circumference was measured to the nearest $1 \mathrm{~mm}$ in the midbody region, at the point of largest circumference, using a flexible metric tape.

\section{Morphometric Characters of the Head}

32. Head Length to the Mandible (HLM): Head length to the mandible was measured to the nearest $0.1 \mathrm{~mm}$ from the tip of the rostrum to the posterior end of the left mandible parallel to the longitudinal axis of the body.

33. Head Length to the Rear of the Supraoccipital Bone (HLS): Head length to the supraoccipital bone was measured to the nearest $0.1 \mathrm{~mm}$ from the tip of the rostrum to the posterior edge of the supraoccipital bone.

34. Head Width (HW): Head width was measured to the nearest $0.1 \mathrm{~mm}$ at the level of maximum distance between the jaws of each specimen.

35. Quadrate Length $(\mathrm{QL})$ : Quadrate length was measured to the nearest $0.1 \mathrm{~mm}$ from the dorsal end to the ventral end of the left quadrate. 
36. Mandible Length (ML): Mandible length was measured to the nearest $0.1 \mathrm{~mm}$ from the anterior end to the posterior end of the left mandible. This measure differed from HLM in being measured parallel to the mandibular bone itself, rather than to the longitudinal body axis. 


\section{APPENDIX III}

\section{REGIONAL CLASSIFICATION USED IN THIS STUDY}

Regions used in this study are defined below and their abbreviations are provided.

\begin{tabular}{ll} 
ALMI & Alabama and eastern Mississippi \\
APPS & Southern Appalachian Mountains in northern Georgia and Alabama \\
AR & Arkansas \\
CTMA & Connecticut and Massachusetts \\
FL & Florida \\
GA & Georgia \\
IAS & Southern Iowa \\
ILIA & Northwestern Illinois and eastern Iowa \\
ILMO & Southern Illinois and southeastern Missouri \\
ILW & Western Illinois \\
INKY & Southern Indiana and central Kentucky \\
KSNE & Eastern Kansas and southeastern Nebraska \\
KYTN & Western Kentucky and western Tennessee \\
LAMI & Southern Louisiana and southwestern Mississippi \\
MN & Southeastern Minnesota \\
MO & Missouri \\
NCCP & Coastal Plain of North Carolina and Virginia \\
NCMT & Appalachian Mountains in western North Carolina and eastern Tennessee \\
NCPD & Piedmont of North Carolina \\
NJCP & Coastal Plain of New Jersey \\
NYE & Eastern New York and Passaic County (NJ) \\
NYN & Northern New York and Rutland County (VT) \\
NYPA & Western New York and western Pennsylvania \\
OH & Southern Ohio \\
OK & Oklahoma \\
PAE & Eastern Pennsylvania and Warren County (NJ) \\
SC & South Carolina \\
TNC & Central Tennessee \\
TX & Eastern Texas \\
VAMT & Virginia Mountains, Frederick County (MD), and Morgan County (WV) \\
WI & Southwestern Wisconsin \\
WVKY & West Virginia and eastern Kentucky \\
& \\
\hline
\end{tabular}




\section{APPENDIX IV \\ UNIVARIATE STATISTICS OF MERISTIC DATA FOR MALES}

Mean values, descriptive statistics, and ANOVAs of 24 meristic characters in 32 regions for males. Character abbreviations are defined in Appendix II, and regional abbreviations are defined in Appendix III. For each region, the following data are reported for each character: the $F$. ratio $(F)$, level of significance $(P)$, sample size for that character $(n)$, minimum value (Min), maximum value (Max), mean value (mean), and standard deviation (SD). VA indicates that nonnormality and/or heterogeneous variances prevented testing that character in ANOVA.

\begin{tabular}{|c|c|c|c|c|c|c|c|c|c|c|c|c|c|c|}
\hline Region & $\begin{array}{l}\text { Statistic } \\
F \\
P\end{array}$ & $\begin{array}{r}\text { ADS } \\
12.98 \\
0.0001\end{array}$ & $\begin{array}{r}\text { MDS } \\
\text { VA } \\
\text { VA }\end{array}$ & $\begin{array}{r}\text { PDS } \\
4.71 \\
0.0001\end{array}$ & $\begin{array}{r}\mathrm{VS} \\
13.19 \\
0.0001\end{array}$ & $\begin{array}{r}\text { CS } \\
20.15 \\
0.0001\end{array}$ & $\begin{array}{r}\text { DCS } \\
\text { VA } \\
\text { VA }\end{array}$ & $\begin{array}{r}\text { LSL } \\
5.34 \\
0.0001\end{array}$ & $\begin{array}{r}\mathrm{RSL} \\
6.42 \\
0.0001\end{array}$ & $\begin{array}{r}\text { LIL } \\
9.01 \\
0.0001\end{array}$ & $\begin{array}{r}\text { RIL } \\
7.57 \\
0.0001\end{array}$ & $\begin{array}{r}\mathrm{DMB} \\
\mathrm{VA} \\
\mathrm{VA}\end{array}$ & $\begin{array}{r}\mathrm{LDB} \\
22.11 \\
0.0001\end{array}$ & $\begin{array}{r}\text { RDB } \\
23.25 \\
0.0001\end{array}$ \\
\hline \multirow[t]{5}{*}{ ALMI } & $n$ & 47 & 50 & 50 & 50 & 50 & 50 & 46 & 44 & 47 & 45 & 50 & 50 & 50 \\
\hline & Min & 25 & 23 & 19 & 157 & 23 & 1 & 12 & 12 & 14 & 14 & 22 & 24 & 23 \\
\hline & Max & 33 & 27 & 21 & 170 & 29 & 7 & 16 & 15 & 18 & 18 & 30 & 32 & 32 \\
\hline & Mean & 28.3 & 24.9 & 19.2 & 165.1 & 25.9 & 2.0 & 14.2 & 13.6 & 15.9 & 15.7 & 25.9 & 27.0 & 27.1 \\
\hline & $\mathrm{SD}$ & 1.40 & 0.62 & 0.52 & 2.74 & 1.33 & 1.25 & 0.97 & 0.89 & 1.00 & 0.90 & 1.86 & 1.71 & 1.82 \\
\hline \multirow[t]{5}{*}{ APPS } & $n$ & 11 & 11 & 11 & 11 & 11 & 11 & 11 & 11 & 11 & 11 & 11 & 11 & 11 \\
\hline & Min & 27 & 23 & 19 & 159 & 23 & 1 & 13 & 13 & 15 & 15 & 12 & 23 & 22 \\
\hline & $\operatorname{Max}$ & 30 & 25 & 21 & 166 & 26 & 4 & 15 & 15 & 17 & 17 & 28 & 30 & 29 \\
\hline & Mean & 28.0 & 24.0 & 19.5 & 162.8 & 25.2 & 2.1 & 13.8 & 13.8 & 15.7 & 15.6 & 23.7 & 26.1 & 25.7 \\
\hline & SD & 1.26 & 1.00 & 0.69 & 2.23 & 0.98 & 0.94 & 0.75 & 0.60 & 0.65 & 0.67 & 4.31 & 1.87 & 2.10 \\
\hline \multirow[t]{5}{*}{$\mathrm{AR}$} & $n$ & 21 & 21 & 21 & 21 & 20 & 20 & 20 & 21 & 19 & 21 & 21 & 21 & 21 \\
\hline & Min & 27 & 23 & 18 & 161 & 24 & 1 & 13 & 12 & 14 & 14 & 19 & 21 & 23 \\
\hline & $\operatorname{Max}$ & 31 & 27 & 21 & 170 & 29 & 6 & 15 & 15 & 17 & 17 & 27 & 28 & 29 \\
\hline & Mean & 28.6 & 24.9 & 19.6 & 165.1 & 26.5 & 2.6 & 14.1 & 14.0 & 15.3 & 15.4 & 23.7 & 25.4 & 25.7 \\
\hline & SD & 1.33 & 0.83 & 0.93 & 2.94 & 1.47 & 1.70 & 0.79 & 0.92 & 0.81 & 1.08 & 2.19 & 2.01 & 1.90 \\
\hline
\end{tabular}




\begin{tabular}{|c|c|c|c|c|c|}
\hline$\stackrel{m}{2}$ & $\leadsto \vec{\sim}$ নิ & F & 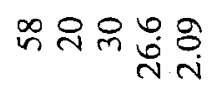 & 유 & 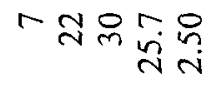 \\
\hline 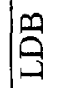 & $\hat{\sim} \vec{\sim} \stackrel{\infty}{\vec{j}} \stackrel{\infty}{-\infty}$ & 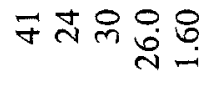 & 旅灾苛 & 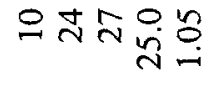 & 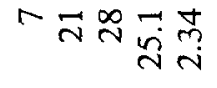 \\
\hline$\sum_{0}^{\infty}$ & 유츄 & 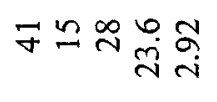 & in ন & 으뮤뮴ำ & $\sim \curvearrowleft \backsim \frac{n}{\sim} \frac{n}{m}$ \\
\hline$\Leftrightarrow$ & 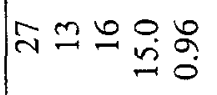 & $\vec{m} \pm \infty \underset{0}{0}$ & 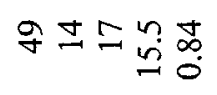 & 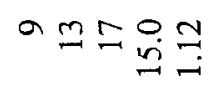 & ヘニニ导 \\
\hline 业 & 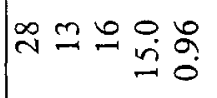 & $\pi \backsim \infty \begin{array}{c}\infty \\
0\end{array}$ & 吋尖芯芯 & 으묘음 & $r m \pm \stackrel{\sigma}{m} \stackrel{\infty}{0}$ \\
\hline$\vec{\omega}$ & $\stackrel{N}{\sim} \cong \underset{m}{\sim} \stackrel{\infty}{0}$ & 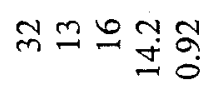 & 足ニニ王术 & $a \cong \pm \stackrel{\infty}{0}$ & $-0 m \underset{=}{\underline{I}}$ \\
\hline की & 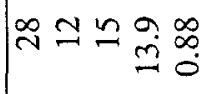 & 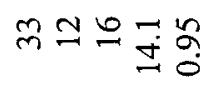 & $N \simeq \mathscr{\pm}$ & 왜ำ & r음요멸 \\
\hline$\tilde{y}$ & ते & $\vec{F}+\infty \dot{m} \bar{g}$ & in or & 을 $\stackrel{\infty}{0}$ & 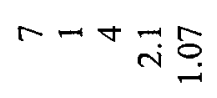 \\
\hline$\tilde{Z}$ & ํㅟำ & অম্ন & 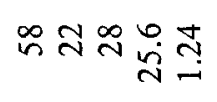 & 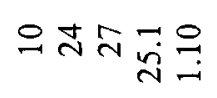 & - \\
\hline$>$ & مิ & 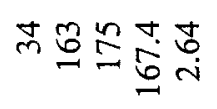 & ถูก & 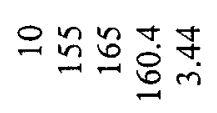 & - \\
\hline مि & 요 & & 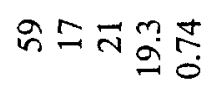 & 으류 & 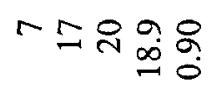 \\
\hline$\overbrace{i}^{2}$ & 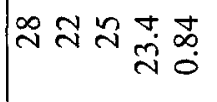 & 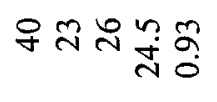 & 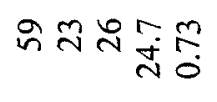 & 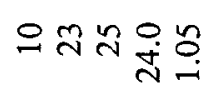 & 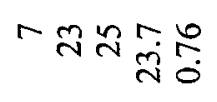 \\
\hline$\hat{z}$ & N & স্রেন্মু & 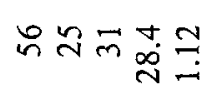 & 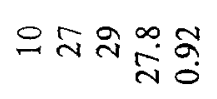 & 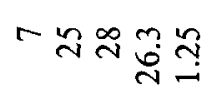 \\
\hline 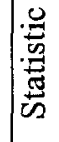 & 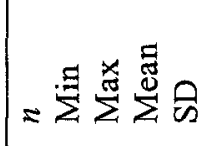 & 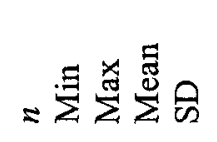 & 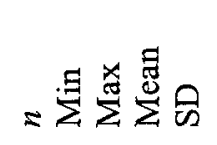 & 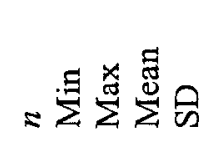 & 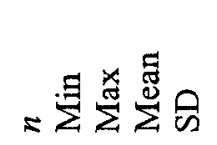 \\
\hline 层 & 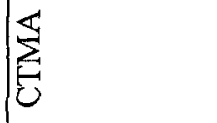 & 宝 & $\mathbb{J}$ & 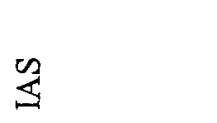 & $\overleftrightarrow{\Xi}$ \\
\hline
\end{tabular}




\begin{tabular}{|c|c|c|c|c|c|}
\hline 貦 & 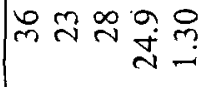 & 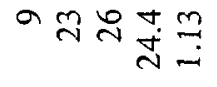 & $\exists \bar{\sim} \stackrel{\infty}{\stackrel{\sim}{\sim}} \underset{\sim}{\sim}$ & 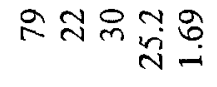 & 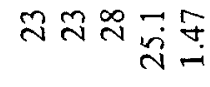 \\
\hline 喤 & 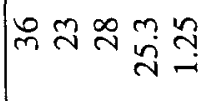 & 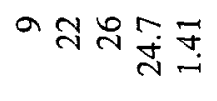 & 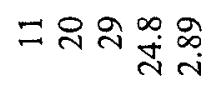 & 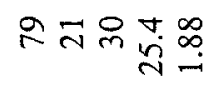 & 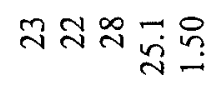 \\
\hline$\sum_{0}^{n}$ & m & $a \approx \approx \underset{\sim}{\sim} \stackrel{\infty}{\infty}$ & $=\stackrel{\sim}{\vec{N}} \underset{\tilde{N}}{\vec{N}}$ & 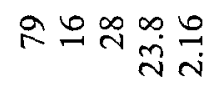 & 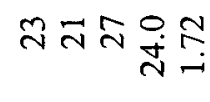 \\
\hline 园 & 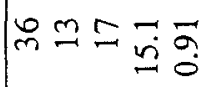 & $\infty む \backsim$ 通 & 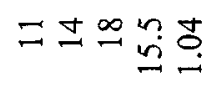 & 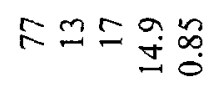 & 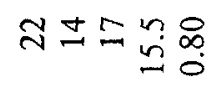 \\
\hline 言 & $m=\Xi$ & 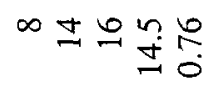 & 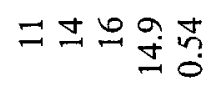 & $R= \pm \underset{ \pm}{ \pm} \sigma$ & 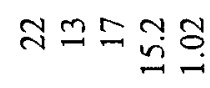 \\
\hline $\overrightarrow{2}$ & $m=0$ & $\infty \simeq \backsim \underset{j}{\infty}$ & $\Rightarrow m \backsim 20$ & 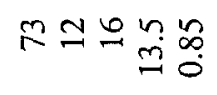 & 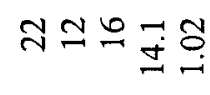 \\
\hline & $m \simeq \because \underset{m}{m} \stackrel{\infty}{=} \stackrel{\infty}{0}$ & - & 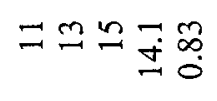 & $\vDash=0 \backsim$ & 즌ㅇ용요 \\
\hline $\begin{array}{l}\mathscr{U} \\
0\end{array}$ & mol & $a-+g \sigma o$ & 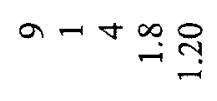 & $\stackrel{\infty}{r}-\underset{i}{\circ}$ & 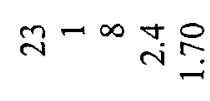 \\
\hline 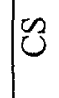 & m & a & a๗ลั & 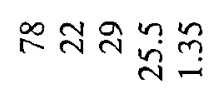 & 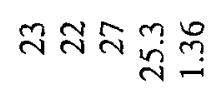 \\
\hline$p$ & 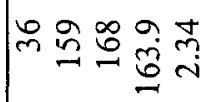 & a & 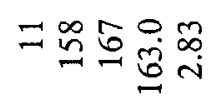 & 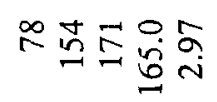 & ส쑈 \\
\hline 告 & 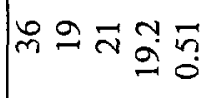 & 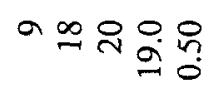 & $=99 \stackrel{9}{9} \dot{0}$ & 믈유 & \\
\hline 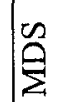 & m & 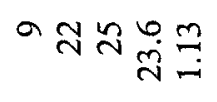 & 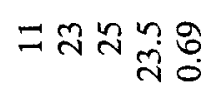 & হNন & 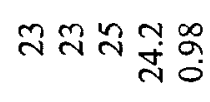 \\
\hline 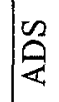 & 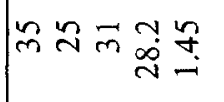 & ๙ & 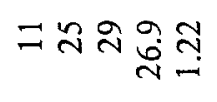 & 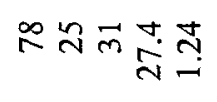 & సి 워 \\
\hline 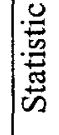 & 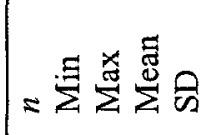 & 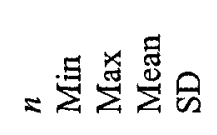 & 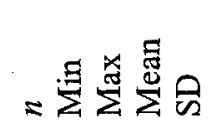 & 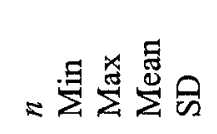 & 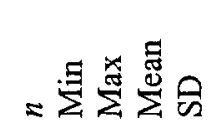 \\
\hline $\mid \begin{array}{l}0 \\
0 \\
0 \\
0 \\
\approx\end{array}$ & 吕 & 学 & 尝 & 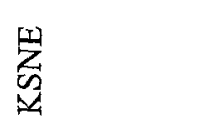 & $\underset{\xi}{Z}$ \\
\hline
\end{tabular}




\begin{tabular}{|c|c|c|c|c|c|}
\hline 寊 & | & 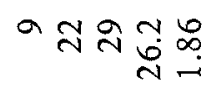 & 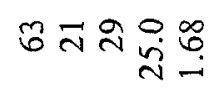 & 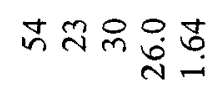 & अํㅠ류류 \\
\hline$\stackrel{m}{\Theta}$ & 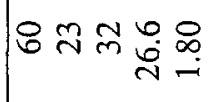 & a $\approx$ స & 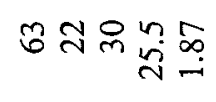 & 苛 & ซึa \\
\hline$\sum_{0}^{n}$ & 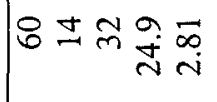 & $a \infty \underset{\sim}{*} \underset{\sim}{n}$ & 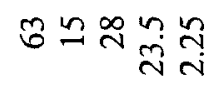 & 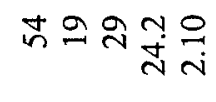 & 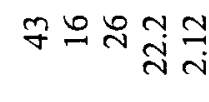 \\
\hline 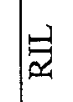 & 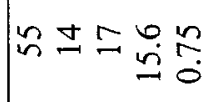 & 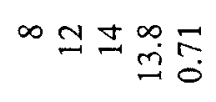 & 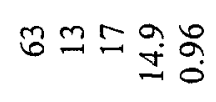 & 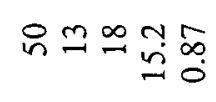 & 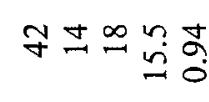 \\
\hline$\stackrel{\vec{\Theta}}{\Theta}$ & 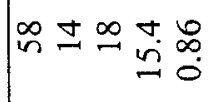 & 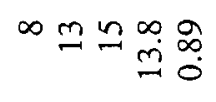 & 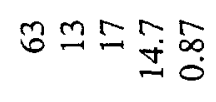 & テッニ㫄合 & 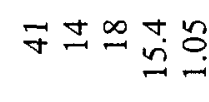 \\
\hline$\stackrel{\overrightarrow{2}}{\approx}$ & 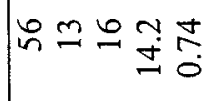 & $\infty a \pm ت \stackrel{n}{\sim}$ & 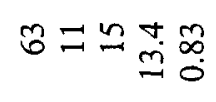 & $g \cong \sim \underset{\dot{g}}{+} \frac{\infty}{\sigma}$ & 쿼유유 \\
\hline $\overrightarrow{\widehat{\Omega}}$ & 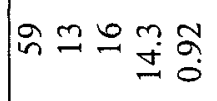 & 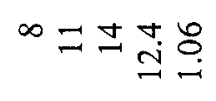 & $\hat{b}=\approx+\dot{g} \hat{\sigma}$ & 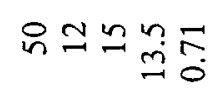 & 워ㅂㅠㅕㅇ \\
\hline 己ֶ & bor & $a-m \infty$ & 이요 & $\stackrel{N}{n}+0 \underset{i}{\infty} \underset{\sim}{\stackrel{0}{0}}$ & $m+r) \stackrel{n}{\rightarrow}$ \\
\hline$\tilde{z}$ & 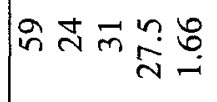 & 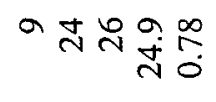 & 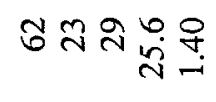 & 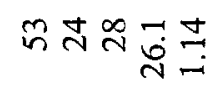 & ชิ่ลั๋ \\
\hline$\stackrel{\infty}{>}$ & مُ & 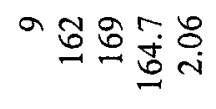 & 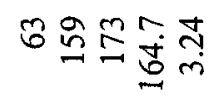 & 芯亘点客管 & Fิn \\
\hline$\hat{a}$ & ธニ & a & 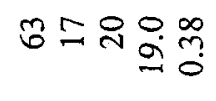 & 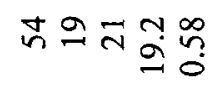 & 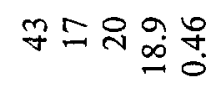 \\
\hline$\stackrel{\infty}{\rho}$ & ธกิ & a๗ & m유껚 & 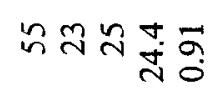 & ซ゙ন ปั \\
\hline 是 & $\infty$ กิ & ส ฝำฺ & 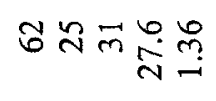 & ஸี & 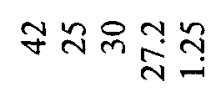 \\
\hline 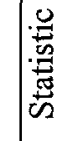 & 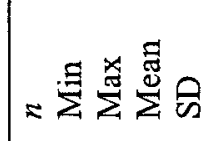 & 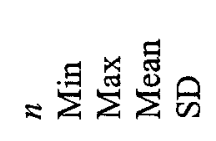 & 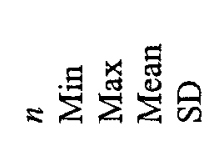 & 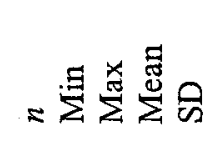 & 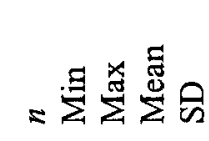 \\
\hline 点 & $\sum_{-3}^{3}$ & 孞 & $\stackrel{O}{\Sigma}$ & $\begin{array}{l}8 \\
\text { O } \\
z\end{array}$ & $\sum_{Z}^{E}$ \\
\hline
\end{tabular}




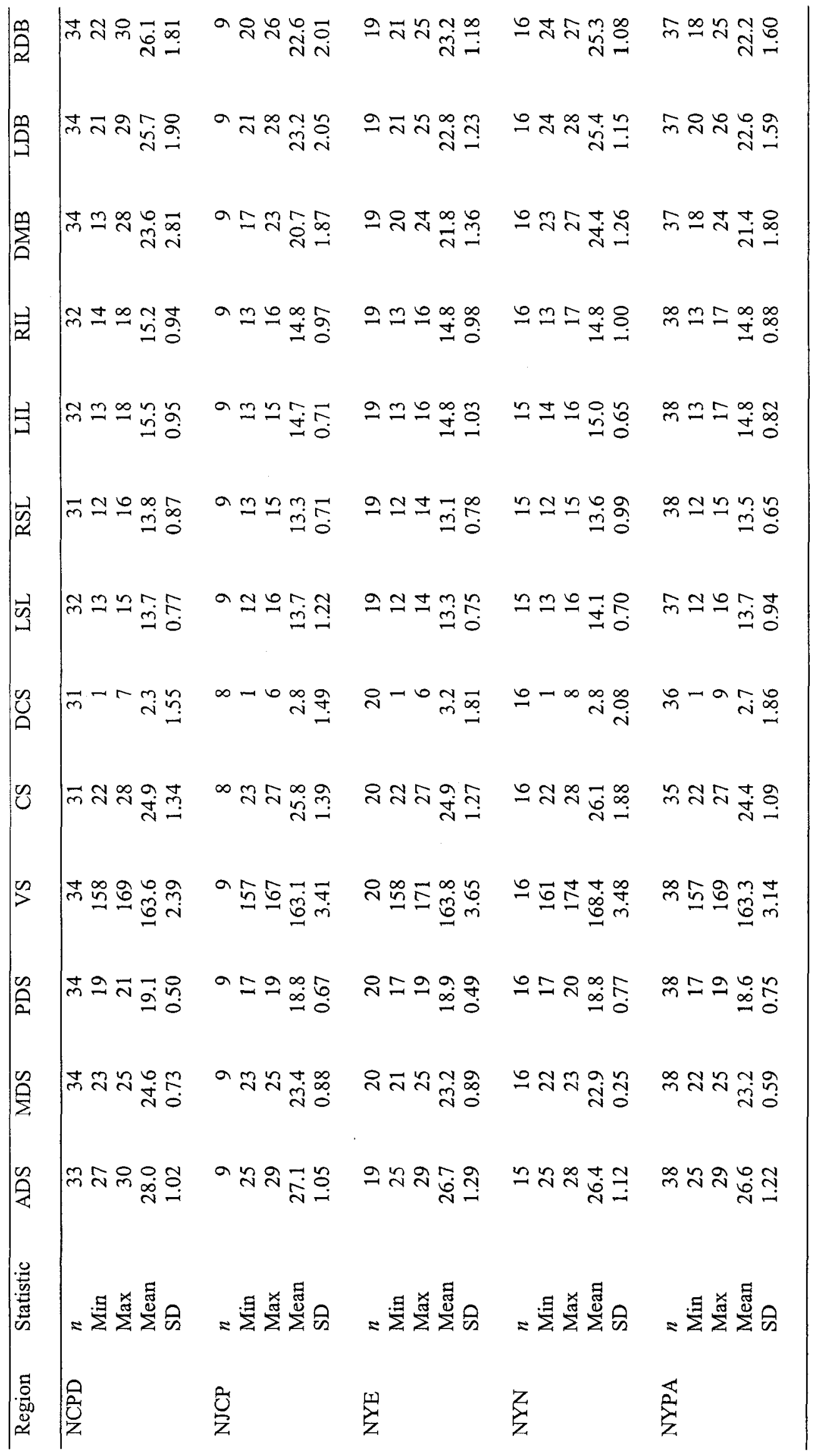




\begin{tabular}{|c|c|c|c|c|c|}
\hline 舀 & 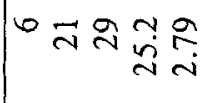 & 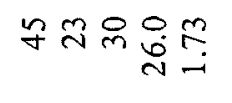 & 苂유 윰 & 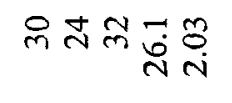 & 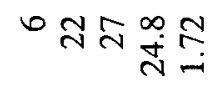 \\
\hline 急 & 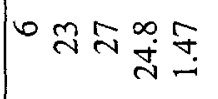 & ๆ & 육유뮴ำ & 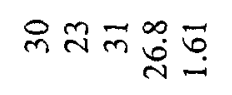 & 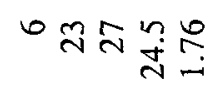 \\
\hline$\sum_{\Delta}^{\infty}$ & log & ケ유속 & lin & 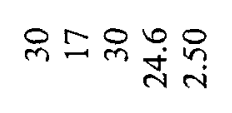 & 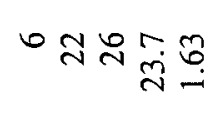 \\
\hline 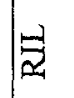 & $0 \pm 0 \tilde{0}$ & 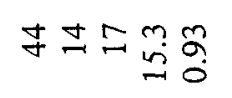 & 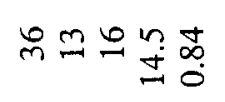 & $\stackrel{\infty}{\sim} \pm I \stackrel{n}{\sim} \underset{\sim}{\infty}$ & 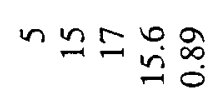 \\
\hline$\exists$ & 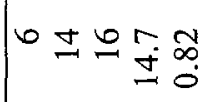 & 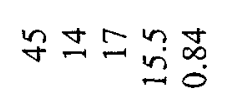 & m & $\tilde{N} \pm \pm \underset{r}{0}$ & $n \simeq \begin{array}{c}* \\
n\end{array}$ \\
\hline 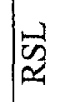 & 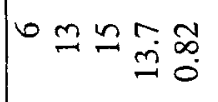 & $\dot{F}=0 \stackrel{\infty}{m} \stackrel{\infty}{g}$ & $\stackrel{m}{m} \backsim \underset{\ddot{m}}{\nabla}$ & 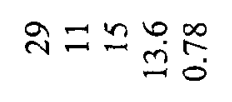 & 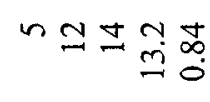 \\
\hline w & om & 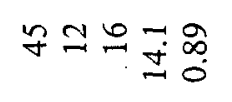 & 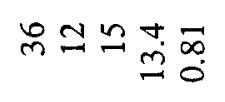 & $\hat{\sim} \approx \backsim \stackrel{\sim}{\sim} \stackrel{\infty}{\circ}$ & $n \simeq \pm \underset{j}{+\infty}$ \\
\hline $\begin{array}{l}0 \\
0 \\
0\end{array}$ & $0-+\underset{i=}{=}$ & $\ddot{q}-\infty \underset{\sim}{\stackrel{m}{\infty}}$ & 品-r兄导 & ते $00 \stackrel{0}{0} \underset{i}{0}$ & $n-m \stackrel{\varphi}{r} \underset{0}{\infty}$ \\
\hline$\tilde{z}$ & 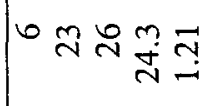 & ঋ & 洜芦 & సిন্ড & $n \vec{\sim} \approx \underset{\sim}{\sim} \stackrel{\square}{\dagger}$ \\
\hline$p$ & 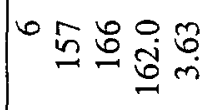 & 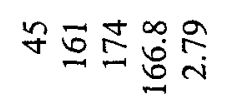 & 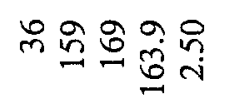 & 욜욤요 & o̊ \\
\hline 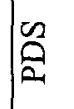 & 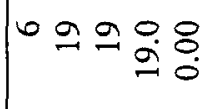 & 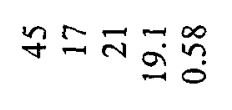 & 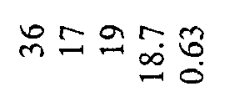 & 음 & 응ㅇㅇㅇㅇㅇ \\
\hline $\mid \begin{array}{c}n \\
\end{array}$ & ๑ & 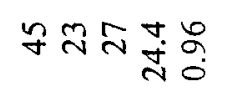 & 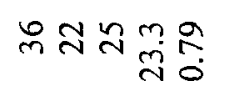 & 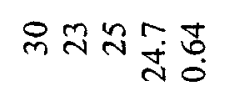 & 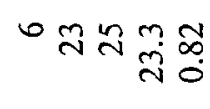 \\
\hline 笔 & o 유 సి & 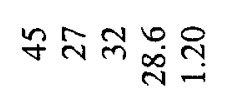 & 육유. & 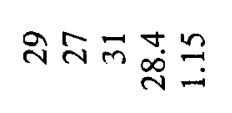 & ○ 규: \\
\hline : & 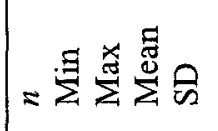 & 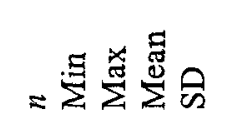 & 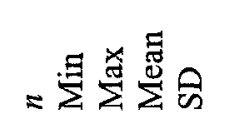 & 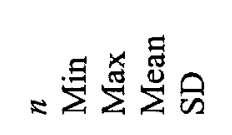 & $=$ 芝希营塄 \\
\hline 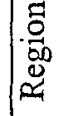 & T: & y̆ & $\sum_{0}^{2}$ & Un & Z \\
\hline
\end{tabular}




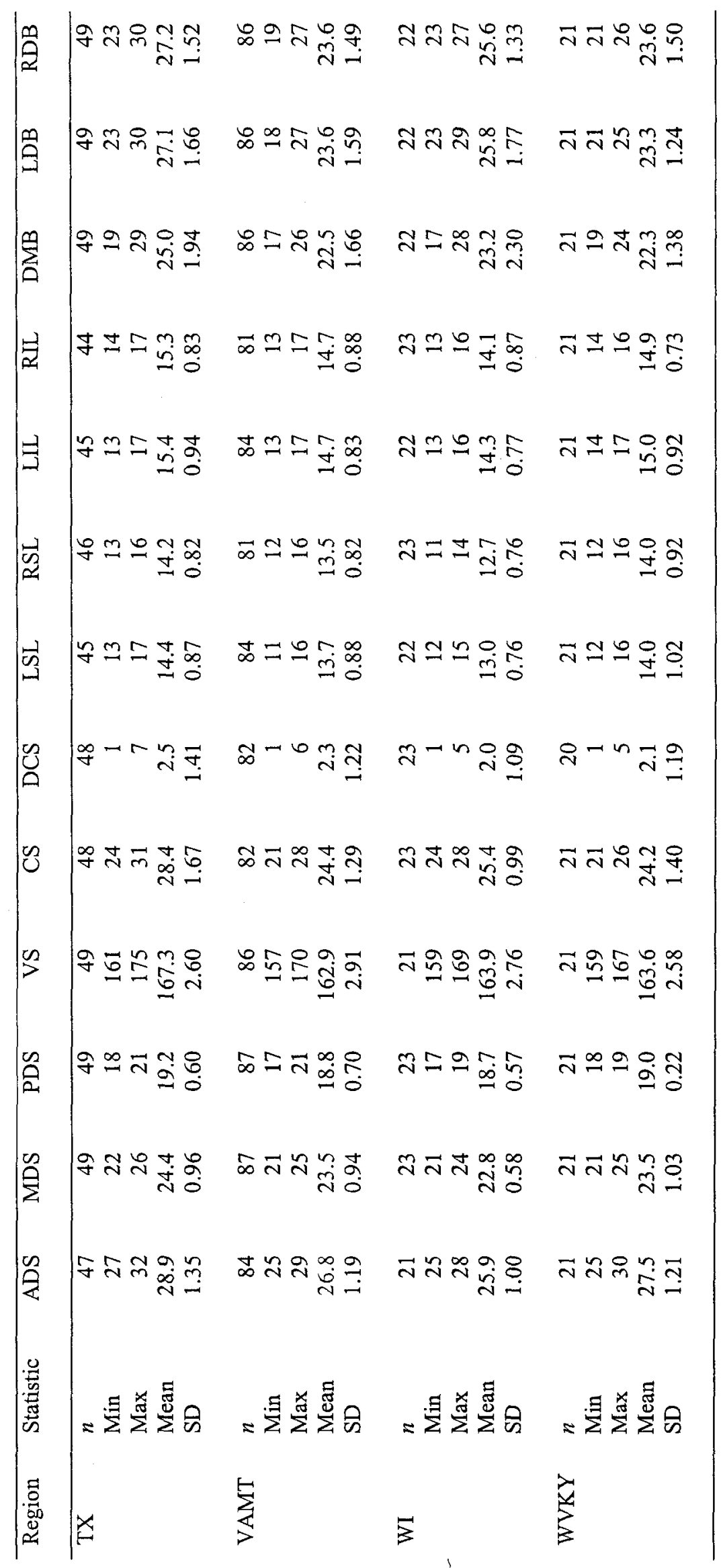




\begin{tabular}{|c|c|c|c|c|c|}
\hline$\sum_{\substack{n \\
i}}^{\substack{>\\
>}}$ & क $N \forall$ लं & aO & 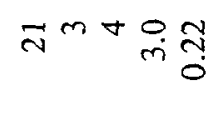 & 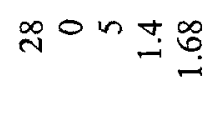 & लेmnन लु \\
\hline صि & 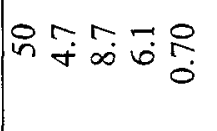 & 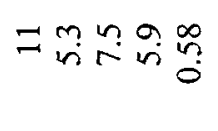 & तี & 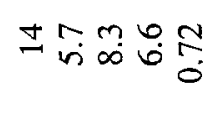 & 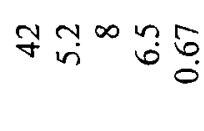 \\
\hline 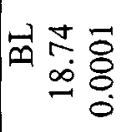 & 品 & $=\underset{\sim}{N} \underset{m}{m} \stackrel{\infty}{\sim} \frac{q}{0}$ & $\vec{\nabla} \sim \overrightarrow{r i} \dot{i}$ & $\hat{N}=\dot{r}$ & 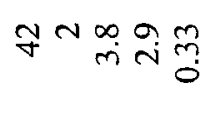 \\
\hline$\ddot{\forall}$ & : & $\Rightarrow 0 n m g$ & $\vec{N}-\operatorname{mn}$ & 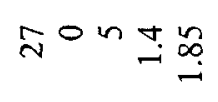 & Yon \\
\hline 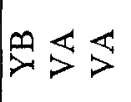 & inom & $=0-\dot{0}$ & $\vec{N} 0+\overrightarrow{0}$ & ñ & 풍- 중 \\
\hline$\stackrel{s}{s}$ & $i_{0} 0-8 \frac{0}{0}$ & $=00: 8$ & $\vec{N} \circ 0: \frac{0}{0}$ & no- 0 용 & $\vec{\nabla}^{0}-\stackrel{0}{0} \frac{0}{0}$ \\
\hline $9 \longleftarrow$ & : & $=0 \infty \sim \underset{\sim}{n} \underset{i}{\infty}$ & $\vec{N}$ 的突冚 & son: $=\frac{n}{n}$ & Fo윰 \\
\hline 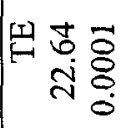 & 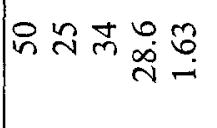 & 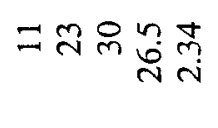 & 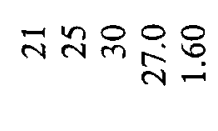 & 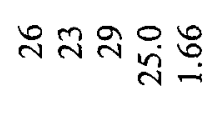 & 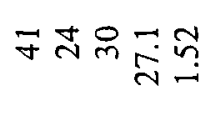 \\
\hline$\bigoplus_{m} \lesssim$ & 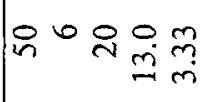 & $=\infty=\underset{a}{a}$ & $\vec{\sim}^{0} \stackrel{\infty}{-} \stackrel{0}{0}$ & $\stackrel{\sim}{\sim} \sim \underset{n}{n}$ & 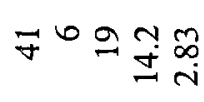 \\
\hline 舟宫 & i̊ & 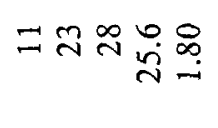 & 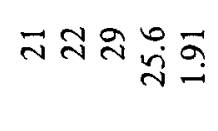 & $\because \vec{\sim} \underset{\sim}{\stackrel{n}{g}}$ & 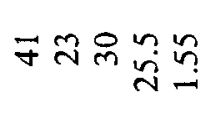 \\
\hline$\left\{\begin{array}{l}n \\
2 \\
0 \\
0 \\
0\end{array}\right.$ & 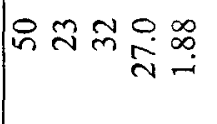 & 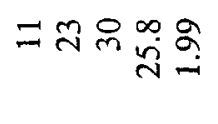 & 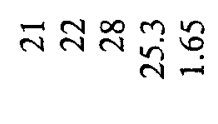 & 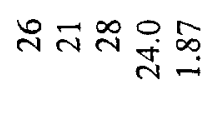 & 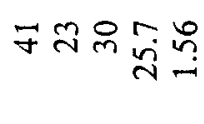 \\
\hline 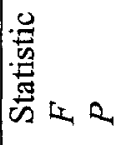 & 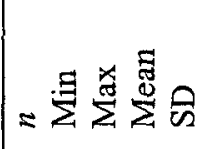 & 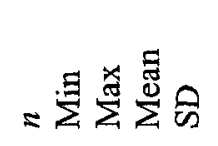 & 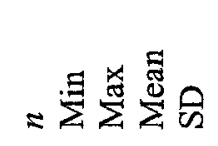 & 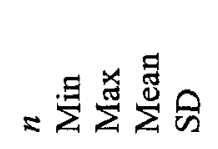 & 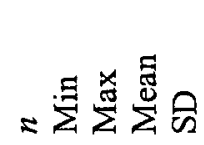 \\
\hline 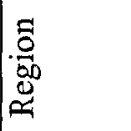 & $\sum_{3}$ & $\frac{n}{2}$ & $\frac{\alpha}{4}$ & $\sum_{\Xi}^{\lessgtr}$ & 鳬 \\
\hline
\end{tabular}




\begin{tabular}{|c|c|c|c|c|c|}
\hline $\mid \begin{array}{l}3 \\
\Sigma\end{array}$ & 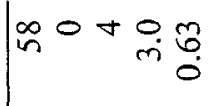 & 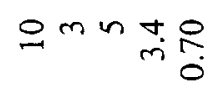 & notrîn & lo 0 的 & a o n Ny m \\
\hline 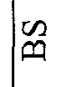 & 象 n? & 아유 & ri r & 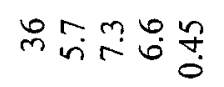 & ann \\
\hline$\omega$ & भिं जिं & ONm nim & $N N \ddot{m} \vec{i}$ & 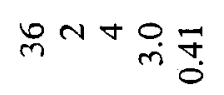 & a Nin \\
\hline$\theta_{\varphi}^{\infty}$ & 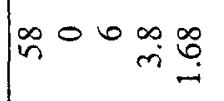 & 응 in & roナ島 & monn & $\infty-\forall \stackrel{i}{\vec{i}}$ \\
\hline$\sum$ & 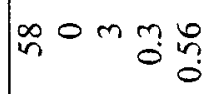 & 음 & NoO & le & a $0-\pi \frac{0}{0}$ \\
\hline$\infty$ & $\infty 0-0 \frac{m}{0}$ & 00008 & No 008 & mo- $\frac{0}{0} \frac{\pi}{0}$ & 90008 \\
\hline$\stackrel{9}{\exists}$ & $\infty \circ \cong \stackrel{\sim}{\sim}$ & 음유윻맘 & - $\circ \stackrel{\infty}{0} \underset{\infty}{\hat{\sigma}}$ & lo $\circ \circ \stackrel{\infty}{\rightarrow} \underset{i}{\sigma}$ & 00098 \\
\hline$\stackrel{\mu}{\theta}$ & m & 유 & 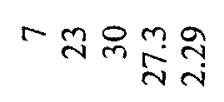 & 足 স্ণ & 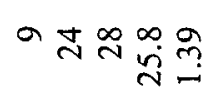 \\
\hline$\ddot{\theta}_{0}$ & $\infty \infty 20 \%$ & 으ㄹㅠㅛ & 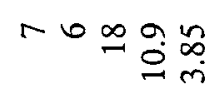 & 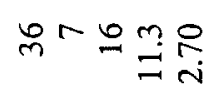 & 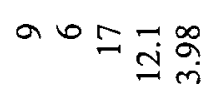 \\
\hline$\sum_{2}^{\infty}$ & 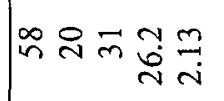 & 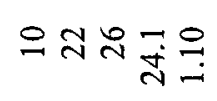 & - & 品 ָ̃ & aتี \\
\hline$\sum_{3}^{\infty}$ & م $\approx$ ন & 읏츄영 & N & 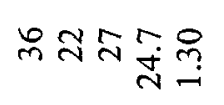 & ดสํี่ \\
\hline 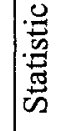 & 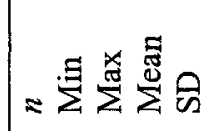 & 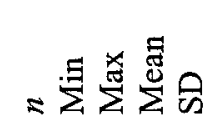 & 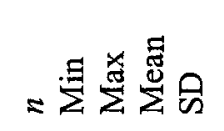 & 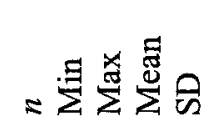 & 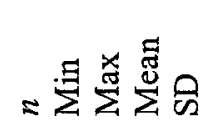 \\
\hline .0 & $\overleftrightarrow{0}$ & 柋 & 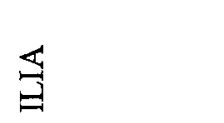 & $\stackrel{\wp}{\sum_{日}}$ & 宣 \\
\hline
\end{tabular}




\begin{tabular}{|c|c|c|c|c|c|}
\hline$\sum_{\substack{3 \\
2}}^{3}$ & $=0+9$. & 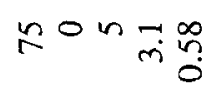 & $\ddot{\lambda} m \dot{r} \vec{j}$ & in- +0 i & a O \\
\hline$\tilde{\omega}$ & $00 \times$ in & 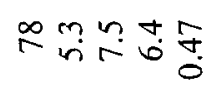 & 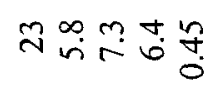 & Bn & aทำฺู \\
\hline$\vec{\infty}$ & 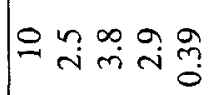 & 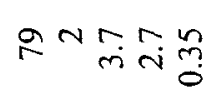 & 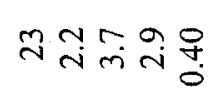 & भิ Nm mị & $a \stackrel{\infty}{-i} \underset{i}{n} \stackrel{m}{0}$ \\
\hline$\bigcup_{i}^{\infty}$ & OO+ & $\stackrel{\infty}{\sim}$ in $\stackrel{a}{\vec{\sigma}}$ & nons & nి०0 & aOnj: \\
\hline 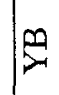 & $00-\overrightarrow{0} \frac{n}{0}$ & rom & moNm & $80-40$ & aON : \\
\hline 9 & 0 & 잉ㅇㅇ & 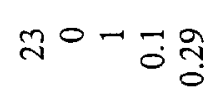 & $80-0 \frac{0}{0}$ & 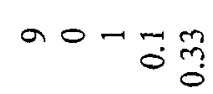 \\
\hline 果 & o의. & R०=N & $\tilde{\sim} \circ \infty \stackrel{\sim}{ָ}$ & $8^{\circ}=\sim \underset{i}{n}$ & a०d \\
\hline 留 & 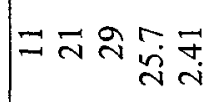 & 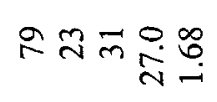 & 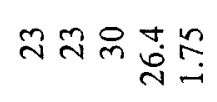 & ৪্ল m̊ & 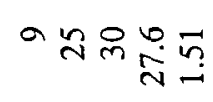 \\
\hline 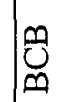 & $=0 \Omega$ & 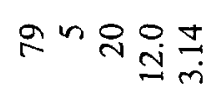 & $\ddot{\lambda}=\exists \stackrel{\infty}{n}$ & $8 n 2 \because \frac{n}{0}$ & 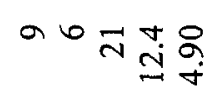 \\
\hline$\sum_{2}^{\infty}$ & $=\bar{\sim} \approx \stackrel{\sim}{\dot{d}}$ & 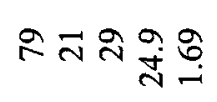 & ก సి유. & В สำ & 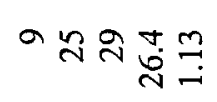 \\
\hline$\sum_{1}^{\infty}$ & $=$ = 유 & 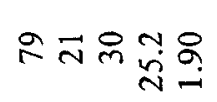 & 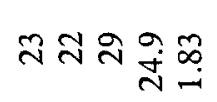 & 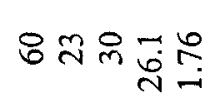 & 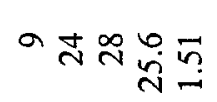 \\
\hline | & 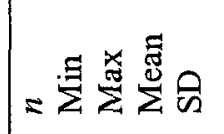 & 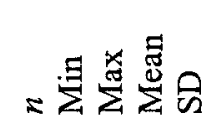 & 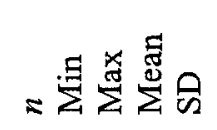 & 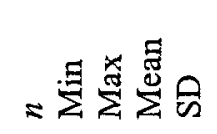 & 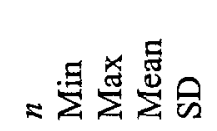 \\
\hline : & 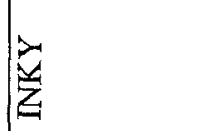 & 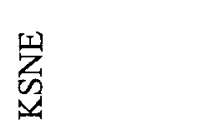 & z & $\sum_{i}^{F}$ & z \\
\hline
\end{tabular}




\begin{tabular}{|c|c|c|c|c|c|}
\hline$\frac{3}{2}$ & 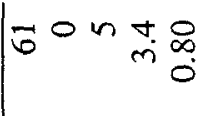 & 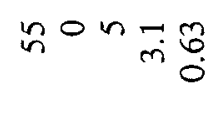 & mon & मे & ao in $\underset{i}{\stackrel{8}{8}}$ \\
\hline$\infty$ & 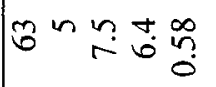 & 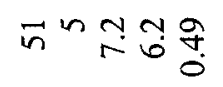 & 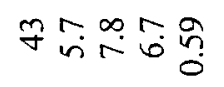 & 芦 & no \\
\hline$\vec{\oplus}$ & B̧त & 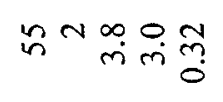 & 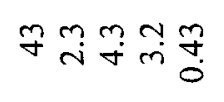 & से & ar \\
\hline 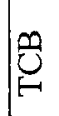 & monn & से०0m & mon & NOONg & lon \\
\hline 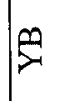 & 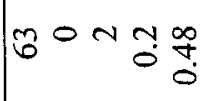 & ñ $\sigma \forall+\frac{t}{0}$ & 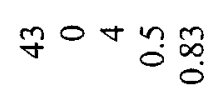 & मे m & aOn? \\
\hline$\frac{m}{s}$ & mor $00 \%$ & $n 0-0 \cdot \frac{m}{0}$ & mo- $: \overrightarrow{0}$ & H০০ & a $000: 8$ \\
\hline 里 & nु० & ñom & 앙요욤 & 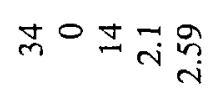 & aOn \\
\hline$\stackrel{\mu}{H}$ & 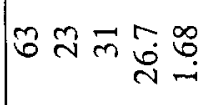 & 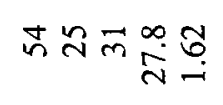 & 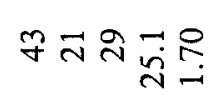 & 芦志究 & 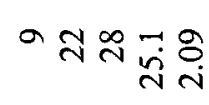 \\
\hline$\underbrace{}_{m}$ & 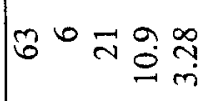 & 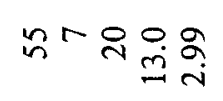 & $\underset{\forall}{m} \vec{N} \underset{=}{=} \frac{0}{m}$ & 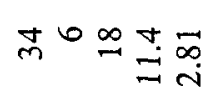 & 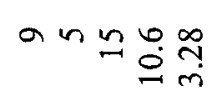 \\
\hline$\sum_{\infty}^{\infty}$ & 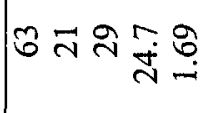 & ฟ & q ন্ণ & 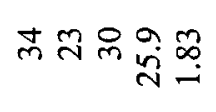 & 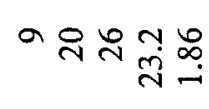 \\
\hline$\sum^{\infty}$ & m유윰ำ & 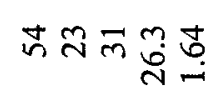 & 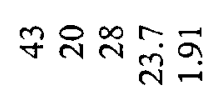 & 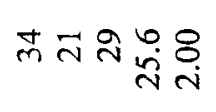 & a సָ \\
\hline | & 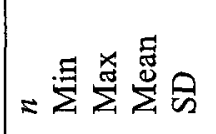 & 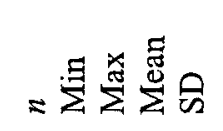 & 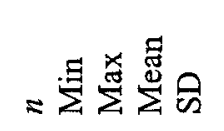 & 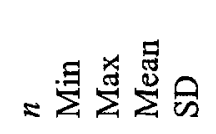 & 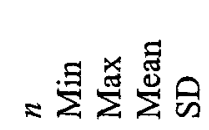 \\
\hline 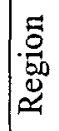 & $\stackrel{0}{\Sigma}$ & $\begin{array}{l}\dot{U} \\
z\end{array}$ & $\sum_{Z}^{E}$ & $\stackrel{\varrho}{\mathcal{O}_{2}}$ & 导 \\
\hline
\end{tabular}




\begin{tabular}{|c|c|c|c|c|c|}
\hline 紊 & 只 & oon & $\underset{n}{\infty}$ on $\stackrel{\infty}{\infty} \underset{-}{\infty}$ & oon & wo+g \\
\hline$\infty$ & $\because \begin{array}{llll}0 & 0 & \infty & 9 \\
0 & & 8 \\
0\end{array}$ & $\because 0 \backsim \sigma \frac{7}{0}$ & 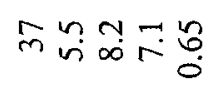 & 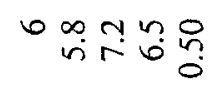 & 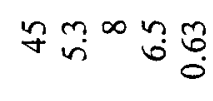 \\
\hline$\vec{\infty}$ & $2 \stackrel{\infty}{\infty}$ in & 을 min & m & 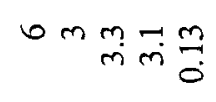 & 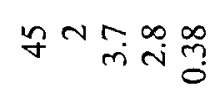 \\
\hline 泾 & 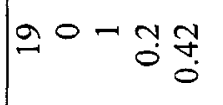 & 임ํ용 & m 0 గ & 00甘垈 & fon \\
\hline$\infty$ & $20 \sim 60$ & 으 $\mathrm{N} \underset{0}{0} \stackrel{m}{0}$ & nom & ०० $0-\frac{0}{7}$ & nom \\
\hline 5 & $20000: 8$ & 0000.8 & no- $\overrightarrow{0}$ & $000 \%: \frac{8}{0}$ & ' \\
\hline 国 & a & $\stackrel{0}{0}+\infty \stackrel{\infty}{\circ}=$ & 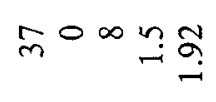 & $0-\infty \underset{i}{0} \underset{i}{\infty}$ & $\underset{\sigma}{\sigma}=\stackrel{\infty}{i} \stackrel{\infty}{\sim}$ \\
\hline$\stackrel{\omega}{\oplus}$ & 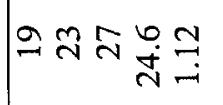 & 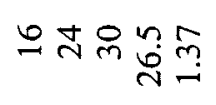 & ñ 유 只 & ๑ & 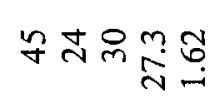 \\
\hline$\underbrace{\infty}_{\infty}$ & 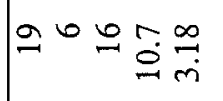 & $\stackrel{\circ}{\sim} \stackrel{\infty}{=} \underset{\sim}{=}$ & $\hat{m} \circ g \stackrel{0}{=} \underset{m}{m}$ & or $\begin{array}{r}0 \\
= \\
\end{array}$ & $\dot{q}+ニ \Xi \bar{m}$ \\
\hline$\sum_{\infty}^{\infty}$ & $=\vec{\sim} \stackrel{\sim}{\sim} \underset{\sim}{\stackrel{\sim}{-}}$ & 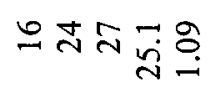 & 도유요 & 료 & 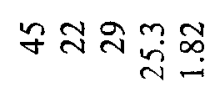 \\
\hline $\int_{\infty}^{\infty}$ & 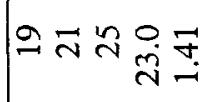 & 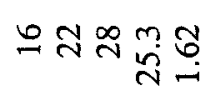 & 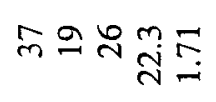 & 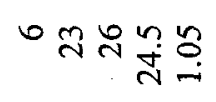 & 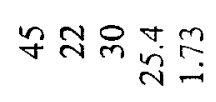 \\
\hline 莬 & 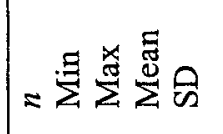 & 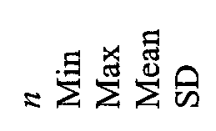 & 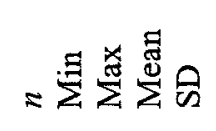 & 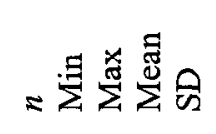 & 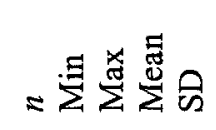 \\
\hline . & $\sum_{z}^{2}$ & 忌 & 文 & 范 & 光 \\
\hline
\end{tabular}




\begin{tabular}{|c|c|c|c|c|c|}
\hline$\sum_{2}^{\infty}$ & |fon in & 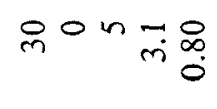 & $0 m+\underset{m}{m} \stackrel{\vec{f}}{0}$ & 䑻 N n & son $\sim$ \\
\hline$m$ & 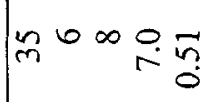 & $\stackrel{\infty}{\sim} \underset{n}{n} \mathbb{i} \overrightarrow{0}$ & 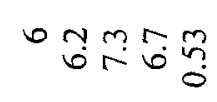 & gin? & 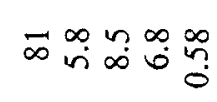 \\
\hline$\vec{n}$ & 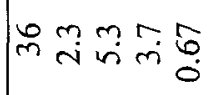 & ले & 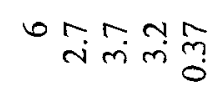 & 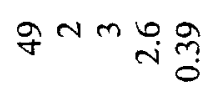 & 舫 \\
\hline 0 & mo n & 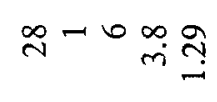 & not & 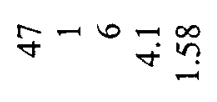 & $\hat{\infty}^{\circ} \operatorname{in} \stackrel{\infty}{0} \tilde{n}$ \\
\hline$\infty$ & lin & 闩 & b O N ? & F० NO O & চ \\
\hline 9 & 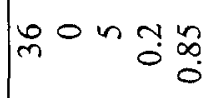 & 응- & $000 \%: 8$ & 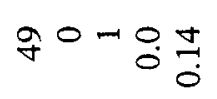 & $\hat{\infty}^{\circ}-0.0$ \\
\hline 9 & mo & oroing & oo-n & 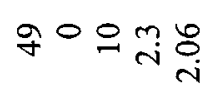 & 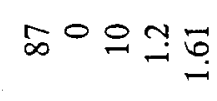 \\
\hline 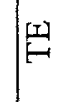 & m స̃ & 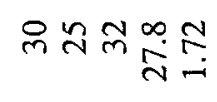 & 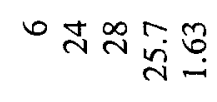 & 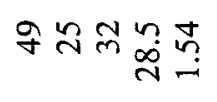 & 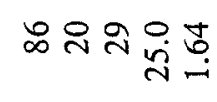 \\
\hline 己ि & $m \circ g=\underline{m}$ & 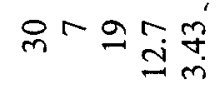 & 으유욜 & 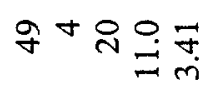 & $\vec{D}^{0} \vec{\sim} \stackrel{m}{\underline{I}} \frac{a}{m}$ \\
\hline$\sum_{\infty}^{\infty}$ & 윰유 & 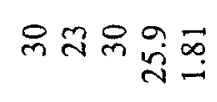 & • & gి & ๖つล \\
\hline $\int_{-19}^{\infty}$ & 유유 울 & 쥬 & • 유ํํํำ & 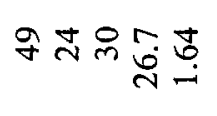 & $\stackrel{\infty}{\sim} \approx \underset{\sim}{\sim} \stackrel{\leftrightarrow}{\bullet}$ \\
\hline 语 & 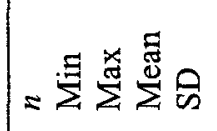 & 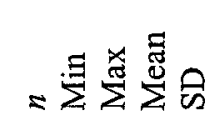 & 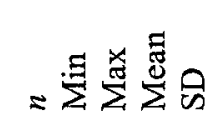 & 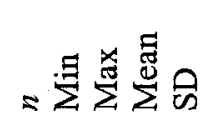 & 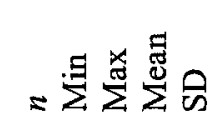 \\
\hline : & 空 & $\mathscr{U}$ & $\stackrel{\circlearrowright}{Z}$ & $\stackrel{x}{\stackrel{x}{E}}$ & $\sum_{s}^{S}$ \\
\hline
\end{tabular}




\begin{tabular}{|c|c|c|}
\hline$\frac{3}{2}$ & 20 o n $\underset{m}{\forall}$ & $\vec{N} \circ \operatorname{mm}$ \\
\hline$\tilde{m}$ & 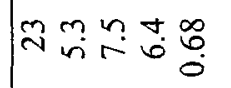 & $\vec{\sim} 0 \infty \underset{0}{\infty} \tilde{0}$ \\
\hline$\vec{m}$ & $\ddot{v} \sim m \underset{m}{m} \stackrel{m}{\sim}$ & $\vec{\sim} \sim \ddot{\forall} \underset{\sim}{\sim} \stackrel{0}{0}$ \\
\hline 0 & 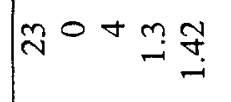 & $\vec{\sim} 0+\stackrel{\sim}{\rightarrow}$ \\
\hline$p$ & $\tilde{N}_{0} 0-\overrightarrow{0} \tilde{0}$ & $\vec{\sim} \circ m \mathscr{\sigma}_{0}^{0}$ \\
\hline 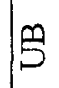 & moO $0: 8$ & $\vec{N}^{\circ-0} 0 \stackrel{0}{0}$ \\
\hline$\nexists$ & mo 0 in & $\bar{\sim}^{\circ} \circ m g$ \\
\hline $\mid \stackrel{t r \mid}{\theta}$ & స̃ & 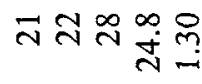 \\
\hline$\underbrace{m}_{m}$ & $\mathbb{N} r \stackrel{\sim}{m} \underset{m}{m}$ & $\bar{\sim}^{0} \forall \dot{0} \stackrel{+\infty}{i}$ \\
\hline$\sum_{2}^{\infty}$ & ลีลลัญ & $\vec{\sim} \bar{\sim} \underset{\sim}{\sim} \stackrel{8}{-}$ \\
\hline$\sum_{1}^{\infty}$ & 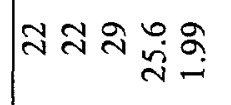 & 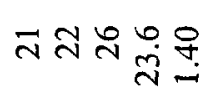 \\
\hline 量 & 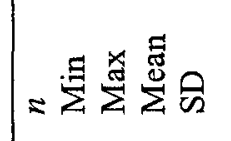 & 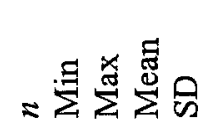 \\
\hline . & 3 & 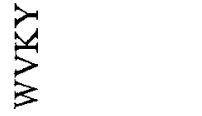 \\
\hline
\end{tabular}




\section{APPENDIX V}

\section{UNIVARIATE STATISTICS OF MERISTIC DATA FOR FEMALES}

Mean values, descriptive statistics, and ANOVAs of 24 meristic characters in 32 regions for females. Character abbreviations are defined in Appendix II, and regional abbreviations are defined in Appendix III. For each region, the following data are reported for each character: the $F$ ratio $(F)$, level of significance $(P)$, sample size for that character $(n)$, minimum value (Min), maximum value (Max), mean value (mean), and standard deviation (SD). VA indicates that nonnormality and/or heterogeneous variances prevented testing that character in ANOVA.

\begin{tabular}{|c|c|c|c|c|c|c|c|c|c|c|c|c|c|c|}
\hline Region & $\begin{array}{l}\text { Statistic } \\
F \\
P\end{array}$ & $\begin{array}{r}\text { ADS } \\
9.95 \\
0.0001\end{array}$ & $\begin{array}{r}\text { MDS } \\
\text { VA } \\
\text { VA }\end{array}$ & $\begin{array}{r}\text { PDS } \\
4.10 \\
0.0001\end{array}$ & $\begin{array}{r}\text { VS } \\
10.49 \\
0.0001\end{array}$ & $\begin{array}{r}\text { CS } \\
8.99 \\
0.0001\end{array}$ & $\begin{array}{r}\text { DCS } \\
\text { VA } \\
\text { VA }\end{array}$ & $\begin{array}{r}\text { LSL } \\
4.52 \\
0.0001\end{array}$ & $\begin{array}{r}\text { RSL } \\
4.12 \\
0.0001\end{array}$ & $\begin{array}{r}\text { LIL } \\
6.08 \\
0.0001\end{array}$ & $\begin{array}{r}\text { RIL } \\
5.18 \\
0.0001\end{array}$ & $\begin{array}{r}\text { DMB } \\
\text { VA } \\
\text { VA }\end{array}$ & $\begin{array}{r}\text { LDB } \\
16.78 \\
0.0001\end{array}$ & $\begin{array}{r}\text { RDB } \\
17.36 \\
0.0001\end{array}$ \\
\hline \multirow[t]{5}{*}{ ALMI } & $n$ & 35 & 42 & 42 & 40 & 42 & 42 & 37 & 36 & 37 & 35 & 42 & 42 & 42 \\
\hline & Min & 25 & 23 & 17 & 163 & 19 & 1 & 13 & 12 & 14 & 14 & 20 & 21 & 22 \\
\hline & $\operatorname{Max}$ & 32 & 27 & 21 & 175 & 24 & 5 & 16 & 17 & 17 & 18 & 29 & 29 & 31 \\
\hline & Mean & 28.2 & 25.0 & 19.2 & 168.9 & 21.5 & 2.0 & 14.4 & 14.3 & 15.6 & 15.7 & 25.4 & 26.3 & 26.4 \\
\hline & SD & 1.61 & 0.78 & 0.63 & 2.64 & 1.11 & 1.08 & 0.83 & 1.01 & 0.86 & 1.02 & 1.74 & 1.84 & 1.99 \\
\hline \multirow[t]{5}{*}{ APPS } & $n$ & 3 & 4 & 4 & 4 & 4 & 4 & 4 & 4 & 4 & 4 & 4 & 4 & 4 \\
\hline & Min & 25 & 23 & 18 & 164 & 18 & 1 & 13 & 13 & 14 & 15 & 24 & 25 & 24 \\
\hline & $\operatorname{Max}$ & 27 & 25 & 19 & 170 & 23 & 3 & 16 & 15 & 17 & 16 & 26 & 25 & 27 \\
\hline & Mean & 26.3 & 23.8 & 18.8 & 167.8 & 20.0 & 2.0 & 14.0 & 13.8 & 15.5 & 15.5 & 24.8 & 25.0 & 25.5 \\
\hline & SD & 1.15 & 0.96 & 0.50 & 2.63 & 2.16 & 0.82 & 1.41 & 0.96 & 1.29 & 0.58 & 0.96 & 0.00 & 1.29 \\
\hline \multirow[t]{5}{*}{ AR } & $n$ & 13 & 13 & 13 & 13 & 12 & 12 & 11 & 12 & 12 & 13 & 13 & 13 & 13 \\
\hline & Min & 26 & 23 & 19 & 167 & 21 & 1 & 13 & 13 & 14 & 14 & 22 & 23 & 22 \\
\hline & Max & 30 & 25 & 21 & 175 & 24 & 5 & 15 & 15 & 17 & 16 & 26 & 28 & 29 \\
\hline & Mean & 28.2 & 24.5 & 19.4 & 170.5 & 22.0 & 2.1 & 14.4 & 14.2 & 15.4 & 15.2 & 24.1 & 25.6 & 25.5 \\
\hline & SD & 1.24 & 0.78 & 0.77 & 2.54 & 0.95 & 1.51 & 0.81 & 0.83 & 1.00 & 0.80 & 1.26 & 1.39 & 1.94 \\
\hline
\end{tabular}




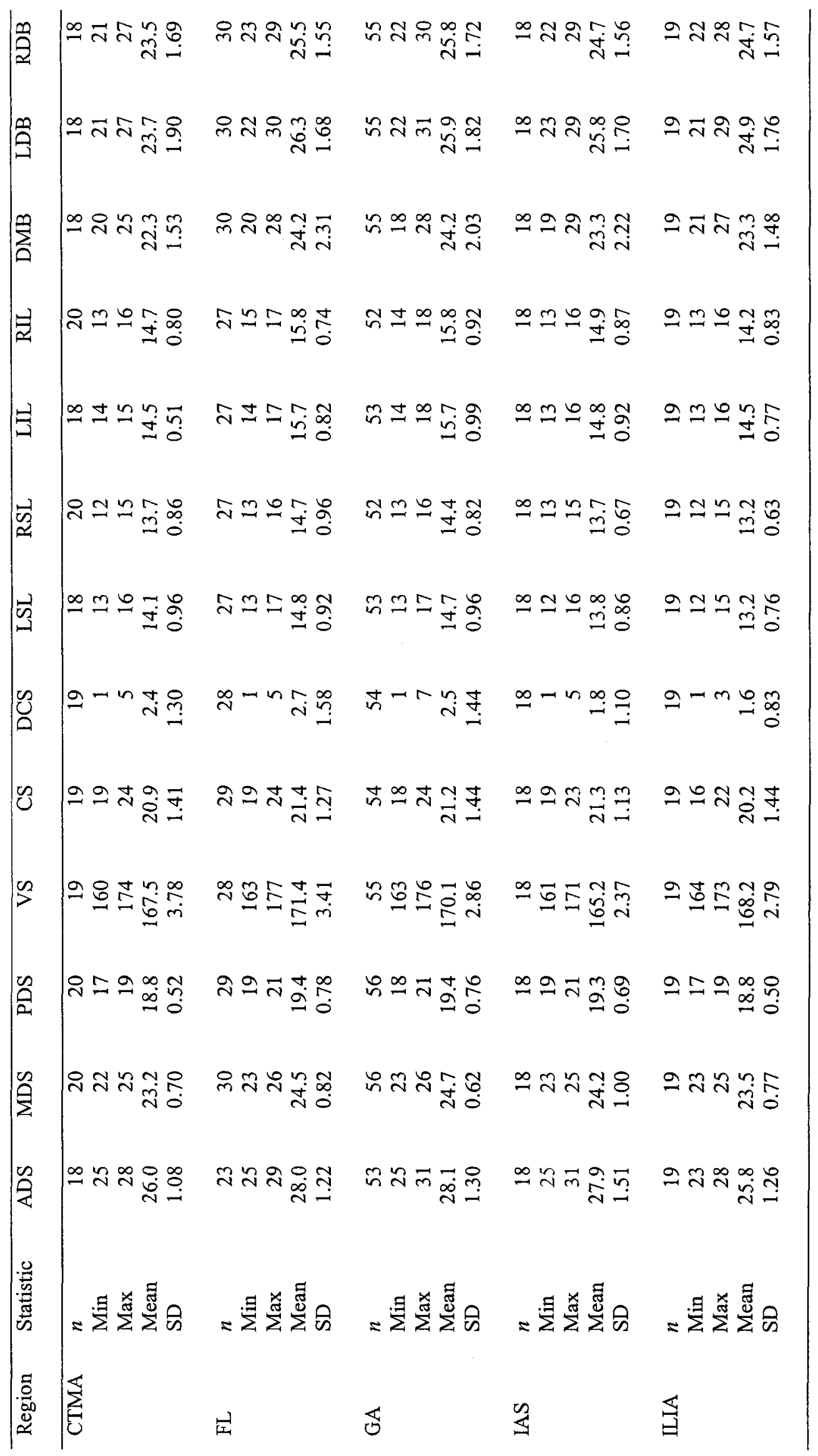




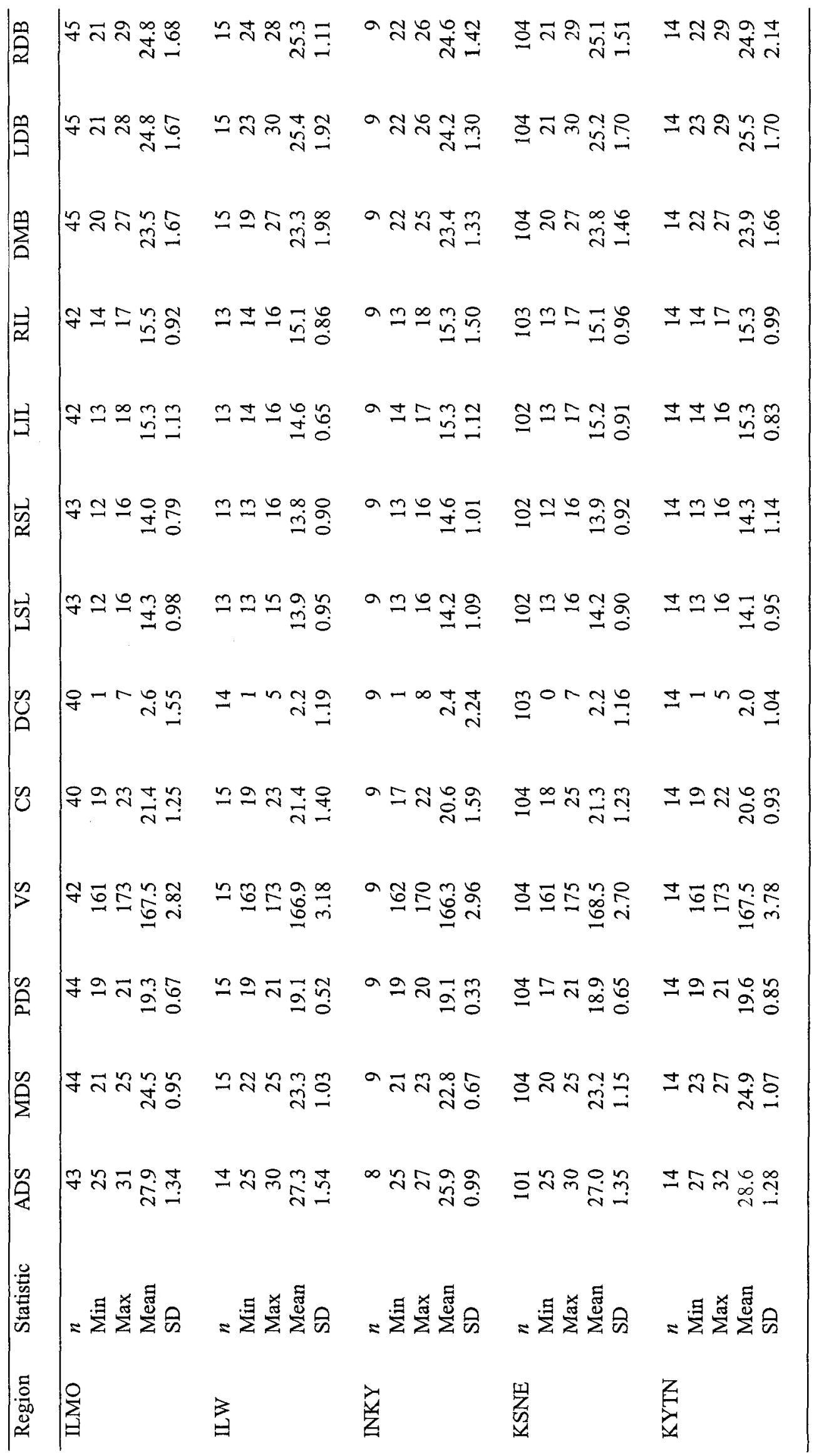




\begin{tabular}{|c|c|c|c|c|c|}
\hline$\frac{m}{\partial}$ & m유유 & 조ำ & 闹유 $\vec{n} \cong$ & 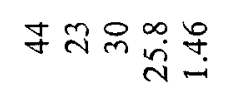 & 유유 꿀 \\
\hline ma & 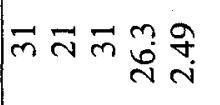 & 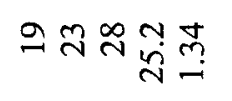 & 放空点 & サণ่ & 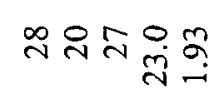 \\
\hline$\sum_{A}^{\theta}$ & $\vec{m} \pm 2 \stackrel{0}{\dot{j}} \frac{0}{m}$ & 윽유뮤뮤 & 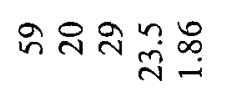 & サ̃ స̃ 导 & 요유 \\
\hline$\nexists$ & 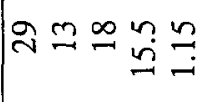 & 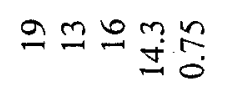 & 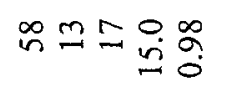 & অさニ & స士⿻一㇉丶 \\
\hline$\exists$ & 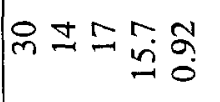 & 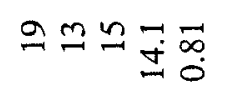 & 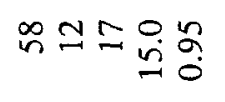 & 욤요 & 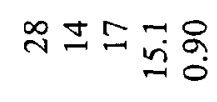 \\
\hline $\overrightarrow{\mid \overrightarrow{2}}$ & 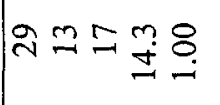 & 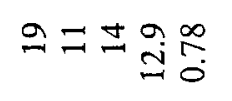 & 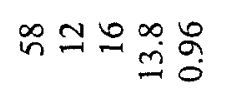 & 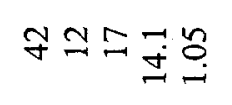 & 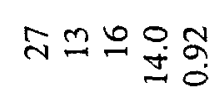 \\
\hline$\sqrt[3]{3}$ & 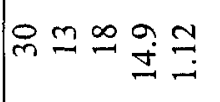 & 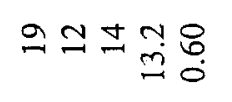 & 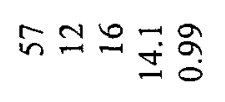 & 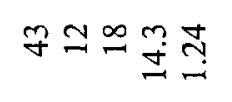 & 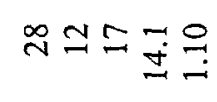 \\
\hline $\begin{array}{l}\mathscr{y} \\
\tilde{y}\end{array}$ & बิ० & $2-\nabla \stackrel{a}{-} \frac{\infty}{0}$ & $\ddot{n}+\infty \stackrel{n}{n}$ & $\exists-r \underset{i}{n} \stackrel{0}{n}$ & $\stackrel{\infty}{\sim}-n \stackrel{n}{\dddot{N}}$ \\
\hline $\mathcal{O}$ & m & 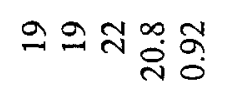 & 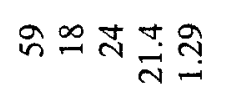 & 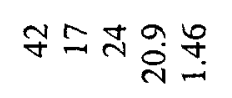 & $\stackrel{\infty}{\infty} \underset{\sim}{\sim} \stackrel{m}{\stackrel{n}{n}} \stackrel{\infty}{m}$ \\
\hline$p$ & 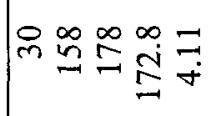 & 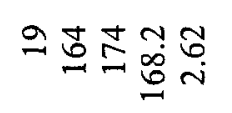 & 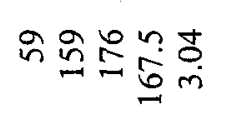 & m & 赵会点迨总 \\
\hline$\stackrel{2}{a}$ & $\bar{m} \stackrel{\infty}{-} \underset{0}{0}$ & $\stackrel{\infty}{=}$ 유욤 $\underset{\sim}{\stackrel{f}{0}}$ & ถัง & $\dot{F}=\vec{\sim} \vec{a} \dot{\sigma}$ & $\stackrel{\infty}{=}=9 \underset{2}{a}$ \\
\hline$\sum_{i}^{\infty}$ & mָন & $\stackrel{\infty}{\sim} \underset{\sim}{\sim} \tilde{N}=$ & & 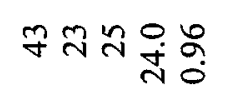 & $\stackrel{\sim}{\sim} \approx \underset{\sim}{\sim}$ \\
\hline 2 & 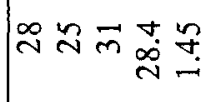 & 2 ָ̊ి & 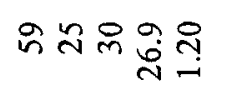 & 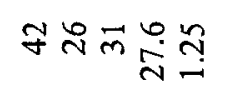 & 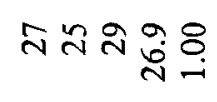 \\
\hline 总 & 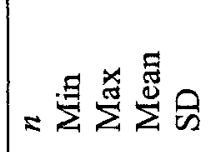 & 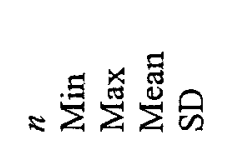 & 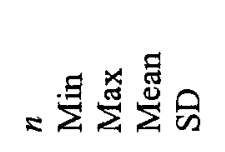 & 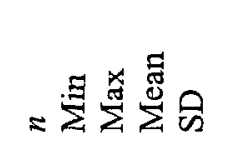 & 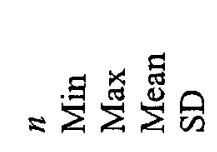 \\
\hline 壳 & $\sum_{1}$ & 胥 & $\stackrel{\circ}{\Sigma}$ & $\begin{array}{l}0 \\
0 \\
z\end{array}$ & $\sum_{Z}^{E}$ \\
\hline
\end{tabular}




\begin{tabular}{|c|c|c|c|c|c|}
\hline 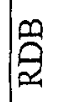 & 주료 & のㅊำ & 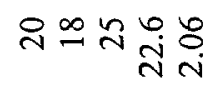 & 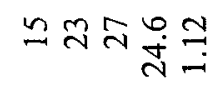 & 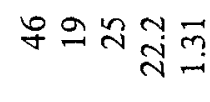 \\
\hline$\underline{m}$ & 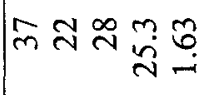 & 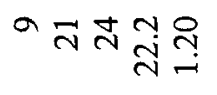 & 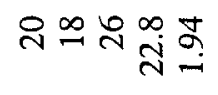 & 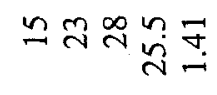 & 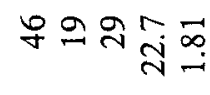 \\
\hline$\sum_{0}^{m}$ & 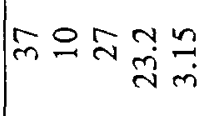 & 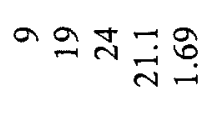 & 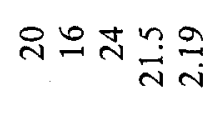 & 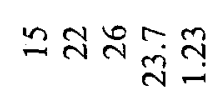 & 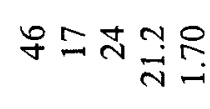 \\
\hline$\theta$ & $m \Xi \approx \underset{n}{m} \stackrel{m}{m}$ & 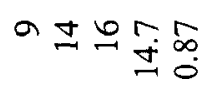 & 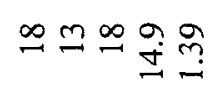 & 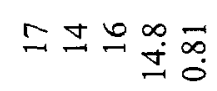 & 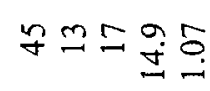 \\
\hline$\exists$ & 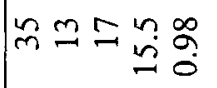 & amニ梁 & 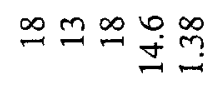 & 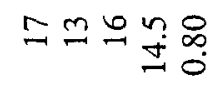 & オのニ导合 \\
\hline$\frac{1}{2}$ & $m \simeq 0 a \hat{g}$ & $a \cong n \underset{9}{0}$ & 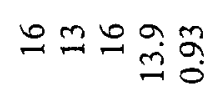 & 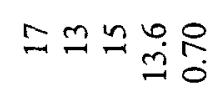 & 莳윰요 \\
\hline चे & 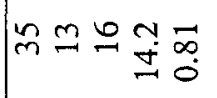 & am正虽 & 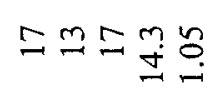 & ニッュت゚ & 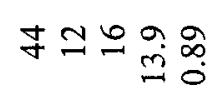 \\
\hline 里 & $\hat{m}-0 \vec{i} \stackrel{m}{m}$ & $\infty-\nabla \stackrel{\infty}{\underset{i}{0}}$ & 유- & $=-\infty \stackrel{\infty}{i} \stackrel{n}{\sigma}$ & 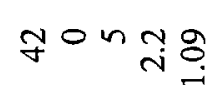 \\
\hline U & $m \stackrel{\infty}{=} \underset{i}{\stackrel{i}{i}}$ & 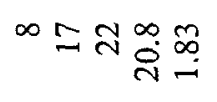 & 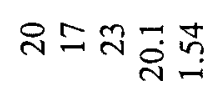 & 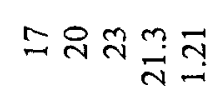 & 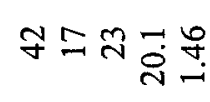 \\
\hline 3 & $\tilde{m}$ & 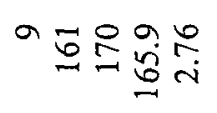 & 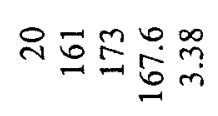 & 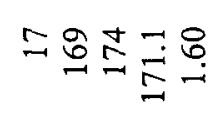 & 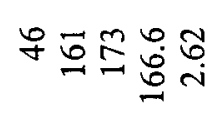 \\
\hline â & $\tilde{m}=\vec{\sim} \vec{a} \stackrel{n}{0}$ & 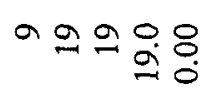 & 므유 & $=\stackrel{\infty}{=} \underset{\infty}{\infty} \stackrel{m}{0}$ & 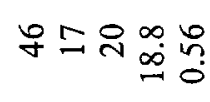 \\
\hline$\overbrace{i}^{\infty}$ & $\bar{m} \vec{\sim} \approx \underset{\dot{j}}{\dot{+}}$ & 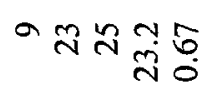 & ㅇনฺ & $=\bar{\sim} \approx \stackrel{\mathrm{N}}{\mathrm{O}}$ & 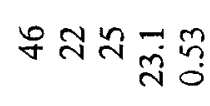 \\
\hline$\frac{\infty}{9}$ & 足只 & 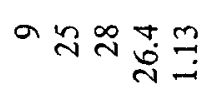 & 옥요윰 & $=\approx$ సి & 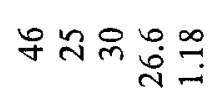 \\
\hline 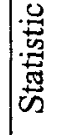 & 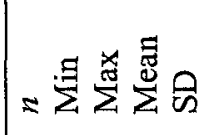 & 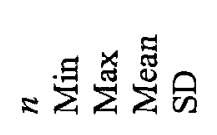 & 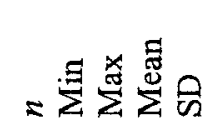 & 二見希焉怘 & 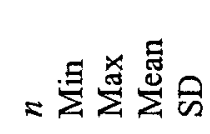 \\
\hline 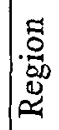 & 会 & 㝕 & 至 & 㐘 & 岂 \\
\hline
\end{tabular}




\begin{tabular}{|c|c|c|c|c|c|}
\hline 齐 & 约 & 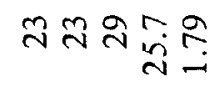 & ตำ & 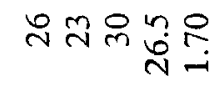 & 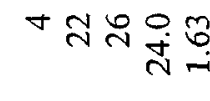 \\
\hline$\stackrel{m}{\stackrel{m}{\Omega}}$ & 哧苛 & 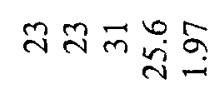 & 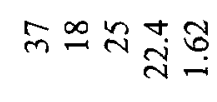 & 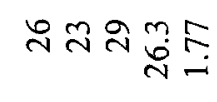 & + ㅁำ \\
\hline$\sum_{i}^{m}$ & nचี & ఇ유 六 & ñ & 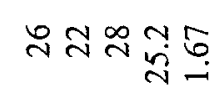 & $\forall \vec{\sim} \underset{\mathrm{N}}{ }$ \\
\hline 国 & $n \pm 00$ & $\approx \cong \approx \tilde{a}$ & $\hat{m}=\because \underset{\dot{\Xi}}{0} 0$ & 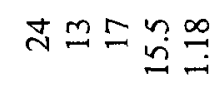 & 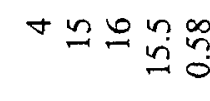 \\
\hline 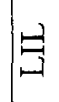 & nエ & 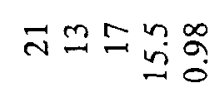 & 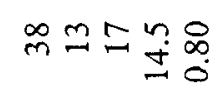 & 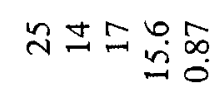 & $\forall \pm 00 \stackrel{0}{0} \underset{0}{\infty}$ \\
\hline 崖 & 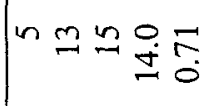 & ํํำ & $\begin{array}{l}\infty \\
m \simeq 0 \\
\dot{m}\end{array}$ & 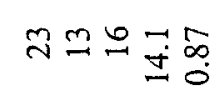 & 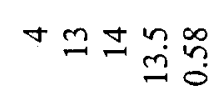 \\
\hline 岕 & 的士兄怘志 & 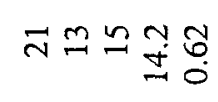 & $\tilde{n} \cong \stackrel{\infty}{=} \underset{\dot{J}}{0}$ & 芯 & ナ士幺羿号 \\
\hline $\begin{array}{l}\infty \\
0 \\
0\end{array}$ & $n-\forall \underset{1}{n}$ & 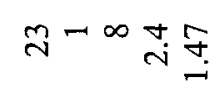 & $\stackrel{\infty}{m}-6 \stackrel{i}{\mathcal{F}}$ & $\tilde{i}-n \stackrel{i}{i}$ & $+-N \dddot{n}$ \\
\hline$\tilde{Z}$ & 咛 & 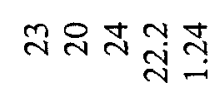 & 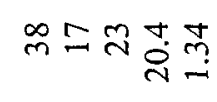 & 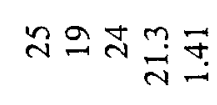 & ナશন율 \\
\hline$\rho$ & 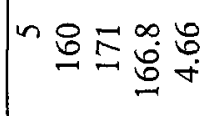 & 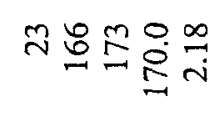 & 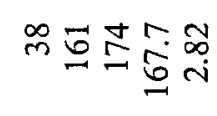 & 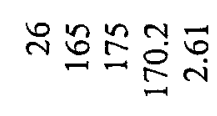 & 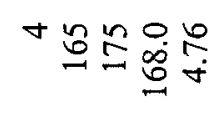 \\
\hline$\stackrel{2}{2}$ & in 290.8 & & $\begin{array}{l}\infty \\
m\end{array}$ & 总穴泀。 & ナ9. \\
\hline$\overbrace{2}^{\infty}$ & n & 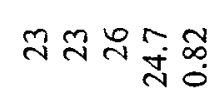 & $\stackrel{\infty}{m} \vec{\sim} \approx \underset{\tilde{N}}{\tilde{N}}$ & 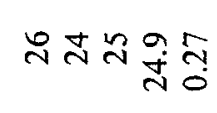 & 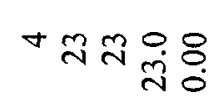 \\
\hline 惩 & 约 & 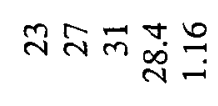 & 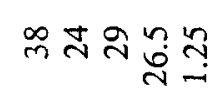 & 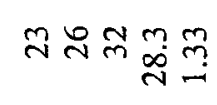 & অ \\
\hline 惫 & $=$ 吉总寻怘会 & 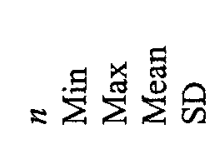 & 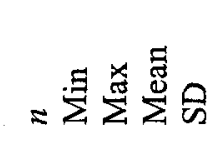 & 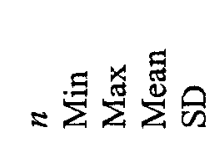 & 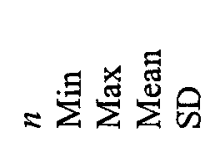 \\
\hline 离 & 些 & $\ddot{b}$ & 跑 & U & Z \\
\hline
\end{tabular}




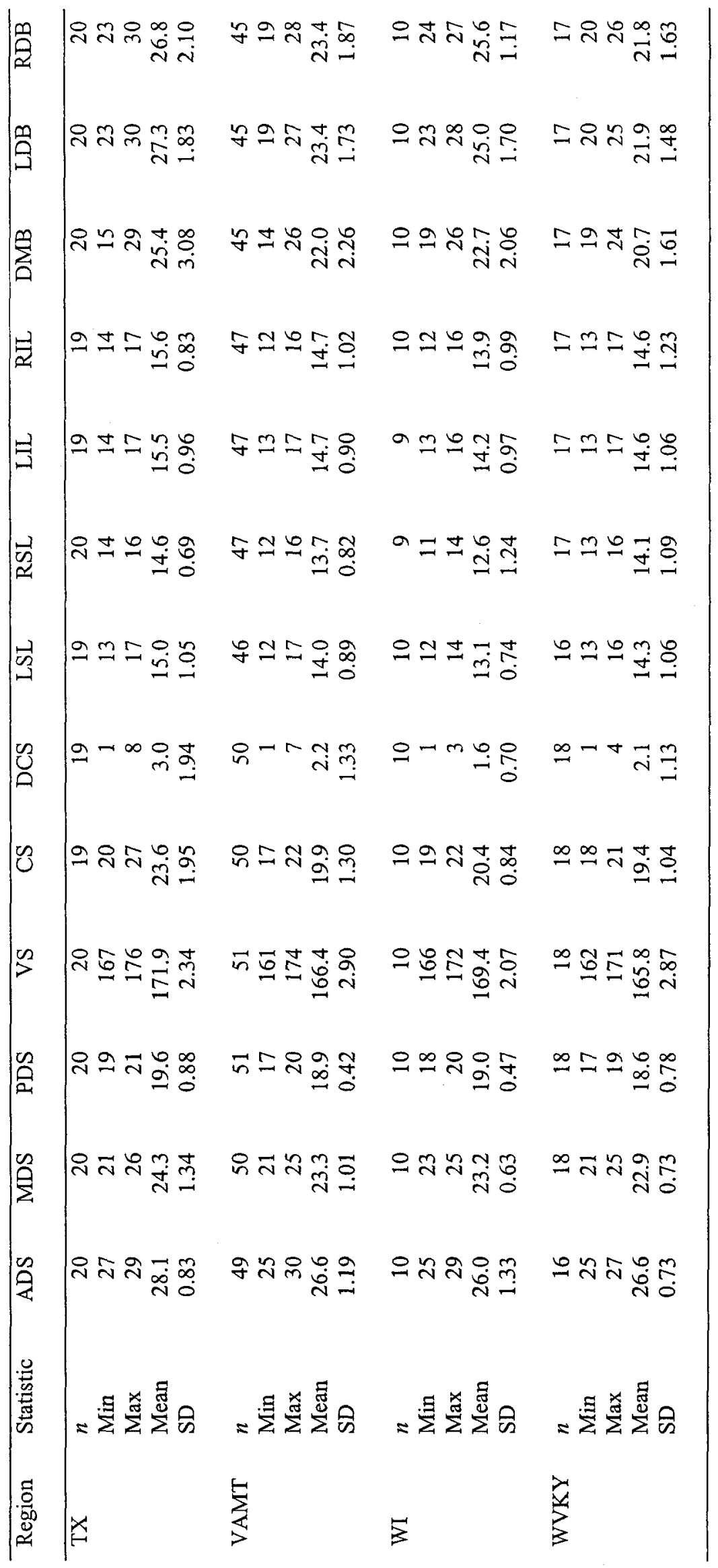




\begin{tabular}{|c|c|c|c|c|c|}
\hline 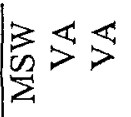 & mo & $\nabla m \nabla \sim n$ & $\simeq m+\underset{m}{m} \stackrel{m}{0}$ & Oong & $\stackrel{\infty}{m}+\vec{m} \overrightarrow{\tilde{n}}$ \\
\hline 象是豆 & 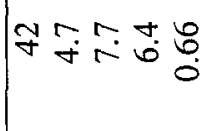 & $+N \hat{6}: 0: 0$ & $=0 r i \frac{\infty}{r}$ & $m \underset{\sigma}{m} \sim$ & 只 \\
\hline 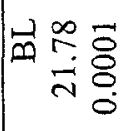 & 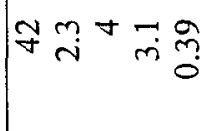 & 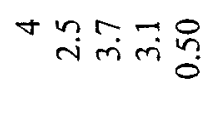 & 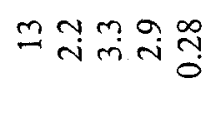 & 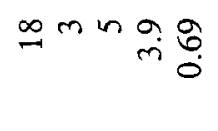 & 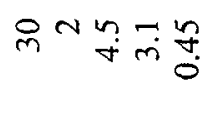 \\
\hline$\bigoplus_{\forall} \lesssim$ & fon & $+0+\underset{i}{0} \underset{i}{8}$ & NO & $20+\tilde{0} \stackrel{\infty}{?}$ & ते \\
\hline$\stackrel{\lambda}{>}$ & Fom & to-rn & mo-ñ & $\stackrel{\infty}{=}$ O & 유유 중 \\
\hline$b \lesssim$ & 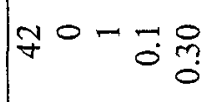 & ナo-m? & mo0 $: 8$ & $20-\overrightarrow{0} \frac{\pi}{0}$ & 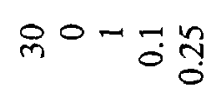 \\
\hline$m \leq$ & Fon $\underset{I}{-} \vec{I}$ & tom 악 & momg & $\stackrel{\infty}{0} 00=\overline{-}$ & pr-om \\
\hline F & F & 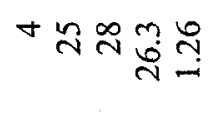 & 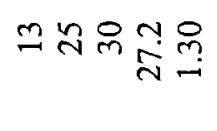 & 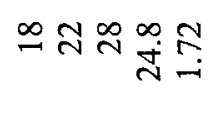 & 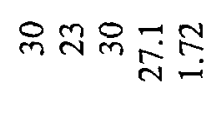 \\
\hline 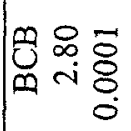 & $\vec{\sigma} 0=\underset{z}{\vec{i}}$ & ナa욜 & $\bigcap^{\infty} 0 \underset{0}{0} \overrightarrow{0}$ & $\stackrel{\infty}{\Rightarrow}=\stackrel{0}{=} \stackrel{a}{=}$ & 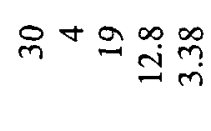 \\
\hline 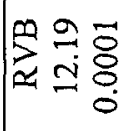 & 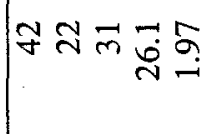 & 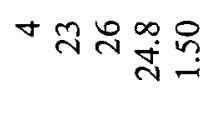 & 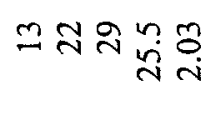 & 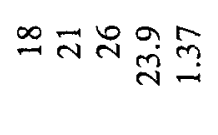 & 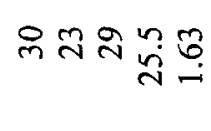 \\
\hline 皇 & 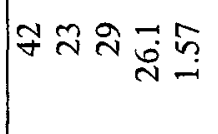 & 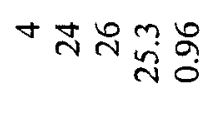 & 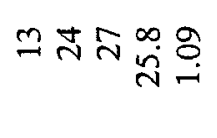 & $\stackrel{\infty}{\sim}$ 유 导 & 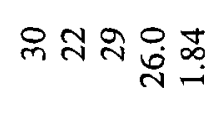 \\
\hline 总 & 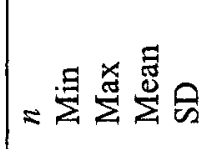 & 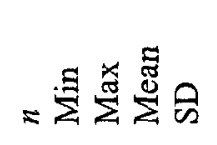 & 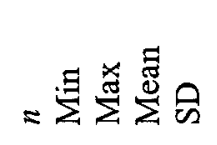 & 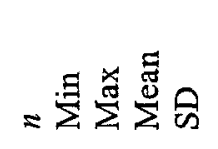 & 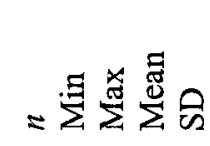 \\
\hline $\mid \begin{array}{l}0 \\
5 \\
0 \\
2 \\
2\end{array}$ & 灵 & 先 & $\frac{2}{4}$ & $\sum_{j}^{\bigsqcup}$ & 崩 \\
\hline
\end{tabular}




\begin{tabular}{|c|c|c|c|c|c|}
\hline$\sum_{\substack{n \\
\Sigma}}^{3}$ & ino in $\hat{i} \frac{\hat{\sigma}}{0}$ & $\stackrel{\infty}{\rightarrow}$ o in & 2 ○ in & mon & $\stackrel{\sim}{\sim} \circ \underset{\sim}{\nabla} \stackrel{\infty}{\leftrightarrows}$ \\
\hline$\infty$ & Hin mio & 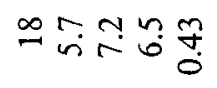 & 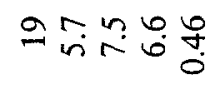 & খণ் & 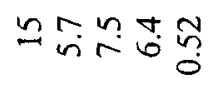 \\
\hline$\vec{\omega}$ & $\ddot{n} \tilde{i} \underset{\dot{r}}{m} \stackrel{n}{0}$ & $\stackrel{\infty}{\rightarrow} \underset{i}{m} \stackrel{\infty}{i} \stackrel{i}{0}$ & 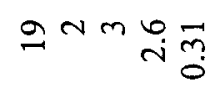 & 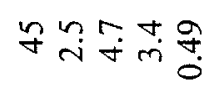 & $\approx$ Nलिं \\
\hline$\Theta_{1}^{\infty}$ & in o n & 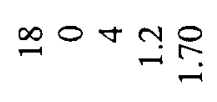 & $20 \mathrm{~m}: 0$ & Y & $\underline{\sim} 0+\underset{r}{\Phi}$ \\
\hline$m$ & 绳 0 N & $\stackrel{\infty}{=}$ ON & 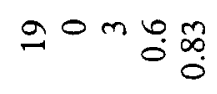 & 管om & תON过 \\
\hline s & $\ddot{n} 0-\overrightarrow{0} \underset{0}{\tilde{0}}$ & 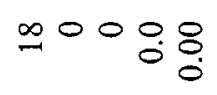 & $20-\frac{1}{0}$ ? & 为 & $\cong 00 \%$ \\
\hline 象 & nol $\ln ^{n}$ & $\stackrel{\infty}{\rightarrow} \underset{\sim}{\infty} \underset{\dot{m}}{\tilde{m}}$ & gor $\overline{\mathrm{i}} \stackrel{\text { 品 }}{ }$ & \% & nO⿻m mir \\
\hline 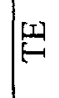 & 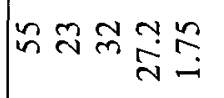 & $\stackrel{\infty}{\sim} \overrightarrow{\text { 它 }}$ & ح乞ㅇำ & 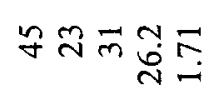 & 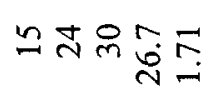 \\
\hline 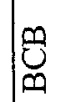 & $\ddot{n}-\vec{\sim} \underset{\sim}{\stackrel{N}{N}}$ & 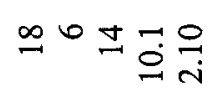 & 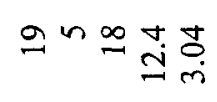 & $\mathscr{\gamma}+\curvearrowleft \underset{\sim}{\sim} \tilde{\sim}$ & $\ddot{\theta}+g \hat{\theta}$ \\
\hline$\sum_{\infty}^{\infty}$ & 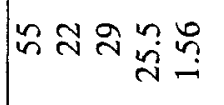 & $\stackrel{2}{=}$ 굼 & 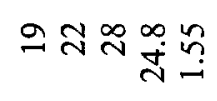 & ঋ 유 $\stackrel{+}{+}$ & 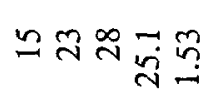 \\
\hline$\sum_{i=1}^{\infty}$ & | & $\stackrel{\infty}{=} \approx$ 굼 & શิ సి & 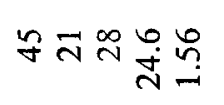 & 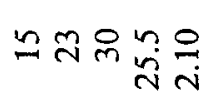 \\
\hline 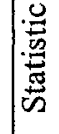 & 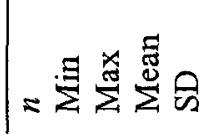 & 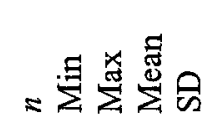 & 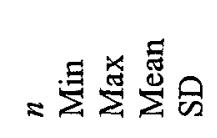 & 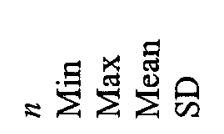 & 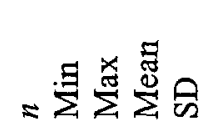 \\
\hline 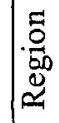 & $\mathbb{\Xi}$ & $\underset{1}{S}$ & $\underset{\Xi}{\leftrightarrows}$ & $\sum_{\exists}^{O}$ & 音 \\
\hline
\end{tabular}




\begin{tabular}{|c|c|c|c|c|c|}
\hline$\sum$ & aromo & $\underset{0}{0} \circ \ln m \underset{0}{m} \stackrel{\infty}{\infty}$ & 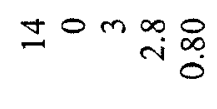 & 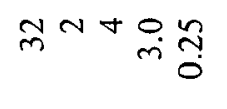 & $\stackrel{\infty}{\sim} \underset{\sim}{\sim} \stackrel{0}{\sim}$ \\
\hline 2 & av & 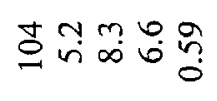 & $\pm \underset{n}{\infty} \stackrel{\infty}{\infty} \underset{0}{\infty}$ & 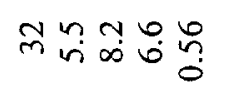 & 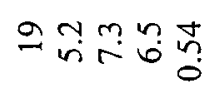 \\
\hline$\vec{m}$ & $\infty \underset{\sim}{\infty}+\underset{n}{n} \underset{0}{q}$ & 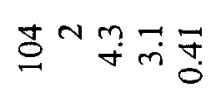 & $\pm \hat{i} \stackrel{\sim}{+} \stackrel{m}{m} \stackrel{\infty}{0}$ & 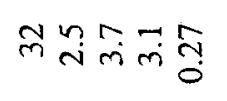 & $\therefore N m \vec{\sim}$ \\
\hline$\theta_{1}^{\infty}$ & 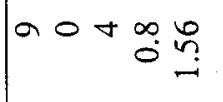 & to+g & $\pm 0+\underset{-i}{0}$ & ลิ०+थ & ๑ON \\
\hline 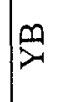 & aror & tomm & Jom $\check{0}:$ & $\vec{m} 0-\overrightarrow{0} \dot{0}$ & פON \\
\hline 9 & 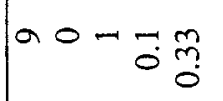 & 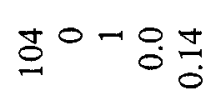 & $\pm 00 \bigcirc:$ & mo00: & $90-\breve{0}$ \\
\hline 田 & aON & 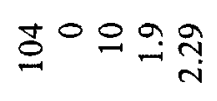 & JON & $\vec{m} \circ \simeq \vec{i} \underset{i}{n}$ & gon \\
\hline 㸃 & 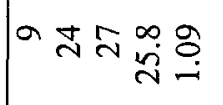 & 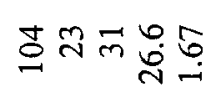 & ఫむ் & $\bar{m} \vec{\sim} \bar{m} \underset{\sim}{\sim} \underset{\sim}{\sim}$ & 휴 \\
\hline ن্ & abt合朵 & 음 & $\Xi N=\stackrel{0}{0} \stackrel{\infty}{0}$ & $\vec{m} m a g \frac{a}{m}$ & $g \circ g \stackrel{g}{\underline{m}} \stackrel{\sim}{m}$ \\
\hline$\sum_{\infty}^{\infty}$ & 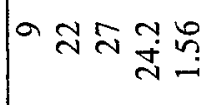 & 苍ন & 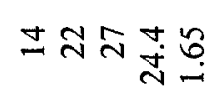 & 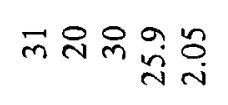 & 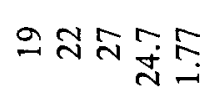 \\
\hline 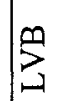 & 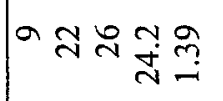 & 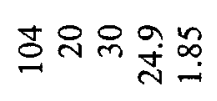 & মむন & 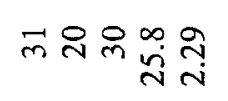 & 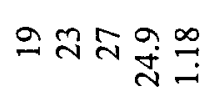 \\
\hline 焉 & 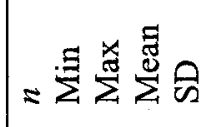 & 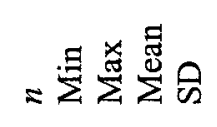 & =寻总寻芯 & 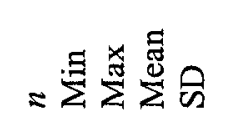 & 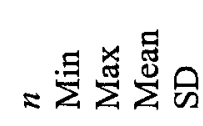 \\
\hline 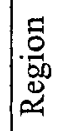 & 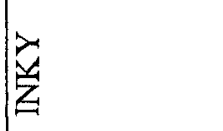 & 至 & 忩 & 岁 & z \\
\hline
\end{tabular}




\begin{tabular}{|c|c|c|c|c|c|}
\hline 窝 & 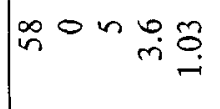 & $\operatorname{Jan} m m_{m}^{\infty}$ & $\stackrel{\infty}{\sim} \circ \cdots \underset{m}{\vec{m}}$ & mmn mi & a o in \\
\hline m & 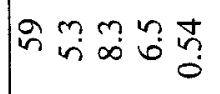 & 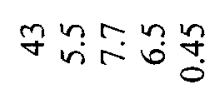 & $\approx 0+\frac{1}{0}$ & nñ⿻日禸 & n \\
\hline$\ddot{m}$ & 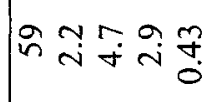 & $\overrightarrow{+} \underset{\mathrm{N}}{+\vec{m}} \overline{\mathrm{m}}$ & শ্ m & $\hat{m} \stackrel{i}{\vec{m}} \stackrel{\infty}{0}$ & 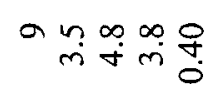 \\
\hline 0 & not I & $\operatorname{Jon} \underset{\operatorname{I}}{\operatorname{g} \sigma}$ & సิ & no+ & a 000.8 \\
\hline 2 & non & $\operatorname{Jom} 0 \frac{0}{0}$ & స̃ & ñ & a O \\
\hline 5 & in $000 \%$ & 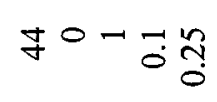 & ते $0-\overrightarrow{0} \tilde{0}$ & ñ & 9000.8 \\
\hline 呈 & $\ln ^{\circ} \circ 0 . \underset{i}{0}$ & 近尔 & స๐ํㅇㅇㅇ & 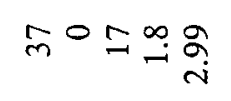 & GON: \\
\hline 照 & |ำ & J莳 & 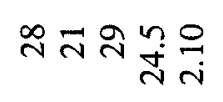 & 께유윰요 & 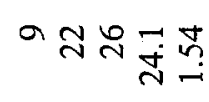 \\
\hline$\underbrace{\infty}_{\infty}$ & $\sin 0$ 응 & 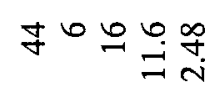 & 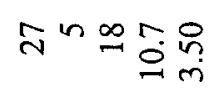 & 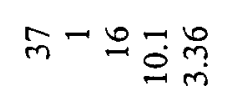 & 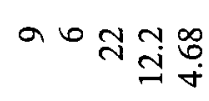 \\
\hline$\sum_{\infty}^{\infty}$ & 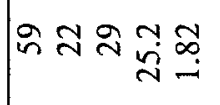 & サঐి & 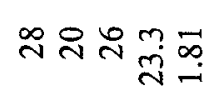 & 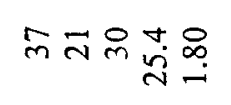 & の రి \\
\hline$\sum_{1}^{\infty}$ & 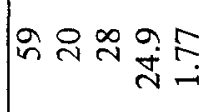 & サ & শ্ণ & 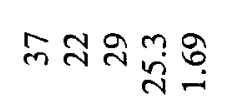 & $a \bar{\sim} \underset{\sim}{\infty} \stackrel{\infty}{g}$ \\
\hline$\frac{\pi}{4}$ & 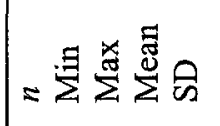 & 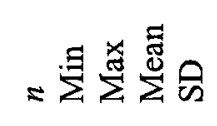 & 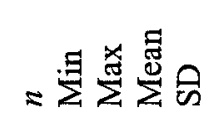 & 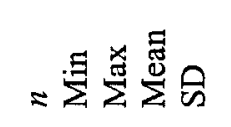 & 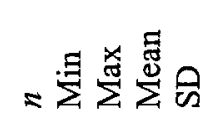 \\
\hline 范 & $\stackrel{\varrho}{\Sigma}$ & 总 & $\sum_{Z}^{H}$ & 导 & 它 \\
\hline
\end{tabular}




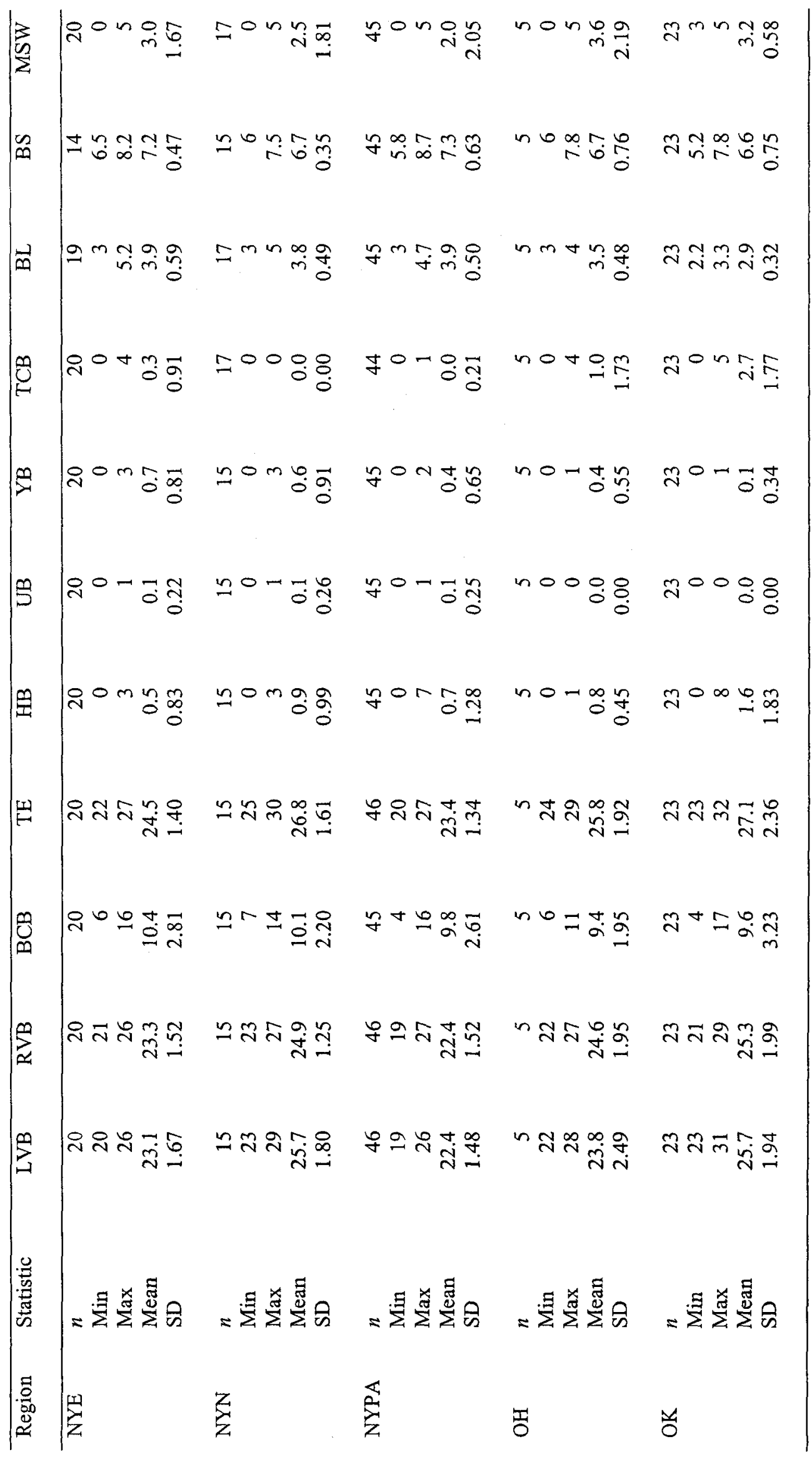




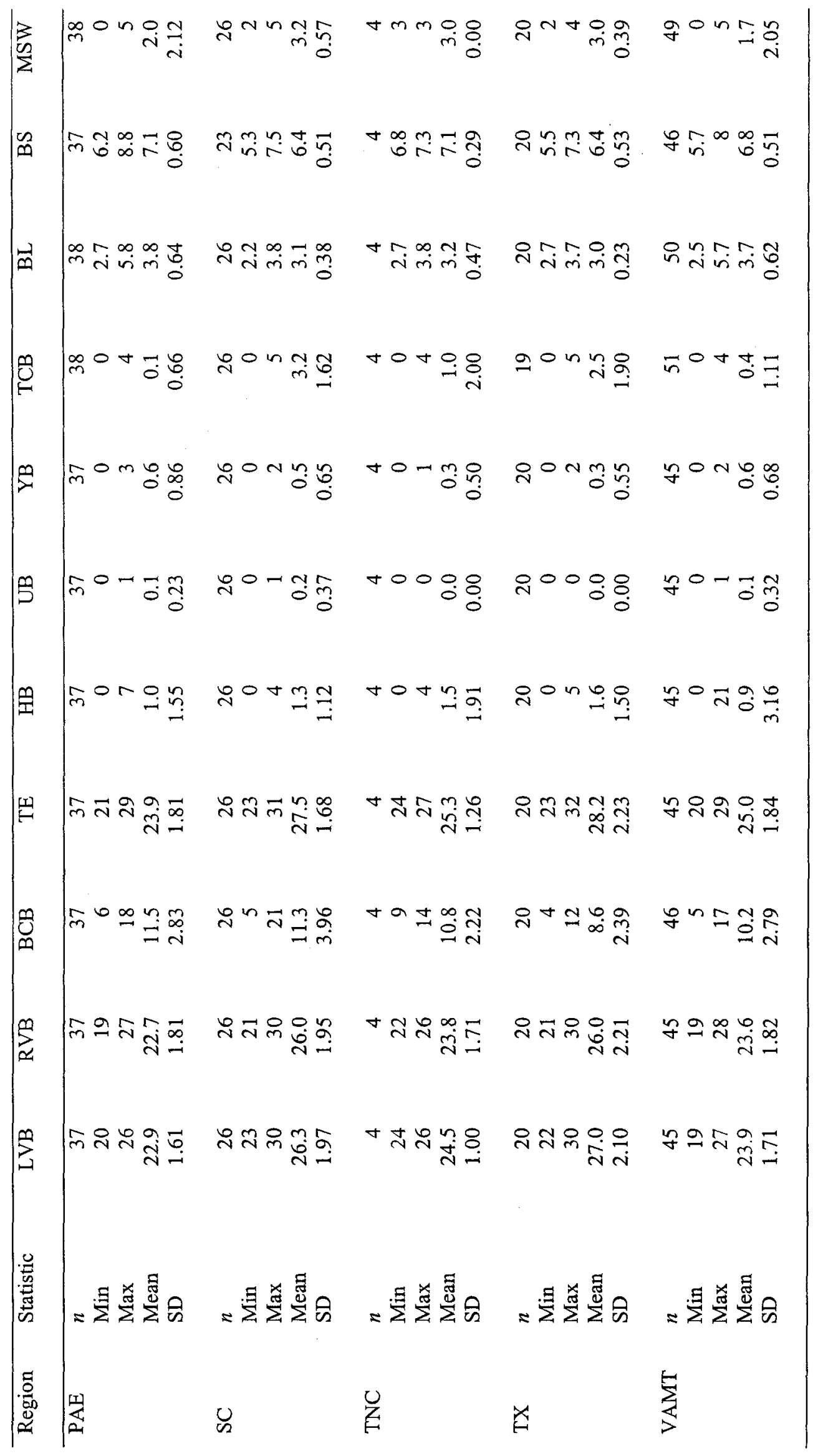




\begin{tabular}{|c|c|c|}
\hline 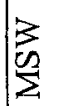 & 100+ & 임 \\
\hline $\mathscr{m}$ & 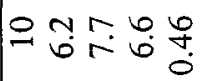 & $\infty$ \\
\hline$\vec{n}$ & O Nm & 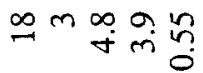 \\
\hline రृ & $\stackrel{0}{\circ} 0 \mathrm{~m} n \stackrel{\infty}{\circ}$ & $\stackrel{\infty}{\sim} 0-\overline{0} \stackrel{⿱}{\sim}$ \\
\hline$\infty$ & 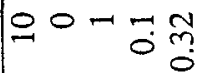 & =0-むั。 \\
\hline 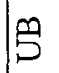 & 잉ㅇㅇㅇ.: & 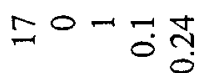 \\
\hline 里 & 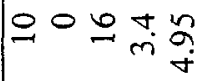 & 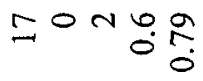 \\
\hline 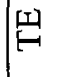 & 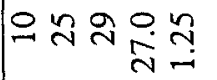 & 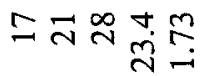 \\
\hline 论 & $O 0=\hat{\mathrm{j}} \hat{\mathrm{m}}$ & = \\
\hline$\sum_{x}^{\infty}$ & 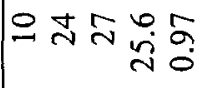 & 유ন \\
\hline$\sum^{\infty}$ & 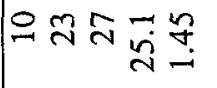 & 듀뮴ำ \\
\hline 蒡 & 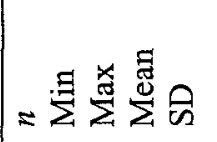 & 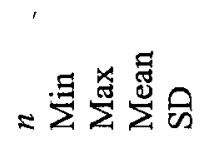 \\
\hline .0 & $\$$ & 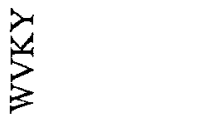 \\
\hline
\end{tabular}




\section{APPENDIX VI}

\section{UNIVARIATE STATISTICS OF MERISTIC DATA FOR COMBINED SEXES}

Mean values, descriptive statistics, and ANOVAs of 24 meristic characters in 32 regions for combined sexes. Character abbreviations are defined in Appendix II, and regional abbreviations are defined in Appendix III. For each region, the following data are reported for each character: the $F$ ratio $(F)$, level of significance $(P)$, sample size for that character $(n)$, minimum value (Min), maximum value (Max), mean value (mean), and standard deviation (SD). VA indicates that nonnormality and/or heterogeneous variances prevented testing that character in ANOVA, and NA indicates that the character was not applicable for combined sexes.

\begin{tabular}{|c|c|c|c|c|c|c|c|c|c|c|c|c|c|c|}
\hline Region & $\begin{array}{l}\text { Statistic } \\
F \\
P\end{array}$ & $\begin{array}{r}\mathrm{ADS} \\
22.25 \\
0.0001\end{array}$ & $\begin{array}{r}\text { MDS } \\
33.00 \\
0.0001\end{array}$ & $\begin{array}{r}\text { PDS } \\
\text { VA } \\
\text { VA }\end{array}$ & $\begin{array}{l}\text { VS } \\
\text { NA } \\
\text { NA }\end{array}$ & $\begin{array}{l}\text { CS } \\
\text { NA } \\
\text { NA }\end{array}$ & $\begin{array}{r}\text { DCS } \\
\text { VA } \\
\text { VA }\end{array}$ & $\begin{array}{r}\text { LSL } \\
7.50 \\
0.0001\end{array}$ & $\begin{array}{r}\text { RSL } \\
8.29 \\
0.0001\end{array}$ & $\begin{array}{r}\text { LIL } \\
13.41 \\
0.0001\end{array}$ & $\begin{array}{r}\text { RIL } \\
11.90 \\
0.0001\end{array}$ & $\begin{array}{r}\text { DMB } \\
\text { VA } \\
\text { VA }\end{array}$ & $\begin{array}{r}\text { LDB } \\
38.20 \\
0.0001\end{array}$ & $\begin{array}{r}\mathrm{RDB} \\
39.73 \\
0.0001\end{array}$ \\
\hline \multirow[t]{5}{*}{ ALMI } & $n$ & 82 & 92 & 92 & 90 & 92 & 92 & 83 & 80 & 84 & 80 & 92 & 92 & 92 \\
\hline & Min & 25 & 23 & 17 & 157 & 19 & 1 & 12 & 12 & 14 & 14 & 20 & 21 & 22 \\
\hline & Max & 33 & 27 & 21 & 175 & 29 & 7 & 16 & 17 & 18 & 18 & 30 & 32 & 32 \\
\hline & Mean & 28.3 & 25.0 & 19.2 & 166.8 & 23.9 & 2.0 & 14.3 & 13.9 & 15.8 & 15.7 & 25.7 & 26.7 & 26.8 \\
\hline & $\mathrm{SD}$ & 1.48 & 0.70 & 0.57 & 3.28 & 2.52 & 1.17 & 0.91 & 1.00 & 0.94 & 0.95 & 1.82 & 1.80 & 1.92 \\
\hline \multirow[t]{5}{*}{ APPS } & $n$ & 14 & 15 & 15 & 15 & 15 & 15 & 15 & 15 & 15 & 15 & 15 & 15 & 15 \\
\hline & Min & 25 & 23 & 18 & 159 & 18 & 1 & 13 & 13 & 14 & 15 & 12 & 23 & 22 \\
\hline & Max & 30 & 25 & 21 & 170 & 26 & 4 & 16 & 15 & 17 & 17 & 28 & 30 & 29 \\
\hline & Mean & 27.6 & 23.9 & 19.3 & 164.1 & 23.8 & 2.1 & 13.9 & 13.8 & 15.7 & 15.6 & 24.0 & 25.8 & 25.7 \\
\hline & $\mathrm{SD}$ & 1.39 & 0.96 & 0.70 & 3.18 & 2.70 & 0.88 & 0.92 & 0.68 & 0.82 & 0.63 & 3.70 & 1.66 & 1.88 \\
\hline \multirow[t]{5}{*}{$\mathrm{AR}$} & $n$ & 34 & 34 & 34 & 34 & 32 & 32 & 31 & 33 & 31 & 34 & 34 & 34 & 34 \\
\hline & Min & 26 & 23 & 18 & 161 & 21 & 1 & 13 & 12 & 14 & 14 & 19 & 21 & 22 \\
\hline & Max & 31 & 27 & 21 & 175 & 29 & 6 & 15 & 15 & 17 & 17 & 27 & 28 & 29 \\
\hline & Mean & 28.4 & 24.7 & 19.5 & 167.2 & 24.8 & 2.4 & 14.2 & 14.0 & 15.3 & 15.3 & 23.9 & 25.5 & 25.6 \\
\hline & $\mathrm{SD}$ & 1.28 & 0.83 & 0.86 & 3.80 & 2.54 & 1.62 & 0.79 & 0.88 & 0.87 & 0.98 & 1.88 & 1.78 & 1.89 \\
\hline
\end{tabular}




\begin{tabular}{|c|c|c|c|c|c|}
\hline 常 & $\forall \bar{\nabla} \underset{\sim}{0} \stackrel{n}{n}$ & 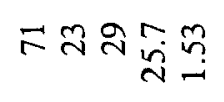 & 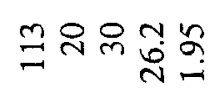 & 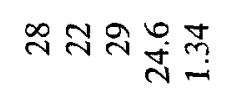 & 궈유욤 \\
\hline$\stackrel{m}{\Theta}$ & 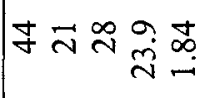 & 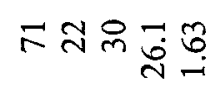 & $\exists \approx \vec{m} \underset{\mathrm{N}}{\tilde{G}}$ & 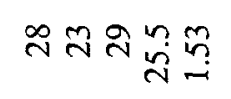 & 누규요 \\
\hline$\sum_{0}^{m}$ & 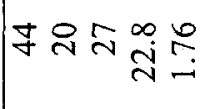 & $F \cong \stackrel{\infty}{\sim} \underset{\sim}{\infty} \underset{\sim}{\infty}$ & $\stackrel{m}{=} \stackrel{\sim}{\stackrel{\sim}{+}}$ & 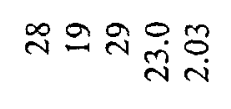 & 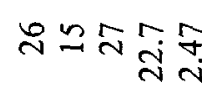 \\
\hline$\Leftrightarrow$ & 舟电守 & 过文怘 & 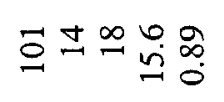 & নョニ芯す & $\mathscr{N} \cong \simeq \cong$ \\
\hline$\exists$ & F⿻ำ & $\vec{\sigma} \cong \infty \stackrel{\infty}{\sim} \stackrel{m}{0}$ & 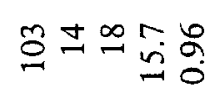 & $\stackrel{\infty}{\sim}=\stackrel{\infty}{ \pm}$ & 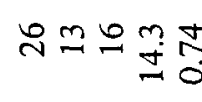 \\
\hline$\underline{\cong}$ & $\forall \approx n \underset{m}{\infty}$ & 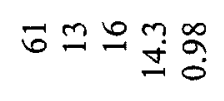 & 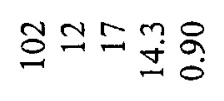 & 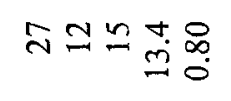 & 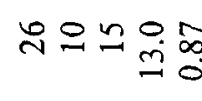 \\
\hline$\sqrt{3}$ & 죽요용 & $\mathcal{O} \cong=\underset{+}{\forall}$ & $\cong \approx \cong \underset{\Xi}{ \pm}$ & $\stackrel{\sim}{\sim} \cong \stackrel{\sim}{\sim} \underset{m}{\sim} \stackrel{n}{0}$ & 융으뭉 \\
\hline Uू & $g-\ln \tilde{i}$ & $\theta-\infty \vec{m} \stackrel{\infty}{-}$ & \#or & $\stackrel{\infty}{N}-n \stackrel{n}{-} \underset{0}{\alpha}$ & 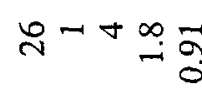 \\
\hline 8 & 의요 & 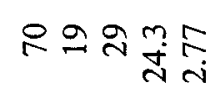 & $\stackrel{\infty}{=} \stackrel{\infty}{\sim} \underset{n}{n} \stackrel{0}{n}$ & শ্ণ & $\stackrel{\sim}{2} \because \underset{\dot{n}}{\sim}$ \\
\hline 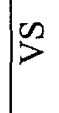 & 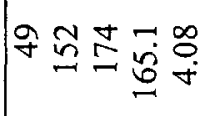 & ชู음ำ & 늄ㅇㅇㅛ & 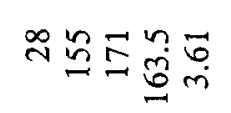 & 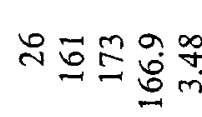 \\
\hline 2 & $\ddot{n}=\bar{\sim} \stackrel{9}{9}$ & 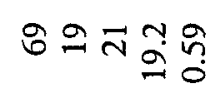 & $\cong \approx \bar{N} \stackrel{m}{2}$ & 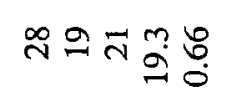 & 뉴류 $\underset{\infty}{\infty} \overline{0}$ \\
\hline$\sum_{i}^{2}$ & 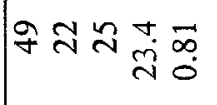 & กํำ & 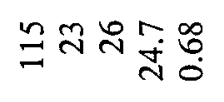 & 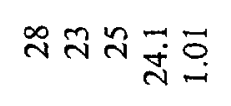 & 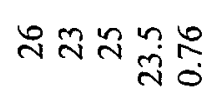 \\
\hline 告 & F & 的忩西 & g̊ㅁㄲㅁㅇㅝ & $\stackrel{\sim}{\sim} \bar{m} \underset{\sim}{\stackrel{n}{n}}$ & 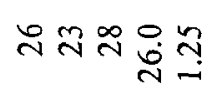 \\
\hline 惫 & 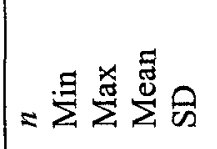 & 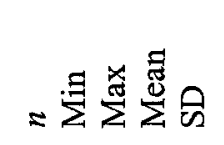 & 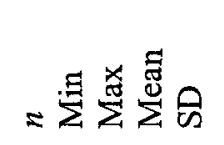 & 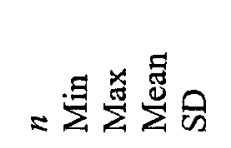 & 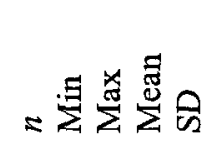 \\
\hline .0. & $\sum_{U}^{\leftrightarrows}$ & $\vec{I}$ & 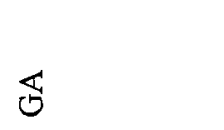 & $\stackrel{5}{4}$ & $\underset{G}{\leftrightarrows}$ \\
\hline
\end{tabular}




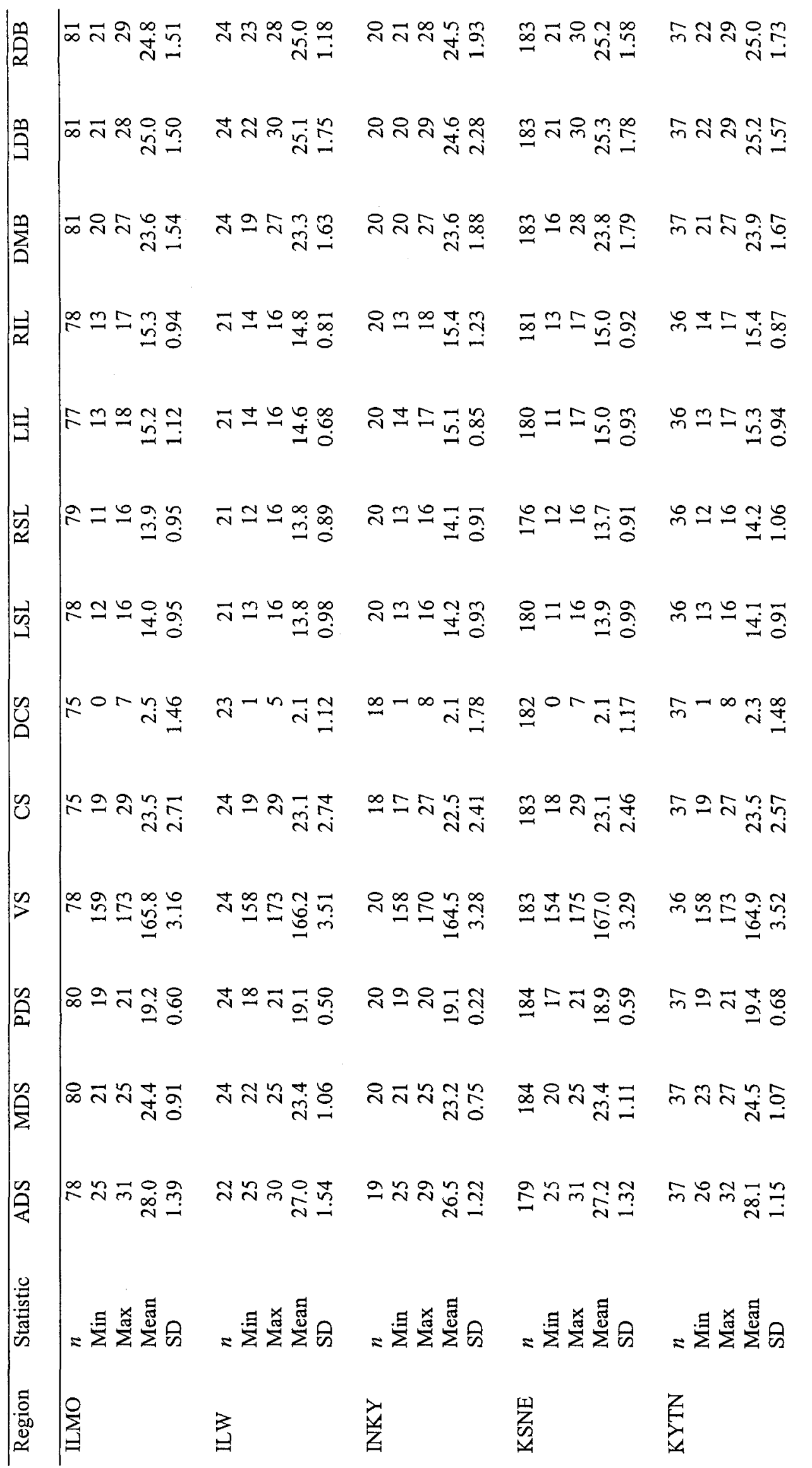




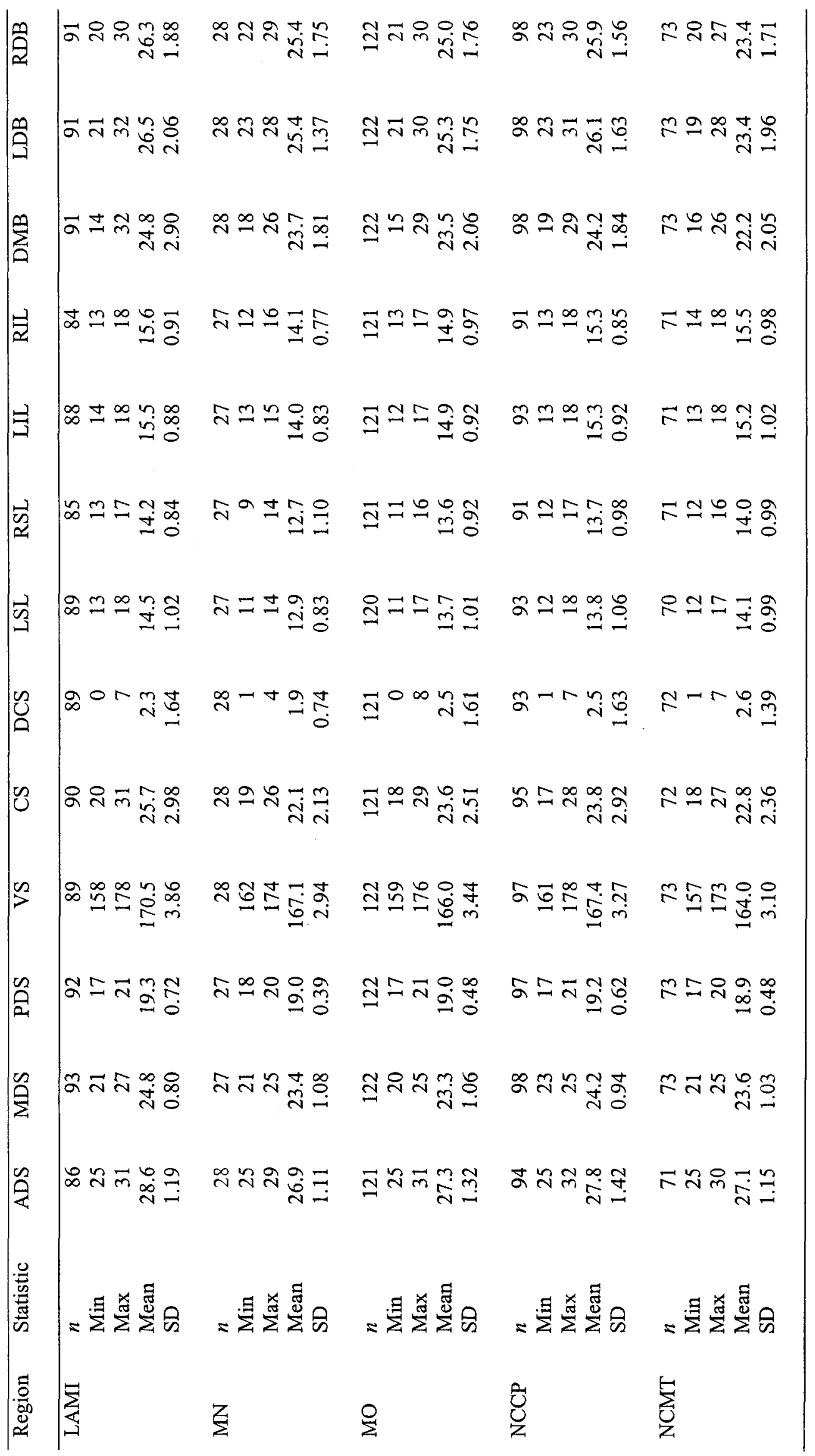




\begin{tabular}{|c|c|c|c|c|c|}
\hline 舀 & 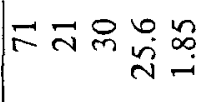 & 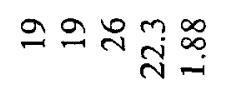 & 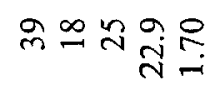 & 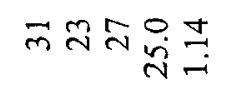 & 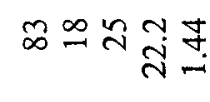 \\
\hline 䱎 & 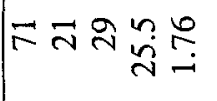 & 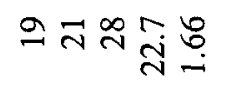 & & m & 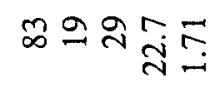 \\
\hline$\sum_{0}^{\infty}$ & 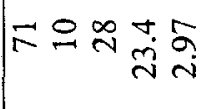 & এニさ욤 & 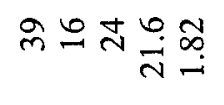 & 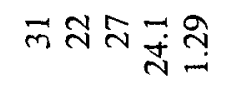 & ஸ્ડ \\
\hline 童 & $\ddot{b}=\infty \frac{m}{n} \underset{0}{2}$ & 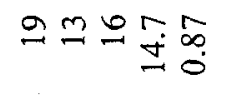 & 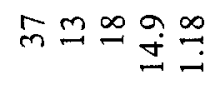 & 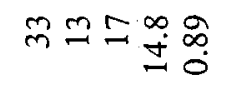 & 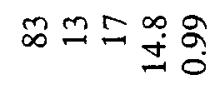 \\
\hline 寻 & 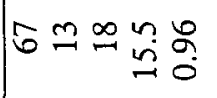 & 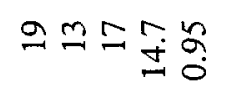 & 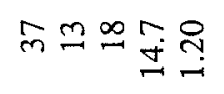 & 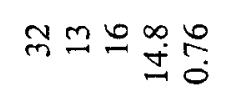 & 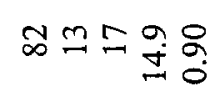 \\
\hline 党 & 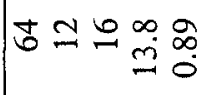 & 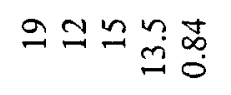 & 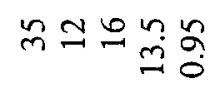 & 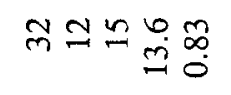 & 표유 \\
\hline 氠 & 62000 & 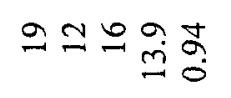 & & 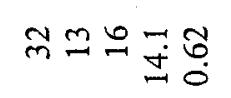 & 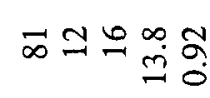 \\
\hline $\begin{array}{l}n \\
0 \\
0\end{array}$ & 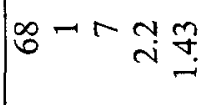 & 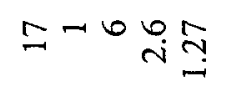 & $q-0 \underset{i}{0} \stackrel{n}{n}$ & $m-\infty \stackrel{\infty}{m} \stackrel{\infty}{\stackrel{\infty}{\Omega}}$ & 임 \\
\hline 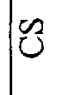 & 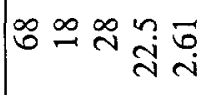 & ニニヘ & 우소슈요 & $m \vec{m} \stackrel{\infty}{\infty} \underset{\sim}{\sim} \stackrel{\infty}{\sim}$ & 초유요 \\
\hline$i s$ & 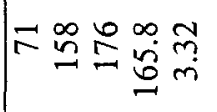 & 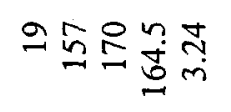 & +oom & mढ゙ & 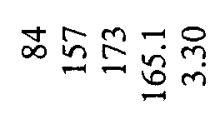 \\
\hline 告 & $\vec{\nabla} \vec{\sim} \vec{g}$ & এニ & 으유용 & $m=\stackrel{m}{m} \underset{\infty}{\infty} \stackrel{\infty}{\stackrel{\infty}{0}}$ & I \\
\hline 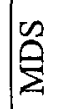 & 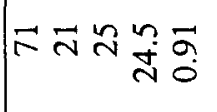 & 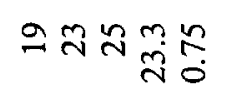 & 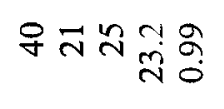 & 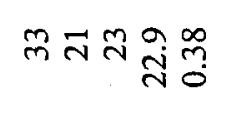 & म \\
\hline$\frac{2}{2}$ & 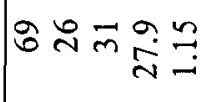 & 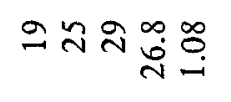 & 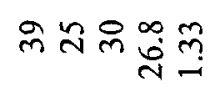 & 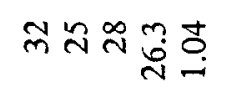 & 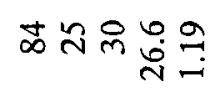 \\
\hline : & 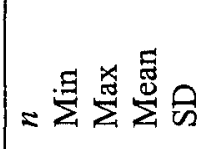 & 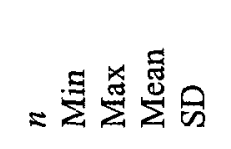 & 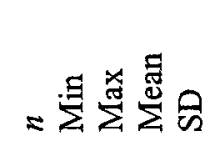 & 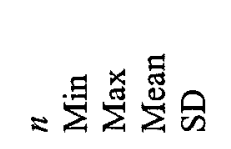 & 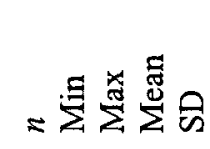 \\
\hline : & 离 & 它 & 之 & $z$ & 岂 \\
\hline
\end{tabular}




\begin{tabular}{|c|c|c|c|c|c|}
\hline$\stackrel{m}{\beta}$ & 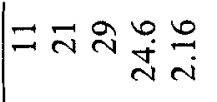 & 舟綮志 & 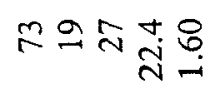 & 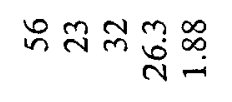 & 읓ำ \\
\hline$\stackrel{m}{\beta}$ & 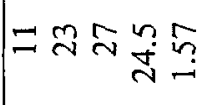 & 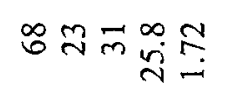 & 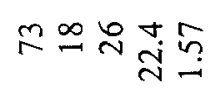 & ウ̊ & 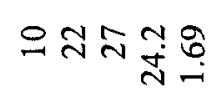 \\
\hline$\sum_{i}^{n}$ & $=9$ 월 & 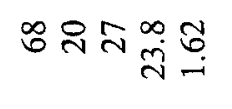 & m in 이용 & 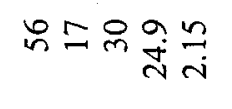 & 음뮤 \\
\hline 国 & $= \pm 0=\frac{R}{2}$ & 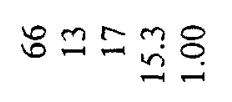 & 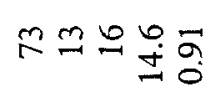 & 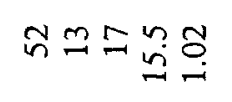 & $a \cong=\stackrel{0}{0}$ \\
\hline 是 & $=20 \stackrel{9}{ \pm} 8$ & 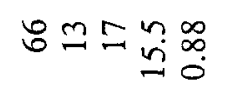 & ホニニ过㐫 & 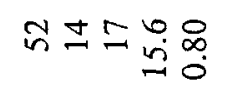 & 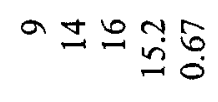 \\
\hline $\overrightarrow{\mathscr{2}}$ & $=m \approx \frac{\infty}{m} \stackrel{n}{0}$ & $\hat{\sigma}=0 \sigma \hat{\sigma}$ & 푹유 & 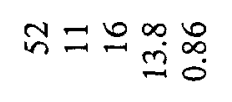 & $a \cong \pm \underset{j}{2}$ \\
\hline $\overrightarrow{0}$ & 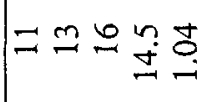 & 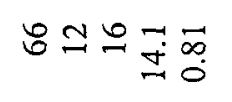 & $\Re \simeq \infty \underset{\sim}{\sim}$ & $\vec{n} \simeq 0 \stackrel{a}{m}$ & 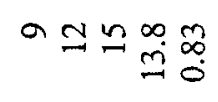 \\
\hline $\mathscr{U}_{0}$ & $=-\nabla \stackrel{n}{=}$ & 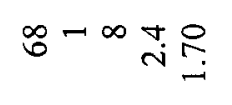 & ホーN管 & 芯品莳 & $a-m \stackrel{m}{-}$ \\
\hline$\tilde{z}$ & 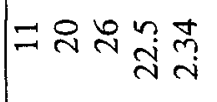 & 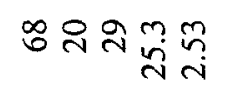 & 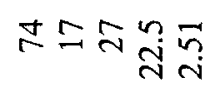 & 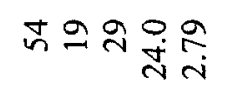 & 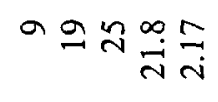 \\
\hline$p$ & 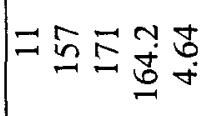 & 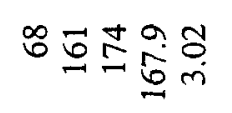 & 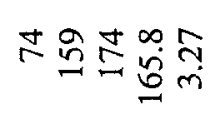 & 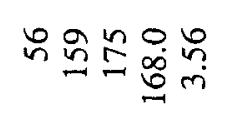 & 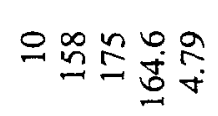 \\
\hline 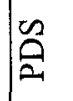 & $=9908$ & 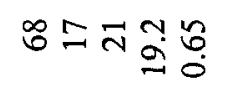 & ホニ & inএ & 으으응ㅇㅇㅇ \\
\hline$\stackrel{2}{\Sigma}$ & 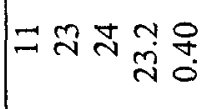 & 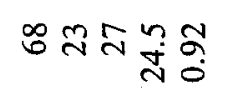 & 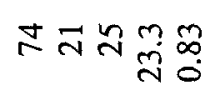 & 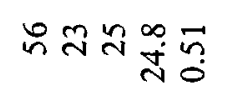 & 음 \\
\hline 丝 & $=\approx$ 웡ㅁㅇ & 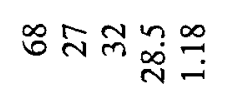 & 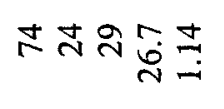 & ెㅠ 유 N $\underset{\infty}{m}$ & 으뉴유: \\
\hline $\mid \frac{0}{0}$ & 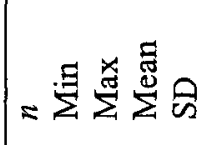 & 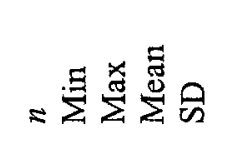 & 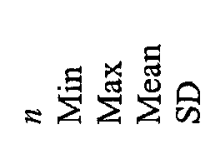 & 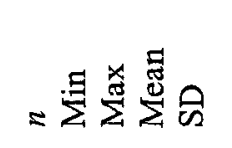 & 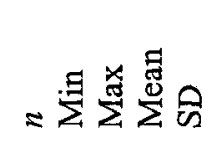 \\
\hline . & 宊 & 峁 & 䆓 & $\underset{\infty}{U}$ & 乬 \\
\hline
\end{tabular}




\begin{tabular}{|c|c|c|c|c|}
\hline$\stackrel{\rho}{\nexists}$ & 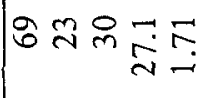 & 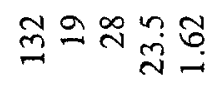 & లี సิ & 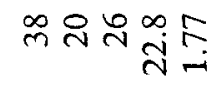 \\
\hline 絪 & 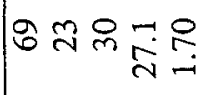 & 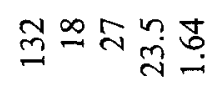 & 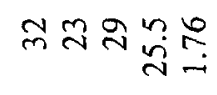 & mo 유 \\
\hline$\sum_{A}^{n}$ & 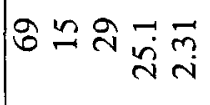 & 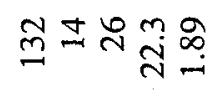 & 교요 & 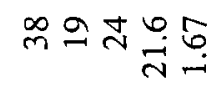 \\
\hline 골 & m士ニ芯志 & 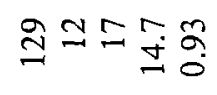 & $m \simeq \because \underset{ \pm}{\dot{m}}$ & m \\
\hline 寻 & 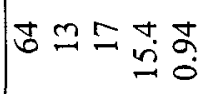 & 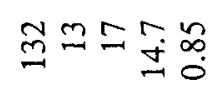 & 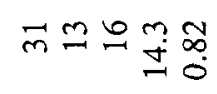 & 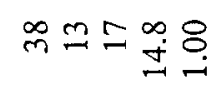 \\
\hline 焉 & $\ddot{b}=0 \stackrel{9}{ \pm}$ & 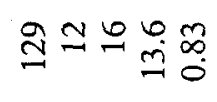 & 크청 & 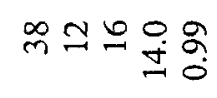 \\
\hline 勻 & 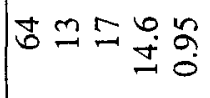 & 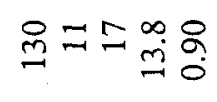 & 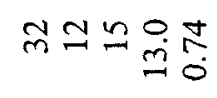 & 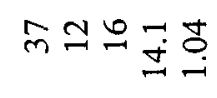 \\
\hline $\begin{array}{l}\mathscr{L} \\
0 \\
0\end{array}$ & $s^{-\infty} \tilde{i} \stackrel{\infty}{?}$ & 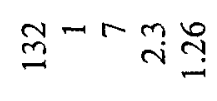 & $m-n g .9$ & $\stackrel{\infty}{m}-n \vec{i}=$ \\
\hline 8 & চি유 $\vec{\sim}$ & 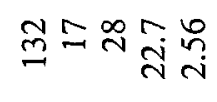 & 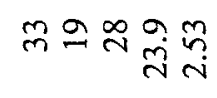 & mి \\
\hline$\infty$ & 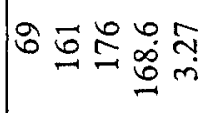 & 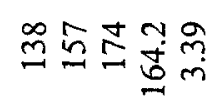 & 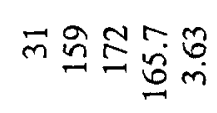 & 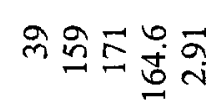 \\
\hline $\mathscr{2}_{2}$ & $\theta \stackrel{2}{0} \stackrel{n}{a}$ & $\stackrel{\rho}{=}=\vec{\sim} \underset{0}{\infty} \overrightarrow{0}_{0}^{\infty}$ & 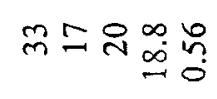 & 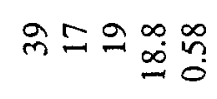 \\
\hline$\hat{\Sigma}$ & Sন & 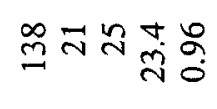 & 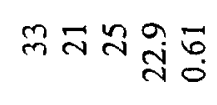 & 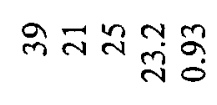 \\
\hline$\frac{n}{a}$ & 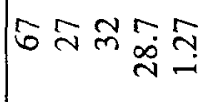 & 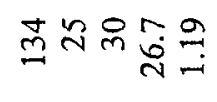 & " & 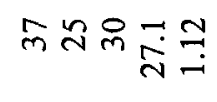 \\
\hline 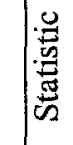 & 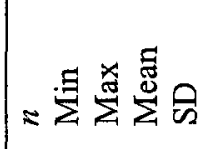 & 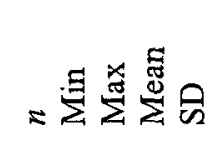 & 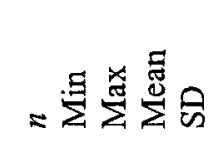 & 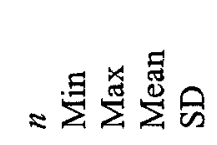 \\
\hline 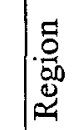 & $x$ & $\sum_{S}^{S}$ & 5 & $\sum_{3}^{2}$ \\
\hline
\end{tabular}




\begin{tabular}{|c|c|c|c|c|c|}
\hline $\mid \begin{array}{l}3 \\
\frac{5}{2}\end{array}$ & $\mid \hat{\infty}_{0} 0 \forall \underset{m}{\vec{m}} \underset{0}{\infty}$ & mం & mm॰ & gon & $\sin \underset{m}{m} \stackrel{0}{0}$ \\
\hline ص్m: & 궇 & 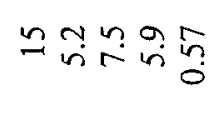 & 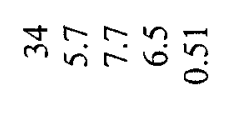 & 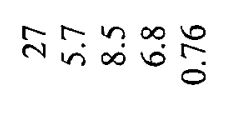 & 꿍 \\
\hline 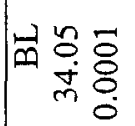 & $\alpha \sim \forall \stackrel{\sim}{\sim} \underset{0}{0}$ & 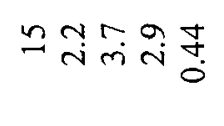 & $\vec{m} \sim \vec{r} \tilde{i} \overrightarrow{0}$ & ơ & NN 年 \\
\hline$\underbrace{\infty}_{i} \leq$ & NOOl & non & mond & fon 목 & तonm \\
\hline 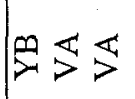 & Noming & no- & 苾o-m 管 & 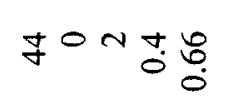 & הం- \\
\hline$\rho \$$ & No $0-\overrightarrow{0} \underset{0}{\tilde{0}}$ & no- & ț00 : $:$ & no- - & ה̃-0 융 \\
\hline 色せ & NoaI욤 & nం & प్mంn & 䢴 & त् \\
\hline Mr & $\alpha$ 거 & $\because$ ఇ & 芦光品芯导 & ที่ & 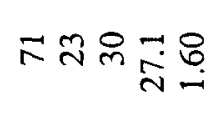 \\
\hline 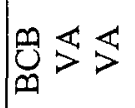 & 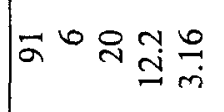 & $\because \infty=\stackrel{\text { తa }}{ }$ & 出 & 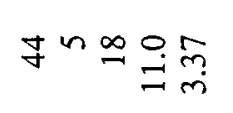 & $\approx+g \underset{j}{0} \underset{m}{m}$ \\
\hline 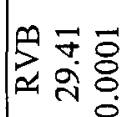 & 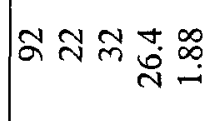 & 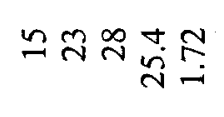 & "ాసন స్తి & $\forall \bar{\sim} \underset{\sim}{\infty} \stackrel{\infty}{\sim} \stackrel{\infty}{=}$ & 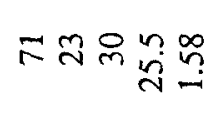 \\
\hline 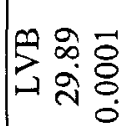 & 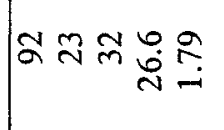 & 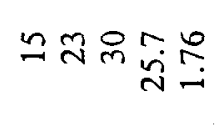 & 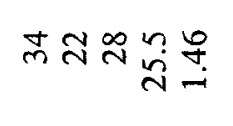 & 㘶 & 주 \\
\hline 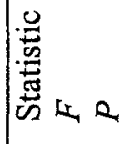 & 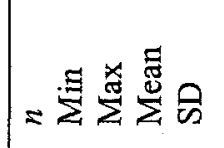 & 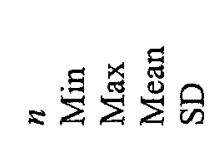 & 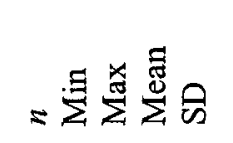 & 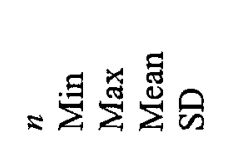 & 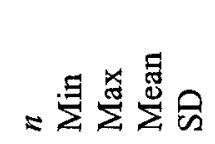 \\
\hline 蒿 & $\sum_{4}$ & 岕 & 希 & $\sum_{i=}^{\mathbb{U}}$ & 㱏 \\
\hline
\end{tabular}




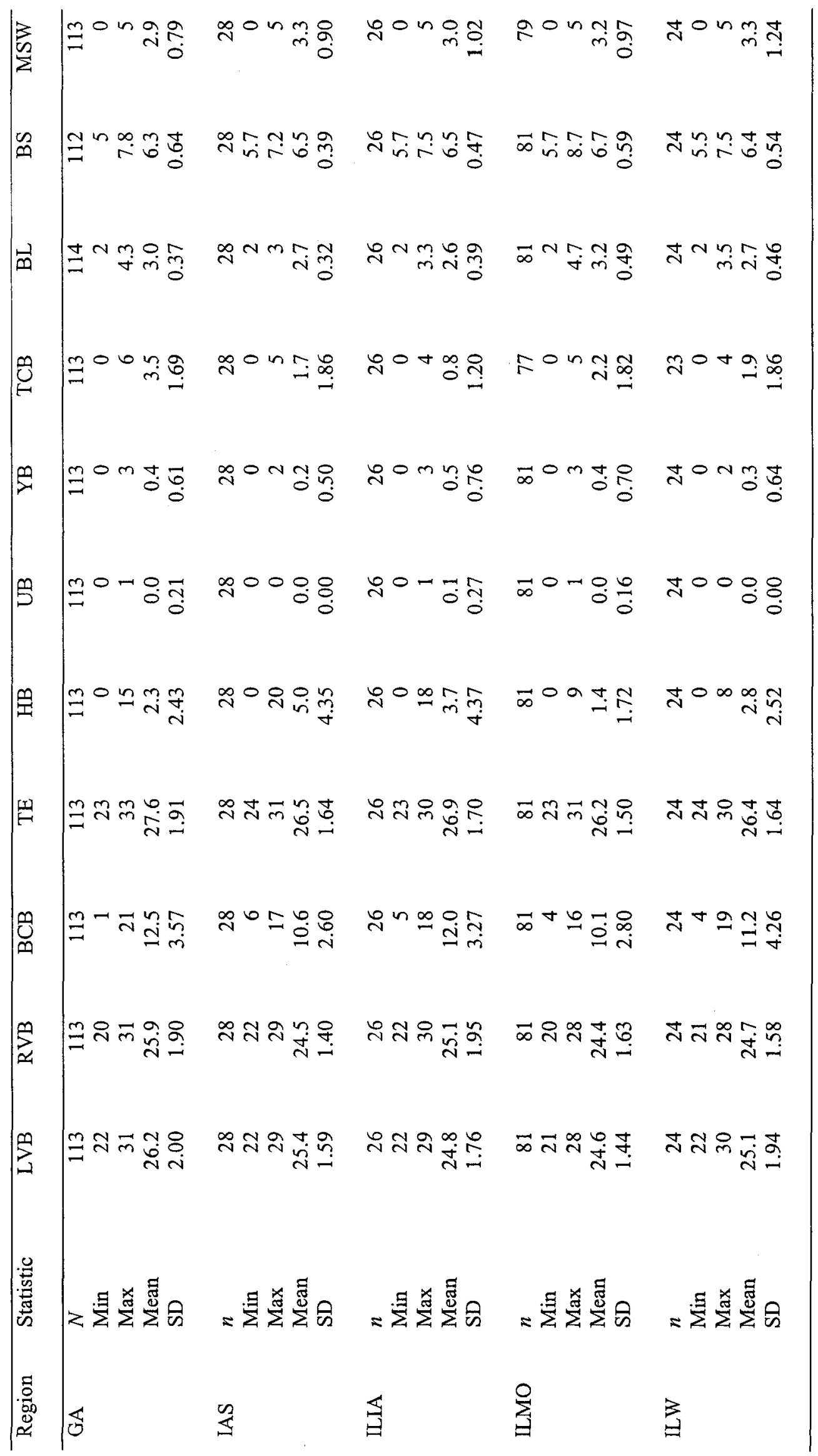




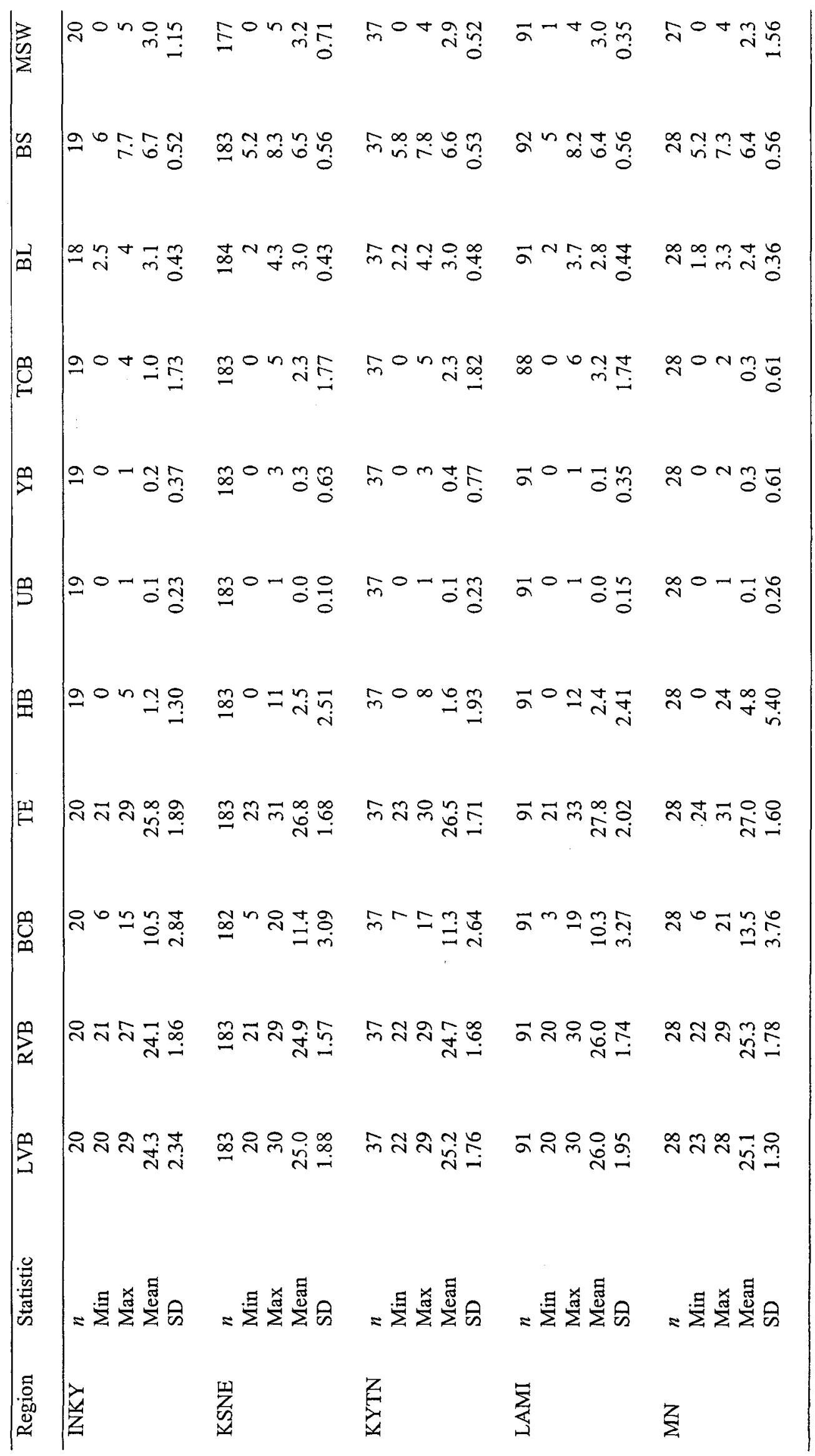




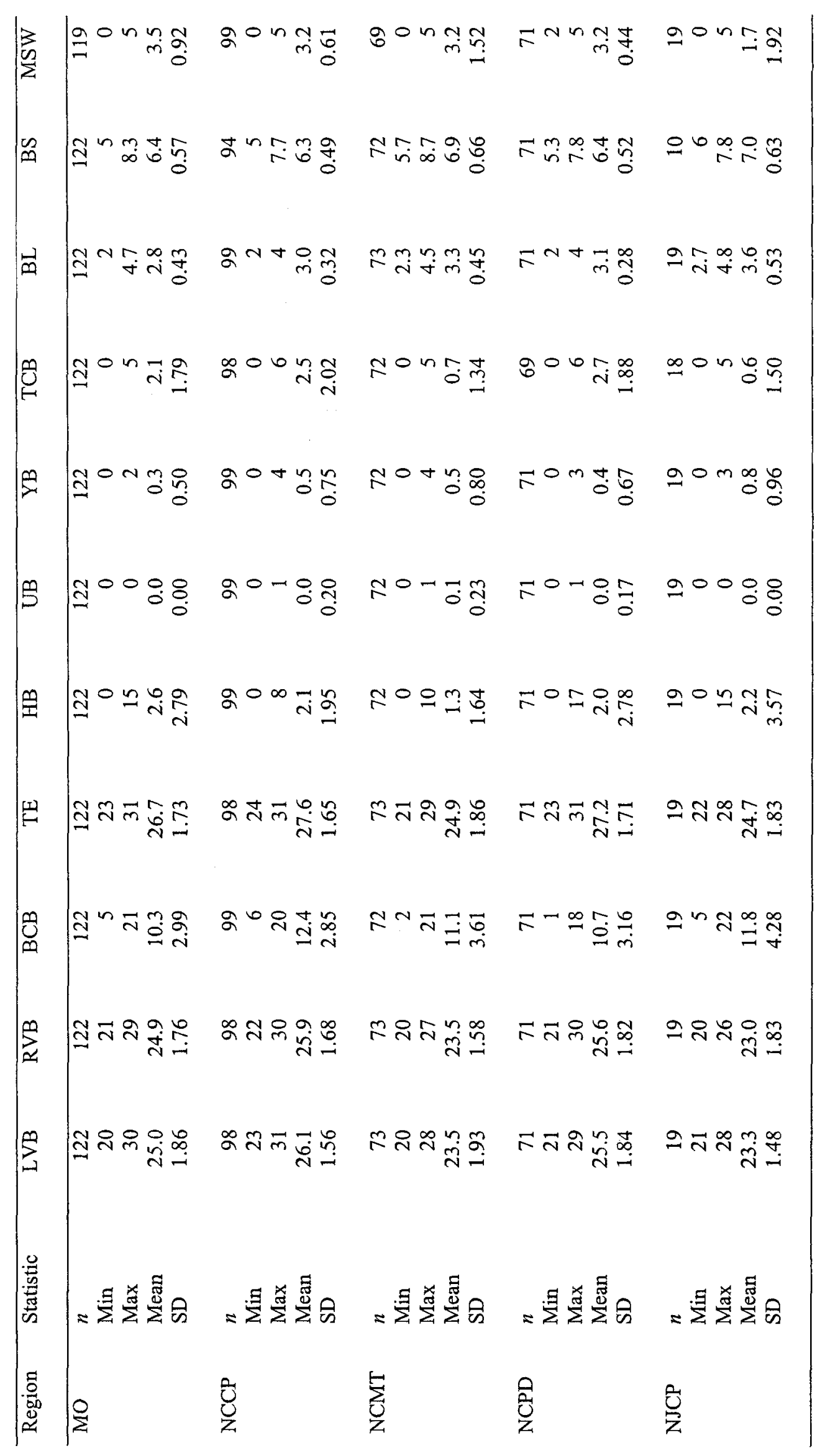




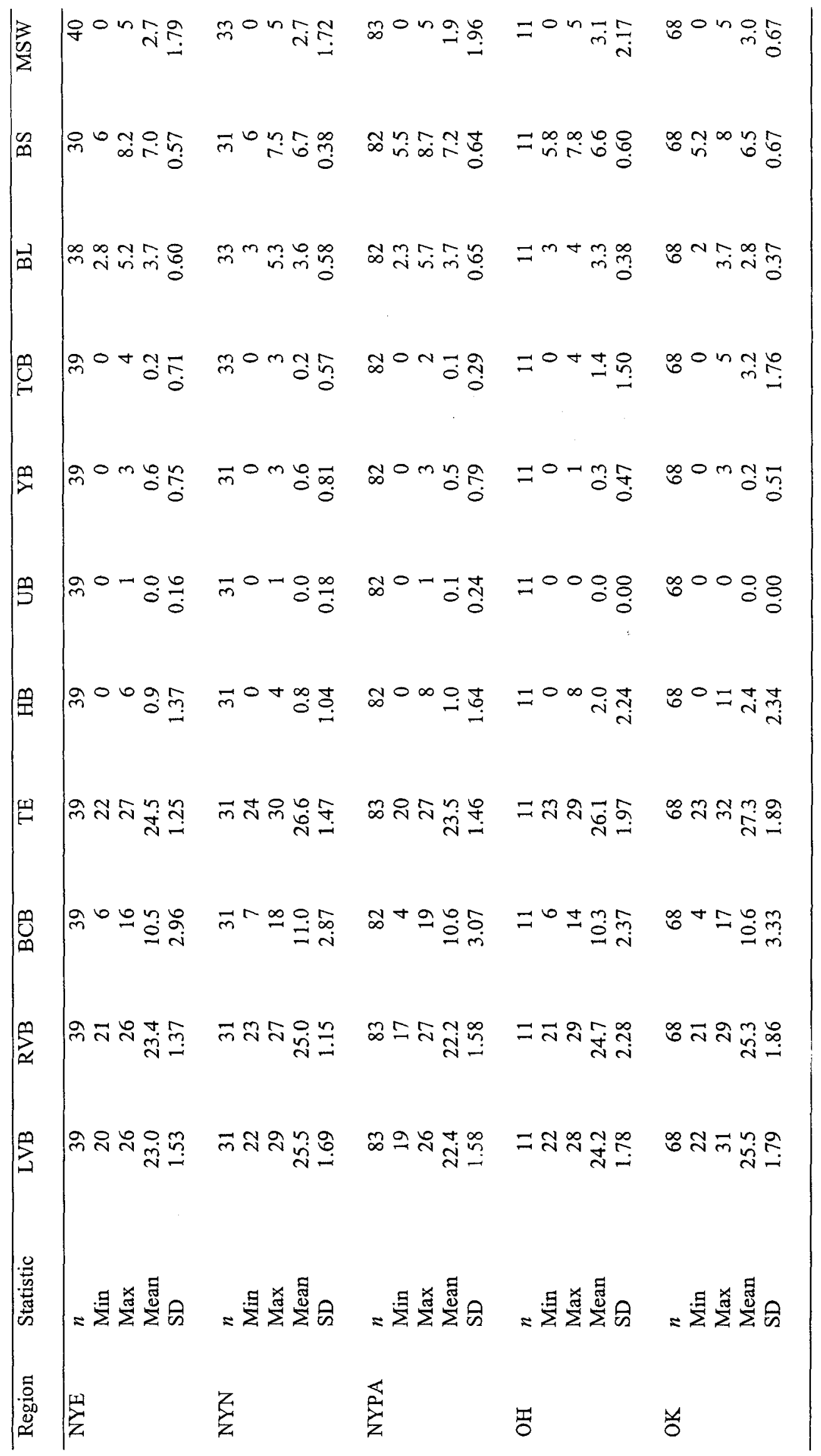




\begin{tabular}{|c|c|c|c|c|c|}
\hline$\sum_{i}^{\infty}$ & ㄱon & non & 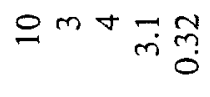 & $\ddot{b} \sim \backsim \stackrel{0}{m}$ & $\Omega_{n}^{\circ}$ \\
\hline$\stackrel{\sim}{\infty}$ & 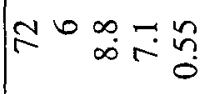 & 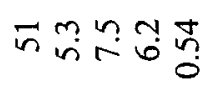 & 으 ํㅜㅇ & 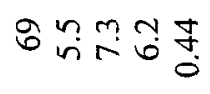 & 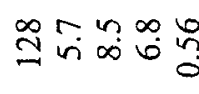 \\
\hline$\vec{\oplus}$ & I & ㅁํ & 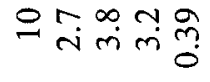 & gेल & $m^{\infty} \tilde{n}^{\circ} m b$ \\
\hline 0 & (ton m & 共ol & 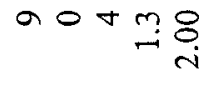 & bovr & 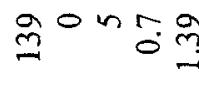 \\
\hline$\sum_{\gamma}^{\infty}$ & mom & 吕 & 으융 & gे० $\overrightarrow{0}$ ? & $\underset{m}{m} \circ \stackrel{0}{0} \stackrel{0}{0}$ \\
\hline$\frac{9}{\rho}$ & mon $\overrightarrow{0} \overrightarrow{0}$ & 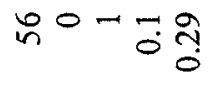 & 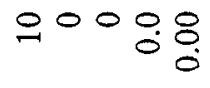 & g⿵人一 & $m^{m} \circ-\vec{n}$ \\
\hline 国 & mog & non & 엉요 & 응요 & $m_{-1} 0 \vec{v}$ \\
\hline$\stackrel{\text { 니 }}{\oplus}$ & & 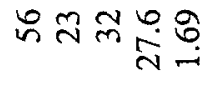 & 어요 & $g \dddot{b} \underset{\sim}{\Delta}$ & 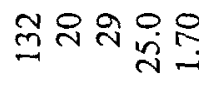 \\
\hline$\bigcup_{\infty}^{\infty}$ & №g $\frac{m}{=}$ & 品学 & 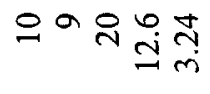 & 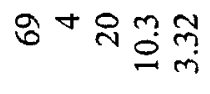 & 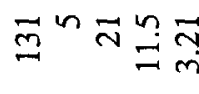 \\
\hline$\sum_{\infty}^{\infty}$ & 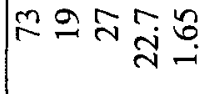 & ウ & 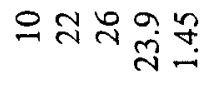 & 合 & $\stackrel{\overbrace{}}{=} \stackrel{\sim}{\sim} \underset{\sim}{\sim}$ \\
\hline$\sum_{1}^{\infty}$ & $m \stackrel{\sim}{\sim} \stackrel{\infty}{\sim} \stackrel{\infty}{\sim}$ & ํㅠㄹ & 으욧ㄷㅇ용 & 그 & $\underset{\sim}{\sim} \stackrel{\infty}{\sim} \underset{\sim}{\sim}$ \\
\hline 总 & 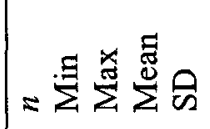 & 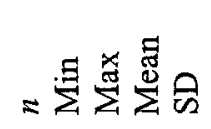 & 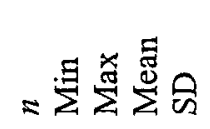 & 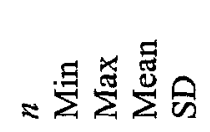 & 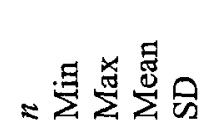 \\
\hline 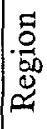 & 㱏 & U & 导 & 虔 & $\sum_{>}^{-5}$ \\
\hline
\end{tabular}




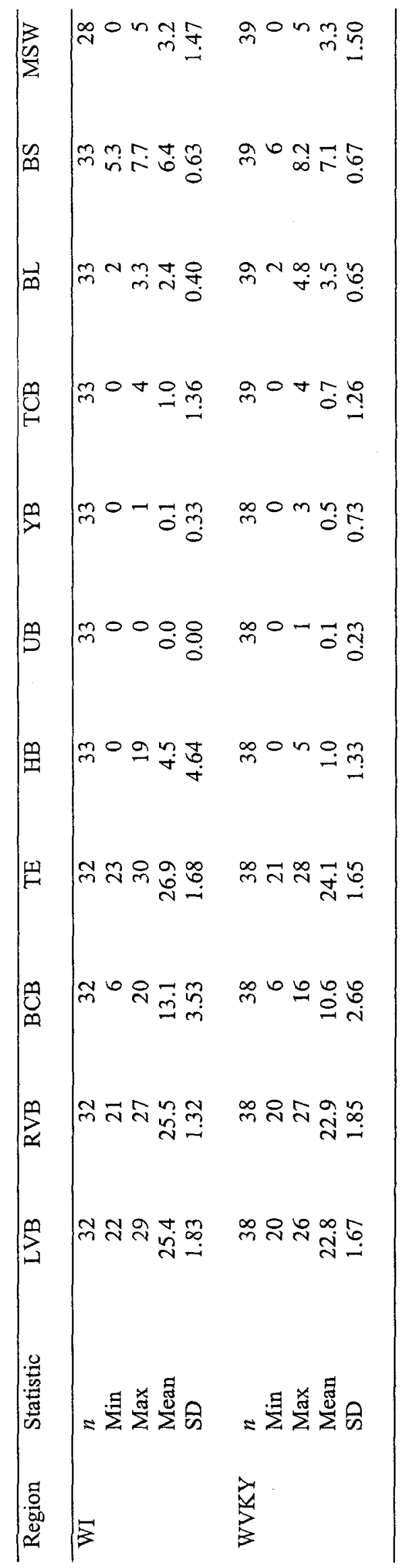




\section{APPENDIX VII}

Table 7.1.-Principal component analysis of 16 meristic variables of males $(n=874)$. Eigenvalues of the covariance matrix and eigenvectors for each principal component are listed. The total variance is 0.10855 .

\begin{tabular}{|c|c|c|c|c|c|c|}
\hline & $P C$ & Eigenvalue & Difference & \multicolumn{2}{|c|}{ Proportion } & ulative \\
\hline & 1 & 0.05249525 & 0.03268153 & \multicolumn{2}{|c|}{0.4836} & 0.4836 \\
\hline & 2 & 0.01981372 & 0.01012679 & \multicolumn{2}{|c|}{0.1825} & 0.6661 \\
\hline & 3 & 0.00968692 & 0.00505428 & \multicolumn{2}{|c|}{0.0892} & 0.7554 \\
\hline & 4 & 0.00463264 & 0.00036031 & \multicolumn{2}{|c|}{0.0427} & 0.7981 \\
\hline & 5 & 0.00427234 & 0.00098836 & \multicolumn{2}{|c|}{0.0394} & 0.8374 \\
\hline & 6 & 0.00328397 & 0.00031982 & \multicolumn{2}{|c|}{0.0303} & 0.8677 \\
\hline & 7 & 0.00296416 & 0.00043985 & \multicolumn{2}{|c|}{0.0273} & 0.8950 \\
\hline & 8 & 0.00252431 & 0.00005384 & \multicolumn{2}{|c|}{0.0233} & 0.9182 \\
\hline & 9 & 0.00247047 & 0.00042666 & \multicolumn{2}{|c|}{0.0228} & 0.9410 \\
\hline & 10 & 0.00204381 & 0.00086049 & \multicolumn{2}{|c|}{0.0188} & 0.9598 \\
\hline & 11 & 0.00118332 & 0.00029587 & \multicolumn{2}{|c|}{0.0109} & 0.9707 \\
\hline & 12 & 0.00088745 & 0.00008107 & \multicolumn{2}{|c|}{0.0082} & 0.9789 \\
\hline & 13 & 0.00080638 & 0.00017112 & \multicolumn{2}{|c|}{0.0074} & 0.9863 \\
\hline & 14 & 0.00063527 & 0.00009846 & & 059 & 0.9922 \\
\hline & 15 & 0.00053681 & 0.00022402 & & 049 & 0.9971 \\
\hline & 16 & 0.00031279 & & & 029 & 1.0000 \\
\hline Char & cter & Prin1 & Prin2 & Prin3 & Prin4 & Prin5 \\
\hline$x 1$ & ADS & 0.060971 & 0.072011 & 0.207954 & 0.145420 & 0.183847 \\
\hline$\times 2$ & MDS & 0.054664 & 0.090587 & 0.144471 & 0.114281 & 0.130571 \\
\hline$x 3$ & PDS & 0.028283 & 0.041317 & 0.068830 & 0.042543 & 0.078857 \\
\hline$x 4$ & vs & 0.025635 & 0.032528 & 0.024438 & 0.095797 & -013779 \\
\hline$x 5$ & $\operatorname{cs}$ & 0.101133 & 0.077863 & 0.143819 & 0.575250 & 0.100115 \\
\hline$x 7$ & LSL & 0.029748 & 0.093051 & 0.512083 & -.063105 & -.456803 \\
\hline$\times 8$ & RSL & 0.020201 & 0.092256 & 0.508085 & -.108488 & -.473915 \\
\hline$\times 9$ & LIL & 0.052067 & 0.140010 & 0.370479 & -.147966 & 0.514307 \\
\hline$x 10$ & RIL & 0.053238 & 0.095566 & 0.421852 & -.122266 & 0.445934 \\
\hline$x 12$ & LDB & 0.309400 & 0.268664 & - . 097997 & 0.095756 & -107879 \\
\hline$\times 13$ & RDB & 0.304322 & 0.258549 & -.067359 & 0.145344 & -.001283 \\
\hline$\times 14$ & LVB & 0.295328 & 0.289543 & -106991 & -.003122 & -.133055 \\
\hline$\times 15$ & RVB & 0.283168 & 0.256513 & -.088133 & 0.107716 & 0.037981 \\
\hline$\times 17$ & $\mathrm{TE}$ & 0.288259 & 0.266433 & -.099663 & 0.136060 & -.049124 \\
\hline$x 22$ & BL & -.662261 & 0.736038 & -.111298 & -.032392 & -.003056 \\
\hline$x 23$ & BS & -.312003 & -.174570 & 0.136366 & 0.714024 & -.053648 \\
\hline
\end{tabular}




\begin{tabular}{|c|c|c|c|c|c|c|c|}
\hline & Prin6 & Prin7 & \multicolumn{2}{|c|}{ Prin8 } & Pring & Prin10 & Prin11 \\
\hline$x 1$ & -.189168 & 0.081486 & \multicolumn{2}{|c|}{0.254090} & 0.550878 & 0.265243 & -.612655 \\
\hline$x 2$ & -.088707 & 0.050801 & \multicolumn{2}{|c|}{0.139559} & 0.415638 & 0.265097 & 0.768745 \\
\hline$x 3$ & -.075456 & -.008425 & \multicolumn{2}{|c|}{0.083410} & 0.166017 & 0.172171 & 0.090759 \\
\hline$x 4$ & -.009477 & 0.019452 & \multicolumn{2}{|c|}{0.025897} & -.017495 & -.025913 & 0.019364 \\
\hline$x 5$ & -.671504 & -.131523 & \multicolumn{2}{|c|}{-119222} & -.277300 & -.227776 & 0.038031 \\
\hline$x 7$ & 0.028662 & -193353 & \multicolumn{2}{|c|}{-.645538} & 0.223192 & 0.076193 & -.041458 \\
\hline$x 8$ & -.016457 & 0.072883 & \multicolumn{2}{|c|}{0.642582} & -.202850 & -.170768 & 0.043597 \\
\hline$\times 9$ & 0.192482 & 0.193737 & \multicolumn{2}{|c|}{-.106315} & 0.161556 & -.660291 & 0.030600 \\
\hline$\times 10$ & 0.124773 & -.034299 & \multicolumn{2}{|c|}{-.056695} & -.534610 & 0.534926 & -.036870 \\
\hline$x 12$ & 0.009476 & 0.447377 & \multicolumn{2}{|c|}{-.050650} & -097771 & 0.028080 & -.109829 \\
\hline$\times 13$ & 0.245002 & -.398731 & \multicolumn{2}{|c|}{0.120300} & -.003857 & -.056676 & -.031703 \\
\hline$\times 14$ & 0.024744 & 0.517764 & \multicolumn{2}{|c|}{-.157560} & -.062275 & 0.067716 & 0.063878 \\
\hline$\times 15$ & 0.265615 & -.472816 & \multicolumn{2}{|c|}{0.089493} & 0.027648 & -.075085 & -.006273 \\
\hline$x 17$ & 0.132765 & 0.010247 & & & 0.029871 & 0.072026 & -.002912 \\
\hline$x 22$ & -.060684 & -.036547 & & 305 & -.014130 & 0.013277 & -.019373 \\
\hline$\times 23$ & 0.541615 & 0.198535 & & 509 & -.028895 & 0.022102 & -.011837 \\
\hline & Prin12 & Pri & & & in14 & Prin15 & Prin16 \\
\hline$x 1$ & 0.021690 & -.174 & & 0.0 & 0944 & 0.022847 & -.019660 \\
\hline$\times 2$ & -.120014 & -.214 & & 0.0 & 4122 & -.106778 & -.038932 \\
\hline$\times 3$ & 0.121521 & 0.929 & & 0.0 & 9408 & 0.158774 & 0.053858 \\
\hline$x 4$ & -.019578 & -.064 & & 0.0 & 4416 & 0.028155 & 0.986599 \\
\hline$\times 5$ & 0.048950 & -.015 & & 0.0 & 1989 & 0.025849 & -.077391 \\
\hline$x 7$ & -.042422 & 0.017 & & -.0 & 3322 & -.032155 & 0.012536 \\
\hline$x 8$ & 0.064962 & 0.001 & & -.0 & 6291 & 0.002835 & -.036941 \\
\hline$\times 9$ & 0.007740 & 0.042 & 48 & -.0 & 5274 & 0.014200 & -.000605 \\
\hline$\times 10$ & -.003071 & -.052 & 77 & 0.0 & 2518 & -.012779 & 0.008441 \\
\hline$x 12$ & -.481736 & 0.182 & 80 & 0.0 & 0736 & -.557735 & -.013331 \\
\hline$\times 13$ & -.570850 & -.010 & & 0.0 & 2359 & 0.498757 & -.049206 \\
\hline$x 14$ & 0.323287 & -.111 & & 0.3 & 3642 & 0.465798 & -.070350 \\
\hline$\times 15$ & 0.406034 & 0.014 & 48 & 0.4 & 9275 & -.427639 & -.030051 \\
\hline$\times 17$ & 0.364642 & -.066 & & -.8 & 7148 & 0.009740 & 0.047678 \\
\hline$x 22$ & -019885 & -.002 & & -.0 & 3697 & -.005236 & -.000674 \\
\hline$x 23$ & 0.028519 & 0.024 & 40 & 0.0 & 7883 & 0.014445 & -.059262 \\
\hline
\end{tabular}


Table 7.2.-Principal component analysis of 16 meristic variables of females $(n=751)$. Eigenvalues of the covariance matrix and eigenvectors for each principal component are listed. The total variance is 0.10420 .

\begin{tabular}{|c|c|c|c|c|c|c|}
\hline & $P C$ & Eigenvalue & Difference & \multicolumn{2}{|c|}{ Proportion } & ulative \\
\hline & 1 & 0.04847775 & 0.03064791 & \multicolumn{2}{|c|}{0.4653} & 0.4653 \\
\hline & 2 & 0.01782984 & 0.00835933 & \multicolumn{2}{|c|}{0.1711} & 0.6364 \\
\hline & 3 & 0.00947051 & 0.00453300 & \multicolumn{2}{|c|}{0.0909} & 0.7273 \\
\hline & 4 & 0.00493751 & 0.00084396 & \multicolumn{2}{|c|}{0.0474} & 0.7747 \\
\hline & 5 & 0.00409355 & 0.00024584 & \multicolumn{2}{|c|}{0.0393} & 0.8139 \\
\hline & 6 & 0.00384771 & 0.00059848 & \multicolumn{2}{|c|}{0.0369} & 0.8509 \\
\hline & 7 & 0.00324923 & 0.00032036 & \multicolumn{2}{|c|}{0.0312} & 0.8821 \\
\hline & 8 & 0.00292887 & 0.00022809 & \multicolumn{2}{|c|}{0.0281} & 0.9102 \\
\hline & 9 & 0.00270078 & 0.00058205 & \multicolumn{2}{|c|}{0.0259} & 0.9361 \\
\hline & 10 & 0.00211873 & 0.00086745 & \multicolumn{2}{|c|}{0.0203} & 0.9564 \\
\hline & 11 & 0.00125128 & 0.00030547 & \multicolumn{2}{|c|}{0.0120} & 0.9684 \\
\hline & 12 & 0.00094581 & 0.00008237 & \multicolumn{2}{|c|}{0.0091} & 0.9775 \\
\hline & 13 & 0.00086344 & 0.00022491 & \multicolumn{2}{|c|}{0.0083} & 0.9858 \\
\hline & 14 & 0.00063853 & 0.00010717 & & 061 & 0.9919 \\
\hline & 15 & 0.00053136 & 0.00022119 & & 051 & 0.9970 \\
\hline & 16 & 0.00031017 & & & 030 & 1.0000 \\
\hline Chare & cter & Prin1 & Prin2 & Prin3 & Prin4 & Prin5 \\
\hline$x 1$ & ADS & 0.047256 & 0.119538 & 0.160916 & 0.054952 & -.091511 \\
\hline$x 2$ & MDS & 0.059475 & 0.110600 & 0.131291 & 0.004898 & -.106979 \\
\hline$x 3$ & PDS & 0.031236 & 0.040142 & 0.065558 & -.016709 & -.070834 \\
\hline$\times 4$ & VS & 0.023828 & 0.033233 & 0.010222 & 0.065570 & 0.019258 \\
\hline$x 5$ & $\operatorname{cs}$ & 0.114128 & 0.121331 & 0.131828 & 0.598071 & -.593020 \\
\hline$x 7$ & LSL & 0.017644 & 0.219268 & 0.401317 & -.060916 & 0.323435 \\
\hline$x 8$ & RSL & 0.018565 & 0.207446 & 0.438325 & -.174302 & 0.316523 \\
\hline$\times 9$ & LIL & 0.046367 & 0.203994 & 0.408937 & 0.013538 & -199252 \\
\hline$\times 10$ & RIL & 0.052155 & 0.218289 & 0.416697 & -.181240 & -.213215 \\
\hline$x+2$ & LDB & 0.325530 & 0.256670 & -.151561 & 0.027960 & -.021950 \\
\hline$\times 13$ & $\mathrm{RDB}$ & 0.326974 & 0.229860 & -.112214 & 0.145889 & 0.212021 \\
\hline$\times 14$ & LVB & 0.307454 & 0.240346 & -.156561 & 0.000458 & -.022115 \\
\hline$\times 15$ & RVB & 0.296051 & 0.210679 & -.173338 & 0.116406 & 0.257828 \\
\hline$x 17$ & $\mathrm{TE}$ & 0.319909 & 0.229138 & -.157914 & 0.084836 & 0.134699 \\
\hline$x 22$ & $B L$ & -.616733 & 0.707754 & -.318130 & -.074734 & -.083373 \\
\hline$\times 23$ & BS & -.310447 & -.037137 & 0.157398 & 0.720533 & 0.442343 \\
\hline
\end{tabular}




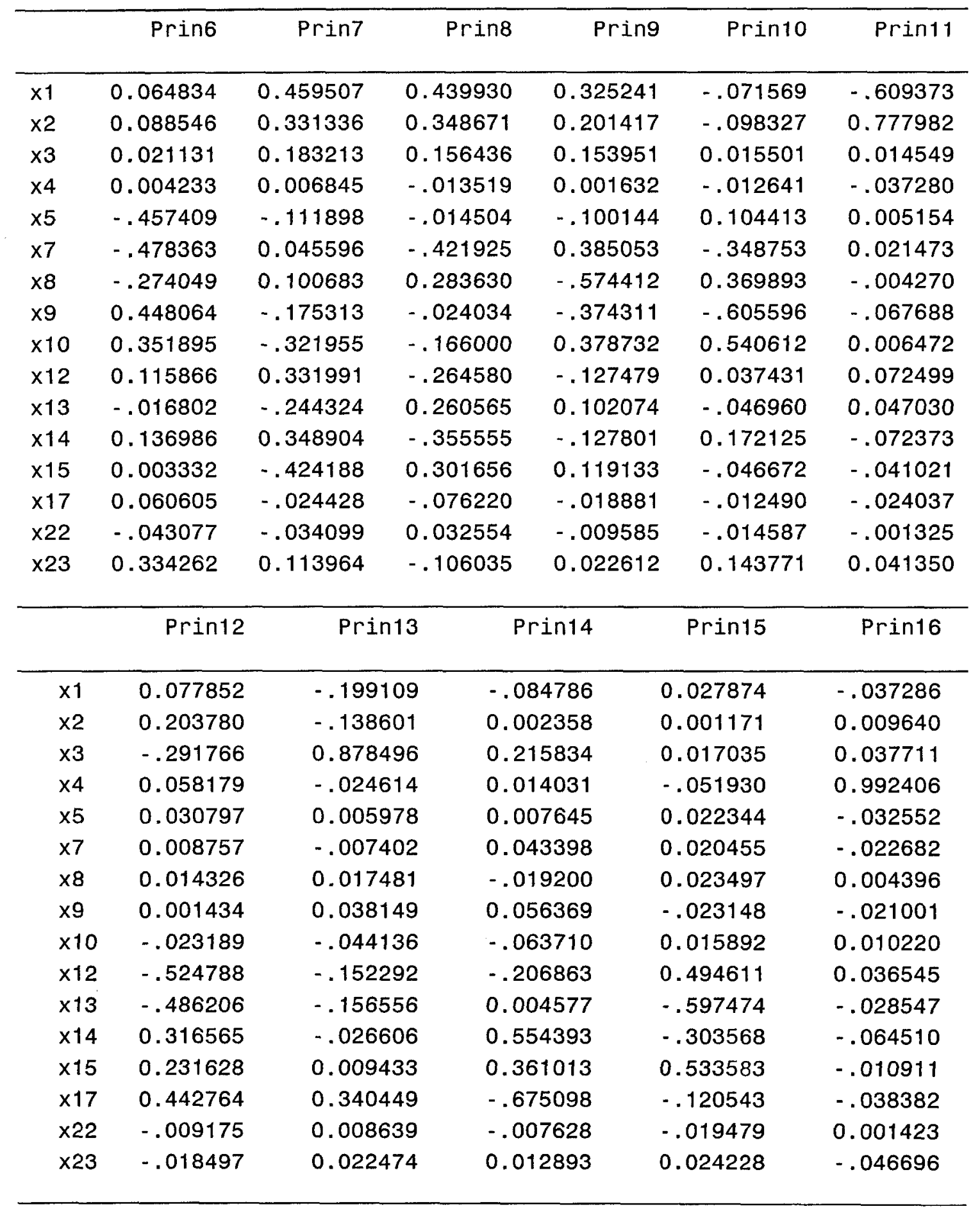


Table 7.3.-Principal component analysis of 16 meristic variables of combined sexes $(n=1626)$. Eigenvalues of the covariance matrix and eigenvectors for each principal component are listed. The total variance is 0.11918 .

\begin{tabular}{|c|c|c|c|c|c|c|}
\hline & $\mathrm{PC}$ & Eigenvalue & Difference & \multicolumn{2}{|c|}{ Proportion } & Cumulative \\
\hline & 1 & 0.05381335 & 0.03439440 & \multicolumn{2}{|c|}{0.4515} & 0.4515 \\
\hline & 2 & 0.01941895 & 0.00667414 & \multicolumn{2}{|c|}{0.1629} & 0.6145 \\
\hline & 3 & 0.01274480 & 0.00311316 & \multicolumn{2}{|c|}{0.1069} & 0.7214 \\
\hline & 4 & 0.00963165 & 0.00520821 & \multicolumn{2}{|c|}{0.0808} & 0.8022 \\
\hline & 5 & 0.00442344 & 0.00043264 & \multicolumn{2}{|c|}{0.0371} & 0.8394 \\
\hline & 6 & 0.00399080 & 0.00090004 & \multicolumn{2}{|c|}{0.0335} & 0.8728 \\
\hline & 7 & 0.00309076 & 0.00034677 & \multicolumn{2}{|c|}{0.0259} & 0.8988 \\
\hline & 8 & 0.00274399 & 0.00019850 & \multicolumn{2}{|c|}{0.0230} & 0.9218 \\
\hline & 9 & 0.00254549 & 0.00033329 & \multicolumn{2}{|c|}{0.0214} & 0.9432 \\
\hline & 10 & 0.00221219 & 0.00099516 & \multicolumn{2}{|c|}{0.0186} & 0.9617 \\
\hline & 11 & 0.00121704 & 0.00029274 & \multicolumn{2}{|c|}{0.0102} & 0.9719 \\
\hline & 12 & 0.00092430 & 0.00008742 & \multicolumn{2}{|c|}{0.0078} & 0.9797 \\
\hline & 13 & 0.00083688 & 0.00018183 & \multicolumn{2}{|c|}{0.0070} & 0.9867 \\
\hline & 14 & 0.00065506 & 0.00010784 & & 055 & 0.9922 \\
\hline & 15 & 0.00054722 & 0.00016589 & & 046 & 0.9968 \\
\hline & 16 & 0.00038133 & & & 032 & 1.0000 \\
\hline Char & cter & Prin1 & Prin2 & Prin3 & Prin4 & Prin5 \\
\hline$x 1$ & ADS & 0.059554 & 0.073778 & 0.114126 & 0.172252 & 0.017451 \\
\hline$\times 2$ & MDS & 0.056526 & 0.090831 & 0.070339 & 0.128565 & 0.002427 \\
\hline$x 3$ & PDS & 0.026199 & 0.042645 & 0.013165 & 0.064528 & -.022006 \\
\hline$x 4$ & vs & 0.010585 & 0.053456 & -.053726 & 0.027104 & 0.087594 \\
\hline$\times 5$ & cs & 0.225342 & -.137602 & 0.945174 & -.046822 & 0.052229 \\
\hline$x 7$ & LSL & 0.003290 & 0.171011 & -.030633 & 0.490232 & 0.148993 \\
\hline$x 8$ & RSL & 0.002281 & 0.160345 & -.019106 & 0.500383 & 0.058889 \\
\hline$\times 9$ & LIL & 0.045774 & 0.155379 & 0.093954 & 0.381824 & -179974 \\
\hline$\times 10$ & RIL & 0.046523 & 0.143637 & 0.072615 & 0.425661 & -.245220 \\
\hline$x 12$ & LDB & 0.298078 & 0.291487 & -.030364 & -.109360 & 0.099476 \\
\hline$\times 13$ & RDB & 0.297422 & 0.272002 & -.024123 & -.078081 & 0.218001 \\
\hline$\times 14$ & LVB & 0.279952 & 0.300499 & -.056831 & -.114729 & 0.054696 \\
\hline$\times 15$ & RVB & 0.270491 & 0.266854 & -.047260 & -.113809 & 0.192059 \\
\hline$\times 17$ & $\mathrm{TE}$ & 0.284172 & 0.278998 & -.036991 & -.115425 & 0.174730 \\
\hline$\times 22$ & $\mathrm{BL}$ & -.658056 & 0.668241 & 0.243786 & -.224101 & -.088485 \\
\hline$\times 23$ & BS & -.307838 & -.134610 & 0.049426 & 0.145966 & 0.857296 \\
\hline
\end{tabular}




\begin{tabular}{|c|c|c|c|c|c|c|}
\hline & Prin6 & Prin7 & Prin8 & Pring & Prin10 & Prin11 \\
\hline$x 1$ & 0.125840 & 0.244873 & 0.596601 & 0.314434 & 0.049094 & -.612130 \\
\hline$x 2$ & 0.119105 & 0.179602 & 0.434525 & 0.241136 & 0.031916 & 0.786503 \\
\hline$x 3$ & 0.050740 & 0.080045 & 0.218626 & 0.144981 & 0.086302 & 0.059851 \\
\hline$x 4$ & 0.017121 & 0.020206 & 0.007123 & -.001598 & 0.000453 & -.008495 \\
\hline$\times 5$ & -.130327 & -.025352 & -.088518 & -.041914 & 0.002238 & 0.016413 \\
\hline$x 7$ & -.485459 & -.087746 & -.309213 & 0.570733 & -.202420 & -.001576 \\
\hline$x 8$ & -.430754 & 0.058196 & 0.257913 & -.668136 & 0.122298 & 0.010989 \\
\hline$\times 9$ & 0.509186 & 0.029818 & -.109797 & -188396 & -.689965 & -.014381 \\
\hline$\times 10$ & 0.409391 & -.188884 & -.281863 & 0.060374 & 0.663686 & -.018278 \\
\hline$x 12$ & 0.013132 & 0.423673 & -169187 & -.058804 & 0.049450 & -.024546 \\
\hline$x 13$ & 0.041450 & -.395102 & 0.150178 & -.022699 & -.018912 & 0.012324 \\
\hline$x 14$ & -.002155 & 0.479196 & -.252328 & -.033245 & 0.076233 & -.006433 \\
\hline$x 15$ & 0.064480 & -.526485 & 0.142240 & -.020334 & -.046351 & -.033726 \\
\hline$x 17$ & 0.031629 & -.008436 & -.038998 & 0.007143 & 0.011113 & -.001197 \\
\hline$x 22$ & -.039337 & -.027378 & 0.011984 & 0.003121 & 0.006352 & -.009508 \\
\hline \multirow[t]{2}{*}{$\times 23$} & 0.305671 & 0.097103 & -.095887 & -.058082 & 0.075698 & 0.009853 \\
\hline & Prin12 & \multicolumn{2}{|c|}{ Prin13 } & Prin14 & Prin15 & Prin16 \\
\hline$\times 1$ & 0.054706 & \multicolumn{2}{|c|}{-.191025} & -010339 & -.005130 & -.026104 \\
\hline$\times 2$ & 0.058686 & -.218564 & \multicolumn{2}{|c|}{-.002284} & -.051976 & -.012000 \\
\hline$x 3$ & -.120770 & \multicolumn{2}{|c|}{0.930853} & 0.132120 & 0.071358 & 0.014974 \\
\hline$x 4$ & 0.006597 & \multicolumn{2}{|c|}{-.027833} & 0.035446 & 0.030421 & 0.990913 \\
\hline$x 5$ & 0.017749 & 0.024770 & \multicolumn{2}{|c|}{0.020720} & 0.001326 & 0.056198 \\
\hline$x 7$ & -.018708 & -.003180 & \multicolumn{2}{|c|}{0.011037} & -.023221 & -.023749 \\
\hline$x 8$ & 0.045538 & 0.017685 & \multicolumn{2}{|c|}{-.009195} & -.007753 & -.024480 \\
\hline$\times 9$ & 0.000492 & 0.048271 & \multicolumn{2}{|c|}{-.002929} & 0.020274 & -.006205 \\
\hline$\times 10$ & -.005827 & -.049873 & \multicolumn{2}{|c|}{-.025597} & -.015288 & 0.004190 \\
\hline$x 12$ & -.539770 & -.017621 & -.0 & 7027 & -.536099 & -.011307 \\
\hline$x 13$ & -.530406 & -.119 & -.0 & 0227 & 0.551550 & -.046698 \\
\hline$\times 14$ & 0.346657 & -.047 & 0.4 & 1141 & 0.391882 & -.064919 \\
\hline$\times 15$ & 0.311441 & 0.032 & 0.4 & 0057 & -.489377 & -.025852 \\
\hline$\times 17$ & 0.431279 & 0.156 & -.7 & 0030 & 0.072785 & -.006007 \\
\hline$\times 22$ & -.015437 & -.001 & -.0 & 9432 & 0.004555 & -.000530 \\
\hline$\times 23$ & 0.008550 & 0.020 & 0.0 & 4428 & -.004433 & -.073726 \\
\hline
\end{tabular}




\section{APPENDIX VIII}

Table 8.1.-Ward's minimum variance cluster analysis using 10 principal components of males. Standard statistics and eigenvalues of the covariance matrix are listed. The rootmean-square total-sample standard deviation is 0.102072 .

\begin{tabular}{|c|c|c|c|c|c|}
\hline Variable & Mean & SD & Skewness & Kurtosis & Bimodality \\
\hline Print & 0 & 0.2291 & -0.3371 & 0.0338 & 0.3658 \\
\hline Prin2 & 0 & 0.1408 & -0.0897 & -0.1781 & 0.3559 \\
\hline Prin3 & 0 & 0.0984 & -0.1726 & 0.8552 & 0.2664 \\
\hline Prin4 & 0 & 0.0681 & -0.0804 & 0.1885 & 0.3146 \\
\hline Prin5 & 0 & 0.0654 & 0.1458 & 0.4460 & 0.2955 \\
\hline Prin6 & 0 & 0.0573 & 0.0667 & -0.1690 & 0.3535 \\
\hline Prin7 & 0 & 0.0544 & -0.0491 & 0.1216 & 0.3201 \\
\hline Prin8 & 0 & 0.0502 & 0.1605 & 0.4006 & 0.3007 \\
\hline Pring & 0 & 0.0497 & -0.0750 & -0.0735 & 0.3424 \\
\hline Prin10 & 0 & 0.0452 & 0.1654 & 0.2656 & 0.3136 \\
\hline Variable & Eigenvalue & \multicolumn{2}{|c|}{ Difference } & \multicolumn{2}{|c|}{ Cumulative } \\
\hline 1 & 0.05249525 & \multicolumn{2}{|l|}{0.03268153} & .5039 & 0.5039 \\
\hline 2 & 0.01981372 & \multicolumn{2}{|l|}{0.01012679} & .1902 & 0.6940 \\
\hline 3 & 0.00968692 & \multicolumn{2}{|l|}{0.00505428} & .0930 & 0.7870 \\
\hline 4 & 0.00463264 & \multicolumn{2}{|l|}{0.00036031} & .0445 & 0.8315 \\
\hline 5 & 0.00427234 & \multicolumn{2}{|c|}{0.00098836} & .0410 & 0.8725 \\
\hline 6 & 0.00328397 & \multicolumn{2}{|l|}{0.00031982} & 0.0315 & 0.9040 \\
\hline 7 & 0.00296416 & 0.0004398 & & 0.0285 & 0.9324 \\
\hline 8 & 0.00252431 & 0.0000538 & & 0.0242 & 0.9567 \\
\hline 9 & 0.00247047 & 0.00042666 & & 0.0237 & 0.9804 \\
\hline 10 & 0.00204381 & & & 0.0196 & 1.0000 \\
\hline
\end{tabular}


Table 8.2.-Ward's minimum variance cluster analysis using 10 principal components of females. Standard statistics and eigenvalues of the covariance matrix are listed. The root-mean-square total-sample standard deviation is 0.099827 .

\begin{tabular}{|c|c|c|c|c|c|c|}
\hline Variable & Mean & SD & \multicolumn{2}{|c|}{ Skewness } & Kurtosis & Bimodality \\
\hline Prin1 & 0 & 0.2202 & \multicolumn{2}{|c|}{-0.4567} & 0.00242 & 0.4009 \\
\hline Prin2 & 0 & 0.1335 & \multicolumn{2}{|c|}{-0.3793} & 0.3030 & 0.3451 \\
\hline Prin3 & 0 & 0.0973 & \multicolumn{2}{|c|}{0.0276} & -0.0234 & 0.3349 \\
\hline Prin4 & 0 & 0.0703 & \multicolumn{2}{|c|}{-0.1502} & 0.0797 & 0.3307 \\
\hline Prin5 & 0 & 0.0640 & \multicolumn{2}{|c|}{0.1517} & 0.1873 & 0.3197 \\
\hline Prin6 & 0 & 0.0620 & \multicolumn{2}{|c|}{0.1026} & 0.0283 & 0.3324 \\
\hline Print & 0 & 0.0570 & \multicolumn{2}{|c|}{-0.1001} & 0.0483 & 0.3300 \\
\hline Prin8 & 0 & 0.0541 & \multicolumn{2}{|c|}{-0.0211} & 0.1436 & 0.3170 \\
\hline Pring & 0 & 0.0520 & \multicolumn{2}{|c|}{0.1458} & 0.0456 & 0.3340 \\
\hline Prin10 & 0 & 0.0460 & \multicolumn{2}{|c|}{-0.1535} & 0.3358 & 0.3057 \\
\hline Variable & Eigenvalue & \multicolumn{3}{|c|}{ Difference } & \multicolumn{2}{|c|}{ Cumulative } \\
\hline 1 & 0.04847775 & \multicolumn{3}{|c|}{0.03064791} & .4865 & 0.4865 \\
\hline 2 & 0.01782984 & \multicolumn{2}{|c|}{0.00835933} & & .1789 & 0.6654 \\
\hline 3 & 0.00947051 & \multicolumn{2}{|c|}{0.00453300} & & .0950 & 0.7604 \\
\hline 4 & 0.00493751 & \multicolumn{2}{|c|}{0.00084396} & & .0495 & 0.8100 \\
\hline 5 & 0.00409355 & \multicolumn{2}{|c|}{0.00024584} & & .0411 & 0.8510 \\
\hline 6 & 0.00384771 & \multicolumn{2}{|c|}{0.00059848} & & .0386 & 0.8896 \\
\hline 7 & 0.00324923 & 0.0003 & & & .0326 & 0.9222 \\
\hline 8 & 0.00292887 & 0.0002 & & & .0294 & 0.9516 \\
\hline 9 & 0.00270078 & 0.0005 & & & .0271 & 0.9787 \\
\hline 10 & 0.00211873 & & & & .0213 & 1.0000 \\
\hline
\end{tabular}


Table 8.3.-Ward's minimum variance cluster analysis using 10 principal components of combined sexes. Standard statistics and eigenvalues of the covariance matrix are listed. The root-mean-square total-sample standard deviation is 0.107059 .

\begin{tabular}{|c|c|c|c|c|c|}
\hline Variable & Mean & SD & Skewness & Kurtosis & Bimodality \\
\hline Prin1 & 0 & 0.2320 & -0.3126 & 0.0255 & 0.3622 \\
\hline Prin2 & 0 & 0.1394 & -0.1567 & -0.1809 & 0.3627 \\
\hline Prin3 & 0 & 0.1129 & -0.1629 & -0.5244 & 0.4137 \\
\hline Prin4 & 0 & 0.0981 & -0.0292 & 0.4004 & 0.2939 \\
\hline Prin5 & 0 & 0.0665 & -0.0825 & 0.3140 & 0.3033 \\
\hline Prin6 & 0 & 0.0632 & 0.0914 & 0.2341 & 0.3113 \\
\hline Prin7 & 0 & 0.0556 & -0.0796 & 0.1257 & 0.3214 \\
\hline Prin8 & 0 & 0.0524 & -0.0517 & 0.0249 & 0.3309 \\
\hline Pring & 0 & 0.0505 & 0.0444 & 0.0591 & 0.3269 \\
\hline Prin10 & 0 & 0.0470 & 0.0673 & 0.2347 & 0.3100 \\
\hline Variable & Eigenvalue & \multicolumn{2}{|l|}{ Difference } & \multicolumn{2}{|c|}{ Cumulative } \\
\hline 1 & 0.05381335 & \multicolumn{2}{|l|}{0.03439440} & .4695 & 0.4695 \\
\hline 2 & 0.01941895 & \multicolumn{2}{|l|}{0.00667414} & .1694 & 0.6389 \\
\hline 3 & 0.01274480 & \multicolumn{2}{|l|}{0.00311316} & 1112 & 0.7501 \\
\hline 4 & 0.00963165 & \multicolumn{2}{|l|}{0.00520821} & .0840 & 0.8342 \\
\hline 5 & 0.00442344 & \multicolumn{2}{|l|}{0.00043264} & .0386 & 0.8728 \\
\hline 6 & 0.00399080 & \multicolumn{2}{|l|}{0.00090004} & .0348 & 0.9076 \\
\hline 7 & 0.00309076 & \multicolumn{2}{|l|}{0.00034677} & .0270 & 0.9345 \\
\hline 8 & 0.00274399 & \multicolumn{2}{|l|}{0.00019850} & .0239 & 0.9585 \\
\hline 9 & 0.00254549 & \multicolumn{2}{|l|}{0.00033329} & .0222 & 0.9807 \\
\hline 10 & 0.00221219 & \multicolumn{3}{|c|}{0.0193} & 1.0000 \\
\hline
\end{tabular}




\section{APPENDIX IX}

Table 9.1.-Canonical discriminant analysis of the five-cluster model for males.

Multivariate statistics, $F$ approximations, canonical correlations, eigenvalues, and the total variation explained by each canonical variable are listed.

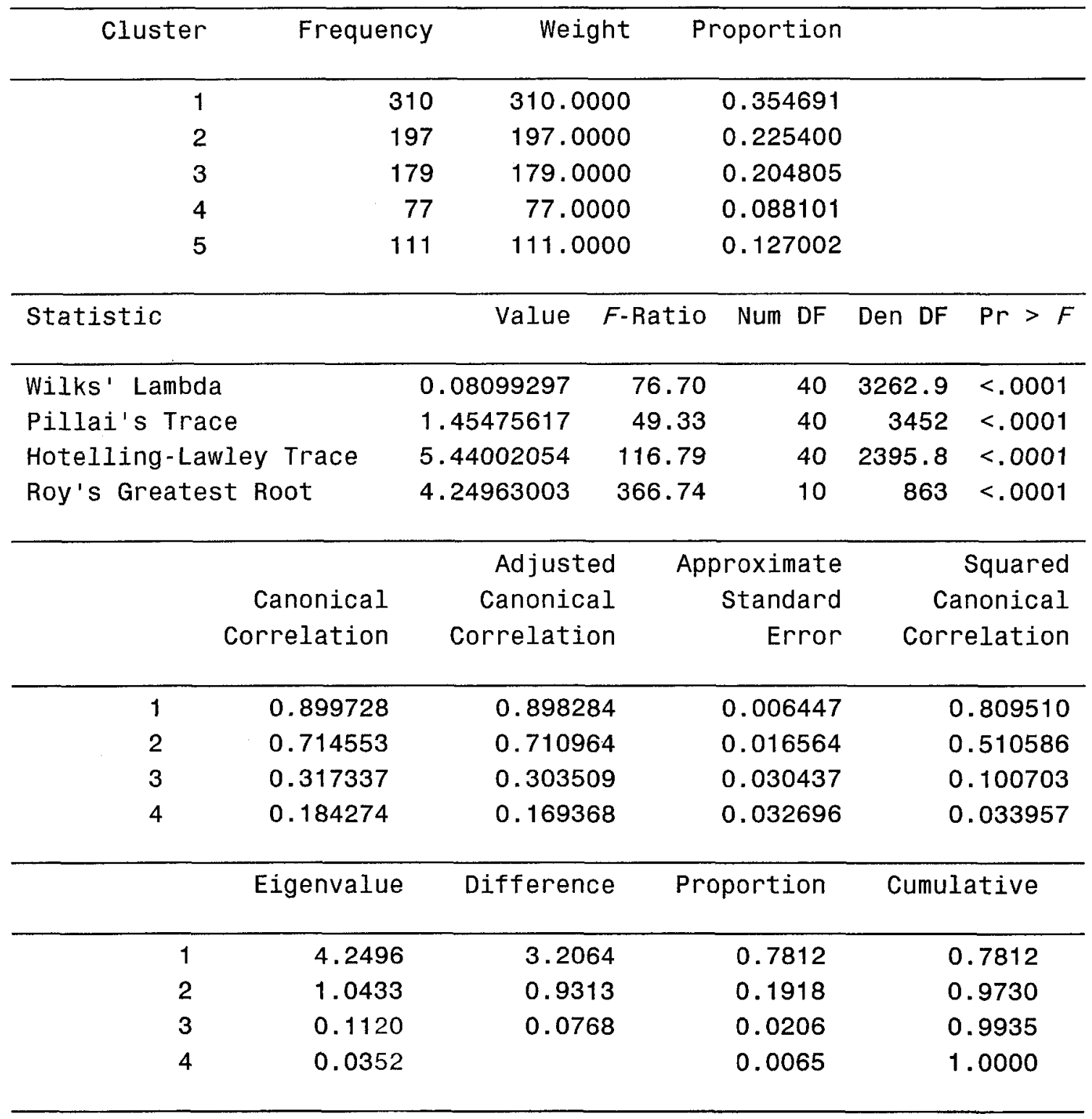


Table 9.2.-Canonical discriminant analysis of the five-cluster model for females. Multivariate statistics, $F$ approximations, canonical correlations, eigenvalues, and the total variation explained by each canonical variable are listed..

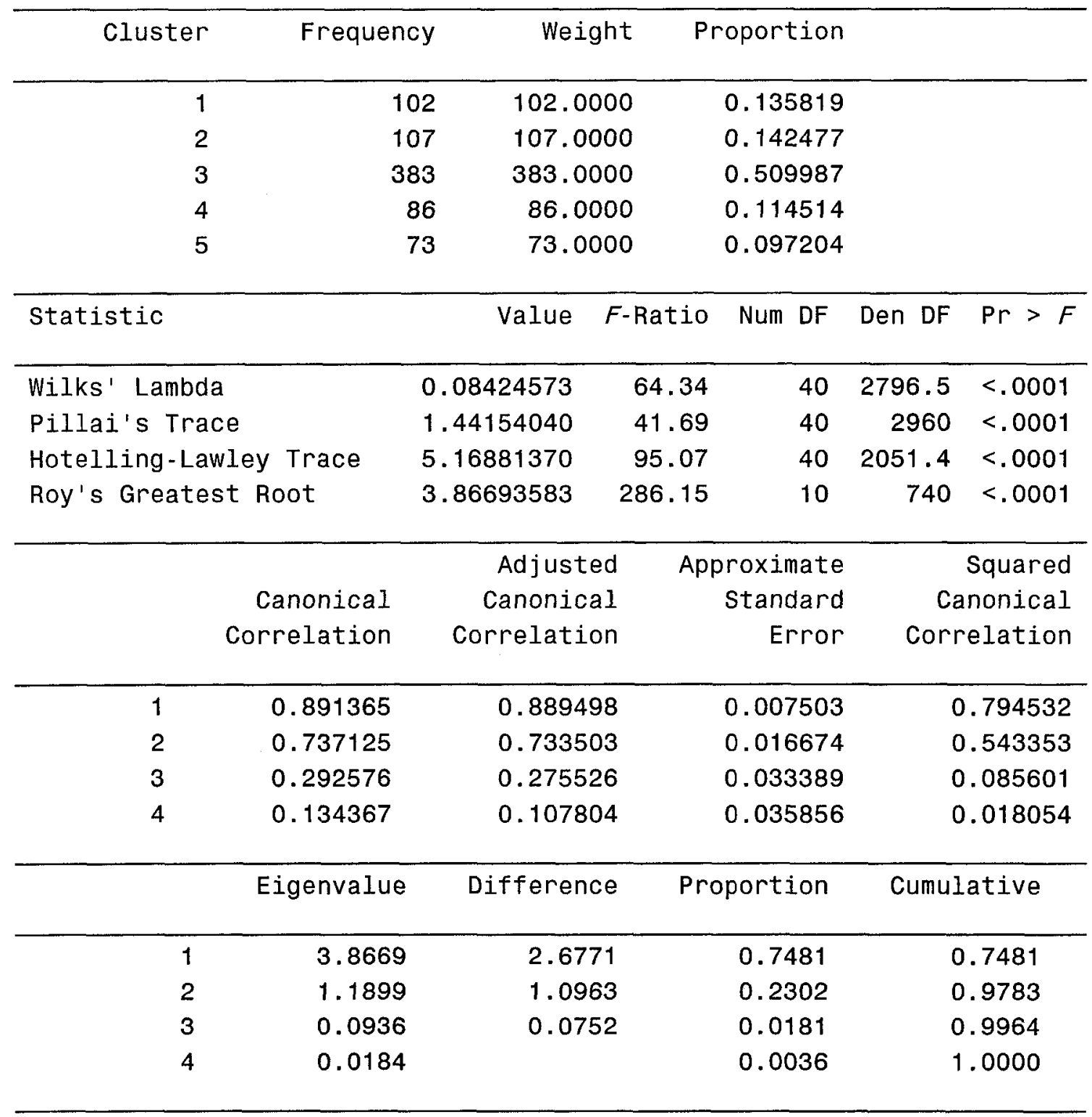


Table 9.3.-Canonical discriminant analysis of the five-cluster model for combined sexes. Multivariate statistics, $F$ approximations, canonical correlations, eigenvalues, and the total variation explained by each canonical variable are listed..

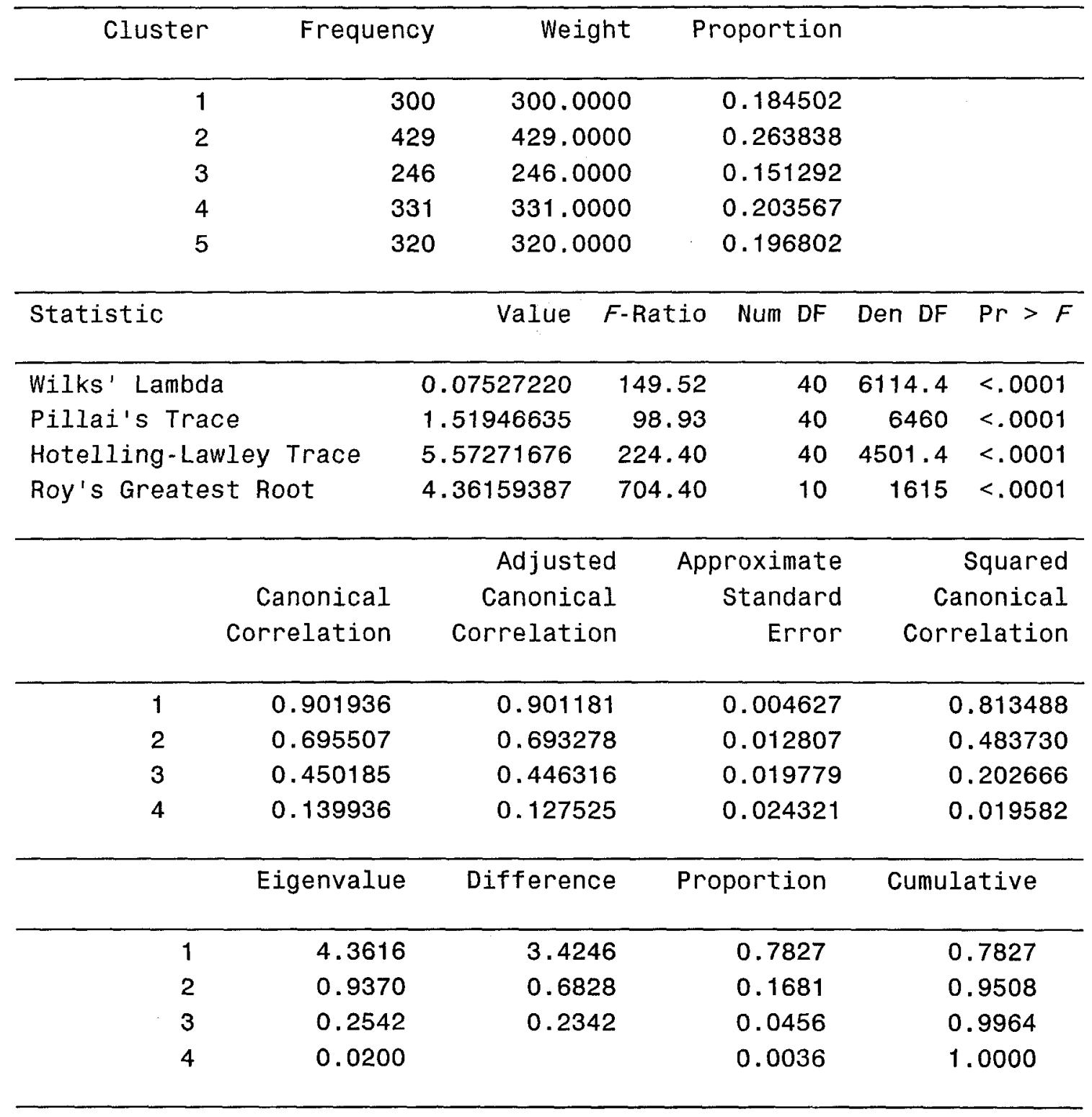




\section{APPENDIX X}

\section{DISCRIMINANT FUNCTION ANALYSES OF CLUSTER MODELS}

Table 10.1. - Discriminant function analysis of the two-cluster model for males $(\mathrm{n}=874)$.

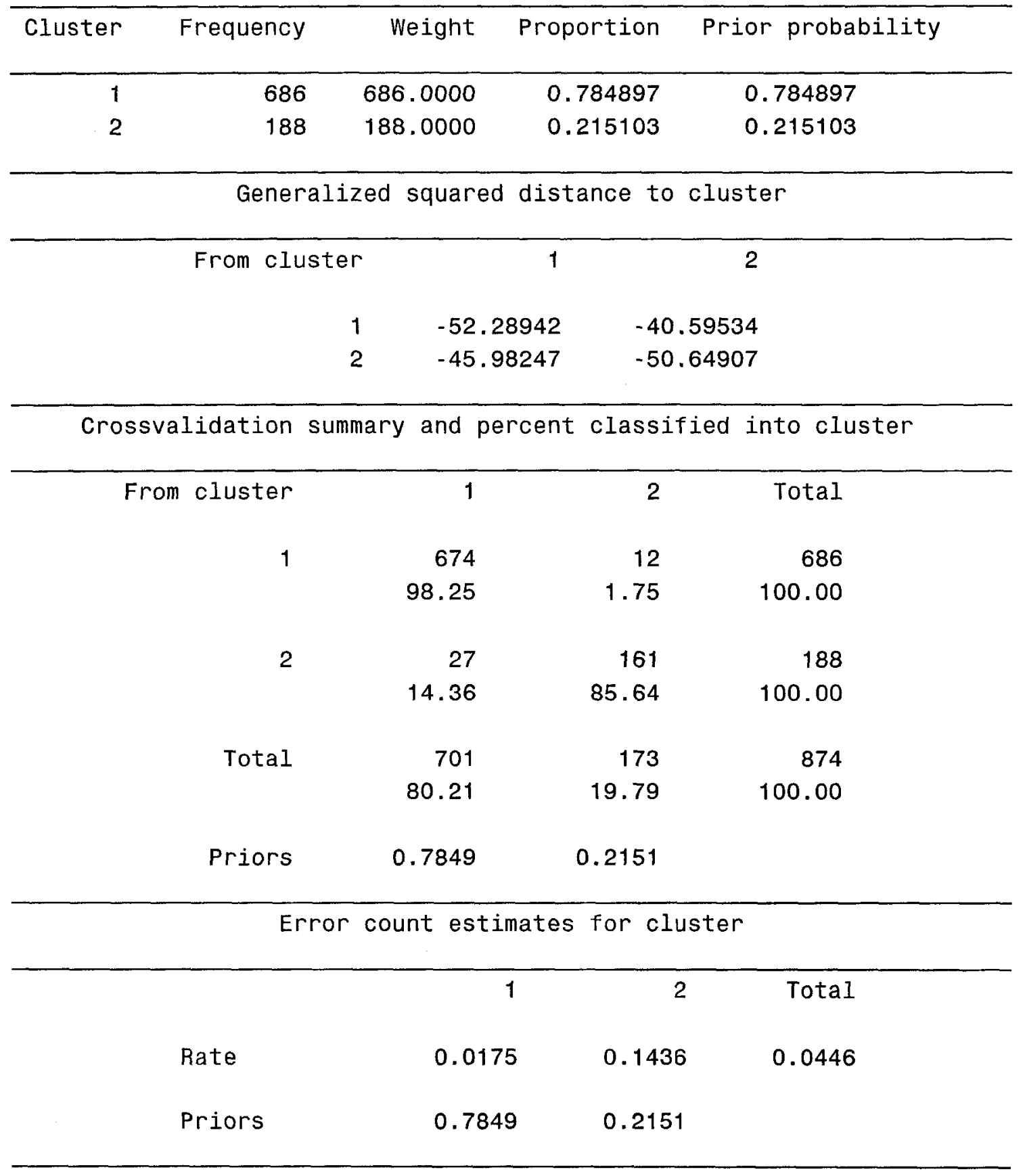


Table 10.2.-Discriminant function analysis of the two-cluster model for females $(\mathrm{n}=751)$.

\begin{tabular}{|c|c|c|c|c|}
\hline Cluster & Frequency & Weight & Proportion & Prior probability \\
\hline 1 & 295 & 295.0000 & 0.392810 & 0.392810 \\
\hline 2 & 456 & 456.0000 & 0.607190 & 0.607190 \\
\hline \multicolumn{5}{|c|}{ Generalized squared distance to cluster } \\
\hline \multicolumn{3}{|c|}{ From cluster } & \multicolumn{2}{|r|}{2} \\
\hline & & \multicolumn{2}{|c|}{-50.51243} & 42.83205 \\
\hline & & \multicolumn{2}{|c|}{-44.39331} & 52.26720 \\
\hline \multicolumn{5}{|c|}{ Crossvalidation summary and percent classified into cluster } \\
\hline \multicolumn{2}{|c|}{ From cluster } & 1 & 2 & Total \\
\hline \multirow{2}{*}{\multicolumn{2}{|c|}{1}} & 266 & 29 & 295 \\
\hline & & 90.17 & 9.83 & 100.00 \\
\hline \multirow{2}{*}{\multicolumn{2}{|c|}{2}} & 9 & 447 & 456 \\
\hline & & 1.97 & 98.03 & 100.00 \\
\hline \multirow{2}{*}{\multicolumn{2}{|c|}{ Total }} & 275 & 476 & 751 \\
\hline & & 36.62 & 63.38 & 100.00 \\
\hline \multicolumn{2}{|r|}{ Priors } & 0.39281 & 0.60719 & \\
\hline \multicolumn{5}{|c|}{ Error count estimates for cluster } \\
\hline & & \multicolumn{2}{|c|}{1} & Total \\
\hline \multicolumn{2}{|r|}{ Rate } & 0.0 & 0.019 & 0.0506 \\
\hline \multicolumn{2}{|r|}{ Priors } & 0.3 & 0.607 & \\
\hline
\end{tabular}


Table 10.3.-Discriminant function analysis of the two-cluster model for combined sexes $(\mathrm{n}=1626)$.

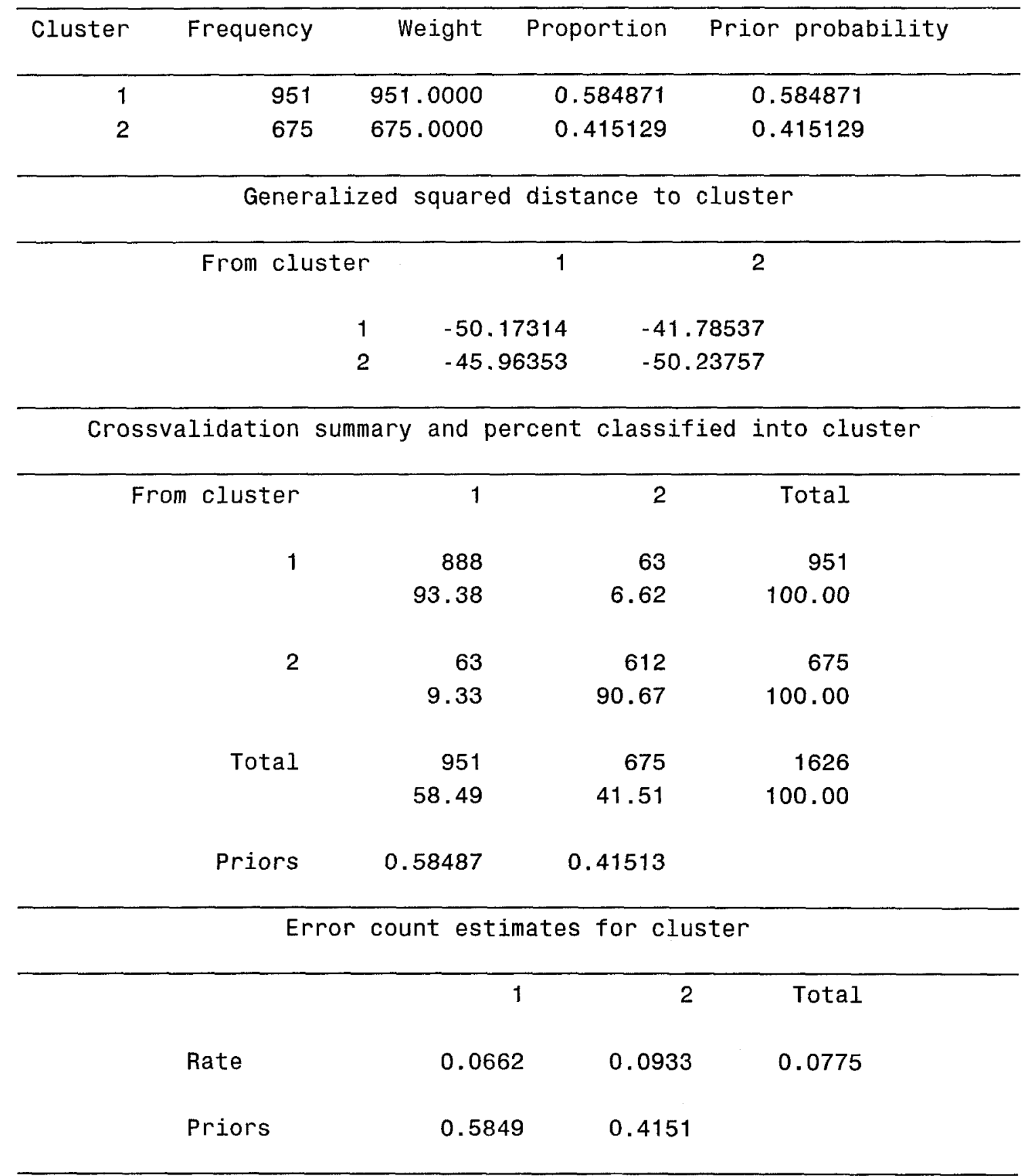


Table 10.4.--Discriminant function analysis of the three-cluster model for males $(\mathrm{n}=874)$.

\begin{tabular}{|c|c|c|c|c|}
\hline Cluster & Frequency & Weight & Proportion & Prior probability \\
\hline 1 & 507 & 507.0000 & 0.580092 & 0.580092 \\
\hline 2 & 179 & 179.0000 & 0.204805 & 0.204805 \\
\hline 3 & 188 & 188.0000 & 0.215103 & 0.215103 \\
\hline \multicolumn{5}{|c|}{ Generalized squared distance to cluster } \\
\hline From & cluster & 1 & 2 & 3 \\
\hline & 1 & -53.28001 & -34.19357 & -43.18824 \\
\hline & 2 & -39.31850 & -50.62130 & -29.72275 \\
\hline & 3 & -44.38387 & -1.62528 & -50.64907 \\
\hline
\end{tabular}

\begin{tabular}{|c|c|c|c|c|}
\hline Crossval: & on summary a & d percent $\mathrm{cl}$ & sified in & luster \\
\hline From cluster & 1 & 2 & 3 & Total \\
\hline \multirow[t]{2}{*}{1} & 482 & 11 & 14 & 507 \\
\hline & 95.07 & 2.17 & 2.76 & 100.00 \\
\hline \multirow[t]{2}{*}{2} & 8 & 167 & 4 & 179 \\
\hline & 4.47 & 93.30 & 2.23 & 100.00 \\
\hline \multirow[t]{2}{*}{3} & 23 & 0 & 165 & 188 \\
\hline & 12.23 & 0.00 & 87.77 & 100.00 \\
\hline \multirow[t]{2}{*}{ Total } & 513 & 178 & 183 & 874 \\
\hline & 58.70 & 20.37 & 20.94 & 100.00 \\
\hline \multirow[t]{3}{*}{ Priors } & 0.58009 & 0.20481 & 0.2151 & \\
\hline & \multicolumn{4}{|c|}{ Error count estimates for cluster } \\
\hline & 1 & 2 & 3 & Total \\
\hline Rate & 0.0493 & 0.0670 & 0.1223 & 0.0686 \\
\hline Priors & 0.5801 & 0.2048 & 0.2151 & \\
\hline
\end{tabular}


Table 10.5.-Discriminant function analysis of the three-cluster model for females $(\mathrm{n}=751)$.

\begin{tabular}{|c|c|c|c|c|}
\hline Cluster & Frequency & Weight & Proportion & Prior probability \\
\hline 1 & 295 & 295.0000 & 0.392810 & 0.392810 \\
\hline 2 & 383 & 383.0000 & 0.509987 & 0.509987 \\
\hline 3 & 73 & 73.0000 & 0.097204 & 0.097204 \\
\hline \multicolumn{5}{|c|}{ Generalized squared distance to cluster } \\
\hline From & cluster & 1 & 2 & 3 \\
\hline & 1 & -50.51243 & -41.13907 & -27.21234 \\
\hline & 2 & -44.74978 & -53.15779 & -37.79256 \\
\hline & 3 & -37.47845 & -39.65821 & -49.44963 \\
\hline
\end{tabular}

Crossvalidation summary and percent classified into cluster

\begin{tabular}{|c|c|c|c|c|}
\hline From cluster & 1 & 2 & 3 & Total \\
\hline \multirow[t]{2}{*}{1} & 271 & 24 & 0 & 295 \\
\hline & 91.86 & 8.14 & 0.00 & 100.00 \\
\hline \multirow[t]{2}{*}{2} & 14 & 365 & 4 & 383 \\
\hline & 3.66 & 95.30 & 1.04 & 100.00 \\
\hline \multirow[t]{2}{*}{3} & 4 & 10 & 59 & 73 \\
\hline & 5.48 & 13.70 & 80.82 & 100.00 \\
\hline \multirow[t]{2}{*}{ Total } & 289 & 399 & 63 & 751 \\
\hline & 38.48 & 53.13 & 8.39 & 100.00 \\
\hline \multirow[t]{3}{*}{ Priors } & 0.39281 & 0.50999 & 0.0972 & \\
\hline & \multicolumn{4}{|c|}{ Error count estimates for cluster } \\
\hline & 1 & 2 & 3 & Total \\
\hline Rate & 0.0814 & 0.0470 & 0.1918 & 0.0746 \\
\hline Priors & 0.3928 & 0.5100 & 0.0972 & \\
\hline
\end{tabular}


Table 10.6.-Discriminant function analysis of the three-cluster model for combined sexes $(n=1626)$.

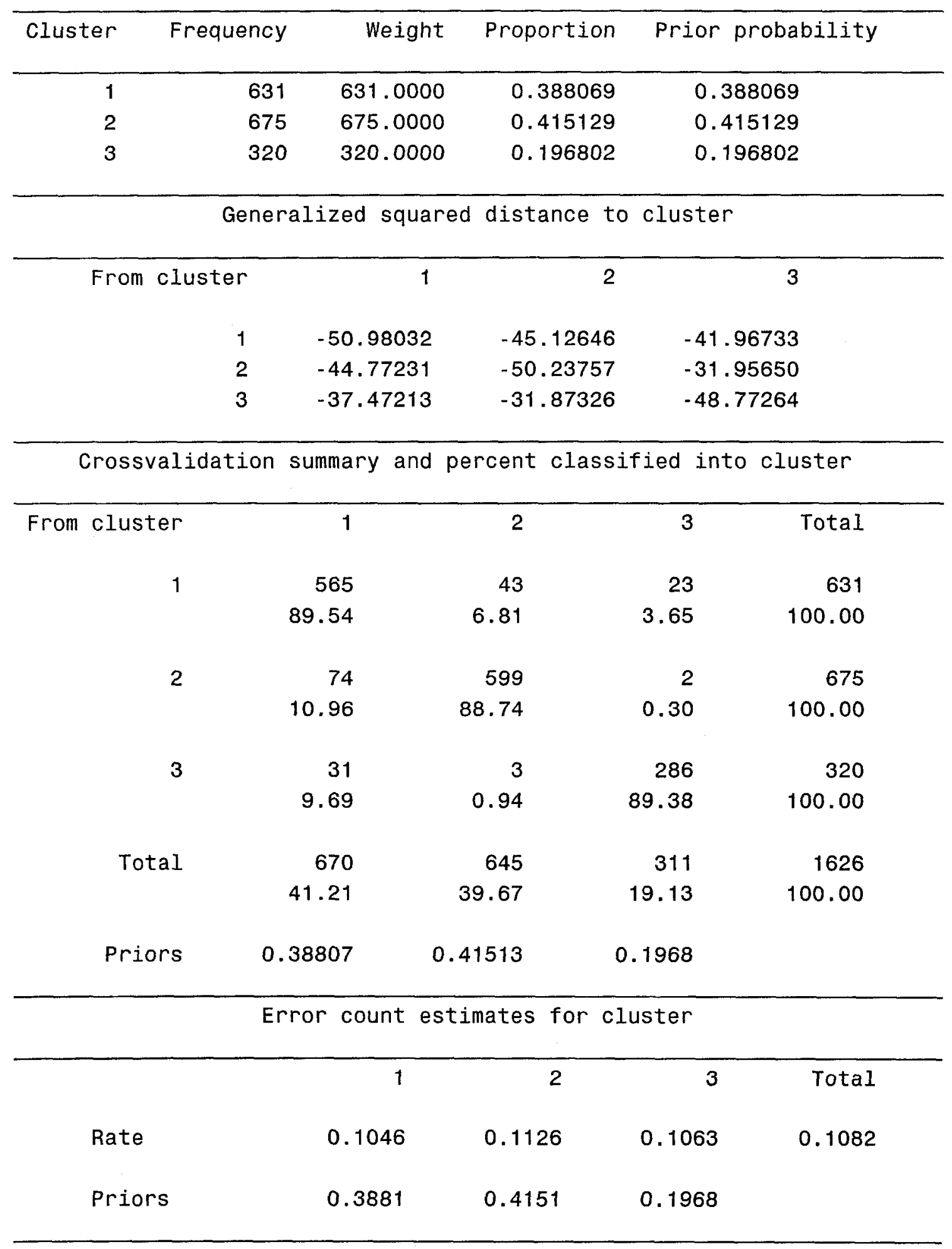


Table 10.7.-Discriminant function analysis of the four-cluster model for males $(\mathrm{n}=874)$.

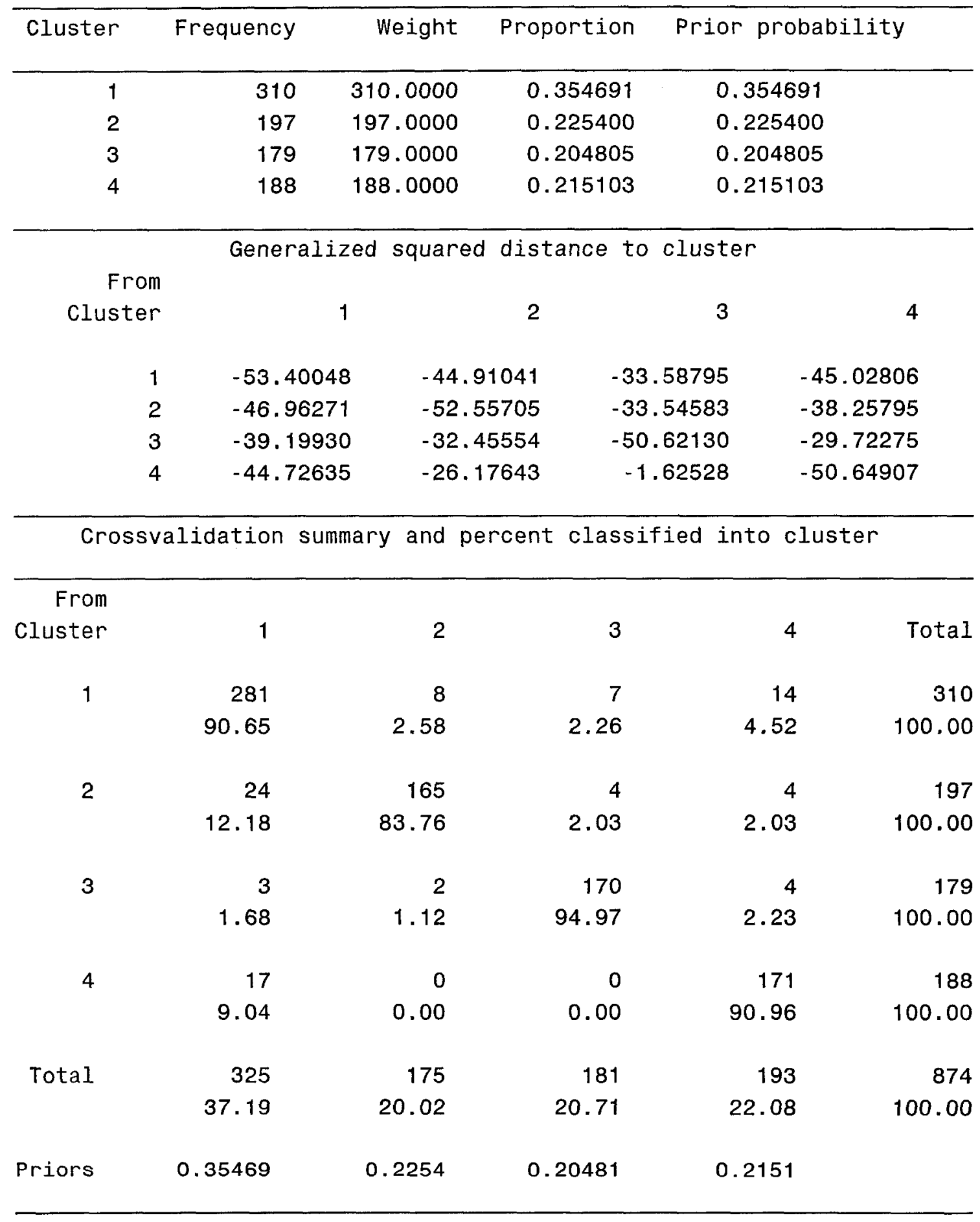




\begin{tabular}{lrccrr}
\hline \multicolumn{5}{c}{ Error count estimates for cluster } \\
& 1 & 2 & 3 & 4 & Total \\
Rate & 0.0935 & 0.1624 & 0.0503 & 0.0904 & 0.0995 \\
Priors & 0.3547 & 0.2254 & 0.2048 & 0.2151 & \\
\hline
\end{tabular}


Table 10.8.-Discriminant function analysis of the four-cluster model for females $(\mathrm{n}=751)$.

\begin{tabular}{|c|c|c|c|c|c|}
\hline Cluster & Frequency & Weight & Proportion & \multicolumn{2}{|c|}{ Prior probability } \\
\hline 1 & 102 & 102.0000 & 0.135819 & \multicolumn{2}{|c|}{0.135819} \\
\hline 2 & 193 & 193.0000 & 0.256991 & \multicolumn{2}{|c|}{0.256991} \\
\hline 3 & 383 & 383.0000 & 0.509987 & \multicolumn{2}{|c|}{0.509987} \\
\hline 4 & 73 & 73.0000 & 0.097204 & \multicolumn{2}{|c|}{0.097204} \\
\hline \multicolumn{6}{|c|}{ Generalized squared distance to cluster } \\
\hline \multicolumn{6}{|c|}{ 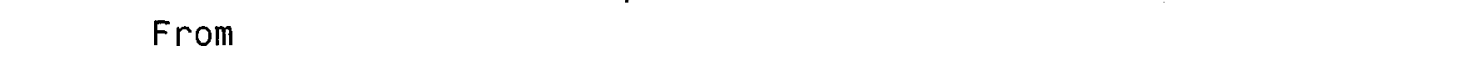 } \\
\hline \multicolumn{2}{|c|}{ Cluster } & 1 & 2 & 3 & 4 \\
\hline & \multicolumn{2}{|c|}{-51.93489} & \multicolumn{2}{|c|}{-46.38189} & -36.89116 \\
\hline & -32.73930 & \multicolumn{2}{|c|}{-50.22374} & -34.07372 & -19.99389 \\
\hline & -37.44338 & \multicolumn{2}{|c|}{-39.64289} & -53.15779 & -37.79256 \\
\hline & \multicolumn{2}{|c|}{-19.03394} & 2188 & -39.65821 & -49.44963 \\
\hline \multicolumn{3}{|c|}{ Crossvalidation summ } & rcent classi & \multicolumn{2}{|c|}{ cluster } \\
\hline From & & & & & \\
\hline Cluster & 1 & 2 & 3 & 4 & Total \\
\hline 1 & 86 & 6 & 9 & 1 & 102 \\
\hline & 84.31 & 5.88 & 8.82 & 0.98 & 100.00 \\
\hline 2 & 1 & 186 & 6 & 0 & 193 \\
\hline & 0.52 & 96.37 & 3.11 & 0.00 & 100.00 \\
\hline 3 & 7 & 6 & 366 & 4 & 383 \\
\hline & 1.83 & 1.57 & 95.56 & 1.04 & 100.00 \\
\hline 4 & 2 & 0 & 12 & 59 & 73 \\
\hline & 2.74 & 0.00 & 16.44 & 80.82 & 100.00 \\
\hline Total & 96 & 198 & 393 & 64 & 751 \\
\hline & 12.78 & 26.36 & 52.33 & 8.52 & 100.00 \\
\hline Priors & 0.13582 & 0.25699 & 0.50999 & 0.0972 & \\
\hline
\end{tabular}




\begin{tabular}{lrccrr}
\hline \multicolumn{5}{c}{ Error count estimates for cluster } \\
& 1 & 2 & 3 & 4 & Total \\
Rate & 0.1569 & 0.0363 & 0.0444 & 0.1918 & 0.0719 \\
Priors & 0.1358 & 0.2570 & 0.5100 & 0.0972 & \\
\hline
\end{tabular}


Table 10.9.-Discriminant function analysis of the four-cluster model for combined $\operatorname{sexes}(n=1626)$.

\begin{tabular}{|c|c|c|c|c|c|}
\hline Cluster & Frequency & Weight & Proportion & \multicolumn{2}{|c|}{ Prior probability } \\
\hline 1 & 631 & 631.0000 & 0.388069 & \multicolumn{2}{|c|}{0.388069} \\
\hline 2 & 429 & 429.0000 & 0.263838 & \multicolumn{2}{|c|}{0.263838} \\
\hline 3 & 246 & 246.0000 & 0.151292 & \multicolumn{2}{|c|}{0.151292} \\
\hline 4 & 320 & 320.0000 & 0.196802 & \multicolumn{2}{|c|}{0.196802} \\
\hline \multicolumn{6}{|c|}{ Generalized squared distance to cluster } \\
\hline \multicolumn{6}{|c|}{ 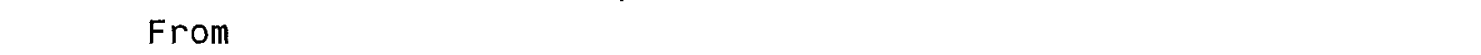 } \\
\hline \multicolumn{2}{|c|}{ Cluster } & 1 & 2 & 3 & 4 \\
\hline & \multicolumn{2}{|c|}{-50.98032} & 0461 & -34.41304 & -41.96733 \\
\hline & \multicolumn{2}{|c|}{-45.99323} & 8594 & -34.77510 & -36.87971 \\
\hline & \multicolumn{2}{|c|}{-32.59789} & 1580 & -48.83783 & -17.82174 \\
\hline & \multicolumn{2}{|c|}{-37.47213} & 1010 & 1.88766 & -48.77264 \\
\hline \multicolumn{6}{|c|}{ Crossvalidation summary and percent classified into cluster } \\
\hline \multicolumn{6}{|l|}{ From } \\
\hline Cluster & 1 & 2 & 3 & 4 & Total \\
\hline \multirow[t]{2}{*}{1} & 574 & 23 & 10 & 24 & 631 \\
\hline & 90.97 & 3.65 & 1.58 & 3.80 & 100.00 \\
\hline \multirow[t]{2}{*}{2} & 40 & 379 & 6 & 4 & 429 \\
\hline & 9.32 & 88.34 & 1.40 & 0.93 & 100.00 \\
\hline \multirow[t]{2}{*}{3} & 13 & 4 & 229 & 0 & 246 \\
\hline & 5.28 & 1.63 & 93.09 & 0.00 & 100.00 \\
\hline \multirow[t]{2}{*}{4} & 31 & 1 & 0 & 288 & 320 \\
\hline & 9.69 & 0.31 & 0.00 & 90.00 & 100.00 \\
\hline \multirow[t]{2}{*}{ Total } & 658 & 407 & 245 & 316 & 1626 \\
\hline & 40.47 & 25.03 & 15.07 & 19.43 & 100.00 \\
\hline Priors & 0.38807 & 0.26384 & 0.15129 & 0.1968 & \\
\hline
\end{tabular}




\begin{tabular}{lrrrrr}
\hline \multicolumn{5}{c}{ Error count estimates for cluster } \\
& 1 & 2 & 3 & 4 & Total \\
Rate & 0.0903 & 0.1166 & 0.0691 & 0.1000 & 0.0959 \\
Priors & 0.3881 & 0.2638 & 0.1513 & 0.1968 & \\
\hline
\end{tabular}


Table 10.10.-Discriminant function analysis of the five-cluster model for males $(\mathrm{n}=874)$.

\begin{tabular}{|c|c|c|c|c|c|}
\hline Cluster & Frequency & Weight & Proportion & Prior probab & ility \\
\hline 1 & 310 & 310.0000 & 0.354691 & 0.354691 & \\
\hline 2 & 197 & 197.0000 & 0.225400 & 0.225400 & \\
\hline 3 & 179 & 179.0000 & 0.204805 & 0.204805 & \\
\hline 4 & 77 & 77.0000 & 0.088101 & 0.088101 & \\
\hline 5 & 111 & 111.0000 & 0.127002 & 0.127002 & \\
\hline \multicolumn{6}{|c|}{ Generalized squared distance to cluster } \\
\hline Cluster & 1 & 2 & 3 & 4 & 5 \\
\hline 1 & -53.40048 & -44.91041 & -33.58795 & -39.13878 & -42.34088 \\
\hline 2 & -46.96271 & -52.55705 & -33.54583 & -20.78394 & -38.52823 \\
\hline 3 & -39.19930 & -32.45554 & -50.62130 & -1.69434 & -23.47282 \\
\hline 4 & -45.30724 & -26.99143 & -24.61328 & -52.44713 & -43.99114 \\
\hline 5 & -38.45357 & -18.93472 & 20.44321 & -33.66862 & -49.87341 \\
\hline
\end{tabular}

Crossvalidation summary and percent classified into cluster

\begin{tabular}{|c|c|c|c|c|c|c|}
\hline Cluster & 1 & 2 & 3 & 4 & 5 & Total \\
\hline \multirow[t]{2}{*}{1} & 285 & 8 & 7 & 3 & 7 & 310 \\
\hline & 91.94 & 2.58 & 2.26 & 0.97 & 2.26 & 100.00 \\
\hline \multirow[t]{2}{*}{2} & 24 & 166 & 4 & 0 & 3 & 197 \\
\hline & 12.18 & 84.26 & 2.03 & 0.00 & 1.52 & 100.00 \\
\hline \multirow[t]{2}{*}{3} & 3 & 2 & 172 & 2 & 0 & 179 \\
\hline & 1.68 & 1.12 & 96.09 & 1.12 & 0.00 & 100.00 \\
\hline \multirow[t]{2}{*}{4} & 13 & 0 & 2 & 57 & 5 & 77 \\
\hline & 16.88 & 0.00 & 2.60 & 74.03 & 6.49 & 100.00 \\
\hline \multirow[t]{2}{*}{5} & 8 & 0 & 0 & 3 & 100 & 111 \\
\hline & 7.21 & 0.00 & 0.00 & 2.70 & 90.09 & 100.00 \\
\hline \multirow[t]{2}{*}{ Total } & 333 & 176 & 185 & 65 & 115 & 874 \\
\hline & 38.10 & 20.14 & 21.17 & 7.44 & 13.16 & 100.00 \\
\hline Priors & 0.35469 & 0.2254 & 0.20481 & 0.0881 & 0.127 & \\
\hline
\end{tabular}




\begin{tabular}{lrrrrrr}
\hline \multicolumn{7}{c}{ Error count estimates for cluster } \\
& 1 & 2 & 3 & 4 & 5 & Total \\
Rate & 0.0806 & 0.1574 & 0.0391 & 0.2597 & 0.0991 & 0.1076 \\
Priors & 0.3547 & 0.2254 & 0.2048 & 0.0881 & 0.1270 & \\
\hline
\end{tabular}


Table 10.11. - Discriminant function analysis of the five-cluster model for females $(\mathrm{n}=751)$.

\begin{tabular}{rrrrc}
\hline Cluster & Frequency & Weight & Proportion & Prior probability \\
& & & & \\
2 & 102 & 102.0000 & 0.135819 & 0.135819 \\
3 & 107 & 107.0000 & 0.142477 & 0.142477 \\
4 & 383 & 383.0000 & 0.509987 & 0.509987 \\
5 & 86 & 86.0000 & 0.114514 & 0.114514 \\
& 73 & 73.0000 & 0.097204 & 0.097204 \\
\hline
\end{tabular}

Generalized squared distance to cluster

\begin{tabular}{rrrrrr}
\hline Cluster & 1 & 2 & 3 & 4 & 5 \\
1 & -51.93489 & -37.25112 & -46.38189 & -39.85819 & -36.89116 \\
2 & -33.56764 & -50.82394 & -38.82308 & -43.94194 & -20.68307 \\
3 & -37.44338 & -38.70204 & -53.15779 & -26.51494 & -37.79256 \\
4 & -25.05259 & -36.61439 & -25.12099 & -49.86093 & -15.94964 \\
5 & -19.03394 & -12.29688 & -39.65821 & -5.02228 & -49.44963
\end{tabular}

Crossvalidation summary and percent classified into cluster

\begin{tabular}{|c|c|c|c|c|c|c|}
\hline Cluster & 1 & 2 & 3 & 4 & 5 & Total \\
\hline \multirow[t]{2}{*}{1} & 86 & 2 & 9 & 4 & 1 & 102 \\
\hline & 84.31 & 1.96 & 8.82 & 3.92 & 0.98 & 100.00 \\
\hline \multirow[t]{2}{*}{2} & 1 & 91 & 6 & 9 & 0 & 107 \\
\hline & 0.93 & 85.05 & 5.61 & 8.41 & 0.00 & 100.00 \\
\hline \multirow[t]{2}{*}{3} & 7 & 3 & 369 & 0 & 4 & 383 \\
\hline & 1.83 & 0.78 & 96.34 & 0.00 & 1.04 & 100.00 \\
\hline \multirow[t]{2}{*}{4} & 2 & 11 & 0 & 73 & 0 & 86 \\
\hline & 2.33 & 12.79 & 0.00 & 84.88 & 0.00 & 100.00 \\
\hline \multirow[t]{2}{*}{5} & 2 & 0 & 12 & 0 & 59 & 73 \\
\hline & 2.74 & 0.00 & 16.44 & 0.00 & 80.82 & 100.00 \\
\hline \multirow[t]{2}{*}{ Total } & 98 & 107 & 396 & 86 & 64 & 751 \\
\hline & 13.05 & 14.25 & 52.73 & 11.45 & 8.52 & 100.00 \\
\hline Priors & 0.13582 & 0.14248 & 0.50999 & 0.11451 & 0.0972 & \\
\hline
\end{tabular}




\begin{tabular}{lrrrrrr}
\hline \multicolumn{6}{c}{ Error count estimates for cluster } \\
& 1 & 2 & 3 & 4 & 5 & Total \\
Rate & 0.1569 & 0.1495 & 0.0366 & 0.1512 & 0.1918 & 0.0972 \\
Priors & 0.1358 & 0.1425 & 0.5100 & 0.1145 & 0.0972 & \\
\hline
\end{tabular}


Table 10.12.-Discriminant function analysis of the five-cluster model for combined sexes $(n=1626)$.

\begin{tabular}{rrrrr}
\hline Cluster & Frequency & Weight & Proportion & Prior probability \\
\hline 1 & 300 & 300.0000 & 0.184502 & 0.184502 \\
2 & 429 & 429.0000 & 0.263838 & 0.263838 \\
3 & 246 & 246.0000 & 0.151292 & 0.151292 \\
4 & 331 & 331.0000 & 0.203567 & 0.203567 \\
5 & 320 & 320.0000 & 0.196802 & 0.196802 \\
\hline
\end{tabular}

Generalized squared distance to cluster

\begin{tabular}{rrrrrr}
\hline Cluster & 1 & 2 & 3 & 4 & 5 \\
& & & & & \\
1 & -50.89987 & -38.99122 & -34.32500 & -42.99605 & -40.37980 \\
2 & -39.91639 & -51.98594 & -34.77510 & -41.68473 & -36.87971 \\
3 & -32.51111 & -31.51580 & -48.83783 & -24.19270 & -17.82174 \\
4 & -43.47702 & -45.97727 & -32.29146 & -51.12363 & -41.92210 \\
5 & -34.71687 & -22.61010 & 1.88766 & -29.85226 & -48.77264
\end{tabular}

Crossvalidation summary and percent classified into cluster

\begin{tabular}{|c|c|c|c|c|c|c|}
\hline Cluster & 1 & 2 & 3 & 4 & 5 & Total \\
\hline \multirow[t]{2}{*}{1} & 252 & 11 & 8 & 10 & 19 & 300 \\
\hline & 84.00 & 3.67 & 2.67 & 3.33 & 6.33 & 100.00 \\
\hline \multirow[t]{2}{*}{2} & 8 & 394 & 6 & 16 & 5 & 429 \\
\hline & 1.86 & 91.84 & 1.40 & 3.73 & 1.17 & 100.00 \\
\hline \multirow[t]{2}{*}{3} & 4 & 6 & 229 & 7 & 0 & 246 \\
\hline & 1.63 & 2.44 & 93.09 & 2.85 & 0.00 & 100.00 \\
\hline \multirow[t]{2}{*}{4} & 14 & 15 & 2 & 286 & 14 & 331 \\
\hline & 4.23 & 4.53 & 0.60 & 86.40 & 4.23 & 100.00 \\
\hline \multirow[t]{2}{*}{5} & 11 & 1 & 0 & 12 & 296 & 320 \\
\hline & 3.44 & 0.31 & 0.00 & 3.75 & 92.50 & 100.00 \\
\hline \multirow[t]{2}{*}{ Total } & 289 & 427 & 245 & 331 & 334 & 1626 \\
\hline & 17.77 & 26.26 & 15.07 & 20.36 & 20.54 & 100.00 \\
\hline Priors & 0.1845 & 0.26384 & 0.15129 & 0.20357 & 0.1968 & \\
\hline
\end{tabular}




\begin{tabular}{lrrrrrr}
\hline \multicolumn{7}{c}{ Error count estimates for cluster } \\
& 1 & 2 & 3 & 4 & 5 & Total \\
Rate & 0.1600 & 0.0816 & 0.0691 & 0.1360 & 0.0750 & 0.1039 \\
Priors & 0.1845 & 0.2638 & 0.1513 & 0.2036 & 0.1968 & \\
\hline
\end{tabular}


Table 10.13.-Discriminant function analysis of Gloyd's subspecies for males $(\mathrm{n}=866)$.

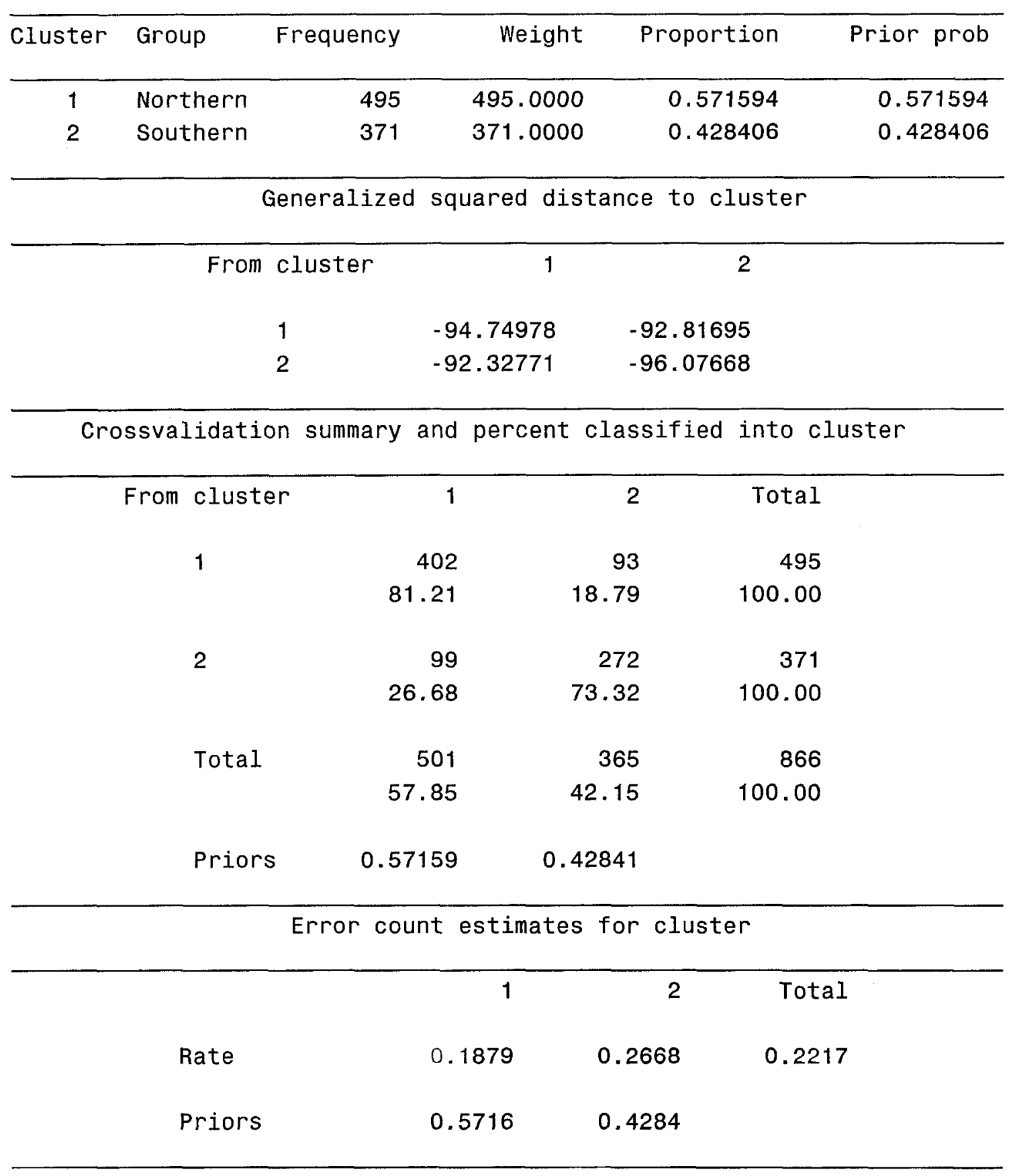


Table 10.14.-Discriminant function analysis of Gloyd's subspecies for females $(n=747)$.

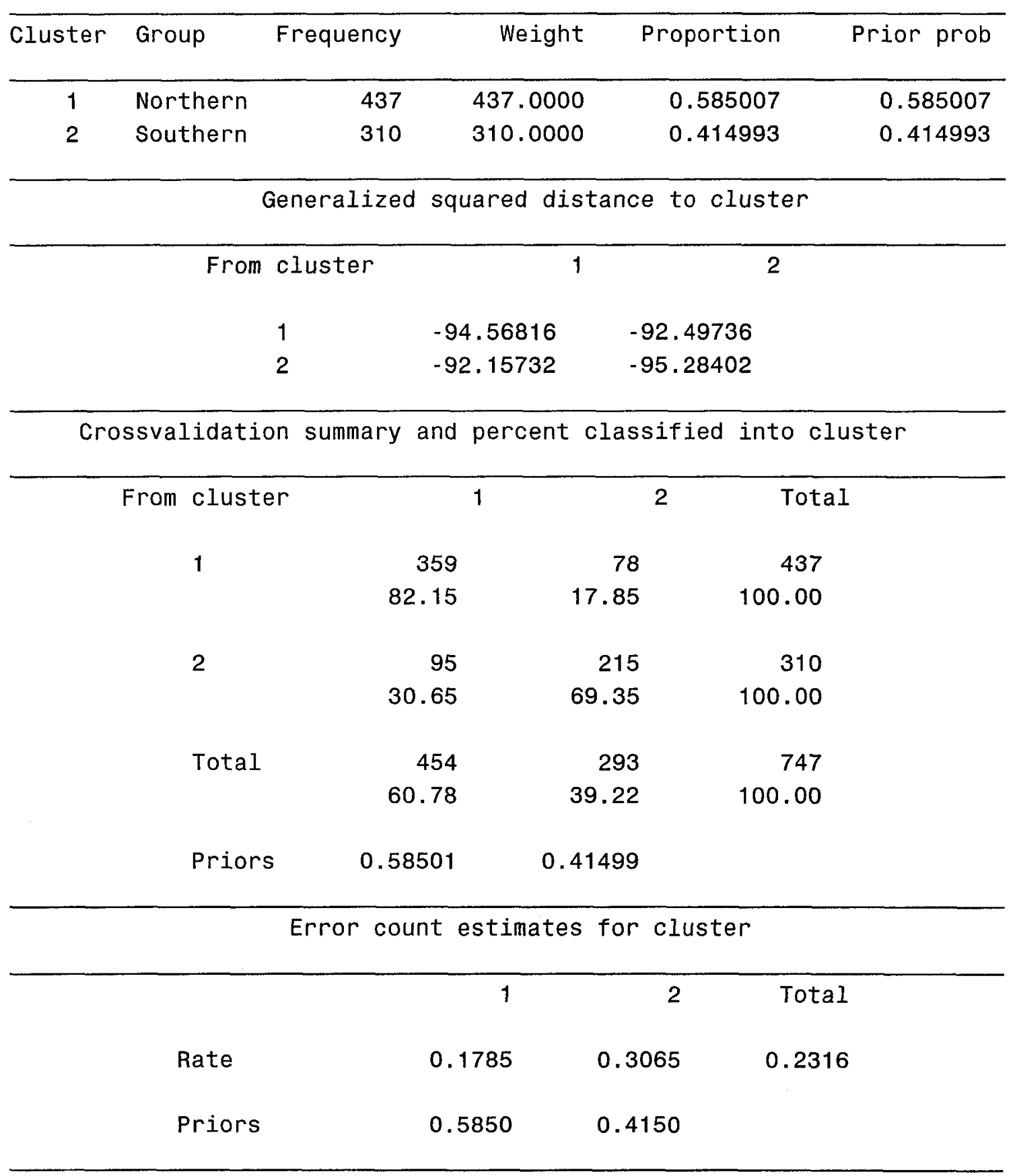


Table 10.15.-Discriminant function analysis of Gloyd's subspecies for combined sexes $(\mathrm{n}=1614)$.

\begin{tabular}{|c|c|c|c|c|c|}
\hline cluster & Group & Frequency & Weight & Proportion & Prior prob \\
\hline 1 & Northern & 933 & 933.0000 & 0.578067 & 0.578067 \\
\hline \multirow[t]{5}{*}{2} & Southern & 681 & 681.0000 & 0.421933 & 0.421933 \\
\hline & \multicolumn{5}{|c|}{ Generalized squared distance to cluster } \\
\hline & \multicolumn{2}{|c|}{ From cluster } & \multicolumn{2}{|r|}{2} & \\
\hline & & 1 & -93.02839 & -91.04317 & \\
\hline & & 2 & -90.77100 & -93.91071 & \\
\hline
\end{tabular}

\begin{tabular}{|c|c|c|c|}
\hline \multicolumn{4}{|c|}{ Crossvalidation summary and percent classified into cluster } \\
\hline From cluster & 1 & 2 & Total \\
\hline \multirow[t]{2}{*}{1} & 754 & 179 & 933 \\
\hline & 80.81 & 19.19 & 100.00 \\
\hline \multirow[t]{2}{*}{2} & 186 & 495 & 681 \\
\hline & 27.31 & 72.69 & 100.00 \\
\hline \multirow[t]{2}{*}{ Total } & 940 & 674 & 1614 \\
\hline & 58.24 & 41.76 & 100.00 \\
\hline Priors & 0.57807 & 0.42193 & \\
\hline \multicolumn{4}{|c|}{ Error count estimates for cluster } \\
\hline & 1 & 2 & Total \\
\hline Rate & 0.1919 & 0.2731 & 0.2261 \\
\hline Priors & 0.5781 & 0.4219 & \\
\hline
\end{tabular}


Table 10.16.-Discriminant function analysis of Martin's regional morphs for males $(\mathrm{n}=867)$.

\begin{tabular}{|c|c|c|c|c|c|}
\hline Cluster & Morph & Frequency & Weight & Proportion & Prior prob \\
\hline 1 & Northern & 253 & 253.0000 & 0.291811 & 0.291811 \\
\hline 2 & Southern & 306 & 306.0000 & 0.352941 & 0.352941 \\
\hline 3 & Western & 308 & 308.0000 & 0.355248 & 0.355248 \\
\hline \multicolumn{6}{|c|}{ Generalized squared distance to cluster } \\
\hline \multicolumn{2}{|r|}{ From cluster } & 1 & 2 & \multicolumn{2}{|c|}{3} \\
\hline \multicolumn{2}{|r|}{1} & -94.60762 & -87.55031 & \multicolumn{2}{|c|}{-90.62171} \\
\hline \multicolumn{2}{|r|}{2} & -87.12345 & .96 .08031 & \multicolumn{2}{|c|}{-92.93480} \\
\hline \multicolumn{2}{|r|}{3} & -90.97430 & -93.36576 & -94.86 & \\
\hline
\end{tabular}

\begin{tabular}{|c|c|c|c|c|c|}
\hline \multicolumn{3}{|c|}{ Crossvalidation summar } & id percent & \multicolumn{2}{|c|}{ issified into cluster } \\
\hline From & cluster & 1 & 2 & 3 & Total \\
\hline \multirow{2}{*}{\multicolumn{2}{|c|}{1}} & 195 & 14 & 44 & 253 \\
\hline & & 77.08 & 5.53 & 17.39 & 100.00 \\
\hline \multirow{2}{*}{\multicolumn{2}{|c|}{2}} & 14 & 219 & 73 & 306 \\
\hline & & 4.58 & 71.57 & 23.86 & 100.00 \\
\hline \multirow{2}{*}{\multicolumn{2}{|c|}{3}} & 51 & 78 & 179 & 308 \\
\hline & & 16.56 & 25.32 & 58.12 & 100.00 \\
\hline \multirow{2}{*}{\multicolumn{2}{|c|}{ Total }} & 260 & 311 & 296 & 867 \\
\hline & & 29.99 & 35.87 & 34.14 & 100.00 \\
\hline \multirow{3}{*}{\multicolumn{2}{|c|}{ Priors }} & 0.29181 & 0.35294 & 0.35525 & \\
\hline & & \multicolumn{3}{|c|}{ Error count estimates for cluster } & \\
\hline & & 1 & 2 & 3 & Total \\
\hline \multicolumn{2}{|r|}{ Rate } & 0.2292 & 0.2843 & 0.4188 & 0.3160 \\
\hline & Priors & 0.2918 & 0.3529 & 0.3552 & \\
\hline
\end{tabular}


Table 10.17.-Discriminant function analysis of Martin's regional morphs for females $(n=748)$.

\begin{tabular}{|c|c|c|c|c|c|}
\hline Cluster & Morph & Frequency & Weight & Proportion & Prior prob \\
\hline 1 & Northern & 198 & 198.0000 & 0.264706 & 0.264706 \\
\hline 2 & Southern & 236 & 236.0000 & 0.315508 & 0.315508 \\
\hline 3 & Western & 314 & 314.0000 & 0.419786 & 0.419786 \\
\hline \multicolumn{6}{|c|}{ Generalized squared distance to cluster } \\
\hline \multicolumn{2}{|r|}{ From cluster } & 1 & 2 & \multicolumn{2}{|c|}{3} \\
\hline \multicolumn{2}{|r|}{1} & -94.02126 & -87.29689 & \multicolumn{2}{|c|}{-90.75693} \\
\hline \multicolumn{2}{|r|}{2} & -86.98267 & -95.26956 & \multicolumn{2}{|c|}{-93.13080} \\
\hline \multicolumn{2}{|r|}{3} & -89.81381 & -92.89618 & -94.89 & \\
\hline
\end{tabular}

Crossvalidation summary and percent classified into cluster

\begin{tabular}{|c|c|c|c|c|c|}
\hline Fron & n cluster & 1 & 2 & 3 & Total \\
\hline \multirow{2}{*}{\multicolumn{2}{|c|}{1}} & 142 & 11 & 45 & 198 \\
\hline & & 71.72 & 5.56 & 22.73 & 100.00 \\
\hline \multirow{2}{*}{\multicolumn{2}{|c|}{2}} & 10 & 154 & 72 & 236 \\
\hline & & 4.24 & 65.25 & 30.51 & 100.00 \\
\hline \multirow{2}{*}{\multicolumn{2}{|c|}{3}} & 33 & 73 & 208 & 314 \\
\hline & & 10.51 & 23.25 & 66.24 & 100.00 \\
\hline \multirow{2}{*}{\multicolumn{2}{|c|}{ Total }} & 185 & 238 & 325 & 748 \\
\hline & & 24.73 & 31.82 & 43.45 & 100.00 \\
\hline \multirow{3}{*}{\multicolumn{2}{|c|}{ Priors }} & 0.26471 & 0.31551 & 0.41979 & \\
\hline & & \multicolumn{3}{|c|}{ Error count estimates for cluster } & \\
\hline & & \multicolumn{2}{|c|}{1} & 3 & Total \\
\hline & Rate & 0.282 & 0.3475 & 0.3376 & 0.3262 \\
\hline & Priors & 0.2647 & 0.3155 & 0.4198 & \\
\hline
\end{tabular}


Table 10.18.-Discriminant function analysis of Martin's regional morphs for combined sexes $(n=1616)$.

\begin{tabular}{clrrrr}
\hline Cluster & Morph & Frequency & Weight & Proportion & Prior prob \\
\hline 1 & Northern & 452 & 452.0000 & 0.279703 & 0.279703 \\
2 & Southern & 542 & 542.0000 & 0.335396 & 0.335396 \\
3 & Western & 622 & 622.0000 & 0.384901 & 0.384901
\end{tabular}

\begin{tabular}{|c|c|c|c|c|}
\hline \multicolumn{5}{|c|}{ Generalized squared distance to cluster } \\
\hline From cluster & 1 & 2 & \multicolumn{2}{|r|}{3} \\
\hline 1 & -92.37633 & -86.18628 & \multicolumn{2}{|c|}{-89.47057} \\
\hline 2 & -86.04055 & -93.83964 & \multicolumn{2}{|c|}{-91.40816} \\
\hline 3 & -89.34862 & -91.42401 & \multicolumn{2}{|c|}{-93.12702} \\
\hline \multicolumn{5}{|c|}{ Crossvalidation summary and percent classified into cluster } \\
\hline From cluster & 1 & 2 & 3 & Total \\
\hline \multirow[t]{2}{*}{1} & 318 & 30 & 104 & 452 \\
\hline & 70.35 & 6.64 & 23.01 & 100.00 \\
\hline \multirow[t]{2}{*}{2} & 24 & 398 & 120 & 542 \\
\hline & 4.43 & 73.43 & 22.14 & 100.00 \\
\hline \multirow[t]{2}{*}{3} & 75 & 146 & 401 & 622 \\
\hline & 12.06 & 23.47 & 64.47 & 100.00 \\
\hline \multirow[t]{2}{*}{ Total } & 417 & 574 & 625 & 1616 \\
\hline & 25.80 & 35.52 & 38.68 & 100.00 \\
\hline Priors & 0.2797 & 0.3354 & 0.3849 & \\
\hline \multicolumn{5}{|c|}{ Error count estimates for cluster } \\
\hline & 1 & 2 & 3 & Total \\
\hline Rate & 0.2965 & 0.2657 & 0.3553 & 0.3088 \\
\hline Priors & 0.2797 & 0.3354 & 0.3849 & \\
\hline
\end{tabular}




\section{APPENDIX XI}

\section{UNIVARIATE STATISTICS OF MORPHOMETRIC DATA FOR MALES}

Mean values, descriptive statistics, and ANOVAs of 7 morphometric characters in 12 study areas for males. Character abbreviations are defined in Appendix II. Study areas are displayed in Figure 5. For each study area, the following data are reported for each character: the $F$-ratio $(F)$, level of significance $(P)$, sample size for that character $(n)$, minimum value (Min), maximum value (Max), mean value (mean), and standard deviation (SD).

\begin{tabular}{|c|c|c|c|c|c|c|c|c|}
\hline Region & $\begin{array}{l}\text { Statistic } \\
F \\
P\end{array}$ & $\begin{array}{r}\text { SVL } \\
13.00 \\
0.0001\end{array}$ & $\begin{array}{r}\mathrm{TL} \\
21.96 \\
0.0001\end{array}$ & $\begin{array}{r}\text { HLM } \\
15.90 \\
0.0001\end{array}$ & $\begin{array}{r}\text { HLS } \\
11.77 \\
0.0001\end{array}$ & $\begin{array}{r}\mathrm{HW} \\
7.23 \\
0.0001\end{array}$ & $\begin{array}{r}\mathrm{QL} \\
12.68 \\
0.0001\end{array}$ & $\begin{array}{r}\mathrm{ML} \\
16.00 \\
0.0001\end{array}$ \\
\hline FL & $\begin{array}{l}n \\
\text { Min } \\
\text { Max } \\
\text { Mean } \\
\text { SD }\end{array}$ & $\begin{array}{r}30 \\
865 \\
1380 \\
1126.8 \\
129.10\end{array}$ & $\begin{array}{r}29 \\
73 \\
116 \\
99.2 \\
11.08\end{array}$ & $\begin{array}{r}25 \\
40.5 \\
54.5 \\
47.48 \\
4.158\end{array}$ & $\begin{array}{r}24 \\
27.3 \\
36.9 \\
31.59 \\
2.576\end{array}$ & $\begin{array}{r}20 \\
30.1 \\
44.3 \\
37.10 \\
4.194\end{array}$ & $\begin{array}{r}22 \\
15.0 \\
22.9 \\
19.02 \\
2.264\end{array}$ & $\begin{array}{r}23 \\
37.9 \\
51.6 \\
45.24 \\
3.723\end{array}$ \\
\hline ILS & $\begin{array}{l}n \\
\text { Min } \\
\text { Max } \\
\text { Mean } \\
\text { SD }\end{array}$ & $\begin{array}{r}16 \\
828 \\
1263 \\
1006.7 \\
131.20\end{array}$ & $\begin{array}{r}15 \\
65 \\
102 \\
82.9 \\
9.27\end{array}$ & $\begin{array}{r}16 \\
36.8 \\
50.8 \\
42.86 \\
4.278\end{array}$ & $\begin{array}{r}15 \\
25.9 \\
34.2 \\
29.06 \\
2.374\end{array}$ & $\begin{array}{r}5 \\
27.3 \\
35.9 \\
31.76 \\
3.711\end{array}$ & $\begin{array}{r}15 \\
14.1 \\
21.1 \\
16.95 \\
2.161\end{array}$ & $\begin{array}{r}14 \\
34.7 \\
47.8 \\
40.92 \\
3.995\end{array}$ \\
\hline $\mathrm{KS}$ & $\begin{array}{l}n \\
\text { Min } \\
\text { Max } \\
\text { Mean } \\
\text { SD }\end{array}$ & $\begin{array}{r}21 \\
810 \\
1215 \\
947.1 \\
123.10\end{array}$ & $\begin{array}{r}21 \\
65 \\
91 \\
77.3 \\
7.00\end{array}$ & $\begin{array}{r}20 \\
36.8 \\
50.0 \\
41.51 \\
3.872\end{array}$ & $\begin{array}{r}19 \\
25.2 \\
34.2 \\
28.44 \\
2.398\end{array}$ & $\begin{array}{r}12 \\
27.0 \\
37.8 \\
31.68 \\
3.642\end{array}$ & $\begin{array}{r}20 \\
14.0 \\
20.4 \\
16.43 \\
1.855\end{array}$ & $\begin{array}{r}18 \\
34.4 \\
46.8 \\
38.91 \\
3.832\end{array}$ \\
\hline LAS & $\begin{array}{l}n \\
\text { Min } \\
\text { Max } \\
\text { Mean } \\
\text { SD }\end{array}$ & $\begin{array}{r}21 \\
810 \\
1670 \\
1186.7 \\
214.51\end{array}$ & $\begin{array}{r}20 \\
71 \\
138 \\
101.3 \\
18.11\end{array}$ & $\begin{array}{r}20 \\
37.7 \\
60.5 \\
49.58 \\
6.659\end{array}$ & $\begin{array}{r}19 \\
26.1 \\
39.5 \\
32.56 \\
3.844\end{array}$ & $\begin{array}{r}9 \\
27.5 \\
46.5 \\
36.53 \\
5.948\end{array}$ & $\begin{array}{r}18 \\
14.0 \\
25.6 \\
20.09 \\
3.083\end{array}$ & $\begin{array}{r}20 \\
34.2 \\
57.8 \\
47.01 \\
6.505\end{array}$ \\
\hline MNWI & $\begin{array}{l}n \\
\text { Min } \\
\text { Max } \\
\text { Mean } \\
\text { SD }\end{array}$ & $\begin{array}{r}17 \\
830 \\
1127 \\
963.5 \\
78.20\end{array}$ & $\begin{array}{r}17 \\
72 \\
88 \\
80.8 \\
5.20\end{array}$ & $\begin{array}{r}14 \\
38.5 \\
46.6 \\
41.14 \\
2.452\end{array}$ & $\begin{array}{r}15 \\
25.1 \\
33.4 \\
28.23 \\
2.001\end{array}$ & $\begin{array}{r}12 \\
27.4 \\
35.3 \\
30.73 \\
2.511\end{array}$ & $\begin{array}{r}16 \\
15.4 \\
19.8 \\
16.92 \\
1.262\end{array}$ & $\begin{array}{r}13 \\
35.3 \\
44.5 \\
38.63 \\
2.594\end{array}$ \\
\hline MO & $\begin{array}{l}n \\
\text { Min } \\
\text { Max } \\
\text { Mean } \\
\text { SD }\end{array}$ & $\begin{array}{r}24 \\
802 \\
1210 \\
982.2 \\
107.29\end{array}$ & $\begin{array}{r}23 \\
65 \\
100 \\
80.3 \\
8.70\end{array}$ & $\begin{array}{r}24 \\
37.5 \\
49.1 \\
42.79 \\
3.306\end{array}$ & $\begin{array}{r}23 \\
25.9 \\
33.0 \\
29.15 \\
2.023\end{array}$ & $\begin{array}{r}16 \\
28.5 \\
35.6 \\
32.16 \\
1.947\end{array}$ & $\begin{array}{r}23 \\
14.1 \\
20.9 \\
16.96 \\
1.799\end{array}$ & $\begin{array}{r}23 \\
35.6 \\
45.6 \\
39.73 \\
2.891\end{array}$ \\
\hline
\end{tabular}




\begin{tabular}{|c|c|c|c|c|c|c|c|c|}
\hline Region & Statistic & SVL & TL & HLM & HLS & $\mathrm{HW}$ & $\overline{Q L}$ & ML \\
\hline \multirow[t]{5}{*}{ NCCP } & $n$ & 37 & 35 & 32 & 31 & 13 & 32 & 30 \\
\hline & Min & 842 & 72 & 40.6 & 26.4 & 29.5 & 15.6 & 39.1 \\
\hline & Max & 1485 & 116 & 57.4 & 39.4 & 44.5 & 23.8 & 53.7 \\
\hline & Mean & 1127.6 & 95.6 & 48.65 & 32.22 & 36.68 & 19.84 & 46.15 \\
\hline & $\mathrm{SD}$ & 150.63 & 10.56 & 4.740 & 2.989 & 4.873 & 2.146 & 4.441 \\
\hline \multirow[t]{5}{*}{ NCMT } & $n$ & 29 & 29 & 28 & 27 & 16 & 28 & 27 \\
\hline & Min & 822 & 65 & 38.2 & 24.6 & 28.6 & 14.1 & 36.0 \\
\hline & Max & 1150 & 97 & 55.0 & 35.5 & 36.5 & 22.7 & 51.5 \\
\hline & Mean & 980.1 & 79.2 & 44.33 & 29.86 & 33.53 & 17.71 & 41.46 \\
\hline & SD & 107.89 & 8.02 & 3.798 & 2.269 & 2.722 & 2.054 & 3.590 \\
\hline \multirow[t]{5}{*}{ NCPD } & $n$ & 9 & 9 & 7 & 7 & 5 & 8 & 8 \\
\hline & Min & 858 & 68 & 40.1 & 28.0 & 30.6 & 15.6 & 38.0 \\
\hline & $\operatorname{Max}$ & 1304 & 104 & 54.2 & 35.5 & 41.8 & 22.0 & 50.4 \\
\hline & Mean & 1117.8 & 89.3 & 48.09 & 32.19 & 36.20 & 19.49 & 45.40 \\
\hline & SD & 155.78 & 12.69 & 5.604 & 3.030 & 5.201 & 2.297 & 4.975 \\
\hline \multirow[t]{5}{*}{ NYN } & $n$ & 11 & 11 & 10 & 10 & 2 & 10 & 10 \\
\hline & Min & 805 & 68 & 36.7 & 25.4 & 33.2 & 13.5 & 34.5 \\
\hline & $\operatorname{Max}$ & 1168 & 91 & 46.2 & 31.1 & 33.3 & 18.5 & 43.1 \\
\hline & Mean & 953.0 & 81.4 & 41.49 & 28.45 & 33.25 & 16.25 & 38.65 \\
\hline & $\mathrm{SD}$ & 107.53 & 7.75 & 2.876 & 1.885 & 0.071 & 1.683 & 2.888 \\
\hline \multirow[t]{5}{*}{ PAE } & $n$ & 21 & 21 & 21 & 21 & 16 & 21 & 19 \\
\hline & Min & 800 & 69 & 35.7 & 26.0 & 25.5 & 14.0 & 34.4 \\
\hline & $\operatorname{Max}$ & 1112 & 85 & 47.5 & 32.1 & 37.1 & 18.5 & 44.1 \\
\hline & Mean & 975.7 & 78.8 & 40.62 & 28.29 & 31.34 & 16.24 & 38.58 \\
\hline & SD & 97.55 & 3.75 & 3.305 & 1.529 & 3.235 & 1.259 & 2.709 \\
\hline \multirow{5}{*}{ VAMT } & $n$ & 52 & 50 & 49 & 49 & 38 & 46 & 43 \\
\hline & Min & 800 & 63 & 34.6 & 24.8 & 26.2 & 13.2 & 32.8 \\
\hline & $\operatorname{Max}$ & 1100 & 93 & 46.5 & 32.5 & 37.3 & 19.4 & 44.9 \\
\hline & Mean & 925.2 & 78.2 & 40.88 & 27.89 & 30.82 & 16.26 & 38.69 \\
\hline & SD & 79.95 & 6.72 & 2.790 & 2.038 & 2.919 & 1.504 & 2.885 \\
\hline
\end{tabular}

Reproduced with permission of the copyright owner. Further reproduction prohibited without permission. 


\section{APPENDIX XII}

\section{UNIVARIATE STATISTICS OF MORPHOMETRIC DATA FOR FEMALES}

Mean values, descriptive statistics, and ANOVAs of 7 morphometric characters in 12 study areas for females. Character abbreviations are defined in Appendix II. Study areas are displayed in Figure 5. For each study area, the following data are reported for each character: the $F$-ratio $(F)$, level of significance $(P)$, sample size for that character $(n)$, minimum value (Min), maximum value (Max), mean value (mean), and standard deviation (SD).

\begin{tabular}{|c|c|c|c|c|c|c|c|c|}
\hline Region & $\begin{array}{l}\text { Statistic } \\
F \\
P\end{array}$ & $\begin{array}{r}\text { SVL } \\
24.43 \\
0.0001\end{array}$ & $\begin{array}{r}\mathrm{TL} \\
19.39 \\
0.0001\end{array}$ & $\begin{array}{r}\text { HLM } \\
24.55 \\
0.0001\end{array}$ & $\begin{array}{r}\text { HLS } \\
16.95 \\
0.0001\end{array}$ & $\begin{array}{r}\text { HW } \\
9.02 \\
0.0001\end{array}$ & $\begin{array}{r}\mathrm{QL} \\
23.21 \\
0.0001\end{array}$ & $\begin{array}{r}\mathrm{ML} \\
26.99 \\
0.0001\end{array}$ \\
\hline FL & $\begin{array}{l}n \\
\text { Min } \\
\text { Max } \\
\text { Mean } \\
\text { SD }\end{array}$ & $\begin{array}{r}13 \\
801 \\
1236 \\
1085.9 \\
116.77\end{array}$ & $\begin{array}{r}12 \\
58 \\
77 \\
71.3 \\
6.68\end{array}$ & $\begin{array}{r}13 \\
37.7 \\
49.7 \\
45.90 \\
3.840\end{array}$ & $\begin{array}{r}11 \\
24.0 \\
34.0 \\
30.25 \\
2.893\end{array}$ & $\begin{array}{r}10 \\
23.6 \\
39.9 \\
33.46 \\
4.361\end{array}$ & $\begin{array}{r}9 \\
15.0 \\
21.6 \\
18.86 \\
2.288\end{array}$ & $\begin{array}{r}13 \\
35.5 \\
49.5 \\
44.42 \\
3.835\end{array}$ \\
\hline ILS & $\begin{array}{l}n \\
\text { Min } \\
\text { Max } \\
\text { Mean } \\
\text { SD }\end{array}$ & $\begin{array}{r}16 \\
840 \\
1020 \\
928.1 \\
58.24\end{array}$ & $\begin{array}{r}13 \\
53 \\
67 \\
60.8 \\
4.86\end{array}$ & $\begin{array}{r}13 \\
37.6 \\
43.6 \\
40.63 \\
2.058\end{array}$ & $\begin{array}{r}13 \\
26.1 \\
30.3 \\
28.56 \\
1.113\end{array}$ & $\begin{array}{r}6 \\
28.1 \\
30.8 \\
30.00 \\
1.018\end{array}$ & $\begin{array}{r}14 \\
14.6 \\
17.7 \\
15.80 \\
0.962\end{array}$ & $\begin{array}{r}13 \\
34.4 \\
42.5 \\
37.86 \\
2.138\end{array}$ \\
\hline KS & $\begin{array}{l}n \\
\text { Min } \\
\text { Max } \\
\text { Mean } \\
\text { SD }\end{array}$ & $\begin{array}{r}27 \\
810 \\
977 \\
879.7 \\
47.07\end{array}$ & $\begin{array}{r}27 \\
48 \\
67 \\
56.5 \\
4.37\end{array}$ & $\begin{array}{r}27 \\
35.8 \\
43.9 \\
39.34 \\
2.056\end{array}$ & $\begin{array}{r}27 \\
24.6 \\
31.0 \\
26.93 \\
1.628\end{array}$ & $\begin{array}{r}20 \\
26.0 \\
34.3 \\
29.32 \\
2.268\end{array}$ & $\begin{array}{r}27 \\
13.6 \\
17.5 \\
15.22 \\
0.946\end{array}$ & $\begin{array}{r}26 \\
32.9 \\
41.4 \\
36.89 \\
1.820\end{array}$ \\
\hline LAS & $\begin{array}{l}n \\
\text { Min } \\
\text { Max } \\
\text { Mean } \\
\text { SD }\end{array}$ & $\begin{array}{r}7 \\
848 \\
1344 \\
1055.3 \\
217.17\end{array}$ & $\begin{array}{r}7 \\
56 \\
85 \\
70.1 \\
11.45\end{array}$ & $\begin{array}{r}7 \\
37.9 \\
55.5 \\
45.91 \\
7.282\end{array}$ & $\begin{array}{r}7 \\
25.9 \\
37.4 \\
30.54 \\
4.192\end{array}$ & $\begin{array}{r}4 \\
29.5 \\
42.7 \\
36.28 \\
5.940\end{array}$ & $\begin{array}{r}7 \\
14.8 \\
23.2 \\
18.46 \\
3.158\end{array}$ & $\begin{array}{r}7 \\
35.6 \\
51.2 \\
42.56 \\
6.512\end{array}$ \\
\hline MNWI & $\begin{array}{l}n \\
\text { Min } \\
\text { Max } \\
\text { Mean } \\
\text { SD }\end{array}$ & $\begin{array}{r}19 \\
817 \\
1107 \\
909.6 \\
68.19\end{array}$ & $\begin{array}{r}19 \\
53 \\
76 \\
59.7 \\
5.58\end{array}$ & $\begin{array}{r}19 \\
36.5 \\
48.3 \\
40.10 \\
2.907\end{array}$ & $\begin{array}{r}19 \\
24.3 \\
31.7 \\
26.66 \\
1.945\end{array}$ & $\begin{array}{r}11 \\
27.0 \\
34.2 \\
29.83 \\
2.291\end{array}$ & $\begin{array}{r}19 \\
14.5 \\
19.3 \\
16.18 \\
1.206\end{array}$ & $\begin{array}{r}19 \\
34.3 \\
44.5 \\
37.26 \\
2.825\end{array}$ \\
\hline MO & $\begin{array}{l}n \\
\text { Min } \\
\text { Max } \\
\text { Mean } \\
\text { SD }\end{array}$ & $\begin{array}{r}21 \\
803 \\
992 \\
870.8 \\
47.34\end{array}$ & $\begin{array}{r}21 \\
51 \\
68 \\
57.6 \\
4.68\end{array}$ & $\begin{array}{r}21 \\
35.5 \\
43.4 \\
39.18 \\
2.317\end{array}$ & $\begin{array}{r}21 \\
24.2 \\
29.7 \\
27.04 \\
1.569\end{array}$ & $\begin{array}{r}11 \\
26.6 \\
31.9 \\
28.65 \\
1.618\end{array}$ & $\begin{array}{r}21 \\
14.0 \\
17.1 \\
15.32 \\
0.945\end{array}$ & $\begin{array}{r}21 \\
33.2 \\
40.0 \\
36.20 \\
2.293\end{array}$ \\
\hline
\end{tabular}




\begin{tabular}{|c|c|c|c|c|c|c|c|c|}
\hline Region & Statistic & SVL & TL & HLM & HLS & $\mathrm{HW}$ & $\mathrm{QL}$ & ML \\
\hline \multirow[t]{5}{*}{$\mathrm{NCCP}$} & $n$ & 32 & 31 & 30 & 30 & 16 & 31 & 29 \\
\hline & Min & 830 & 56 & 38.9 & 24.3 & 30.0 & 14.6 & 37.0 \\
\hline & Max & 1304 & 82 & 58.1 & 37.8 & 45.0 & 24.3 & 56.2 \\
\hline & Mean & 1115.9 & 71.4 & 48.22 & 31.93 & 36.18 & 19.69 & 46.19 \\
\hline & SD & 114.00 & 7.27 & 4.578 & 2.776 & 3.884 & 2.150 & 4.478 \\
\hline \multirow[t]{5}{*}{ NCMT } & $n$ & 16 & 16 & 15 & 15 & 8 & 15 & 14 \\
\hline & Min & 805 & 45 & 35.7 & 25.2 & 25.5 & 14.3 & 33.0 \\
\hline & Max & 945 & 61 & 44.1 & 31.3 & 33.9 & 17.4 & 41.2 \\
\hline & Mean & 882.5 & 54.8 & 40.79 & 27.65 & 30.16 & 16.38 & 38.43 \\
\hline & SD & 40.24 & 4.39 & 2.182 & 1.747 & 2.934 & 0.978 & 2.274 \\
\hline \multirow[t]{5}{*}{ NCPD } & $n$ & 14 & 14 & 13 & 12 & 7 & 11 & 12 \\
\hline & Min & 833 & 55 & 40.0 & 26.8 & 28.3 & 15.8 & 37.7 \\
\hline & Max & 1297 & 90 & 53.6 & 35.2 & 40.7 & 22.5 & 52.1 \\
\hline & Mean & 1036.9 & 66.9 & 47.22 & 31.07 & 34.24 & 18.94 & 44.50 \\
\hline & SD & 126.56 & 9.59 & 3.511 & 2.070 & 3.734 & 1.923 & 3.701 \\
\hline \multirow[t]{5}{*}{ NYN } & $n$ & 8 & 7 & 8 & 8 & 4 & 8 & 7 \\
\hline & Min & 823 & 57 & 36.2 & 24.3 & 27.2 & 13.2 & 33.6 \\
\hline & $\operatorname{Max}$ & 1080 & 67 & 44.6 & 32.3 & 30.8 & 18.6 & 42.6 \\
\hline & Mean & 944.4 & 62.6 & 40.58 & 27.76 & 28.73 & 15.90 & 38.46 \\
\hline & SD & 95.80 & 4.24 & 3.231 & 2.573 & 1.595 & 1.910 & 3.008 \\
\hline \multirow[t]{5}{*}{ PAE } & $n$ & 23 & 23 & 23 & 23 & 17 & 23 & 23 \\
\hline & Min & 822 & 50 & 35.4 & 24.2 & 25.2 & 13.9 & 33.3 \\
\hline & $\operatorname{Max}$ & 1020 & 70 & 45.4 & 30.3 & 38.7 & 18.2 & 41.3 \\
\hline & Mean & 902.0 & 59.4 & 39.19 & 27.10 & 29.88 & 15.56 & 37.14 \\
\hline & SD & 48.03 & 4.60 & 2.641 & 1.624 & 3.088 & 1.215 & 2.039 \\
\hline \multirow[t]{5}{*}{ VAMT } & $n$ & 26 & 24 & 22 & 19 & 16 & 22 & 20 \\
\hline & Min & 810 & 51 & 33.8 & 23.5 & 25.2 & 12.8 & 33.3 \\
\hline & $\operatorname{Max}$ & 961 & 68 & 43.6 & 30.0 & 34.0 & 17.3 & 41.2 \\
\hline & Mean & 869.4 & 56.9 & 38.20 & 26.22 & 29.44 & 15.25 & 36.77 \\
\hline & SD & 46.31 & 4.23 & 2.222 & 1.584 & 2.282 & 1.082 & 2.005 \\
\hline
\end{tabular}




\section{APPENDIX XIII}

\section{FACTOR ANALYSIS OF MORPHOMETRIC DATA}

Factor analysis of six characters of the morphometric data $(n=1887)$. Eigenvalues of the correlation matrix are listed. The initial factor method was principal components. The factor pattern was computed for four factors. The variances explained by each factor were: Factor 1 (5.8115484), Factor 2 (0.1313551), Factor 3 (0.0235682), and Factor 4 (0.0170393).

\begin{tabular}{ccccc}
\hline Factor & Eigenvalue & Difference & Proportion & Cumulative \\
\hline 1 & 5.81154842 & 5.68019331 & 0.9686 & 0.9686 \\
2 & 0.13135511 & 0.10778687 & 0.0219 & 0.9905 \\
3 & 0.02356824 & 0.00652890 & 0.0039 & 0.9944 \\
4 & 0.01703934 & 0.00574397 & 0.0028 & 0.9973 \\
5 & 0.01129538 & 0.00610186 & 0.0019 & 0.9991 \\
6 & 0.00519351 & & 0.0009 & 1.0000
\end{tabular}

Factor pattern

\begin{tabular}{lrrrr}
\hline Character & Factor 1 & Factor 2 & Factor 3 & Factor 4 \\
\hline SVL & 0.99212 & -0.03841 & -0.03063 & -0.11521 \\
TL & 0.94307 & 0.33245 & -0.00049 & 0.00957 \\
HLM & 0.99445 & -0.06868 & -0.00264 & 0.02636 \\
HLS & 0.98905 & -0.06469 & 0.12824 & 0.00432 \\
QL & 0.99067 & -0.07738 & -0.07661 & 0.04548 \\
ML & 0.99462 & -0.06682 & -0.01756 & 0.02989
\end{tabular}

Standardized scoring coefficients

\begin{tabular}{crrrr}
\hline Character & Factor 1 & Factor 2 & Factor 3 & Factor 4 \\
\hline SVL & 0.17071562 & -0.2924477 & -1.2997552 & -6.7611558 \\
TL & 0.16227536 & 2.5309288 & -0.0208264 & 0.5615962 \\
HLM & 0.17111694 & -0.5228796 & -0.1120831 & 1.5472337 \\
HLS & 0.17018702 & -0.4925075 & 5.4412808 & 0.2535769 \\
QL & 0.17046521 & -0.5891133 & -3.2503848 & 2.6691312 \\
ML & 0.17114627 & -0.5087271 & -0.7450377 & 1.7540215
\end{tabular}




\title{
VITA
}

\author{
John R. Allsteadt \\ Department of Biological Sciences \\ Old Dominion University \\ Norfolk, Virginia 23529
}

\section{Education}

Doctor of Philosophy, Ecological Sciences, Old Dominion University, Norfolk, Virginia, August 2003

Master of Science, Biology, University of North Dakota, Grand Forks, North Dakota, August 1993

Bachelor of Arts, Biology, Lawrence University, Appleton, Wisconsin, June 1987

\section{Research Advisors and Projects}

\section{Doctor of Philosophy}

Advisor: Dr. Alan H. Savitzky, Department of Biological Sciences, Old Dominion University, Norfolk, Virginia

Project: Geographic Variation in the Morphology of Crotalus horridus (Serpentes: Viperidae)

\section{Master of Science}

Advisor: Dr. Jeffrey W. Lang, Department of Biology, University of North Dakota, Grand Forks, North Dakota

Project: Incubation Temperature Affects Body Size, Energy Reserves, and Sex of Hatchling Alligator mississippiensis

\section{Bachelor of Arts}

Academic Advisor: Dr. Bradford Rence, Biology Department, Lawrence University, Appleton, Wisconsin

Project Advisor: Dr. Christopher Vaughan, Programa Regional en Manejo de Vida Silvestre de la Universidad Nacional, Heredia, Costa Rica

Project: The Ecology of Caiman crocodilus in Caño Negro National Wildlife Refuge, Costa Rica 